San Jose State University

SJSU ScholarWorks

Master's Theses

Master's Theses and Graduate Research

Fall 2019

\title{
Synthesis and Study of Verdazyl Stable Free Radical Substituted Oligothiophenes for Magnetoresistive and Spintronic Properties
}

Amir Shakouri Mansouri

San Jose State University

Follow this and additional works at: https://scholarworks.sjsu.edu/etd_theses

\section{Recommended Citation}

Mansouri, Amir Shakouri, "Synthesis and Study of Verdazyl Stable Free Radical Substituted

Oligothiophenes for Magnetoresistive and Spintronic Properties" (2019). Master's Theses. 5070.

DOI: https://doi.org/10.31979/etd.4see-jncj

https://scholarworks.sjsu.edu/etd_theses/5070

This Thesis is brought to you for free and open access by the Master's Theses and Graduate Research at SJSU

ScholarWorks. It has been accepted for inclusion in Master's Theses by an authorized administrator of SJSU

ScholarWorks. For more information, please contact scholarworks@sjsu.edu. 
SYNTHESIS AND STUDY OF VERDAZYL STABLE FREE RADICAL SUBSTITUTED OLIGOTHIOPHENES FOR MAGNETORESISTIVE AND SPINTRONIC PROPERTIES

\author{
A Thesis \\ Presented to \\ The Faculty of the Department of Chemistry \\ San José State University \\ In Partial Fulfillment \\ of the Requirements for the Degree \\ Master of Science
}

by

Amir Mansouri

December 2019 
(C) 2019

Amir Mansouri

ALL RIGHTS RESERVED 
The Designated Thesis Committee Approves the Thesis Titled

SYNTHESIS AND STUDY OF VERDAZYL STABLE FREE RADICAL SUBSTITUTED OLIGOTHIOPHENES FOR MAGNETORESISTIVE AND SPINTRONIC PROPERTIES

By

Amir Mansouri

APPROVED FOR THE DEPARTMENT OF CHEMISTRY

SAN JOSE STATE UNIVERSITY

OCTOBER 2019

David Brook. Ph.D. Department of Chemistry

Roy Okuda. Ph.D. Department of Chemistry

Roger Terrill. Ph.D. Department of Chemistry 


\section{ABSTRACT \\ SYNTHESIS AND STUDY OF VERDAZYL STABLE FREE RADICAL SUBSTITUTED OLIGOTHIOPHENES FOR MAGNETORESISTIVE AND SPINTRONIC PROPERTIES}

\section{By Amir Mansouri}

Interest in spin polarizing materials and their application in electronic devices in the field of spintronics has been steadily growing in recent years. The development of wholly organic spin polarizing materials has been of particular interest due to the material flexibility, solution processing, synthetic modifiability, and longer spin lifetimes of organics compared to inorganic analogs. One class of organic compounds with significant room for exploration is polyconjugated $\pi$ systems coupled with stable radicals. We have explored the synthesis of oligothiophenes substituted with verdazyl stable free radicals with differing thiophene chain lengths, through a series of halogenation, borylation, and palladium catalyzed Suzuki-Miyarua coupling reactions. The characterization methods employed include IR, NMR, UV-Vis, ESR, GC/LC-MS, and X-ray crystallography. We have successfully synthesized two novel bisverdazyls with conjugated oligomer spacers and analyzed the magnetism, electrochemical redox potentials, crystal structure, and molecular packing of the aforementioned diradicals. We hope to build on this work by analyzing the bisverdazyls for their magnetoresistance, conductivity, and spin polarization properties. Furthermore, we also plan to synthesize more efficient bisverdazyl conjugated spacers utilizing EDOT units, which promise improved conductivity and spin propagation. 


\section{ACKNOWLEDGEMENTS}

I would like to take this opportunity to thank my research advisor Dr. David Brook for his continuous support and guidance through my graduate research. I attribute Dr. Brook's extensive knowledge in the field of chemistry, and his creative approach to problem solving, along with patient discussion of research findings, for my fruitful time at San Jose State University. My appreciation also extends to my thesis committee members Dr. Roger Terrill and Dr. Roy Okuda for their time and academic mentorship, which has greatly enriched my learning experience. I am also very grateful for the assistance provided by other members of the Brook research lab, Jeffery DaRos, Alejandro Herrera, Alisa Clemens, and Samira Vakilzadeh, to name a few.

I cannot overstate the significance of the support I received from my father, brothers and friends throughout my graduate program. The encouragement they provided helped me work though some of the difficulties and setbacks I faced through my time in academia. 


\section{TABLE OF CONTENTS}

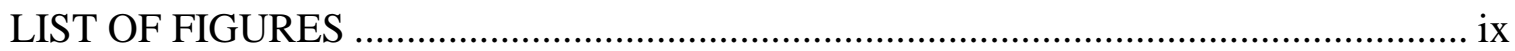

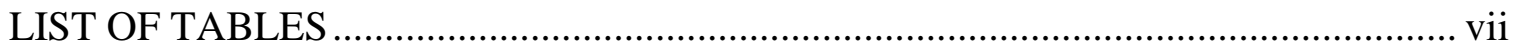

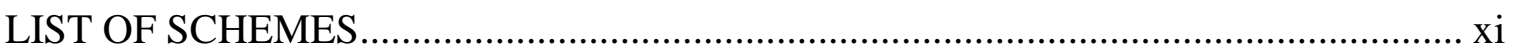

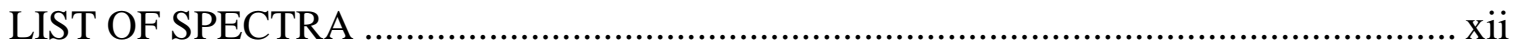

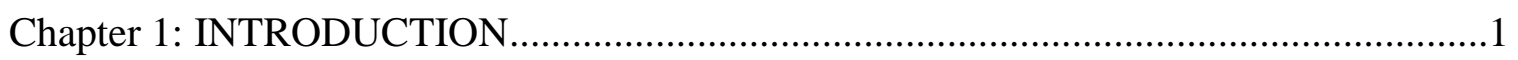

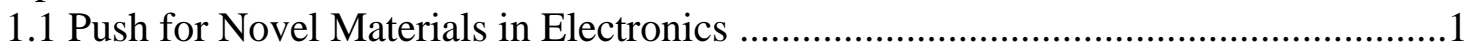

1.2 Historical Overview of Electronic Components ……................................................3

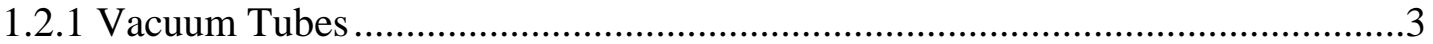

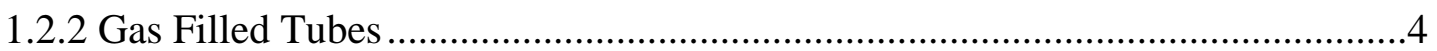

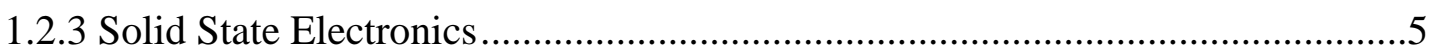

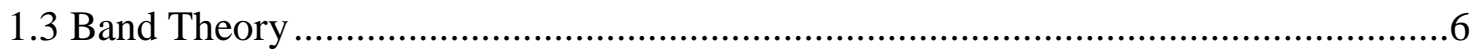

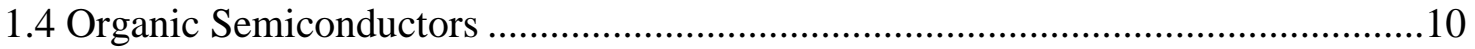

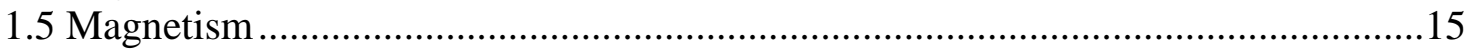

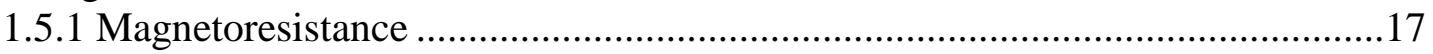

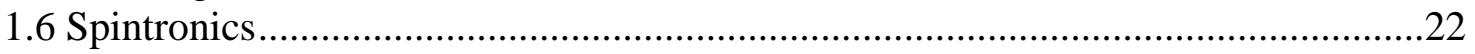

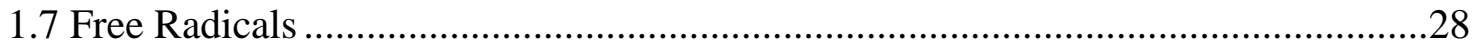

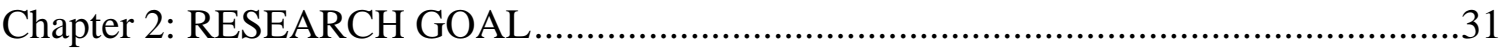

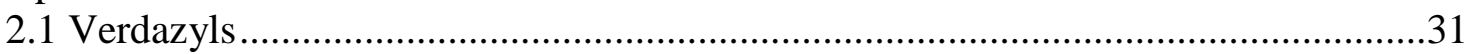

2.1.1 Previous Work ..............................................................................................

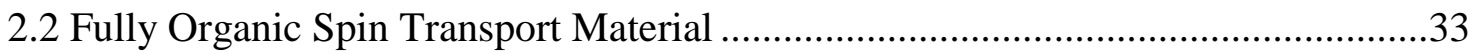

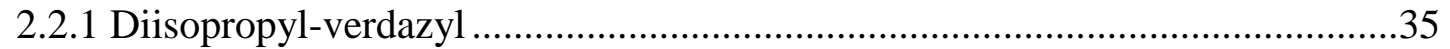

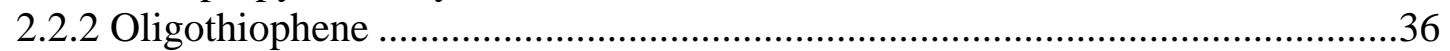

2.3 Iridium Borylation of Verdazyls ........................................................................

2.3.1 One Pot Double Suzuki .................................................................................

2.4 Characterization of Thiophene-Verdazyl species.....................................................40

2.5 Magnetism and Conductivity in Thiophene-Verdazyl species ...............................41

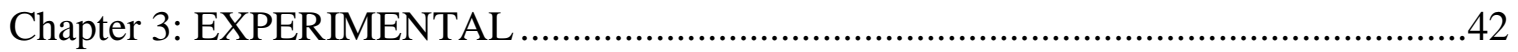

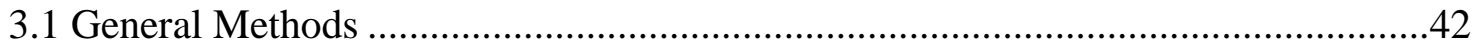

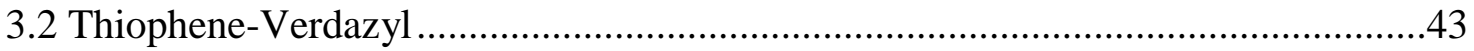

3.2.1 Preparation of 2,4-Diisopropyl-6-thiophene-1,2,4,5-tetrazane-3-one(3) ..........43

3.2.2 Oxidation to 1,5-Diisopropyl-3-thiophene-6-oxoverdazyl (4) ..........................44

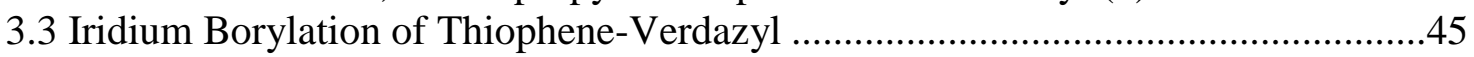

3.3.1 Synthesis of 1,5-Diisopropyl-3-thiophene-5-boronic acid pinacol ester-

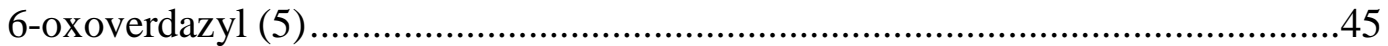

3.4 Alternative Route for Borylated-Thiophene-Verdazyl..........................................48

3.4.1 Synthesis of 5-Formyl-2-thienylboronic acid pinacol ester (3') .........................48 
3.4.2 Synthesis of 2,4-Diisopropyl-6-thiophene-5-boronic acid pinacol ester1,2,4,5-tetrazane-3-one (4')

3.4.3 Oxidation to 1,5-Diisopropyl-3-thiophene-5-boronic acid pinacol ester-

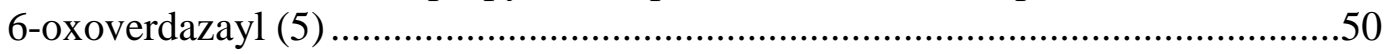

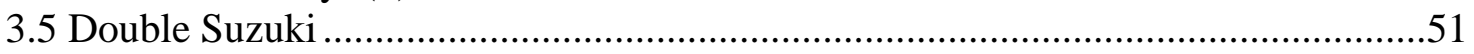

3.5.1 Synthesis of Bis (1, 5-diisopropyl-6-oxoverdazyl)-3,3'- $\alpha$ quaterthiophene (6)

3.5.2 Synthesis of Bis (1, 5-diisopropyl-6-oxoverdazyl)-3,3'-biphenyl-4,4'-

bithiophene (7).

Chapter 4: RESULTS \& DISCUSSION ..................................................................56

4.1 Failed Reactions \& Their Role in Designing Successful Reaction Routes ..............56

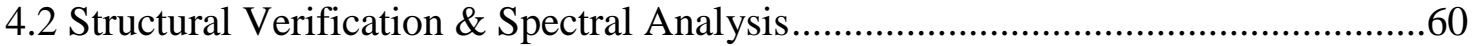

4.2.1: 2,4-Diisopropyl-6-thiophene-1,2,4,5-tetrazane-3-one (3) ...............................60

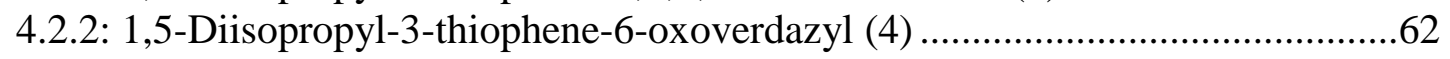

4.2.3: 1,5-Diisopropyl-3-thiophene-5-boronic acid pinacol ester-6-

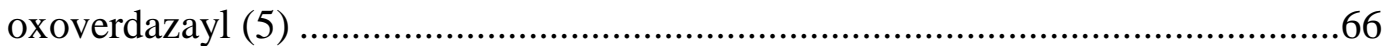

4.2.4: 2,4-Diisopropyl-6-thiophene-5-boronic acid pinacol ester-1,2,4,5-

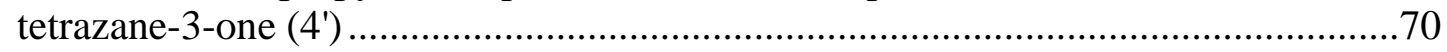

4.2.5: Bis (1, 5-diisopropyl-6-oxoverdazyl)-3,3'- $\alpha$-quaterthiophene (6) ...................73

4.2.6 Bis(1,5-diisopropyl-6-oxoverdazyl)-3-3'-bithiophene-4,4'-biphenyl (7)...........78

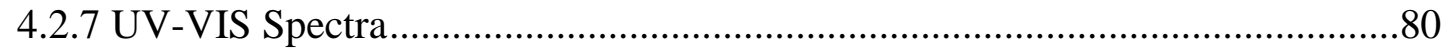

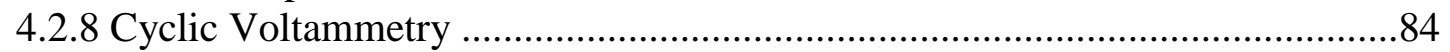

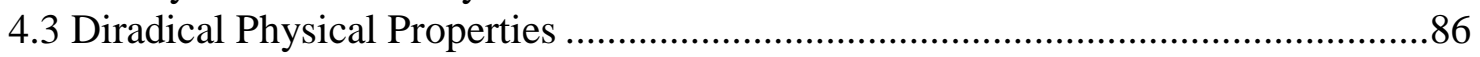

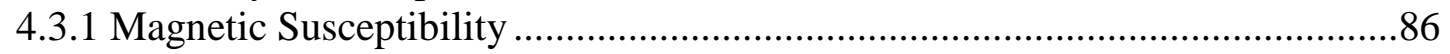

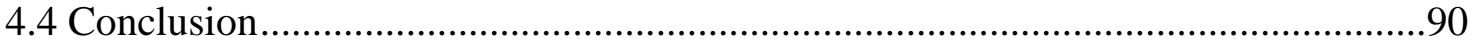

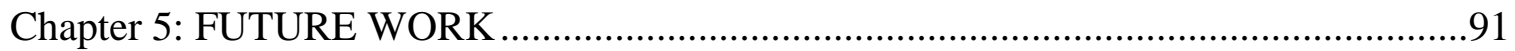

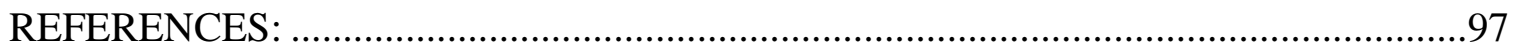

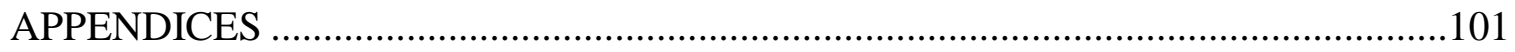

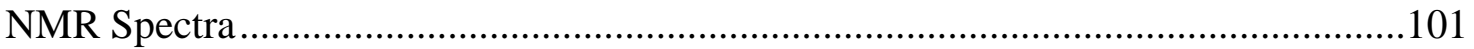

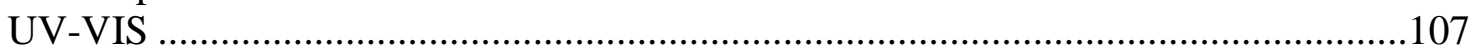

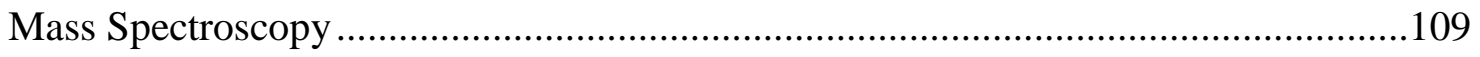

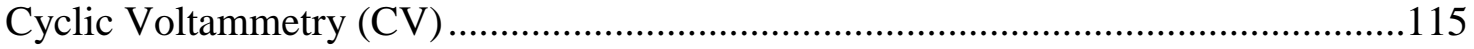

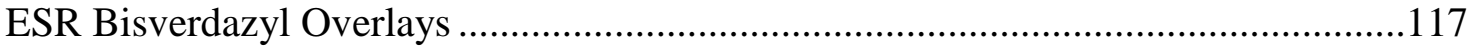

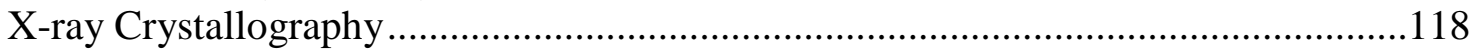




\section{LIST OF TABLES}

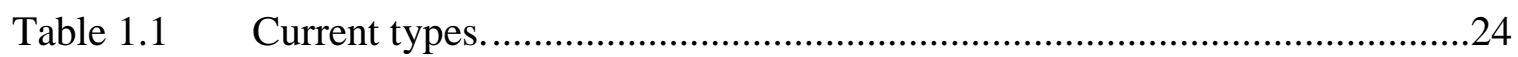

Table 4.1 UV-VIS peak maxima for radical species. ..........................................

Table 4.2 Cyclic voltammetry potentials for radical species................................85 


\section{LIST OF FIGURES}

Figure 1.1 Band gap of solid materials..........................................................

Figure 1.2 Doped semiconductors (N, P-Type) ..................................................

Figure $1.3 \quad$ Energy gap and doping in OSCs. ...................................................11

Figure 1.4 Electric and Magnetic force direction. ...........................................

Figure $1.5 \quad$ Types of magnetic materials.............................................................

Figure 1.6 Spin dependent electron scattering due to GMR .................................19

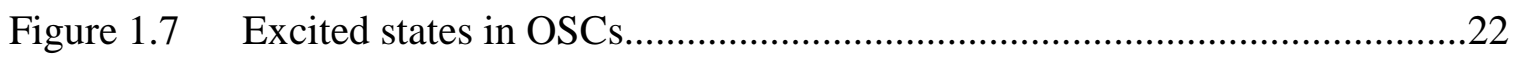

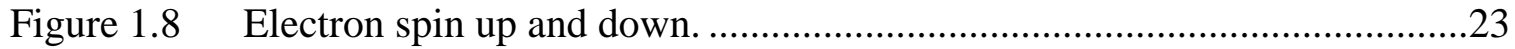

Figure 1.9 Spin polarization in non-magnetic, Ferromagnetic and

Ferromagnetic Half metal materials...............................................26

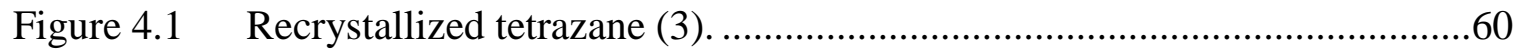

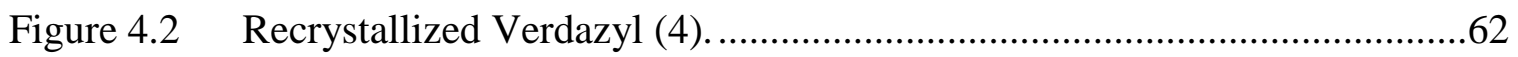

Figure 4.3 Verdazyl (4) Structure XRD......................................................65

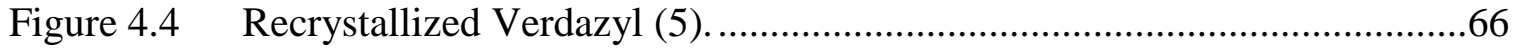

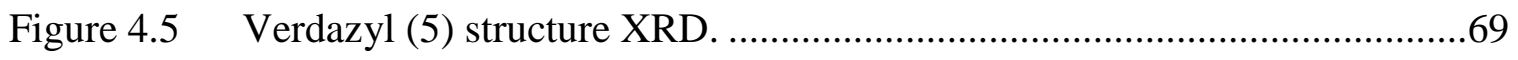

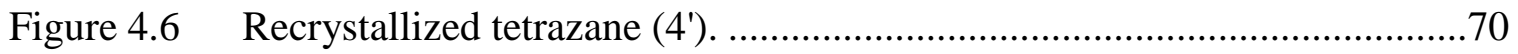

Figure 4.7 Recrystallized Bisverdazyl (6). ................................................... 73

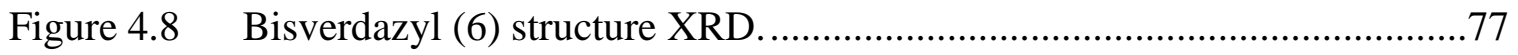

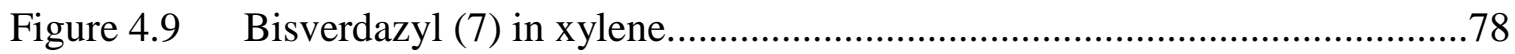

Figure 4.10 Electronic transitions due to UV-VIS absorption...................................81

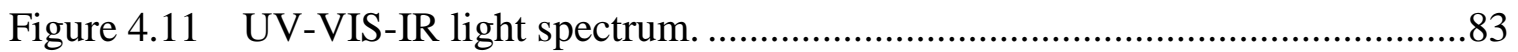

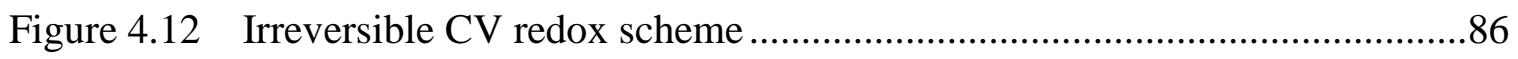


Figure $4.13 \chi \chi$ vs T Bis (1, 5-diisopropyl-6-oxoverdazyl)-3,3'- $\alpha$-quaterthiophene

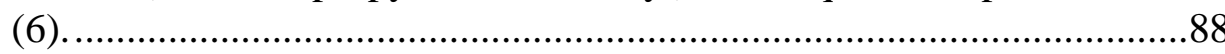

Figure 4.14 $\chi$ vs 1/T Bis (1, 5-diisopropyl-6-oxoverdazyl)-3,3'- $\alpha$ quaterthiophene (6)

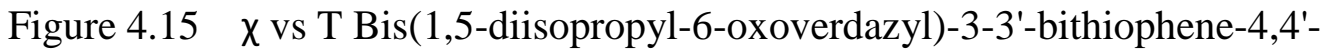
biphenyl (7).

Figure 4.16 $\chi$ vs 1/T Bis(1,5-diisopropyl-6-oxoverdazyl)-3-3'-bithiophene-4,4'biphenyl (7).

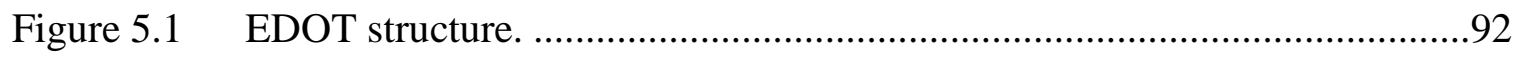

Figure 5.2 Intramolecular interactions in thiophene-EDOT oligomers........................93

Figure 5.3 Molecular packing motifs...................................................................94

Figure 5.4 Proposed spin propagation mechanism in conductive diradical..................96 


\section{LIST OF SCHEMES}

Scheme $2.1 \quad$ Oxoverdazyl resonance structures.................................................... 31

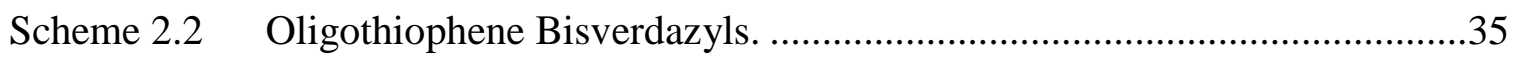

Scheme 2.3 General synthesis scheme for 1,5-Diisopropyl-6-oxoverdazyl................36

Scheme $2.4 \quad$ Iridum direct $\mathrm{C}-\mathrm{H}$ borylation reaction scheme.......................................39

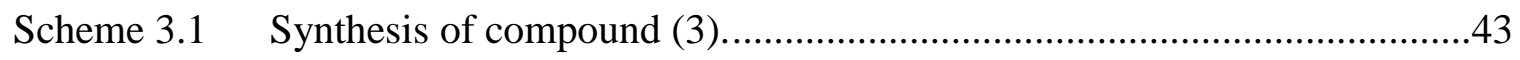

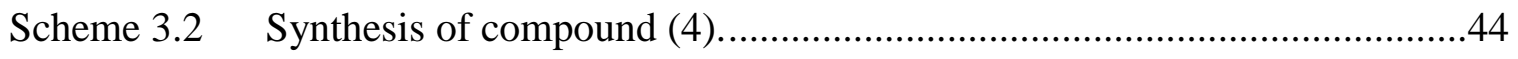

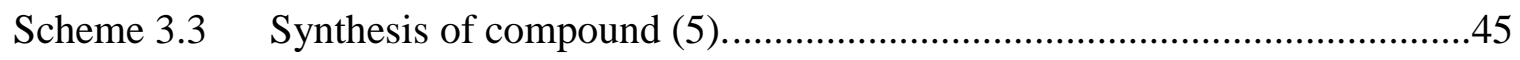

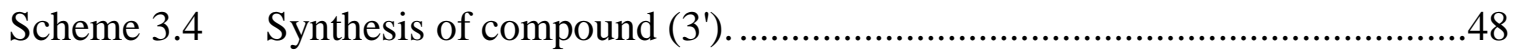

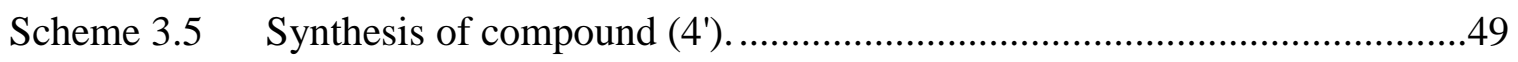

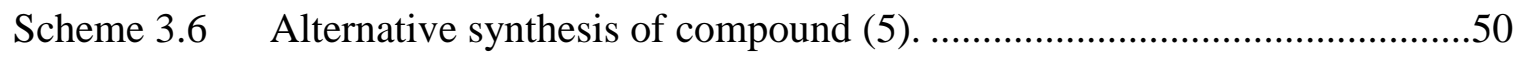

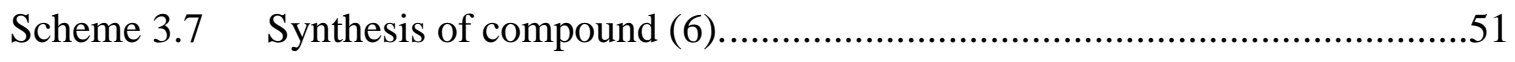

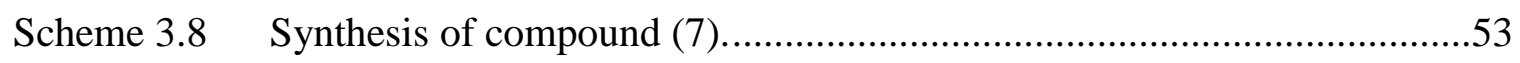

Scheme $4.1 \quad$ Failed double suzuki coupling ......................................................57

Scheme 4.2 Miyaura di(borylation) of dihalide...................................................59

Scheme 5.1 Synthesis plan for mixed thiophene/EDOT co-oligomer

Bisverdazyl system. .................................................................95 


\section{LIST OF SPECTRA}

IR Spectrum 1: 2,4-Diisopropyl-6-thiophene-1,2,4,5-tetrazane-3-one (3) \& 1,5-Diisopropyl-3-thiophene-6-oxoverdazyl (4)

ESR Spectrum 1: 1,5-Diisopropyl-3-thiophene-6-oxoverdazyl (4)............................64

IR Spectrum 2: 1,5-Diisopropyl-3-thiophene-6-oxoverdazyl (4) \& 1,5Diisopropyl-3-thiophene-5-boronic acid pinacol ester-6oxoverdazayl (5).

ESR Spectrum 2: 1,5-Diisopropyl-3-thiophene-5-boronic acid pinacol ester-6oxoverdazayl (5).

IR Spectrum 3: 2,4-Diisopropyl-6-thiophene-5-boronic acid pinacol ester1,2,4,5-tetrazane-3-one (4').

IR Spectrum 4: $\quad$ Bis (1, 5-diisopropyl-6-oxoverdazyl)-3,3'- $\alpha$-quaterthiophene

(6).

ESR Spectrum 3: Bis (1, 5-diisopropyl-6-oxoverdazyl)-3,3'- $\alpha$-quaterthiophene

(6).

IR Spectrum 5: $\quad$ Bis(1,5-diisopropyl-6-oxoverdazyl)-3-3'-bithiophene-4,4'biphenyl (7). .78

ESR Spectrum 4: Bis (1, 5-diisopropyl-6-oxoverdazyl)-3,3'-biphenyl-4,4'bithiophene (7). 


\section{Chapter 1: INTRODUCTION}

\subsection{Push for Novel Materials in Electronics}

Given the steady pace of technological advancements, the development and integration of electronic components utilizing materials with novel physical properties affording improved performance is a highly sought after goal. Indeed, the quest for ever greater processing power is best illustrated with Moore's law, a predicted trend envisioning a doubling of computing prowess every two years. Moore's prediction derived from his observation of increases in transistor count and efficiency. ${ }^{1}$ Remarkably, this rate of computing enhancement has largely held true in the decades following Moore's statement, but there are indications that the current practice of increasing component counts and downsizing may be reaching its physical and economic dead-end. Limitations imposed by quantum mechanics at the scales of a few nanometers are the primary culprit preventing further physical downscaling. Even more detrimental though, is the increasing cost associated with $R \& D$ required to maintain Moore's law trajectory of improved computational efficiency, decreasing the viability of products for mass market consumption with increasingly less affordable products.

Unsurprisingly, serious research is being carried out to develop new strategies for production of electronics that would sidestep the limitations of downsizing. The most promising approaches thus far can generally be placed into the three categories: 1 . enhanced integration of logic and memory, 2. novel device architecture, and 3. the introduction of devices with novel physical properties not exhibited in conventional CMOS devices. ${ }^{2}$ 
The greater integration of logic and memory is being pursued through the development of monolithic 3D multilayer memory/logic systems equipped with variable specialized memory types that allow for accelerated memory access and storage.

Research on new device architecture, on the other hand, seeks the implementation of novel designs and algorithms to overcome the so-called "Von Neumann bottleneck", that is the latency due to the sequential interaction between the processing unit and pre-stored device programming in memory units of conventional computing architecture ${ }^{3}$. Deep learning and neural algorithms, the latter inspired by neural functions in the brain, are particularly interesting alternatives to Von Neumann design. ${ }^{2,3}$ Finally, devices with material properties deviating from those of conventional CMOS devices are akin to the adhesive needed for the binding of the two areas discussed above. Some of the major benefits that may result from such devices are lower energy consumption and significantly lower device temperatures while under workload; the latter point is likely to be essential in the development of monolithic logic/memory systems.

One class of devices gaining much attention is spin transport electronics, or spintronics. Spintronic devices utilize electron spin in addition to the charge of electrons utilized in conventional electronic devices, giving rise to radically new physical properties and applications. Spintronic devices have already found commercial use, most notably in memory devices in the form of magnetic hard drive read heads through magnetoresistance effects. But research concerning spintronics is still in its early stages, with hints that field holds promise for much greater and far reaching applications in the sciences and electronics, from minor alterations to existing technology, to more radical 
applications such as quantum computing. ${ }^{4}$ The development of spintronic materials, or more precisely spin polarizing materials, is one of the main goals of the research discussed in this paper. But before delving into a detailed account of said materials and their synthesis, it's worth taking a look back at earlier electronic materials and devices, as well as fundamental concepts that would help elucidate our understanding of the science behind subjects discussed herein.

\subsection{Historical Overview of Electronic Components}

\subsubsection{Vacuum Tubes}

While strictly speaking, electric devices have existed for multiple centuries, electronic devices did not properly take off to become commercially available until the latter half of the 19th century with the advent of vacuum tubes. The most basic vacuum tube (or electron tube) designs consist of a filament acting as a hot cathode placed alongside an anode, both encased in an evacuated glass bulb. These devices function through thermionic emission, an effect in which thermally induced emitted electrons flow from a heated cathode to the anode; this process is assisted by the electric field between the anode and cathode. ${ }^{5,7}$ Additionally, the vacuum in the tube prevents the formation of ionized gas particles that would lead to irregular electrical current in the device. ${ }^{6}$

Incandescent light bulbs also utilize evacuated tubes, however unlike vacuum tubes which function through thermionic emission, incandescent light bulbs operate through thermally induced photon emission from a filament. Even so, the design of Incandescent light bulbs did play a part in development of vacuum tubes. Vacuum tubes came to dominate most electronic devices up to the mid-20th century. Many essential electronic 
components such as amplifiers, rectifiers, oscillation modulators, and various detection and switching devices saw their first implementations with vacuum tubes, ${ }^{7}$ and it is from these components that advanced electronic devices such and radio receivers, television (cathode-ray tubes), computers, and telephones were developed. Despite being supplanted in most electronic devices by later technology, vacuum tubes are still used in some specialized applications (e.g. tube guitar amplifiers).

\subsubsection{Gas Filled Tubes}

Concurrent to the advances with vacuum tubes, the similar but distinct gas-filled tubes (also known as discharge tubes) were developed. Gas-filled tubes are akin to vacuum tubes but with inert gases at low pressures $(10-55 \mathrm{mmHg})$. This apparently minor difference leads to significantly different electronic properties, as gas-filled tubes generally conduct a greater current due to the cascading ionization of the inert gas particles by the emitted electrons, which results in an increase in the charge carriers received at the anode. On the other hand, this also leads to reduced control over electrons in gas-filled tubes compared to vacuum tubes. ${ }^{6}$

Gas-filled tubes fall into two categories of cold cathode or hot cathodes, with the primary distinction relating to whether the cathode is heated to induce thermionic emission (hot cathode) or that emissions are caused by natural background radiation in the environment (cold cathode). Because cold cathode gas tubes can maintain constant voltages, they have been used as voltage regulators, polarity indicators, electronic switches, and radio frequency field detectors, to name a few. Hot cathode gas-filled tubes, on the other hand, have primarily found use as rectifiers and electric switches. ${ }^{6}$ 


\subsubsection{Solid State Electronics}

The next major revolution in electronics came about from the introduction of transistors. By the mid-20th century electronic devices began to shift away from electron tubes (vacuum tubes/gas-filled tubes) to solid state devices utilizing semiconductors. Conventional inorganic semiconductors are primarily made of silicon, germanium, and their respective oxides and alloys. Early development of semiconductors preceded solid state electronics for some time, but it was the utility of semiconductors in transistors that began the shift to solid state electronics. A more in-depth discussion of the nature of semiconductors will take place in section 1.3 , but here it will suffice to mention that semiconducting materials in transistors allow for more advanced switching and amplifying functions in comparison to electron tube technologies. In parallel to the development of transistors, electronic devices began to be fabricated as integrated circuits (IC) where components are packed closely on top of a semiconductor sheet.

In comparison to earlier electronic devices with discrete components, IC devices have a considerably reduced power consumption and smaller device sizes. In practical terms this means commercially available solid state electronic devices experience less frequent defects and could carry out more advanced operations at lower costs, both in terms of materials and power consumption, at successively smaller scales. ${ }^{7}$ We will next discuss some physical theories and properties that help explain the pre-eminence of semiconductors in modern electronics and how the development of organic semiconductor materials may further enhance the development of solid state devices. 


\subsection{Band Theory}

Electrical conductivity is the degree to which a substance allows the movement of charge carriers. Electrical conductivity is dependent on both the charge carrier concentration and the mobility of charge carriers in each substance. The conductivity of solid phase materials falls into the three categories of insulators, with little to no electrical conductivity; conductors, with a high degree of conductivity, and semiconductors with moderate and tunable conductivity.

We can begin to understand how conductors, insulators and semiconductors differ in their conductivity by looking the simplest electronic configurations in the form of atomic orbitals. Atomic orbitals are discrete quantum mechanically allowed energy states for electrons bound by an atomic nucleus. If instead of individual atoms we regard a slab of solid phase matter with innumerable closely packed atoms and or molecules, we can begin to imagine the sum of the atomic and molecular orbitals forming a continuous band of allowed energy states. Band theory, posits that the lower electron energy band, the valance band describes the energy states for outer shell (valence) electrons bound by individual atoms and molecules of the bulk material, while the more energetic band, the conduction band, describes the energy state of electrons unbound from individual atoms or molecules and instead delocalized across the bulk material. When speaking of electrical conduction and the passage of electrical current, we are in fact speaking of the free movement of electrons or other charge carriers across the conduction or valence band. The variance in electrical conduction for various materials is due to both the band gap, the region of forbidden electron energy states separating the conduction band and 
valence band, as well as the availability of empty energy states in the valence band which may act as electron acceptors. Figure 1.1 visualizes the differences in band gap of conductors, semiconductors and insulators.

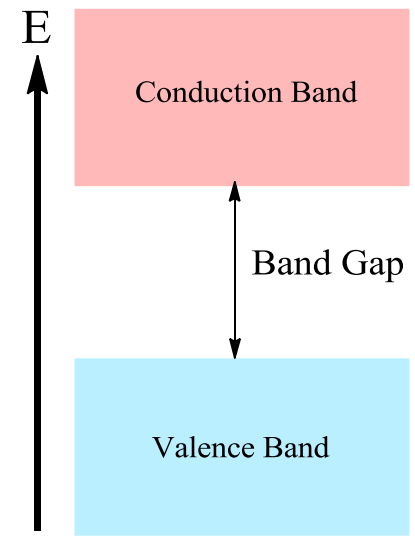

Insulator

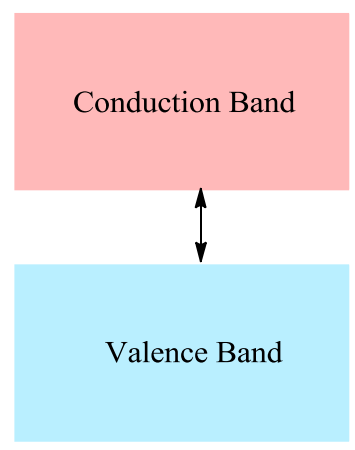

Semiconductor

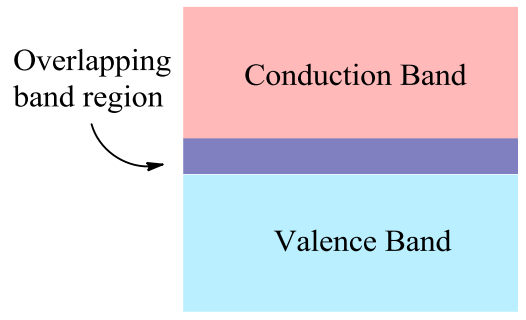

Conductor

Figure 1.1 Band gap of solid materials.

Conductors such as metals exhibit an overlapping of the valence and conduction band, thus, the absence of a band gap allows for high electrical conduction. What this means is that valence electrons in conductors can readily be delocalized to the conduction band as the energy requirements are close to or on par with that of valence band.

Insulators on the other hand have a large band gap, such that under ordinary circumstances the energy demands for electron transition from valence to conduction band are too great to overcome, resulting in very little or no electrical conduction. Semiconductors have band gaps that are sufficiently small enough to allow electrical conduction under certain circumstances and can act as both insulators and conductors through modifications. ${ }^{8}$ 
When an electron is excited from the valence band to the conduction band through absorption of an appropriate amount of energy, the previous electron state is made vacant forming an electron-hole. While electron-holes mimic the behavior of positively charged particles, electron-holes aren't actual charged particles with mass, but rather the absence of a negatively charged electron in an allowed electron state of the valence band. This electron absence leads to a positive charge imbalance, as the positive charge of the nucleus now exceeds that of negative charges arising from electrons. The ultimate result of these effects is that the electron adjacent to a hole moves to fill the electron-hole, resulting in the generation of a new electron-hole in the electron state just vacated. This process is repeated giving way to the movement of electrons in the opposite direction of the electron hole movement. It can be understood then that electrical conduction does not occur only due to movement of electrons promoted to the conduction band, but can also result from the formation and movement of electron-holes in the opposing direction to electrical current. $^{8}$

While free electrons are the primary charge carriers for conductors, with semiconductors (and insulators) the generation of charge carriers begins through the excitation of an electron to the conduction band, forming free electrons and electronholes. If the electron excitation falls short of the energy requirements to form free charge carriers (electron or hole), a bound electron-hole pair or exciton can form. Excitons in part can undergo dissociation to form free electrons and holes with the absorption of energy. In general, semiconductor fall into the two categories of intrinsic and extrinsic. Intrinsic semiconductors are made of pure crystalline semiconducting substances such as 
silicon, and exhibit an equal number of electrons and holes. Extrinsic semiconductors in contrast have added impurities from hetero-atoms. These impurities or dopants cause localized excess or deficiency of electrons in the crystal structure of the semiconductor leading to unequal populations of electrons and holes in extrinsic semiconductors. If the added dopants cause an excess of electrons, acting as electron donors, it will be an n-type semiconductor and electrons become the majority charge carrier. We see the inverse picture with p-type semiconductors, which have an excess of electron-holes and dopants acting as electron acceptors, leading to electron-holes becoming the majority charge carrier. The valency of dopants used for extrinsic semiconductors is entirely reliant on the semiconductor material and the type of (n or p-type) extrinsic semiconductor desired.

The importance of the distinction between Intrinsic and extrinsic semiconductors is illustrated in Figure 1.2.
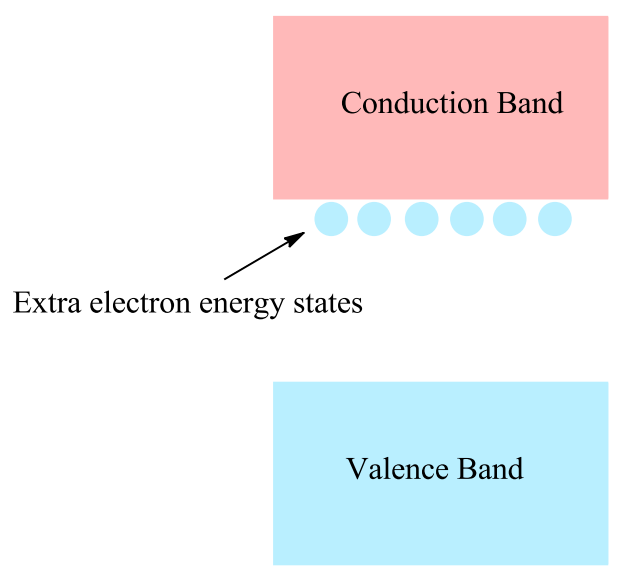

N-type Doping

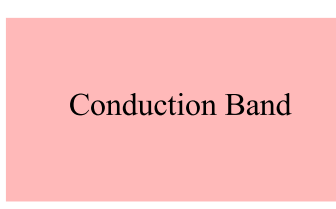

Extra hole energy states

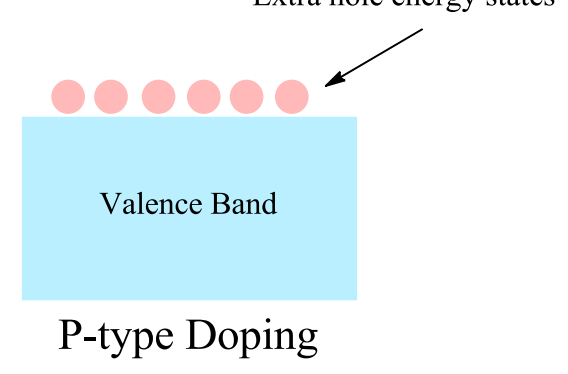

Figure 1.2 Doped semiconductors (N, P-Type).

With n-type semiconductors the addition of dopants yields excess electrons with energy states much closer to the conduction band (smaller effective band gap) than 
intrinsic semiconductors, while p-type semiconductors give excess electron holes with energy states much closer to the valence band. Thus, both n-type and p-type Extrinsic semiconductors experience significantly greater electrical conduction compared to Intrinsic semiconductors, as charge carrier concentrations are elevated, while effective band gaps are reduced. ${ }^{8}$

\subsection{Organic Semiconductors}

Thus far our discussion of semiconductors has been focused on conventional inorganic semiconductors made of silicon, germanium, and their derivatives. But certain types of organic compounds can also act as semiconductors. Organic compounds consisting of hydrocarbons with $\pi$-bonded conjugated systems were first discovered to exhibit semiconductivity in the early 1970s. ${ }^{10}$ These organic semiconductors (OSC) come in two variants of elongated conjugated polymers or smaller conjugated molecules. In both forms, the semiconducting property stems from the delocalization of electrons across the conjugated structure due to the $\pi$-bonds from overlapping of $p$ orbitals in $s p^{2}$ hybridization. ${ }^{9}$ However, while the forces involved in holding inorganic semiconductors together are strong covalent bonds, OSCs are held together through weaker Van Der Waals intermolecular interactions. This is more pronounced in smaller conjugated molecules, as the sheer number of interactions in larger polymers bestows strong overall intermolecular forces. The intramolecular conduction of conjugated systems increases with more extensive conjugation length (see top portion of Figure 1.3). This is because a more extensive conjugated length shrinks the effective band gap. It is worth noting though that conjugation length is not simply an elongation of the molecular structure, but 
rather an elongation of a continuous delocalized $\pi$-bond system, and thus any discontinuity resulting from molecular geometry or bond order change will lead to a larger band gap and reduce conductivity. ${ }^{11,12}$

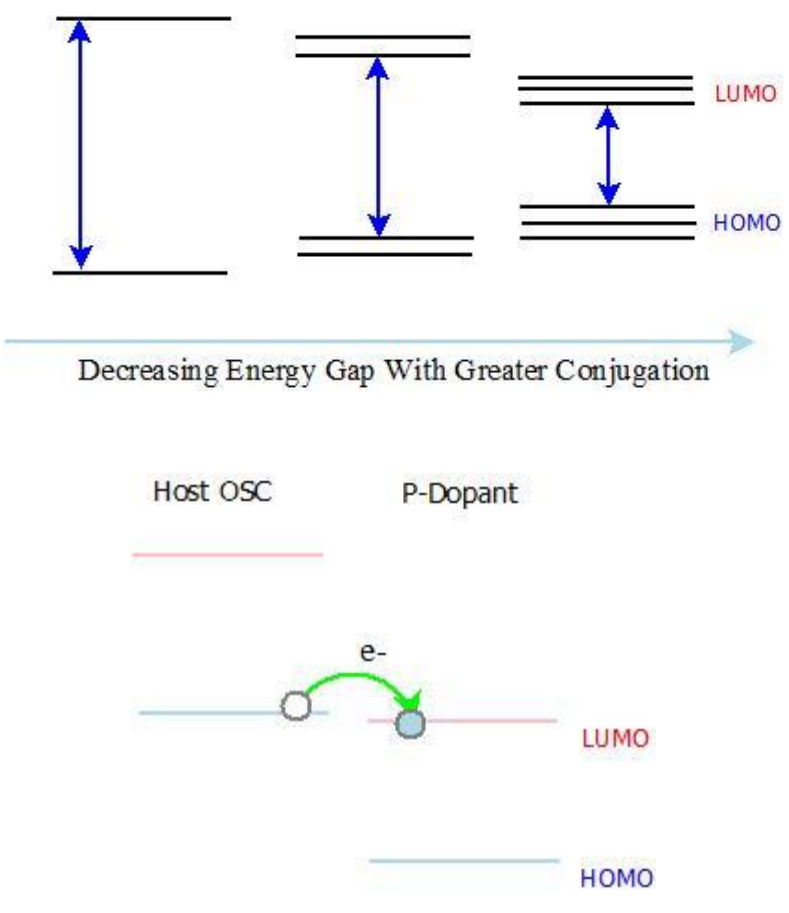

Figure 1.3 Energy gap and doping in OSCs.

With OSCs the highest occupied molecular orbital (HOMO) and lowest unoccupied molecular orbital (LUMO) are discrete energy levels analogous to inorganic bulk semiconductor valence and conduction bands respectively. And just like inorganic semiconductors, OSC conductivity can be increased through doping by introducing impurities in the form of molecular segments (functional groups and or moieties) that can act as either acceptors or donors towards the primary organic semiconductor (see bottom portion of Figure 1.3). ${ }^{10}$

While both organic and inorganic semiconductors exhibit tunable conductivity due to their semiconducting properties, there are a few key differences between the two material 
types worth mentioning. First, organic semiconductors generally have larger band gaps and thus lower conductivity than their inorganic analogues. The primary reason for the larger organic band gaps is the weaker intramolecular forces of such substances. A secondary reason for the larger effective band gaps (specifically the transport band gap) in organic semiconductors is the higher exciton binding energy of organic materials. In semiconductors when an electron is excited to a more energetic state through thermal excitation or light absorption, depending on the magnitude of energy transferred, it may form a free electron and hole or a bound electron-hole pair (exciton), the latter of which has a lower energy state than an unbound free electron and hole. Exciton binding energy is the energy required to untether the electron and hole pair. The magnitude of the exciton binding energy is greater in organics compared to inorganics because of the lower dielectric constant of hydrocarbons, as the charge densities in carbon and hydrogen are comparatively less polarizable. This is clearly shown in the equation bellow, where the exciton binding energy $\left(\mathrm{E}_{\text {exciton }}\right)$ is shown to have an inverse relationship with the square of the dielectric constant $\left(\varepsilon_{\mathrm{r}}\right)$, while being proportional to Rydberg's unit of energy (Ry). Additional terms in said equation include the reduced mass $(\mu)$, and mass of a free electron $\left(\mathrm{m}_{\mathrm{e}}\right)$.

$$
\mathrm{E}_{\text {exciton }}=-\frac{1 \mu}{\varepsilon_{\mathrm{r}}^{2} \mathrm{~m}_{\mathrm{e}}} \mathrm{R}_{\mathrm{y}}
$$

The greater exciton binding energy of organic materials also highlights the appreciable difference between optical gap and transport gap in organic semiconductors. 
The optical gap is the energy required to form a bound exciton, while the transport gap accounts for the energy required to form free electrons, in other words the transport gap is the optical gap plus the exciton binding energy. As a result, in inorganic semiconductors the difference between transport and optical gaps are negligible due to small exciton binding energies, while organic semiconductors exhibit higher exciton binding energies, such that the difference between optical and transport gaps is significant, and one factor leading to larger band gaps in organic materials. ${ }^{13}$

A second major difference between OSCs and inorganic semiconductors is the mechanism of charge transport. As previously discussed, inorganic semiconductors consist of tightly packed, covalently bonded atoms characterized by ordered structures, where the charge carriers are transported across the entire bulk seamlessly in the conduction or valence band with a high degree of charge mobility. While more ordered OSCs with single crystal structures can exhibit similar band type charge transport mechanisms as inorganic semiconductors at lower temperatures (with charge movement between HOMO/LUMO levels), under more typical conditions with higher temperatures and less ordered morphology, OSCs experience more localizing effects, such that charge carriers are more limited to individual molecules and intermolecular (and to some degree intramolecular) charge transport takes on an incoherent hopping mechanism, which greatly diminishes charge mobility in comparison to inorganic semiconductors. ${ }^{14}$

Considering the often-greater conductivity and charge carrier mobility of inorganic semiconductors compared to OSCs, one might question the viability of OSCs as an alternative to conventional inorganic semiconductors. But organic semiconductors outdo 
their inorganic counterparts with some very useful properties. Because organic semiconductors are synthesized on a molecular level, the chemical structure of OSCs is highly tailorable and only limited by the compatibility of desired functional groups and the structural stability of the molecule. This means OSCs' chemical and physical properties can be adjusted through synthetic alterations. Similarly, because OSCs are made of organic molecules with weaker intermolecular forces, they are considerably more flexible and resistant to breakage than inorganic semiconductors, a feature that has already found widespread use in recent electronic devices in the form of flexible organic light emitting diodes (OLED). Additionally, many common organic semiconductors can be synthesized from cheap precursors and fabricated through solution processing, making OSCs commercially competitive with inorganic semiconductors in some areas. ${ }^{13}$

In the context of this paper though, perhaps the most important feature differentiating OSCs from inorganic semiconductors is weaker spin-orbit coupling (SOC) in organics. Spin-orbit coupling is a quantum mechanical property that results from the interaction of an electron spin with its orbital motion under potential. This interaction is very important for the spin conservation of electrons, as will be further elaborated on in section 1.6.1. The weaker SOC interactions of organics are due to the elements hydrogen, carbon and nitrogen (primary atoms in organic semiconductors) being relatively low mass atoms at the top the periodic table. The further down the periodic table we go the greater the SOC effects as the nucleus has a greater positive charge and its influence on outer electrons is stronger. Thus, heavier inorganic semiconducting materials such as silicon and germanium experience comparatively stronger SOC than their organic counterparts. ${ }^{15}$ 
The weaker spin-orbit coupling of OSCs plays an integral role in maximizing the performance of said materials in devices utilizing electron spin which is a focus of the research discussed here.

\subsection{Magnetism}

Although magnetism is a commonly known phenomenon, its useful to review the basic theory of magnetism and its various types before we proceed to discuss the lesser known phenomenon of magnetoresistance. Magnetism in its simplest form can be described as attractive or repulsive forces applied to substances when in the presence of a magnetic field. A magnetic field itself arises from the passage of electrical current, which produces a magnetic field perpendicular to the direction of the electrical current. Figure 1.5 (left) gives a visual representation of the right-hand rule and the directional relation between the magnetic force, magnetic field, and electrical current. However, magnetism is only one component of electromagnetism. The collective forces due to electricity and magnetism are referred to as the Lorentz force. The other component of the Lorentz force is the electric force and its direction is determined by the direction of the electric field and charge of the object the field is acting on, as illustrated by the right half of Figure 1.4.

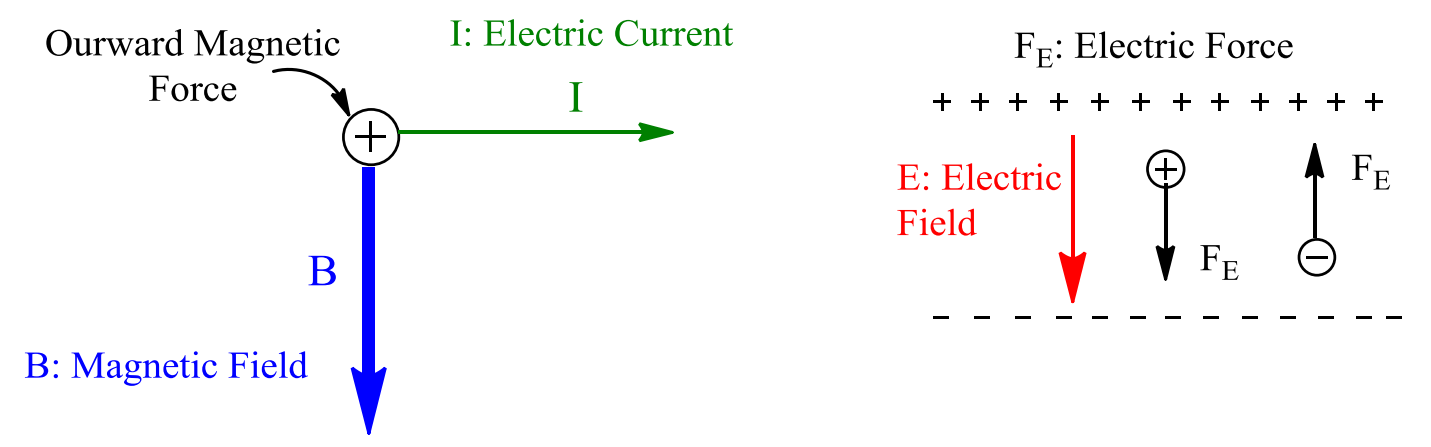

Figure 1.4 Electric and Magnetic force direction. 
Just as electricity can produce magnetism through the passage of current, so too can magnetism produce electricity by moving a magnetic field across an object. The close link between electricity and magnetism is further highlighted by the fact that they are not observed as separate physical properties. ${ }^{16}$

Of course, not all materials interact with a magnetic field in the same manner, instead the behavior of materials under a magnetic field is determined by the electron spin arrangement of a substance and therefore there are different types of magnetic materials. In Figure 1.5 we can see the five common types of magnetic materials.

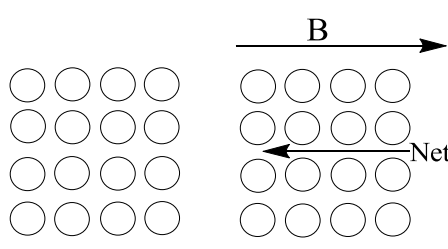

Diamagnetic

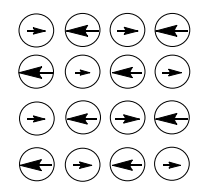

Ferrimagnetic

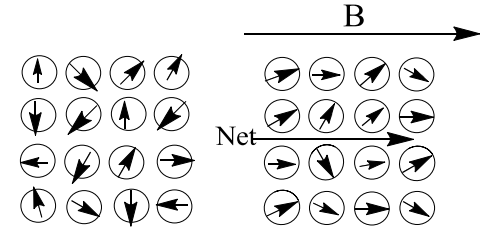

Paramagnetic
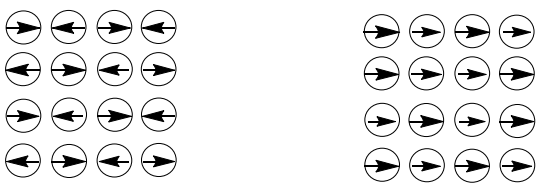

Antiferromagnetic

Ferromagnetic

Figure 1.5 Types of magnetic materials.

With diamagnetic materials, all electrons are paired with one another resulting in a net zero magnetic moment when unexposed to a magnetic field. Under a magnetic field diamagnetic materials exhibit a weak temporarily induced magnetic dipole and the substances experience a repellant force with respect to the magnetic field and are said to experience negative susceptibility. Paramagnetic materials contain unpaired electrons which are randomly oriented due to thermal motion, resulting in zero magnetic moment 
in the absence of a magnetic field. In the presence of a magnetic field though, paramagnetic dipoles tend to align in the direction of the field, experiencing an attractive force and positive susceptibility. Ferromagnetic materials exhibit the strongest form of magnetism, and indeed this type of magnetism is often perceived as synonymous with magnetism. In ferromagnetic materials, a significant number of electrons are not only unpaired, but also aligned such that the collective electron moments in ferromagnets produce a high degree of overall material magnetism. Under a magnetic field ferromagnetic materials are strongly aligned with the field, and have a large positive susceptibility, and may adopt permanent dipoles. Furthermore, in ferromagnets the spin magnetic moment and the orbital magnetic moment both align, which is one factor that leads to the strong magnetism of ferromagnetic materials. Antiferromagnetic materials also have unpaired electrons, but they are arranged in such a manner that the dipoles cancel out one another giving zero net magnetization. The last type of magnetic material, Ferrimagnetic materials, stand in between ferromagnetism and antiferromagnetism by similarly exhibiting ordered magnetism, but while in antiferromagnetic materials the opposing magnetic dipoles are equal in magnitude, ferrimagnetic materials have unequal

opposing dipoles giving rise to a non-zero magnetization. ${ }^{16,17}$ Of course, a given material can exhibit more than one type of magnetism in different domains of a substance.

\subsubsection{Magnetoresistance}

In certain materials, an applied external magnetic field can alter the electrical resistance of the material, this effect is called magnetoresistance. Magnetoresistance can be both positive or negative in value, with positive values indicating an increase in 
resistance and lowering of electrical current; while negative magnetoresistance will lower resistance and increase current. Magnetoresistive effects can largely be classified into five types, two of which are observed in single layer systems with a single type of magnetic material, while the other three are observed with multilayer systems with alternating layers with differing forms of magnetism. ${ }^{18}$

\section{A. Single Layer Systems}

1. Ordinary magnetoresistance (OMR) is observed in non-magnetic (diamagnetic) metals, where the repulsion of the electrons from the magnetic field due to the Lorentz force causes a positive increase in electrical resistivity proportional to the magnetic field strength. While OMR values are smaller than some other types of magnetoresistance, OMR does increase considerably at lower temperatures due to the lessening of thermal phonon scattering. ${ }^{18}$

2. Anisotropic magnetoresistance (AMR) is observed with ferromagnetic materials and is highly dependent on the angle of the applied magnetic field with respect to the direction of electrical current. AMR is at its lowest when the magnetic field is perpendicular to the electrical current and at a maximum when the field is parallel to the current. The mechanism responsible for this form of positive magnetoresistance is thought to be electron scattering effects arising from spin-orbit interactions. ${ }^{18}$

\section{B. Multilayer systems}

3. Giant magnetoresistance (GMR) is a quantum mechanical effect observed in multilayer systems with alternating ferromagnetic and non-magnetic layers. The magnitude of GMR is increased when the ferromagnetic layers have anti-parallel 
magnetization with one another, while GMR effect is lowered with parallel ferromagnetic layers. Figure 1.6 shows why GMR provides differing degrees of resistivity with antiparallel and parallel FM layer arrangements.

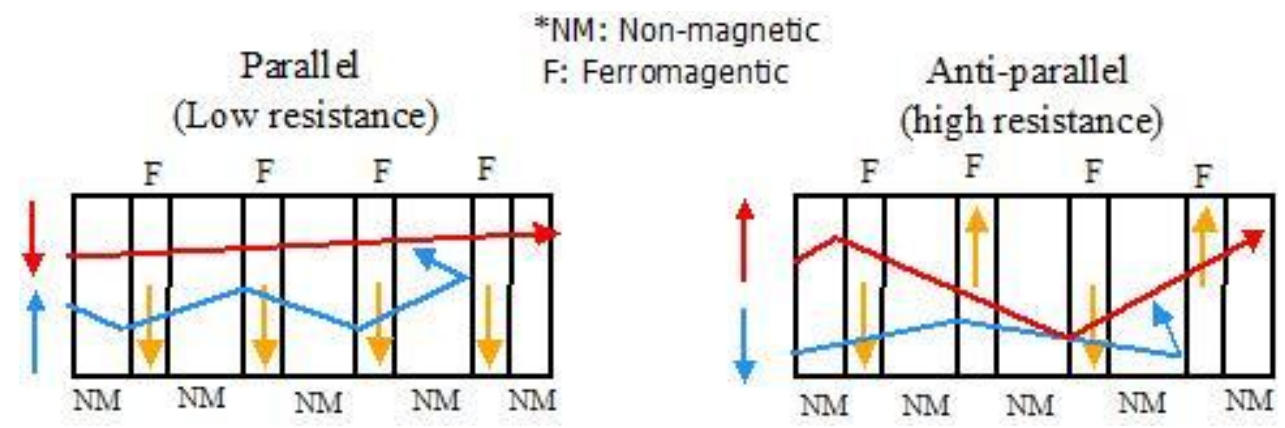

Figure 1.6 Spin dependent electron scattering due to GMR.

Anti-parallel ferromagnetic arrangements lead to greater electron scatter, as electrons with spin magnetic moments anti-parallel to magnetic dipoles in the FM layer will undergo more scattering, while electrons with the parallel spin will pass through unimpeded. As can be seen anti parallel layers will inevitably lead to more overall scattering of electrons irrespective of electron spin. ${ }^{18} \mathrm{GMR}$ can be used to spin polarize current by favoring the transmission of one electron spin type, as seen in parallel FM layer arrangement of Figure 1.6.

4. Tunneling magnetoresistance (TMR) is in many regards similar to GMR, but instead of alternating FM and non-magnetic layers, TMR occurs at a junction where two FM layers (electrodes) are separated by a thin insulating layer, collectively referred to as a magnetic tunnel junction. In TMR, electrons travel from one FM electrode to the other by tunneling through the insulating barrier while conserving their spin. Similar to GMR, anti-parallel magnetized FM layers generate high TMR, while parallel layer 
magnetization results in lower TMR, and just as with GMR, this process can be used to to generate spin polarized current. ${ }^{18}$

5. Colossal magnetoresistance (CMR) has thus far only been observed in manganite perovskites with magnetoresistance values that far exceed that of other magnetoresistance types. The mechanism behind CMR is still an open debate, but among the theories proposed are FM to paramagnetic phase transitions and electron phonon coupling. ${ }^{18}$

We can see that a major application of the various types of magnetoresistance is electron spin manipulation, more specifically magnetoresistance can be used to increase the proportion of one electron spin compared to the other, a process termed spin polarization or spin filtering. Spin polarization is an essential property in the field of spintronics and will be further discussed in section 1.6.

This brings us to one additional magnetoresistance type most relevant to this paper, organic magnetoresistance (OMAR). This type of magnetoresistance can be regarded as a subset of GMR but it differs from GMR in utility of organic semiconductors. OMAR can be generated through two different methods. The first is to have two FM layers with an organic semiconductor layer in between and inject spin polarized current through the FM electrode and have the OSC layer act as the spin transporting layer. The second more nascent method involves the application of a magnetic field directly to an OSC layer. This method does not utilize FM electrodes, instead non-magnetic conductive contacts are used, and the OMAR effect arises directly from the organic semiconductor itself. ${ }^{18}$ The novel materials developed in our research are most likely to be employed in the second OMAR method. Interest in OMAR does not simply arise from its surprisingly 
high magnetoresisance values, the ability of organic semiconductors to maintain a longer spin relaxation lifetime due to their weaker spin-orbit coupling and hyperfine interactions in comparison to inorganic FM materials, has arguably been an even more exciting prospect. This provides a great advantage to OMAR and organic semiconductors in the field of spintronics, and is why such materials are being researched more vigorously in recent years.

An important mechanism that prolongs spin transport in OSCs arises from the formation of triplet excited states. As can be seen in Figure 1.7, excitation of electrons or excitons in OSCs can lead to either a singlet or triplet excited state. The Pauli exclusion principle state that no two electrons can have identical quantum configuration, so that for a given spatial quantum number only two electrons of opposing spin are allowed. Additionally, according to the spin selection rule, electron spin is conserved when undergoing radiative transitions between excited and ground states. A singlet excited state consists of two electrons of higher and lower energy states with opposite spin, thus the transition of the higher energy electron to the lower energy state is allowed at a rapid rate as the resulting ground state will have two paired electrons of opposite spins, satisfying the selection rule. A triplet excited state however has two electrons of higher and lower energy states but with the same spin and so the transition of triplet excited electron to the ground state is forbidden. 


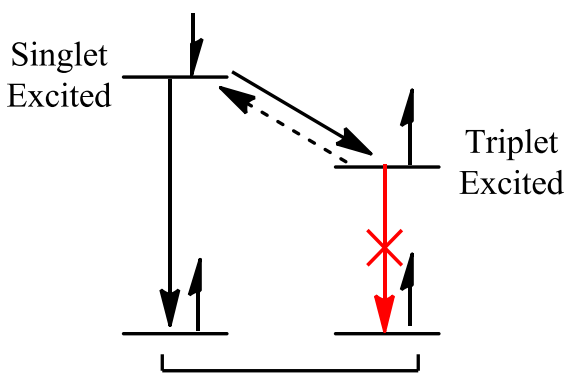

Ground State

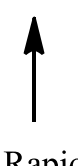

Rapid

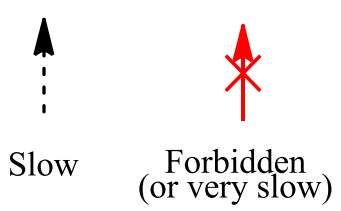

Forbidden
(or very slow)

Figure 1.7 Excited states in OSCs.

In order for an electron in the triplet excited state to transition to the ground state it typically undergoes the much slower non-radiative intersystem crossing transition to a singlet excited state, resulting in spin flipping of the excited electron before the transition to singlet ground state is allowed. This means that the generation of triplet excited electrons or excitons leads to a much longer spin lifetime and as such very beneficial in circumstances where the maximization of spin lifetime is desired. Once again this phenomenon is observed to a greater extent in OSCs because, the weaker spin-orbit coupling of organics leads to fewer intersystem crossing events that would allow the transition from triplet to singlet excited states. Of course, OSCs can be modified to generate more singlet excited states if greater recombination emission is desired. This tailorability is a major selling point of OSCs, and why OSCs have found plentiful use in both photovoltaics and OLED devices. ${ }^{18}$

\subsection{Spintronics}

There have already been several mentions of electron spin earlier in this paper, but we have yet to discuss the precise nature of this concept. Electron spin is short for the 
intrinsic spin angular momentum of an electron. Electron spin is analogous to the angular momentum of a rotating charged macroscopic sphere about an axis, and much like its macroscopic analogue, electrons spin generates a magnetic moment. Unlike macroscopic objects though, sub-atomic particles such as electrons are governed by quantum mechanical properties, and one aspect of this is that electron spin is a fixed quantized value rather than the seemingly continuous range of magnitudes seen with everyday macroscopic measurements.

The electron spin quantum number $m_{S}$, is the quantum mechanical description of electron spin and alongside the principle quantum number $n$, angular momentum quantum number $l$, and magnetic quantum number $m_{l}$, complete the electron quantum numbers, which quantum mechanically describe the positioning and motion of an electron. ${ }^{19}$ Electrons can only have two possible spin orientations with $m_{S} \pm 1 / 2$ for spin up and spin down (see Figure 1.8), and no two electrons in a given system can have the same exact quantum numbers, including the spin quantum number. This means that if two electrons share the same value for the other three quantum number $\left(n, l, m_{l}\right)$, they must at least have opposite spin quantum numbers $(+1 / 2 \&-1 / 2)$.

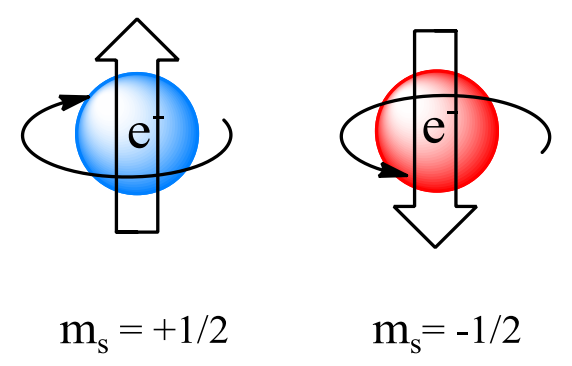

Figure 1.8 Electron spin up and down. 
The utility of electron spin and its associated physical properties has given rise to the burgeoning field of electron transport electronics, or spintronics. Before discussing how spintronics works, its useful to learn the different types of current with respect to electron spin population, as visualized in Table 1.1.

Table 1.1 Current types.

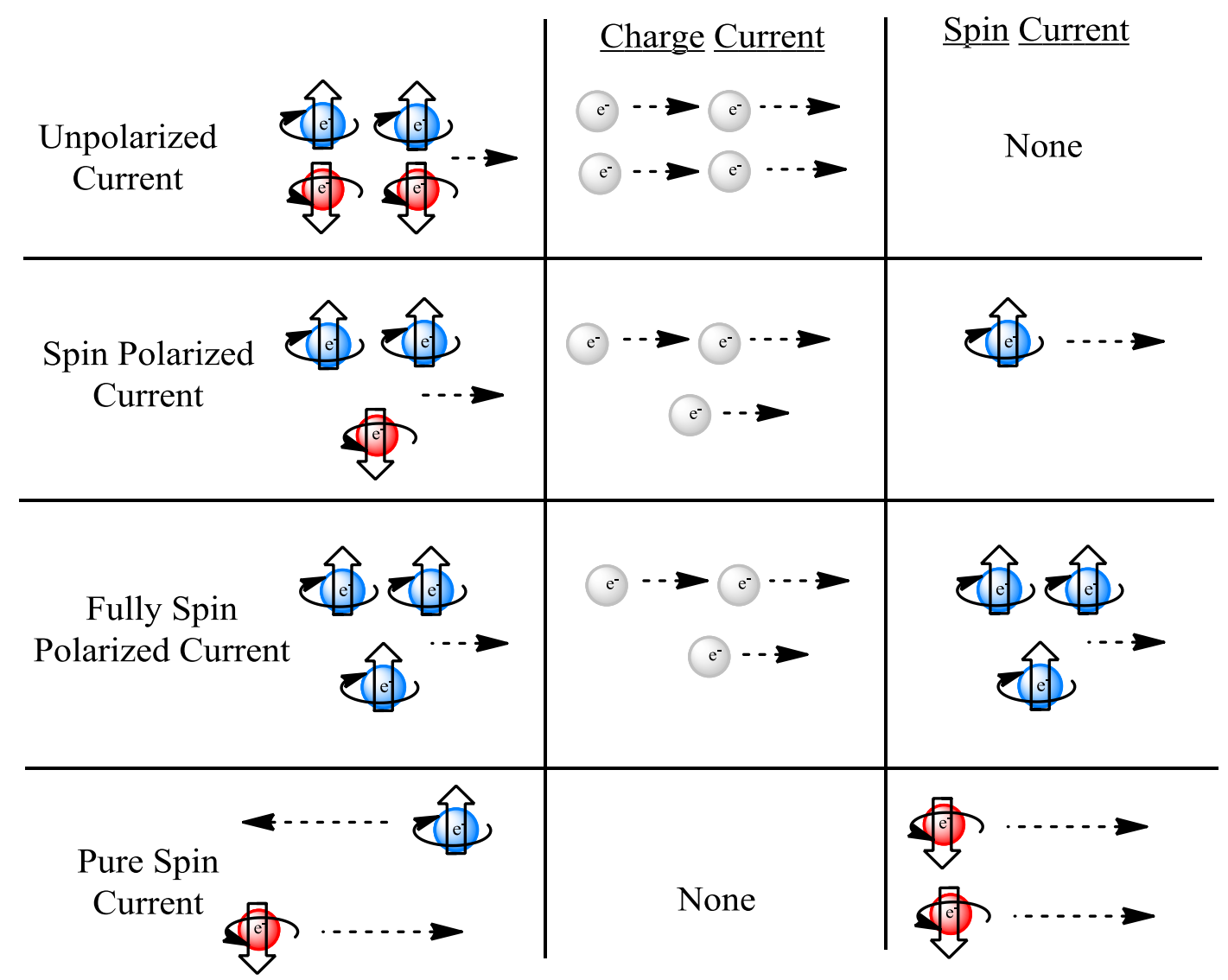

Regular unpolarized electrical current used in conventional electronics, has an equal population of both up and down spin electrons, but when the population of up and down spin electrons is unequal, the current is said to be spin polarized, with the higher population spin acting as the majority spin and the lower population spin type is refered to as the minority spin. ${ }^{20}$ Electrical current can be spin polarized to a variable degree, but 
if the current is only populated with one spin form, the current is said to be fully spin polarized, although in reality a small minority spin component is likely to still persist. In special circumstances, it is possible to generate pure spin current, which unlike other spin polarized current has no net charge transfer and is effectively only the transportation of electron spin. Pure spin current manifests when an equal number up spin electron and down spin electrons move in opposite directions such that the transfer of charge is zero, but there is transport of up spin electrons in one direction and transport of down spin electrons in the opposite direction. Pure spin current is particularly interesting as it eliminates electron charge as a variable and allows for the sole utility of electron spin to the exclusion of electron charge.

The importance of current spin polarization lies in the fact that the magnetic properties of electron spin can only be employed with an imbalance of electron spin orientations, as otherwise the equal number of opposing spins counteract the magnetism of one another. Much like classical electromagnetism demonstrates the close link between electricity and magnetism, in spintronics, a close link is shown between electron charge and its spin, with methods such as the spin Hall effect and inverse spin Hall effect, which allow for the conversion of electrical current to spin current and vice versa respectively. ${ }^{22}$

Some of the methods used to generate spin polarized current have already been discussed in section 1.5.1 when discussing various types of magnetoresistance effects, which are closely intertwined with spintronics. In this section, we will instead address the 
process involved in generation of spin (polarized) current in more general terms (see Figure 1.9).

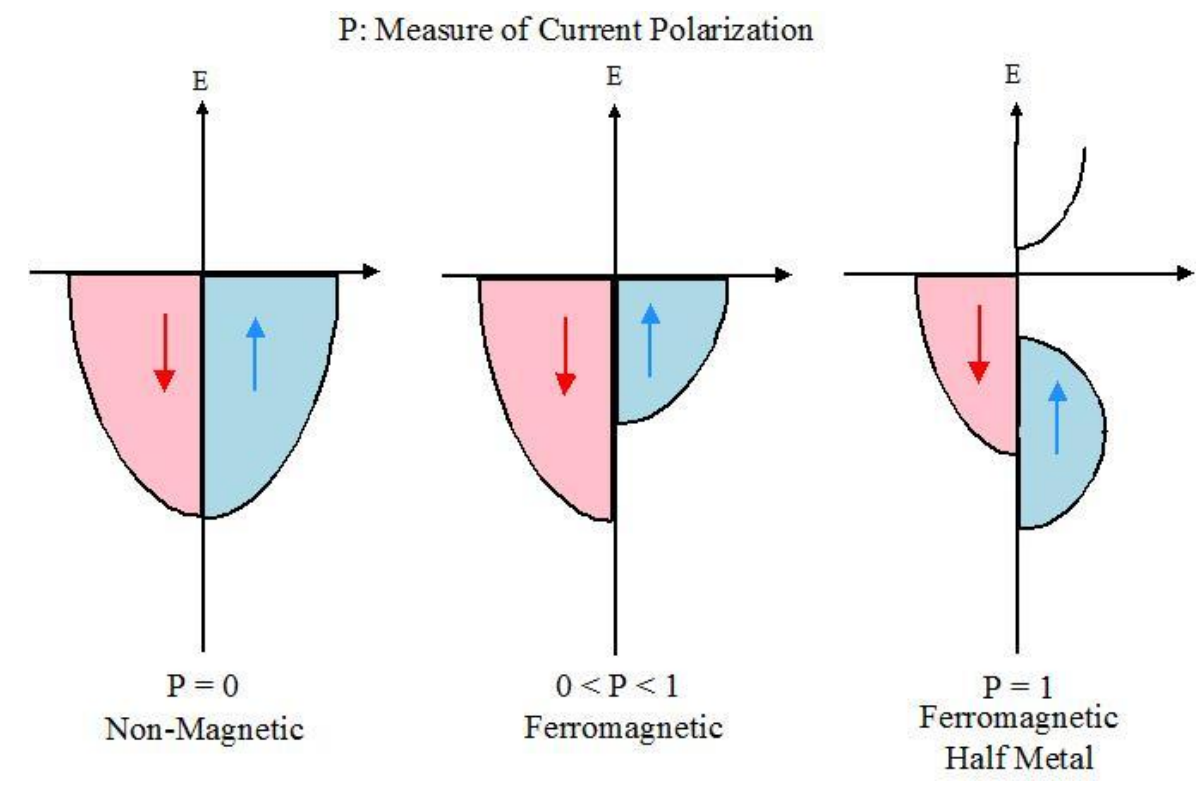

Figure 1.9 Spin polarization in non-magnetic, Ferromagnetic and Ferromagnetic Half metal materials

Non-magnetic materials have an equal preference for the conduction of either electron spin, and so any current passing through non-magnetic substances will have its spin population unperturbed. Magnetic materials, especially those displaying ferromagnetism exhibit greater conductivity for one spin over the other, thus, any current passing through magnetic materials will undergo at least some degree of spin polarization. Full spin polarization may occur with materials that behave like a conductor to one spin type, while behaving like an insulator or semiconductor to the opposing spin, these materials termed half-metals, can generate currents that are largely populated by only one spin type. ${ }^{20}$ 
The field of spintronics has already yielded many applications for the use of spin polarized current, a full account of which is beyond the scope of this thesis. However, possibly the most promising aspect of spintronics is its low power demand. In conventional electronics, power consumption has steadily risen as components have continued to downsize, but as the primary functionality of spintronic devices derive from the use of electron spin rather than the movement of electron charge, the former of which requires much less energy, comparatively spintronic devices require much less power to operate. The lower power consumption also side-steps some of the heat related wear associated with high power electronics, thus allowing more durable devices with less need for cooling systems. Furthermore, the lower power usage of spintronic devices also eases the physical limitations imposed on component downsizing observed in conventional electronics. ${ }^{21}$ Even so, the field of spintronics still faces a number of challenges that have prevented it from supplanting conventional electronics. Two major challenges are the short lifetime of spin polarized current and its diffusion length. Spin relaxation lifetime is the duration for which spin polarized current endures before the inevitable loss of its polarization due various scattering and dephasing effects arising from spin-orbit coupling and hyperfine interactions. Spin diffusion length is the distance spin polarized current can traverse before the loss of polarization. One might be inclined to believe that spin diffusion length is directly proportional to spin relaxation lifetime, but this neglects to consider spin mobility. It is very much possible for a substance to have long spin lifetimes but low spin mobility, such that spin diffusion lengths are modest. Indeed, this has been the case for many organic materials used for spin transport. ${ }^{20}$ 
Organic materials experience long spin relaxation lifetimes due to weak spin-orbit coupling, but due to the less ordered structure and morphology of many OSCs, such materials have low spin mobility and less impressive spin diffusion lengths. Inorganic semiconductors on the other hand often have low spin relaxation lifetimes, but high spin mobility and spin diffusion. An ideal spin transport material would then possess characteristics of both inorganic and organic semiconductors, with both high spin diffusion lengths and long relaxation lifetimes. Researchers have sought to make advances in this regard by either developing hybrid organic-inorganic materials, or wholly organic materials with improved spin mobility.

\subsection{Free Radicals}

Free radicals are atoms or molecules with one or more unpaired electrons. In most circumstances having unpaired electrons leads to extremely reactive species, because the octet rule is not met and the singly occupied molecular orbital (SOMO) holding the unpaired electron is in an elevated energy state. ${ }^{23}$ Because of this characteristic, most free radicals are very short lived species that rapidly react with other compounds or undergo decomposition. However, under some rare conditions free radicals can be stable. Generally, the more an unpaired electron is delocalized across a molecule the more stable it becomes. What this means, is that radicals with a higher degree of substitution and/or conjugation tend to be more stable. Additionally, as a trend, electron donating functional groups can contribute to radical stabilization by reducing the degree of electron deficiency of a radical. Interestingly though, the use of both electron withdrawing and electron donating functional groups in conjugation with one another has been found to 
stabilize radicals even further due to the captodative effect, where the combination of the two groups allows for better delocalization of the radical electron cloud due to resonance. To a lesser degree, radicals may also be stabilized through steric effects, as bulky groups may prevent the access of potentially reactive compounds to the radical site.

A major utility of stable free radicals is the indirect detection of unstable radicals. This process is called spin trapping and it involves a specially designed ionic precursor (spin trap) that can bind to a reactive radical to form a spin adduct. Spin adducts are stable, or more persistent radicals, which can be detect and characterized through the radical spectrometry technique, electron spin resonance spectroscopy (ESR). ${ }^{24}$ The five major families of stable free radicals are nitroxides, dithiadiazolyls, semiquinones, phenoxyls, perchlorotriphenylmethyls, and verdazyls. ${ }^{25}$

Stable organic free radicals have many other uses beside spin trapping, much of which derives from the open shell nature of radicals. The most relevant property of stable radicals for this paper is the magnetism imparted by unpaired electrons. In non-radical organic compounds with fully paired electrons, the spin of each electron is counteracted by the opposite spin of the electron it is paired with, which is why non-radical organics do not demonstrate magnetism other than the weak diamagnetism present in all substances. But with unpaired electrons as in the case of radicals, the spin of the unpaired electron bestows a degree of paramagnetism to the radical species by creating a magnetic moment. This effect is often enhanced when coordinating radicals with transition metals which have more unpaired electrons available in their unfilled d-orbitals, ${ }^{26}$ and as previously discussed, materials with magnetism can exhibit magnetoresistance, and thus 
can contribute to spin polarization. Because of these characteristics, the development of systems combining organic semiconductors with stable organic free radicals is seen as one possible route to developing wholly organic materials for the field of spintronics. 


\section{Chapter 2: RESEARCH GOAL}

\subsection{Verdazyls}

Having discussed some generalities on stable radicals, we will now narrow our discussion to a single class of stable radicals most relevant to our research, the verdazyls. Verdazyls are among the most versatile of stable free radicals, and this versatility is largely due to the R-group substituents at the 1, 3 and 5 positions of the verdazyl ring. Despite this, verdazyls have been studied to a lesser extent than the better known nitroxide free radicals, even though nitroxides have fewer positions available (1-2) for substitution compared to verdazyls ( 3 positions). The primary means by which verdazyl radicals are stabilized is through delocalization of the unpaired electron across all four nitrogens of the verdazyl central ring, and this is supported through ESR measurements, which reveal nearly identical hyperfine coupling for the four nitrogens. The delocalization of verdazyl radicals is demonstrated in the four resonance structures seen in Scheme 2.1. ${ }^{29}$<smiles>[R]c1nn(C)c(=O)n([R])c1=O</smiles>

Scheme 2.1 Oxoverdazyl resonance structures. ${ }^{31}$

\subsubsection{Previous Work}

A fair amount of research has already gone into developing systems incorporating verdazyls for spintronic applications. A major highlight in this area has been hybrid organic-inorganic systems consisting of transition metals complexes with verdazyl 
containing ligands. Hicks et al. reported the synthesis of a few verdazyl-transition metal complexes. ${ }^{27}$ In this study, the verdazyl-metal coordination complexes exhibited strong magnetic interactions between the transition metal center and the radical ligands. The magnetic interactions between the verdazyl ligands and the transition metals ranged from ferromagnetism to antiferromagnetism, with the transition metal element playing a defining role in the observed magnetic behavior. The magnetic interactions in the verdazyl-transition metal complexes arise from the orbital overlap between the SOMO orbitals of the verdazyl ligands and the d-orbitals of the transition metals. Interestingly, in cases where the radical SOMO orbital is orthogonal to that of the transition metal, ferromagnetic exchange is observed, while the absence of orthogonality generally leads to antiferromagnetic interactions. The strength of the observed ferromagnetic exchange interactions seen with some of the verdazyl-transition metal complexes sets them apart from other stable radical-transition metal complexes, which exhibit comparatively weaker interactions. ${ }^{27}$ In a closely related paper from Barclay et al., the synthesis of a similar verdayl-metal complex is explored, however in this case, tridentate ligands are utilized in place of the bidentate ligands seen in the Hick et al. paper mentioned above. It's noteworthy that the coordination of two tridentate verdazyl containing ligands with a nickel metal center led to an even stronger ferromagnetic interaction than that of previously reported verdazyl-metal complexes, which may suggest a greater importance for ligand denticity. ${ }^{28}$

Several additional verdazyl coordination systems have since been prepared with different metal centers including the transition metals $\mathrm{Cu}$ (I, II), $\mathrm{Ni}$ (II), $\mathrm{Mn}$ (II), $\mathrm{Ag}(\mathrm{I})$, 
$\mathrm{Zn}(\mathrm{II})$, and $\mathrm{Ru}(\mathrm{III})$, as well as lanthanides such as $\mathrm{Tb}^{+3}, \mathrm{Dy}^{+3}$ and $\mathrm{Gd}^{+3}$, though lanthanides were found to have weaker magnetic exchange interactions compared to transition metals due to their coordination through carbonyl oxygens. ${ }^{29}$ The surprisingly strong magnetic interactions of verdazyl-metal complexes make them attractive candidates for further development in the context of spin polarization. But spin polarization is only one aspect of spintronics, and the development of efficient spin transporting materials is just as important. Therefore, rather than synthesizing verdazylmetal complexes, we have instead sought to synthesize entirely organic systems that join the magnetic properties of verdazyl stable radicals with the conductive properties of organic semiconductors, to better serve as spin transporting materials.

\subsection{Fully Organic Spin Transport Material}

Organic semiconductors can take the form of either long conjugated polymers or smaller conjugated molecules. The two OSC forms excel in different ways, OSC polymers typically have higher electrical conductivity and structural strength due to their more extensive conjugation lengths, but as polymers they also have lesser defined structures because of the inherent variation in polymer length. Furthermore, polymers are often insoluble or poorly soluble in common solvents unless substituted with extensive pendant groups, which may alter the electronic and conductive properties of said polymers. OSC molecules, including conjugated oligomers, may exhibit more modest conduction than conjugated polymers, but they make up for this with well-defined identical structures because of controlled syntheses, which also allow for precise structural modifications. Additionally, because of the shorter length of conjugated 
molecules, they are less prone to solubility issues than polymers, and require few or no pendant groups for complete dissolution. Considering the drawbacks and advantages of the two OSC types, conjugated molecules were judged to better serve our research goals, as the mild loss in conductivity is easily outweighed by features such as, greater synthetic freedom and control, easier characterization, and solution processing.

The synthesis of conjugated molecules from individual aryl groups is well established with various named coupling reactions. However, since our intended product contains radical moieties, a greater selectivity is required to ensure the compatibility of reaction conditions with verdazyl stability. To this end, the Brook research group sought to establish the compatibility of Suzki-Miyaura coupling for cross-coupling reactions involving aryl-verdazyls in their 2017 publication. ${ }^{30}$ Suzuki coupling reactions are particularly convenient coupling reactions, as the reaction is comparatively eco-friendly and can be carried out in relatively mild basic conditions with easily obtained reagents. In the Brook group publication, a series of Suzuki cross-coupling reactions utilizing either halogenated (I, Br) aryl-verdazyls, or borylated aryl-verdazyls gave decent yields, except for a small number of reactants that contained bulkier substituents, or functional groups that bind competitively to palladium. ${ }^{30}$

Given the ground work lain by the Brook research group in the Le et al. paper, we intended to build on this research by utilizing Suzuki coupling reactions to synthesize bisverdazyl diradical end-capped conjugated oligomer systems, with oligothiophenes acting as the bridging conjugated oligomer as seen in Scheme 2.2. 


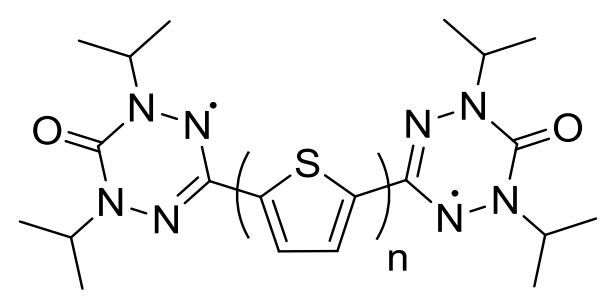

Scheme 2.2 Oligothiophene Bisverdazyls.

In the subsequent sections of this chapter, we will discuss the synthesis strategy and characterization methods we employed for this class of radicals.

\subsubsection{Diisopropyl-verdazyl}

Verdazyls come in a few variants depending on the substituent at the 6 position of the verdazyl ring. Oxo-verdazyls have a carbonyl substituent at 6 position, and are favored for being less prone to unwanted disproportionation side reactions. ${ }^{29}$ All verdazyls types can be further stabilized through their substituents at $1 \& 5$ positions. Stabilizing substituents can either be aromatic or branched alkyl groups. Aromatic substituents increase the delocalization of the radical, while bulky alkyl groups increase steric effects which decrease reactivity. Since our target molecules will contain a conjugated oligomer, solubility of the target molecule requires consideration. To this end, substitution of the 1 and 5 positions on oxo-verdazyl with isopropyl groups will not only assist in further stabilization of the verdazyl radical, but also aid in counteracting the stacking effect of the oligothiophene chain, and in the process, improve the diradical solubility in common organic solvents. The synthesis route for 1,5-diisopropyl-oxoverdazyls is shown in Scheme 2.3, and follows the procedure detailed in the Paré et al. paper, with minor modifications. ${ }^{31}$ 


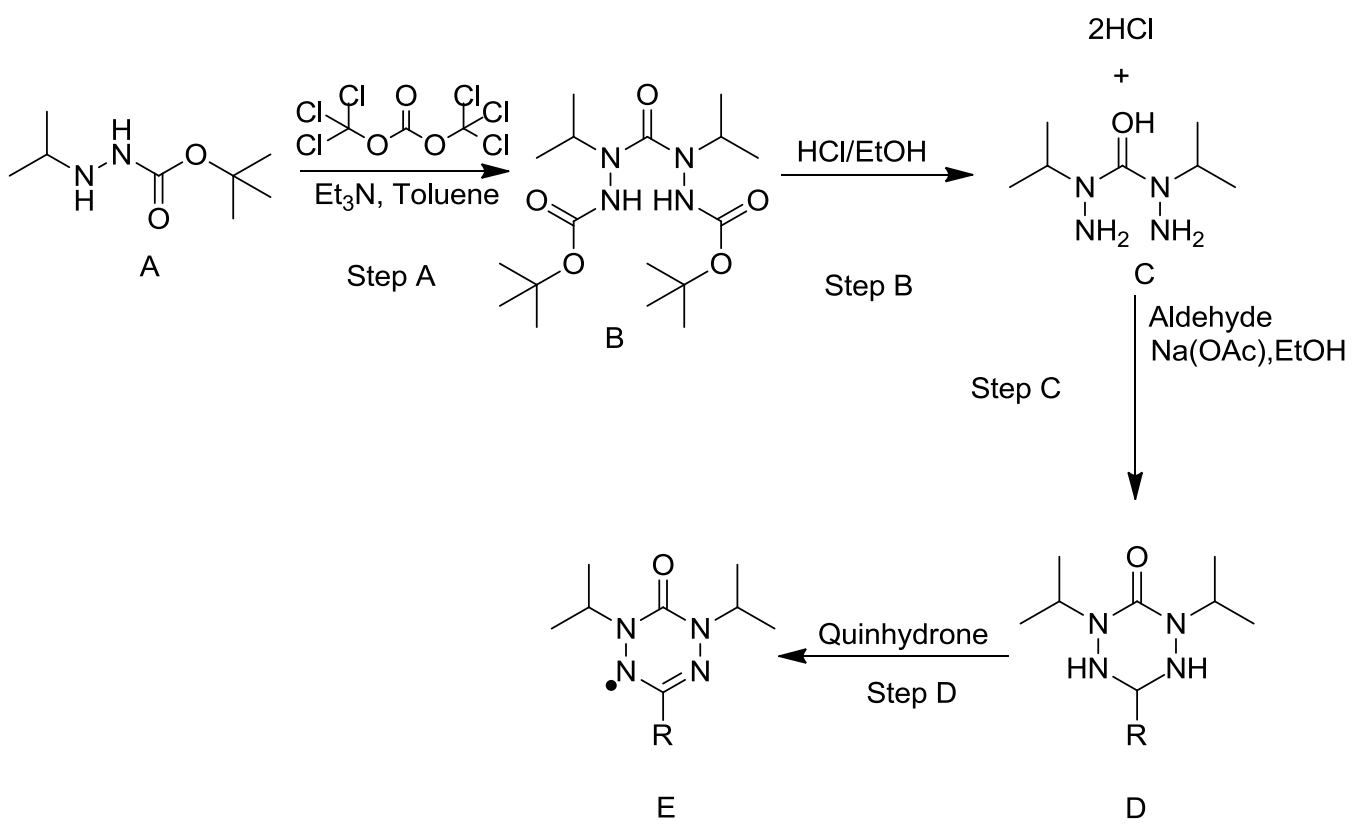

Scheme 2.3 General synthesis scheme for 1,5-Diisopropyl-6oxoverdazyl. ${ }^{31}$

\subsubsection{Oligothiophene}

Thiophene is a heterocyclic five membered aromatic ring containing a sulfur.

Thiophenes are comparatively electron-rich heterocycles because of the electron donating property of sulfur. As with all aromatic compounds, thiophene is a planar molecule, and conjugation across its polymer and oligomer forms is maximized by keeping the thiophenes in the same plane, and this is best achieved through $\alpha$ - $\alpha$ ' linkage of the thiophenes. In comparison to benzene, thiophene is less aromatic, with a smaller resonance energy. Surprisingly though, oligo- $p$-phenylene and poly-p-phenylene are generally less conductive than their thiophene counterparts, as benzene $p$-oligo/polymers have slightly twisted structures due to the repulsive effects of the ortho hydrogens of adjacent benzene rings, which reduce the planarity of the overall structure and its degree 
of conjugation. ${ }^{32} \alpha$-Oligo/polythiophenes on the other hand are quite planar and thus experience greater conjugation than their $p$-phenyl analogs. Additionally, thiophenes are also more reactive in the context of electrophilic substitution because of their electron rich nature. ${ }^{33}$ In most circumstances substitution at 2, 5 positions of thiophenes is favored over the 3, 4 position, making the synthesis of $\alpha$-oligothiophenes considerably more straightforward than that of benzenes, where substitution at para, meta and ortho positions requires more careful synthetic planning, all of which make oligothiophenes a more attractive candidate for our synthesis work.

While poly/oligothiophenes are recognized for their conductivity in doped form, polythiophenes and oligothiophenes have also gained much attention for properties related to thiophene-thiophene conformations, which bestow a wide range of interesting physical and chemical properties. Polythiophene has thus far been employed in photovoltaics, organic field effect transistors, and various optical applications. Oligothiophenes have been noted for possessing many of the same properties as their polythiophene analogues, ${ }^{34}$ This makes oligothiphenes very attractive materials, so much so that even if our intended bisverdazyl-oligothiophene target molecule exhibits modest spin transporting properties, investigation of the molecule for additional properties will be well worth the effort.

The synthesis of the oligothiophene chain will consist of a series of halogenation and borylation reactions to obtain the necessary reagents for subsequent Suzuki coupling reactions, which will join the thiophenes until the desired oligothiophene length is obtained. ${ }^{35}$ Here we have not pursued oligothiophene lengths greater than that of 
tetramers, as even short oligothiophenes are noted for their poor solubility. The incorporation of diisopropyl-verdazyls is expected to enhance the solubility of the oligothiophene, but even so, we believe a quaterthiophene to be a fine initial compromise between oligomer length and solubility.

\subsection{Iridium Borylation of Verdazyls}

We previously discussed the work done by the Brook research group in the Le et al. publication, on the application of Suzuki Miyaura coupling for aryl-verdazyls. ${ }^{30} \mathrm{We}$ believe we can build on this study in the area concerning the preparation of boronatearyl-verdazyls. In Le et al. paper, the boronate-aryl-verdazyls are prepared via Miyaura borylation, a closely related reaction to Suzuki coupling, that utilizes aryl halides and boronate sources in a palladium catalyzed reaction to form aryl-boronates. ${ }^{36}$ While this reaction serves its purpose well, the synthesis of a boronate-aryl-verdazyl from an arylverdazyl would require 2 steps if Miyaura borylation is employed. First halogenation of the aryl group, second the Miyaura borylation step itself. Iridium catalyzed direct C-H borylation is a well-known alternative borylation method, which allows the direct addition of a boronate functional group to arenes, without the need for a halogenation step (see Scheme 2.4). ${ }^{37}$ Thus, the major secondary synthesis goal of our research here, is to carry out this type of iridium catalyzed borylation on aryl-verdazyls and confirm the compatibility of this reaction type for verdazyls. The accomplishment of this goal is significant as it will cut down on the number of reaction steps required for the synthesis of verdazyl-conjugated oligomer systems and in the process increase the yield of the final product. It is even suggested that success in this area may lead to the development of in 
situ alternating borylation/Suzuki coupling reactions, that could allow the synthesis of longer chains without the need for intervening workup and purification steps, sharply reducing material costs, processing time and increasing yield.

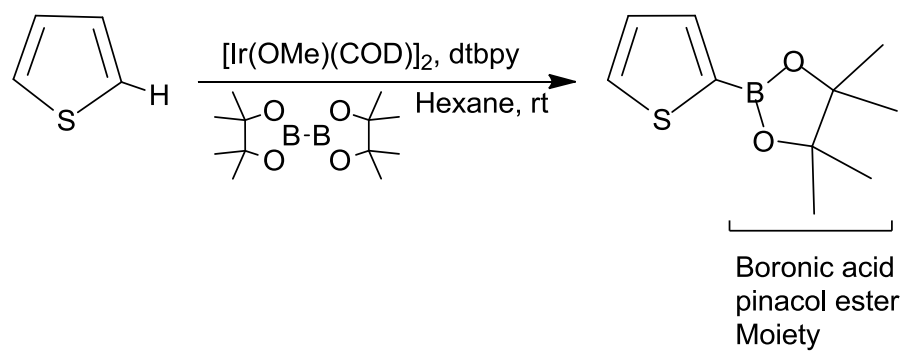

Scheme 2.4 Iridum direct C-H borylation reaction scheme.

Consideration has also been given to the boronic acid source for our borylation reactions. We have found pinacol boronic ester to be best suited for our purposes, as this class of boronate is known for its air stability and compatibility for silica based chromatography. Furthermore, the methyl groups on the pinacol boronic ester aid in the solubility of said compounds in common organic solvents. ${ }^{37}$

\subsubsection{One Pot Double Suzuki}

Continuing with the theme of reducing reaction steps involved in the synthesis of oligothiophene, we have sought to explore the viability of a one pot double Suzuki reaction. A one pot double Suzuki involves a dihalide arene group that undergoes consecutive Suzuki coupling reactions with two equivalents of a boronate species. ${ }^{38}$ In this way, we can cut down on the number of separate steps involved in oligothiophene elongation, while also circumventing the production of less soluble oligothiphenes, as the successive addition of the end-capping verdazyls increase solubility of the final product. One pot double Suzuki reactions have already been in use with non-radical syntheses for 
some time; we suspect that the use of this synthetic method will be just as successful as single Suzuki reactions involving verdazyls, but its confirmation will be another useful tool in the synthesis of verdazyl-organic semiconductor systems.

\subsection{Characterization of Thiophene-Verdazyl species}

Our planned synthesis route will yield a number of novel molecules in addition to our final target molecule, and we will use a range of characterization method for the study and identification of these compounds. For non-radical species, we will utilize proton and carbon NMR to decipher the molecules structure and connectivity, and corroborate those findings with molecular weight and fragmentation information provided by mass spectrometry (GC-MS or LC/HPLC-MS). Infrared spectrometry on the other hand will assist in identifying key functional groups of interest on our products.

Radical species will also be characterized through mass spectrometry and IR, but radicals typically do not yield useful information through NMR, as the magnetism of the radicals severely broadens the spectral linewidth, rendering the spectra broad and featureless. Instead we will utilize ESR spectrometry, which not only verifies the molecule as a species with unpaired electrons, it also provides information about the behavior of the unpaired electron and its interactions with neighboring atoms and unpaired electrons. UV-VIS spectrometry can indicate changes in the electronic structure of molecules, especially when compared to preceding species. If crystalline samples are obtained, X-ray crystallography can give a three dimensional structure and the intermolecular packing of analyzed compounds, the latter of which can be rather significant for physical properties that involve intermolecular interaction, such as 
conductivity. Finally, the characterization of both radical and non-radical species is rounded out with melting point measurements.

\subsection{Magnetism and Conductivity in Thiophene-Verdazyl species}

After the structural characterizations of novel compounds, we will begin to study the materials for interesting physical properties. We will carry out magnetic susceptibility measurements with a vibrating sample magnetometer, to determine the strength and type of magnetism in radical containing species, especially for our target diradical. Conductivity will be gauged through four-point conductivity measurements, this technique may also be used for certain magnetoresistance measurements under applied

magnetic field. ${ }^{39}$ Depending on the above results we also hope to assess the spin transport capabilities of our synthesized materials. The traditional approach would consist of a thin layer of our synthesized substance placed between two ferromagnetic electrodes. Spin polarized current is then injected through one electrode, passes through our substance and is measured at the second FM electrode. The second method for studying the spin polarizing and transporting properties of our diradicals, is by directly applying a magnetic field to a cell that contain our bisverdazyl sandwiched between two conductive nonmagnetic contacts. Through these experiments we hope to assess viability of the diradical(s) for spintronics applications. 


\section{Chapter 3: EXPERIMENTAL}

\subsection{General Methods}

All NMR samples were run in $\mathrm{CDCl}_{3}$ solvent (except for $\mathrm{H}-\mathrm{NMR}$ and C-NMR of 2,4diisopropyl-6-thiophene-5-boronic acid pinacol ester-1,2,4,5-tetrazane-3-one (4'), which were run in benzene-d6), and spectra were recorded on a Bruker instrument at $300 \mathrm{MHz}$, using TMS as reference. NMR spectra only encompass non-radical products. Mass spectroscopy for low molecular weight products was obtained with Agilent 5975B GCMS instrument with electron impact ionization, while higher molecular weight products (diradicals) were obtained with an Agilent 6520 Quadrupole TOF LC/MS instrument with electrospray ionization. ESR measurements were carried out with an X-Band spectrometer. Cyclic voltammetry runs were conducted in dichloromethane with tetrabutyl ammonium hexafluorophosphate as electrolyte at $0.2 \mathrm{~V} / \mathrm{s}$ scan rate. Ferrocene/ferriciniym was used as an internal reference, with an Au working electrode and $\mathrm{Ag} / \mathrm{AgCl}$ pseudo reference electrode. XRD data for radical species was obtained by Dr. Bruce Noll, Bruker AXS, and 3D structures were generated using the program Mercury. ${ }^{49}$ Melting point of novel compounds was measured with closed capillary tubes and Mel-Temp instrument. Reactions requiring air-free conditions (Suzuki and borylation reactions) were carried out using a dual manifold vacuum/nitrogen Schlenk line. Experimental spectra not presented in text are provided in the appendices.

Reaction steps involving the preparation of previously characterized starting materials for novel reaction steps were replicated from procedures outlined in previous papers. These include the synthesis of 5,5'-diiodo-2,2'-bithiophene from 2,2'-bithiophene and two 
equivalents of $\mathrm{N}$-iodosuccinimide, per procedure detailed in the supplementary information section of the Hwang et al paper, ${ }^{40}$ as well as all steps leading up to the formation of 2,4-diisopropylcarbonohydrazode bis-hydrochloride (1) from tert-butyl 2isopropylhydrazinecarboxylate, which follow the procedure outlined in the Pare et al. paper. ${ }^{31}$ Detailed procedures for novel reaction steps are as follows.

\subsection{Thiophene-Verdazyl}

\subsubsection{Preparation of 2,4-Diisopropyl-6-thiophene-1,2,4,5-tetrazane-3-one(3)}

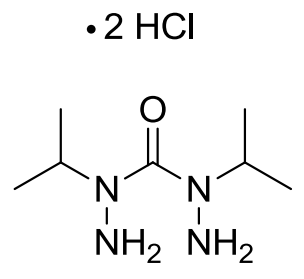

1

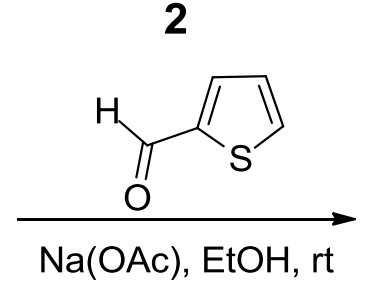

$\mathrm{Na}(\mathrm{OAc}), \mathrm{EtOH}, \mathrm{rt}$

Scheme 3.1 Synthesis of compound (3).

A $250 \mathrm{ml}$ Erlenmeyer flask was charged with $4.13 \mathrm{~g}(17.0 \mathrm{mmol})$ of 2,4-diisopropyl carbonobishydrazide dihydrochloride $\mathbf{1}$, along with $1.98 \mathrm{~g}$ (17.0 mmol) of thiophenecarboxaldehyde 2 , and dissolved in the least amount of ethanol ( $\sim 50 \mathrm{ml})$. Next, $2.8 \mathrm{~g}(34.0$ $\mathrm{mmol}$ ) of sodium acetate was added to the flask along with additional ethanol (70ml) and mixed with a magnetic stirbar for several minutes until complete dissolution. The solution was then allowed to stand in the hood overnight for 24 hours. The following day some precipitate had formed, which was removed by vacuum filtration and the solvent was removed from the filtrate using a rotary evaporator. The remaining crude solid was recrystallized from heptane to obtain $2.68 \mathrm{~g} \mathrm{(10} \mathrm{mmol,} \mathrm{59 \% ,)} \mathrm{of} \mathrm{pale} \mathrm{yellow} \mathrm{tetrazane} 3$. 
Mp (capillary) 150-153 ${ }^{\circ} \mathrm{C} . \mathrm{IR}\left(\mathrm{cm}^{-1}\right) 3208(\mathrm{~N}-\mathrm{H}), 2967(\mathrm{C}-\mathrm{H}), 1579$ (C=O). H-NMR $(300 \mathrm{MHz}) 1.15(\mathrm{~d}, 6 \mathrm{H}, J=6.0 \mathrm{~Hz}), 1.17(\mathrm{~d}, 6 \mathrm{H}, J=6.0 \mathrm{~Hz}), 3.88(\mathrm{~d}, 2 \mathrm{H} J=12.0 \mathrm{~Hz})$, 4.68 (septet, $2 \mathrm{H} J=6.0 \mathrm{~Hz}), 4.76(\mathrm{~s}, 1 \mathrm{H}), 7.06(\mathrm{dd}, 1 \mathrm{H}, J=6.0,3.0 \mathrm{~Hz}), 7.21(\mathrm{~d}, 1 \mathrm{H}, J=$ $6.0 \mathrm{~Hz}), 7.34$ (d, 1H, $J=6.0 \mathrm{~Hz})$. C-NMR (300 MHZ) 18.39, 19.49, 47.77, 69.16, 125.31, 126.08, 127.14, 138.15. MS for $\mathrm{C}_{12} \mathrm{H}_{20} \mathrm{~N}_{4} \mathrm{O}_{1} \mathrm{~S}_{1}$ calculated as 268, found as (GC-MS, electron ionization) $\mathrm{M}^{+}=268 \mathrm{~m} / \mathrm{z}$. MS fragmentation relative abundances in $\mathrm{m} / \mathrm{z}, 268$ (20), 220 (100), 192 (50), 153 (51), 147 (23), 110 (95).

\subsubsection{Oxidation to 1,5-Diisopropyl-3-thiophene-6-oxoverdazyl (4)}
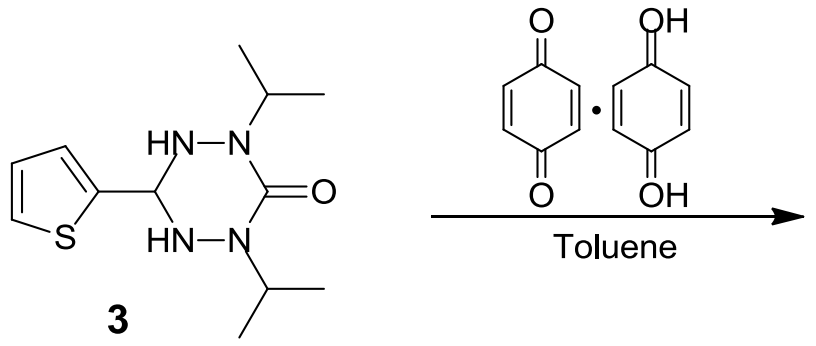

Scheme 3.2 Synthesis of compound (4).

$2.68 \mathrm{~g}$ (10 mmol) of 2,4-diisopropyl-6-thiophene-1,2,4,5-tetrazane-3-one 3 was added to a $500 \mathrm{ml}$ round-bottom flask, along with $3.29 \mathrm{~g}(15 \mathrm{mmol})$ of quinhydrone and a magnetic stirbar. Next, $350 \mathrm{ml}$ of toluene was added to the round-bottom and a reflux apparatus was setup with a condenser over a heated oil bath. After refluxing for an hour, the solution had changed color from pale yellow to a deep red. The reaction progress was monitored through TLC comparison of the starting tetrazane and reaction solution; the disappearance of the tetrazane spot from the reacting solution spot indicated reaction completion. The round bottom flask and its contents were allowed to cool to room temperature over 1.5 hours. At this point some hydroquinone had crystalized out of the 
solution. To maximize the removal of the hydroquinone, the flask was placed in a freezer for an hour to force more hydroquinone out of solution. The chilled slurry was then vacuum filtered and washed with cold toluene. The filtrate solution was evaporated under a high vacuum rotary evaporator to give the crude product as a cherry red solid. Product 4 was purified through several washes with cold hexane, vacuum filtration, and subsequent evaporation of the filtrate to give $1.62 \mathrm{~g}(6.1 \mathrm{mmol})$ of red verdazyl 4 crystal with $61 \%$ yield. Mp (capillary) 57.0-60.0 ${ }^{\circ} \mathrm{C}$. ESR run carried out in degassed hexane, with $\mathrm{g}=$ 2.003, aN $=6 \mathrm{G}$ as parameters. IR $\left(\mathrm{cm}^{-1}\right), 2987(\mathrm{C}-\mathrm{H}), 1675(\mathrm{C}=\mathrm{O})$. UV-VIS (nm) $\lambda \max _{1}$ $=426\left(\varepsilon_{1}=1.2 \times 10^{3} \mathrm{~L} / \mathrm{mol} . \mathrm{cm}\right), \lambda \max _{2}=514\left(\boldsymbol{\varepsilon}_{2}=4 \times 10^{2} \mathrm{~L} / \mathrm{mol} . \mathrm{cm}\right) . \mathrm{MS}$ for $\mathrm{C}_{12} \mathrm{H}_{17} \mathrm{~N}_{4} \mathrm{OS}$ calculated as 265 , found as (GC-MS, electron ionization) $\mathrm{M}^{+}=265 \mathrm{~m} / \mathrm{z}$. MS fragmentation relative abundances in m/z, 265 (35), 223 (19), 181 (100), 110 (90).

\subsection{Iridium Borylation of Thiophene-Verdazyl}

\subsubsection{Synthesis of 1,5-Diisopropyl-3-thiophene-5-boronic acid pinacol ester-6- oxoverdazyl (5)}
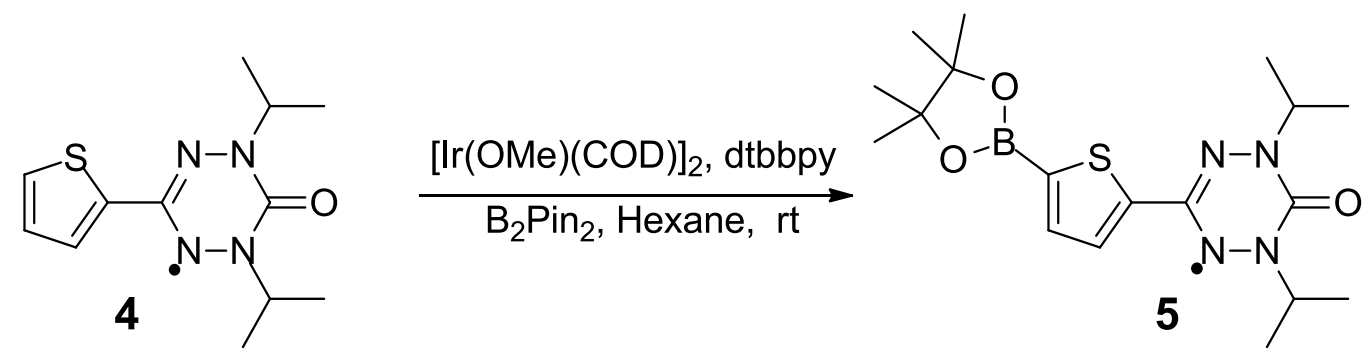

Scheme 3.3 Synthesis of compound (5).

The iridium catalyst $\operatorname{Ir}(\mathrm{OMe})[\mathrm{COD}]_{2}$ was prepared according to the procedure described in the supplementary information section of the Tajuddin et al paper, ${ }^{41}$ while the procedure for iridium catalyzed direct C-H borylation of 1,5-Diisopropyl-3- 
thiophene-6-oxoverdazyl 4 is a variation on the procedure described in the Ishiyama et al paper. $^{42}$

A two-neck $500 \mathrm{ml}$ round-bottom flask was charged with catalytic amounts of $[\mathrm{Ir}(\mathrm{OMe})(\mathrm{COD})]_{2}(0.02 \mathrm{mmol}, 0.013 \mathrm{~g})$ and its ligand, dtbpy (0.04 mmol, 0.011g), along with a magnetic stirbar. The round-bottom was then fitted with a septum on the side neck and the middle neck was attached to a condenser connected to a duel manifold vacuum/nitrogen Schlenk line. The round bottom was subsequently degassed and $0.64 \mathrm{~g}$ ( $2.5 \mathrm{mmol})$ of the boronate source, bis(pinacolato) diboron was added to the flask, followed by a second degassing. In a separate pear shaped flask, $20 \mathrm{ml}$ of hexane was added and the flask was covered with a septum. One side of the septum was punctured with a needle connected to a nitrogen line, while a second open ended needle was placed onto the other side of the septum, enabling the purging of hexane over a 5-minute duration. While the first needle continues to pump the flask with nitrogen, the second needle was removed and attached to a syringe. The syringe needle was then inserted through the septum of the pear-shaped flask without touching the solvent and the syringe was filled and subsequently emptied of nitrogen multiple times before finally filling the syringe with the degassed solvent and immediately transferring the content to the first two necked round-bottom still under nitrogen. After further degassing of the round bottom, the mix was stirred and the flask lowered into an oil bath and heated at $50{ }^{\circ} \mathrm{C}$ for 10 minutes.

The catalyst was fully primed once the solution turned a dark red color. Meanwhile in the previously used pear shaped flask $1.19 \mathrm{~g}$ (4.5 mmol, ) of 1,5-Diisopropyl-3-thiophene- 
6-oxoverdazyl 4 was added along with an additional $200 \mathrm{ml}$ of hexane and the mix was stirred, degassed, and transferred to the main flask. After a final degassing, the two-neck round-bottom was lowered into the oil bath and allowed to stir at $50{ }^{\circ} \mathrm{C}$ for 24 hours.

Once the reaction was completed, the solution was left to cool and subsequently extracted with hexane and water in a separatory funnel. The resulting extracted solution was dried over anhydrous magnesium sulfate, gravity filtered and the solvent evaporated using a rotary evaporator, giving a dark red crude product

Purification was done through silica gel column chromatography, and eluted with 2:3 dichloromethane-hexane solvent, followed by recrystallization with water/ethanol, giving $0.25 \mathrm{~g}(0.64 \mathrm{mmol}$ ) of compound 5 as fine red crystals, with a yield of $14.2 \%$. Mp (capillary) $121-133{ }^{\circ} \mathrm{C}$. ESR run was carried out in degassed hexane, with $\mathrm{g}=2.0040$ and $\mathrm{aN}=6 \mathrm{G}$ as parameters. IR $\left(\mathrm{cm}^{-1}\right), 2977(\mathrm{C}-\mathrm{H}), 1681(\mathrm{C}=\mathrm{O})$. UV-VIS $(\mathrm{nm}), \lambda \max _{1}$ $430\left(\boldsymbol{\varepsilon}_{1}=1.3 \times 10^{3} \mathrm{~L} / \mathrm{mol} . \mathrm{cm}\right), \lambda \max _{2} 520\left(\boldsymbol{\varepsilon}_{2}=4.6 \times 10^{2} \mathrm{~L} / \mathrm{mol} . \mathrm{cm}\right)$. MS for $\mathrm{C}_{18} \mathrm{H}_{28} \mathrm{BN}_{4} \mathrm{O}_{3} \mathrm{~S}$ calculated as 391, found as (GC-MS, electron ionization) $\mathrm{M}^{+}=391 \mathrm{~m} / \mathrm{z}$. MS fragmentation relative abundances, 391 (63), 349 (18), 307 (100), 236 (50), 207 (34), 177 (18), 154 (22), 136 (70), 110 (17), 83 (24), 56 (37). 


\subsection{Alternative Route for Borylated-Thiophene-Verdazyl}

\subsubsection{Synthesis of 5-Formyl-2-thienylboronic acid pinacol ester (3')}
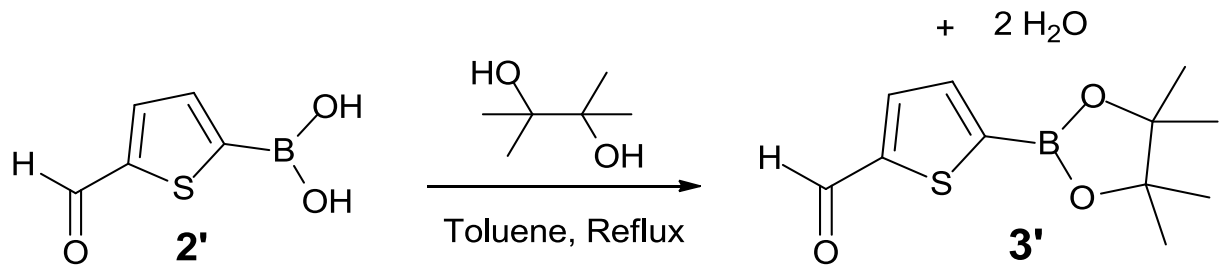

Scheme 3.4 Synthesis of compound (3').

To a 1L round-bottom flask $5.33 \mathrm{~g}$ (34.2 mmol) of 5-Formyl-2-thienylboronic acid 2', and excess pinacol $(43.0 \mathrm{mmol}, 5.10 \mathrm{~g})$ were added. Next, $750 \mathrm{ml}$ of toluene and a magnetic stirbar were added to the same round-bottom, and the flask was equipped with a Dean-Stark/condenser apparatus and lowered into a heated oil bath at $120^{\circ} \mathrm{C}$. The reaction mix was refluxed overnight, while the Dean-stark apparatus collected the water side product. The next day, the round-bottom was removed from heating and cooled to room temperature. The flask content was dried with anhydrous sodium sulfate and gravity filtered. The filtrate solution was evaporated with a rotary evaporator, giving brown colored crude solid. Product 3' was purified through recrystallization with heptane to give light brown crystals $(21.0 \mathrm{mmol}, 5.0 \mathrm{~g})$ with a yield of $61.5 \%$. H-NMR (300 MHz), 1.37 (s, 12H), 7.67 (d, 1H, $J=3 \mathrm{~Hz}), 7.82(\mathrm{~d}, 1 \mathrm{H}, J=3 \mathrm{~Hz}), 9.99$ (s, 1H). MS for $\mathrm{C}_{11} \mathrm{H}_{15} \mathrm{BO}_{3} \mathrm{~S}$ calculated as 238 , found as (GC-MS, electron ionization) $\mathrm{M}^{+}=238 \mathrm{~m} / \mathrm{z}$. MS fragmentation relative abundances, 238 (80), 223 (96), 154 (69), 139 (100). 


\subsubsection{Synthesis of 2,4-Diisopropyl-6-thiophene-5-boronic acid pinacol ester-1,2,4,5- tetrazane-3-one (4')}

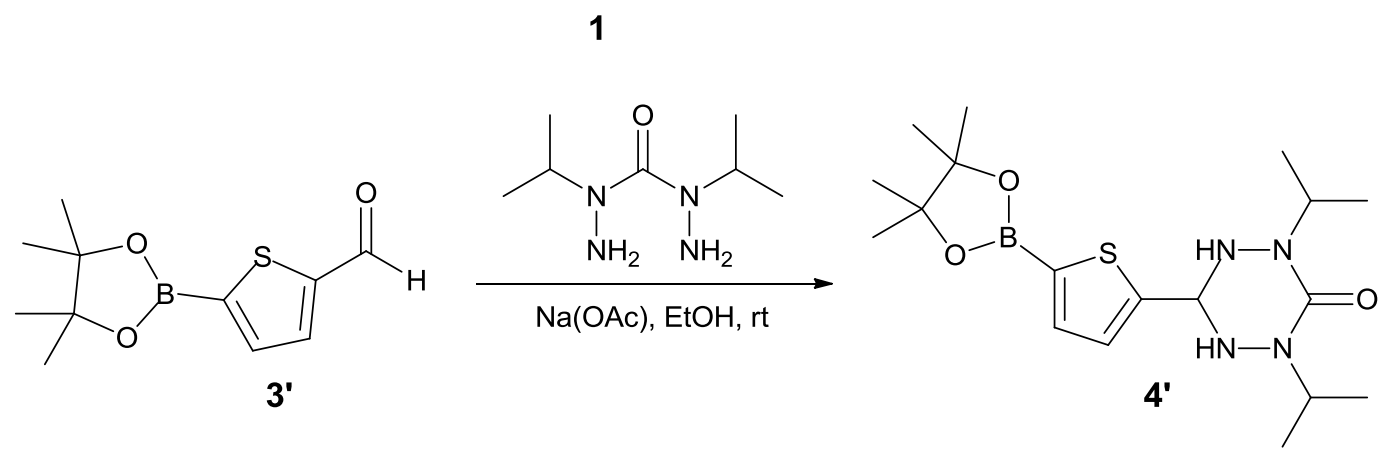

Scheme 3.5 Synthesis of compound (4').

A $500 \mathrm{ml}$ Erlenmeyer flask was charged with $4.65 \mathrm{~g}(19.5 \mathrm{mmol})$ of 5-Formyl-2thienylboronic acid pinacol ester $\mathbf{3}^{\prime}$, and $4.80 \mathrm{~g}$ (19.5 mmol) of bishydrazide 1. A magnetic stirbar and $70 \mathrm{ml}$ of ethanol were added to the above flask, and the content were mixed until all solids were dissolved. Next, $3.21 \mathrm{~g} \mathrm{(39.1} \mathrm{mmol)} \mathrm{of} \mathrm{sodium} \mathrm{acetate,} \mathrm{along}$ with an additional $350 \mathrm{ml}$ of ethanol were added to the reaction flask, and the contents were stirred for an hour. After the mixing the solution was allowed to stand in the hood for 24 hours. The next day some precipitate had formed in the flask, this precipitate was removed through vacuum filtration and the remaining solvent was removed with a rotary evaporator, giving a pale-yellow crude product. Recrystallization from heptane gave 3.94 $\mathrm{g}(10 \mathrm{mmol})$ of tetrazane $4^{\prime}$ with a yield of $51 \%$. Mp (capillary) $196-203{ }^{\circ} \mathrm{C}$. IR $\left(\mathrm{cm}^{-1}\right)$ $3230(\mathrm{~N}-\mathrm{H}), 2975(\mathrm{C}-\mathrm{H}), 1581(\mathrm{C}=\mathrm{O})$. H-NMR (300 MHz), 1.15 (d, 6H, $J=3 \mathrm{~Hz}), 1.17$ (d, $6 \mathrm{H}, J=3 \mathrm{~Hz}), 1.36(\mathrm{~s}, 12 \mathrm{H}), 3.90(\mathrm{~d}, 2 \mathrm{H}, J=12 \mathrm{~Hz}), 4.67$ (septet, 2H, $J=6 \mathrm{~Hz}), 4.74$ (t, 1H, $J=12 \mathrm{~Hz}), 7.29(\mathrm{~d}, 1 \mathrm{H}, J=3 \mathrm{~Hz}), 7.57(\mathrm{~d}, 1 \mathrm{H}, J=3 \mathrm{~Hz}) . \mathrm{C}-\mathrm{NMR}(300 \mathrm{MHz})$, $18.09,19.36,24.51,47.52,69.13,83.87,126.61,126.70,137.28,145.96,153.62$. MS for 
$\mathrm{C}_{18} \mathrm{H}_{32} \mathrm{BN}_{4} \mathrm{O}_{3} \mathrm{~S}$ calculated as 394, found as (GC-MS, electron ionization) $\mathrm{M}^{+}=394 \mathrm{~m} / \mathrm{z}$. MS fragmentation relative abundances, 394 (41), 279 (42), 236 (100), 207 (42), 56 (42).

\subsubsection{Oxidation to 1,5-Diisopropyl-3-thiophene-5-boronic acid pinacol ester-6- oxoverdazayl (5)}
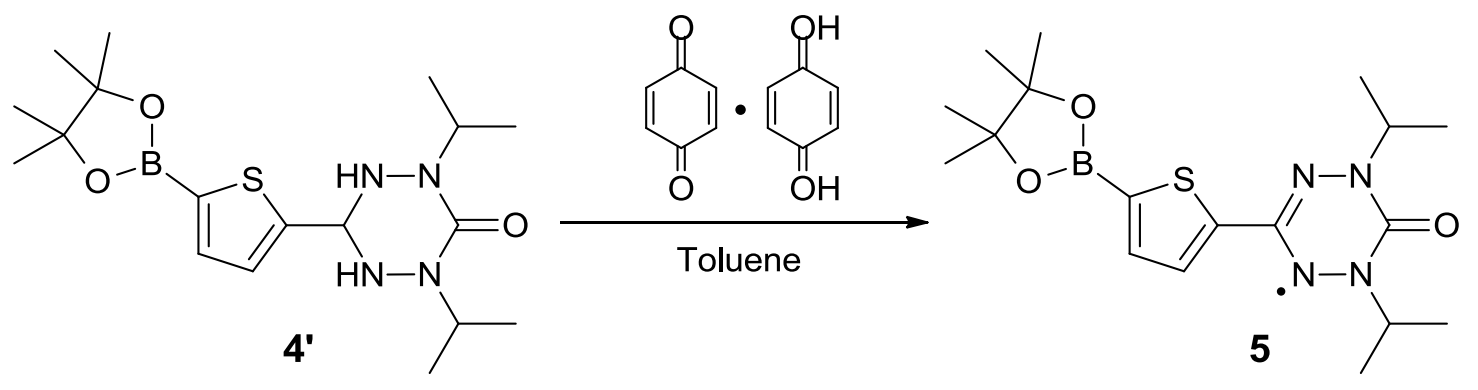

Scheme 3.6 Alternative synthesis of compound (5).

$3.68 \mathrm{~g} \mathrm{(9.3} \mathrm{mmol)} \mathrm{of} \mathrm{2,4-Diisopropyl-6-thiophene-5-boronic} \mathrm{acid} \mathrm{pinacol} \mathrm{ester-}$

1,2,4,5-tetrazane-3-one 4', $3.1 \mathrm{~g}$ (14.1 mmol) of quinhydrone and a magnetic sitrbar were added to a $500 \mathrm{ml}$ round-bottom flask. Next, $400 \mathrm{ml}$ of toluene were added to the roundbottom and a reflux was setup by fitting the round-bottom with a condenser and lowering the flask into a heated oil bath. After an hour of reflux, the reaction solution had become dark red colored and the completion of the reaction was assessed through TLC. Once complete, the reaction solution was allowed to cool to room temperature over 1.5 hours, then placed in a freezer for an additional hour. The cold slurry was vacuum filtered and washed with cold toluene to remove most the hydroquinone byproduct. The resulting filtrate solvent was removed with a high vacuum rotary evaporator to give crude red solid. Product 5 was purified with several cold hexane washes, gravity filtration, and evaporation with a rotary evaporator to conclude the alternative synthesis with $2.72 \mathrm{~g}$ (6.96 mmol) of verdazyl 5 and $49 \%$ yield. Mp (capillary) $121-133{ }^{\circ} \mathrm{C}$. ESR run in 
degassed hexane. IR cm $\mathrm{cm}^{-1} 2977(\mathrm{C}-\mathrm{H}), 1681(\mathrm{C}=\mathrm{O})$. UV-VIS (nm), $\lambda \max _{1}(430), \lambda \max _{2}$ (520). MS for $\mathrm{C}_{18} \mathrm{H}_{29} \mathrm{BN}_{4} \mathrm{O}_{3} \mathrm{~S}$ calculated as 391, found as (GC-MS, electron ionization) $\mathrm{M}^{+}=391 \mathrm{~m} / \mathrm{z}$. MS fragmentation relative abundances, 391 (63), 349 (18), 307 (100), 236 (50), 207 (34), 177 (18), 154 (22), 136 (70), 110 (17), 83 (24), 56 (37).

\subsection{Double Suzuki}

\subsubsection{Synthesis of Bis (1, 5-diisopropyl-6-oxoverdazyl)-3,3'- $\alpha$-quaterthiophene (6)}

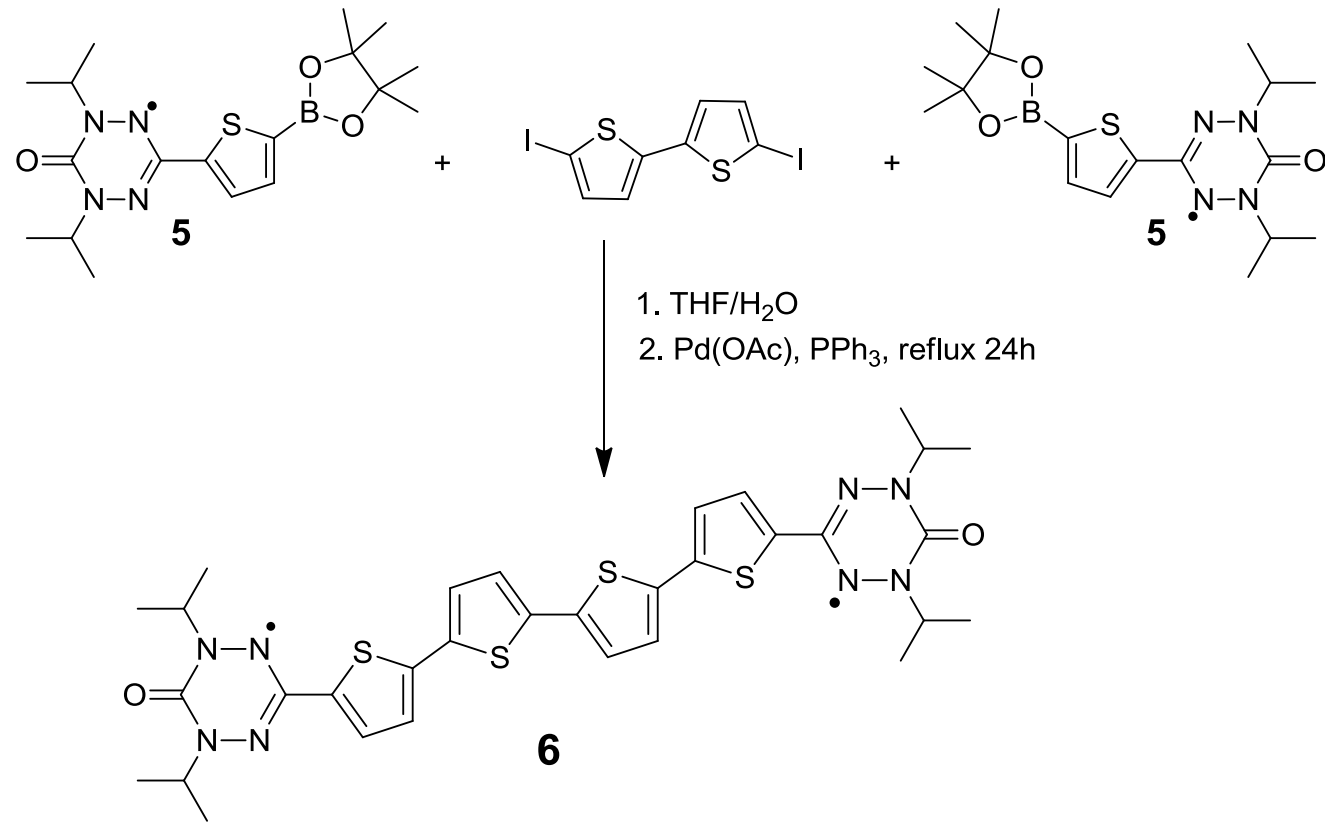

Scheme 3.7 Synthesis of compound (6).

One equivalent of 5,5'-diiodo-2,2'-bithiophene (0.38 mmol, $0.15 \mathrm{~g})$ and two equivalents of 1,5-Diisopropyl-3-thiophene-5-boronic acid pinacol ester-6-oxoverdazayl $5(0.82 \mathrm{mmol}, 0.32 \mathrm{~g})$, were added to a $100 \mathrm{ml}$ two neck round-bottom flask, along with catalytic amounts of palladium catalyst $\mathrm{Pd}(\mathrm{OAc})_{2}(0.053 \mathrm{mmol}, 0.012 \mathrm{~g})$, its ligand $\mathrm{PPh}_{3}$ (0.17 mmol, $0.045 \mathrm{~g})$, and a stirbar. The side neck of the round-bottom was fitted with a septum, while the main neck was attached to a condenser connected to a dual manifold 
vacuum/nitrogen Schlenk line. The round-bottom and its content were degassed with multiple vacuum/nitrogen pump cycles.

In a separate $100 \mathrm{ml}$ pear-shaped flask fitted with a septum, $50 \mathrm{ml}$ of THF was degassed through purging with nitrogen and vented with a second needle for 5 minutes. While the solvent was still being pumped with nitrogen, the THF was taken up with a previously vented syringe and the solvent quickly transferred to the main round-bottom, and the reaction mixture was subsequently degassed once more while stirring. In the previously used pear shaped flask, $0.20 \mathrm{~g}(1.5 \mathrm{mmol})$ of $\mathrm{K}_{2} \mathrm{CO}_{3}$ was added along with 20 $\mathrm{ml}$ of deionized water. The flask was gently swirled until the base was completely dissolved, then purged with nitrogen. After degassing, the solution was carefully transferred to the main round-bottom flask with a degassed syringe. The content in the round-bottom was degassed a final time while stirring, the flask was then lowered into an oil bath and refluxed for $24 \mathrm{~h}$, while still under a nitrogen flow.

The following day the reaction flask was removed from heating and allowed to cool to room temperature. Next, the reaction solution underwent extraction with water and dichloromethane in a separatory funnel, and the resulting organic layer was evaporated with a rotary evaporator, giving a dark colored solid. Bisverdazyl 6 was isolated by silica gel column chromatography with 2:3 hexane: dichloromethane eluting solution, and subsequently recrystallized with heptane. The purified product $6(0.24 \mathrm{mmol}, 0.17 \mathrm{~g})$ was a dark gray powder with a yellow tint, and a yield of $63 \%$. Mp (capillary) $172-174{ }^{\circ} \mathrm{C}$. ESR run was carried out in degassed dichloromethane, with $\mathrm{g}=2.0040, \mathrm{aN}=3.2 \mathrm{G}, \mathrm{aN}=$ 2.64 $\mathrm{G}, \mathrm{aH}=0.89 \mathrm{G}$ and linewidth $=0.7 \mathrm{G}$ as parameters. IR $\left(\mathrm{cm}^{-1}\right), 2983(\mathrm{C}-\mathrm{H}), 1677$ 
$(\mathrm{C}=\mathrm{O}) . \mathrm{UV}-\mathrm{VIS}(\mathrm{nm}), \lambda \max _{1}=428\left(\boldsymbol{\varepsilon}_{1}=5.5 \times 10^{4} \mathrm{~L} / \mathrm{mol}_{\mathrm{cm}}\right), \lambda \max _{2}=592\left(\boldsymbol{\varepsilon}_{2}=7.7 \times 10^{2}\right.$

$\mathrm{L} / \mathrm{mol} . \mathrm{cm}$ ). MS for $\mathrm{C}_{32} \mathrm{H}_{36} \mathrm{~N}_{8} \mathrm{O}_{2} \mathrm{~S}_{4}$ calculated as 692 , found as (LC-MS, electrospray) $\mathrm{M}^{+}$ $=695\left(692+3 \mathrm{H}^{+}\right)$. Magnetic Susceptibility C $($Curie Constant $)=0.68 \mathrm{~cm}^{3} \cdot \mathrm{K} / \mathrm{mol}$.

\subsubsection{Synthesis of Bis (1, 5-diisopropyl-6-oxoverdazyl)-3,3'-biphenyl-4,4'- bithiophene (7)}

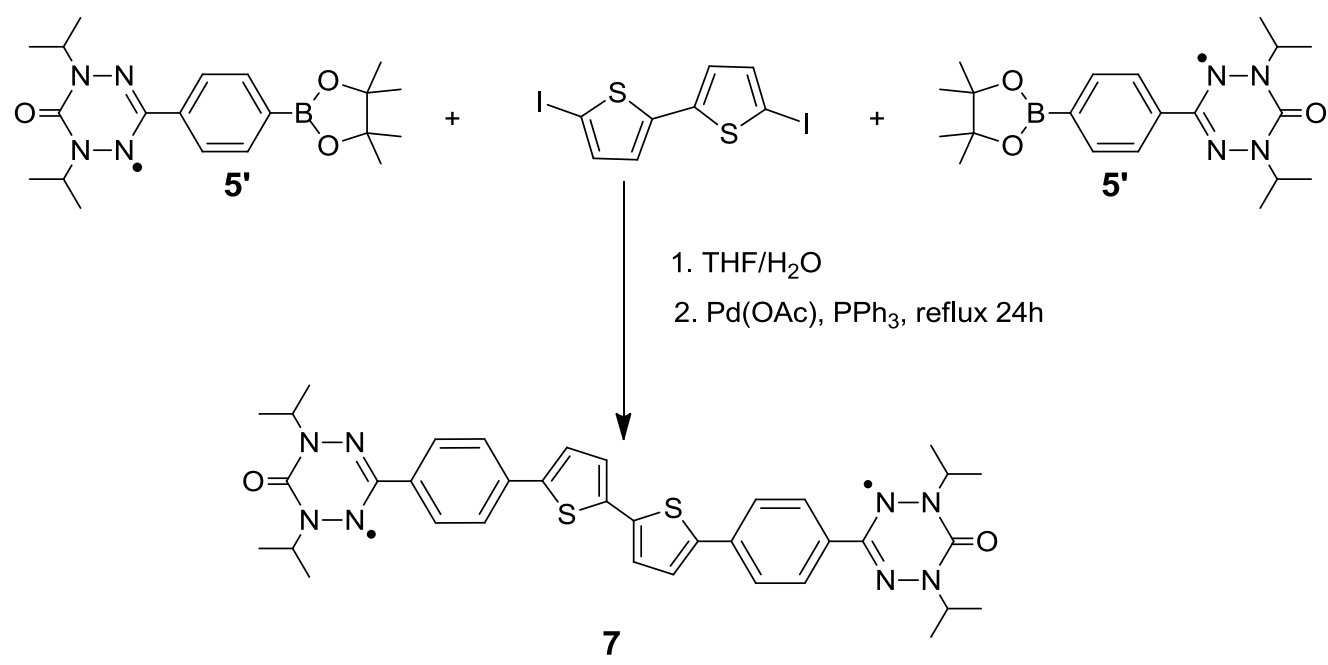

Scheme 3.8 Synthesis of compound (7).

One equivalent 5,5'-diiodo-2,2'-bithiophene ( $0.036 \mathrm{mmol}, 0.015 \mathrm{~g})$ and two equivalents of 1,5-Diisopropyl-3-Phenyl-4-boronic acid pinacol ester-6-oxoverdazayl 5' $(0.070 \mathrm{mmol}, 0.027 \mathrm{~g})$ were added to a $50 \mathrm{ml}$ two neck round-bottom flask, along with catalytic amounts of palladium catalyst $\mathrm{Pd}(\mathrm{OAc})_{2}(0.0045 \mathrm{mmol}, 0.001 \mathrm{~g})$, its ligand $\mathrm{PPh}_{3}$ $(0.011 \mathrm{mmol}, 0.003 \mathrm{~g})$, and a stirbar. The side neck of the round-bottom was fitted with a septum, while the main neck was attached to a condenser connected to a dual manifold vacuum/nitrogen schlenk line. The round-bottom and its content were degassed with multiple vacuum/nitrogen pump cycles. 
In a separate $50 \mathrm{ml}$ pear-shaped flask fitted with a septum, $20 \mathrm{ml}$ of THF was degassed through purging with nitrogen for 5 minutes. While the pear-shaped flask was still under nitrogen flow, the solvent was quickly transferred to the main round-bottom with a previously degassed syringe, and the reaction mixture was subsequently degassed once more while stirring. In the previously used pear shaped flask $0.027 \mathrm{~g}(0.19 \mathrm{mmol})$ of $\mathrm{K}_{2} \mathrm{CO}_{3}$ was added along with $10 \mathrm{ml}$ of deionized water. The flask was gently swirled until the base was completely dissolved, this was followed by degassing of the aqueous solution through purging with nitrogen. After degassing, the solution was carefully transferred to the main round-bottom flask with a degassed syringe. The content in the round-bottom was degassed a final time while stirring, and the flask was then lowered into an oil bath and refluxed for $24 \mathrm{~h}$.

The following day the reaction flask was removed from heating and allowed to cool to room temperature. The reaction solution underwent extraction with water and dichloromethane in a separatory funnel, and the resulting organic layer was dried with anhydrous magnesium sulfate, gravity filtered, and evaporated with a rotary evaporator, giving a crude orange solid. The product was further isolated by silica gel column chromatography with 2:3 hexane: dichloromethane elueting solution, and recrystallized with heptane, giving $0.010 \mathrm{~g}(0.015 \mathrm{mmol})$ of purified bisverdazyl 7 as an orange powder with a yield of $41 \%$. Mp (capillary) decomposition at $193-210{ }^{\circ} \mathrm{C}$ range. ESR run carried out in degassed xylene, with $\mathrm{g}=2.0040, \mathrm{aN}=3.23 \mathrm{G}, \mathrm{aN}=2.54 \mathrm{G}, \mathrm{aH}=0.78 \mathrm{G}$ and linewidth $=0.9 \mathrm{G}$ as parameters. IR $\left(\mathrm{cm}^{-1}\right), 2983(\mathrm{C}-\mathrm{H}), 1668(\mathrm{C}=\mathrm{O}) . \mathrm{UV}-\mathrm{VIS}(\mathrm{nm})$ 
$\lambda \max _{1}=264, \lambda \max _{2}=398$. MS for $\mathrm{C}_{36} \mathrm{H}_{40} \mathrm{~N}_{8} \mathrm{O}_{2} \mathrm{~S}_{2}$ calculated as $680, \mathrm{M}^{+}$not obtained experimentally. Magnetic Susceptibility C (Curie constant) $=0.56 \mathrm{~cm}^{3} . \mathrm{K} / \mathrm{mol}$. 


\section{Chapter 4: RESULTS \& DISCUSSION}

\subsection{Failed Reactions \& Their Role in Designing Successful Reaction Routes}

Before delving into the results of our successful reactions, it would be helpful to first discuss some of the unsuccessful reactions over the course of our research. While these setbacks may not have yielded the desired products, the insights gained from these failures were key in designing the latter successful reaction routes.

For our initial approach to synthesizing oligothiophene-verdazyl systems, we sought to first synthesize the oligothiophene backbone, and only couple the thiophenes to the verdazyl precursor in the final steps. The primary reasoning behind this approach was to postponed the use of the verdazyl precursor, bishydrazide $\mathbf{1}$, which requires a multistep synthesis to obtain. The lengthy synthesis of bishydrazide $\mathbf{1}$ is a serious limiting factor; thus, the final product yield is likely to be maximized with a delayed incorporation of bishydrazide in the synthesis route. To this end, we sought to make carboxaldehyde endcapped quaterthiophene, through a double suzuki coupling reaction as seen in Scheme 4.1; the carboxaldehydes are of course necessary to form end capped tetrazanes, which can then be oxidized to verdazyls.

After several attempts at this reaction, with varying reaction conditions, we were unable to obtained the desired product. The failure of the above reaction can primarily be attributed to poor solubility. 5-formyl-2-thienylboronic acid was found to be poorly soluble in common non-polar organic solvents, likely because of its relative polarity of the boronic acid end of the compound. Non-polar Dibromo bithiophene, on the other hand, only dissolved in non-polar solvents. While both reagents do dissolve in non-polar 
solvents at high temperatures, if boronic acids are left in basic conditions for extended periods, especially at elevated temperatures as with Suzuki coupling reaction conditions, they are known to undergo protodeborylation (loss of boronic acid functional group). ${ }^{37}$ NMR and Mass Spectrometry results of the reaction mix indicated only the presence of 2thiophenecarboxaldehyde and 5,5'-dibromo-2,2'-bithiophene, the former of which is the deborylated product of our starting boronic acid, reinforcing the likeliness of protodeborylation.

While in retrospect it may seem self-evident that the poor solubility of lightly substituted or unsubstituted oligothiophenes would make them intractable for most reaction types, we did not initially realize the full extent of this limiting factor. Indeed, even if we had succeeded in synthesizing the carboxaldehyde end-capped quarterthiophene, the product would still be unwieldy for the follow up reaction, which would require dissolution in ethanol.

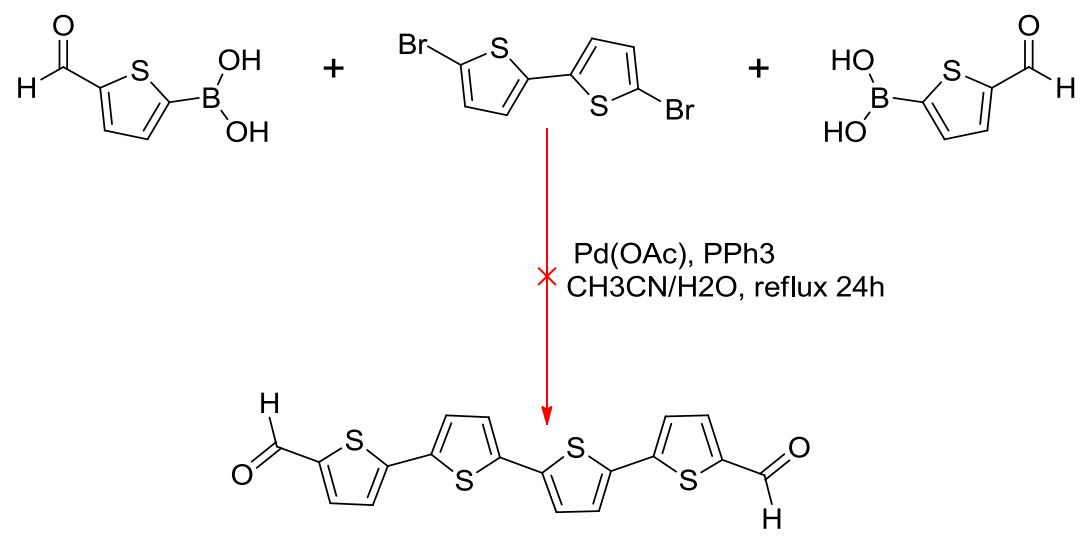

Scheme 4.1 Failed double suzuki coupling.

The unsuccessful reaction above led us to reconsider our synthesis strategy, and instead opt for growing the oligothiophene backbone from the verdazyl moiety. To do 
this, we coupled a single thiophene to the verdazyl precursor, which would give us our first novel molecule in the form of the thiophene-tetrazane characterized in the next section. This new approach alleviates the previously discussed solubility issues by equipping the prospective molecules with the isopropyl groups of the tetrazanes and verdazyls, which significantly enhance solubility for the entire molecule.

The second notable reaction hurdle concerned the borylation of thiophenes, specifically our attempt to form a di(boronate) of bithiophene from its dihalide. Previously the Brook research groups' approach to borylation of arenes was to carry out Miyaura borylation of halo-arenes. This approach was generally successful as it involved only the borylation of a mono halide, but we found the results less promising with dihalide borylation.

As seen in Scheme 4.2, the Miyaura borylation of one side of the dihalide can be followed up with unintended Suzuki homo/cross-coupling side reactions, between either the mono borylated product itself or the starting dihalide, yielding a number of undesired oligothiophenes of varying sizes. The reaction was replicated with different conditions, in the hopes that the Suzuki side reactions could be curbed, however, we were unsuccessful in this regard 


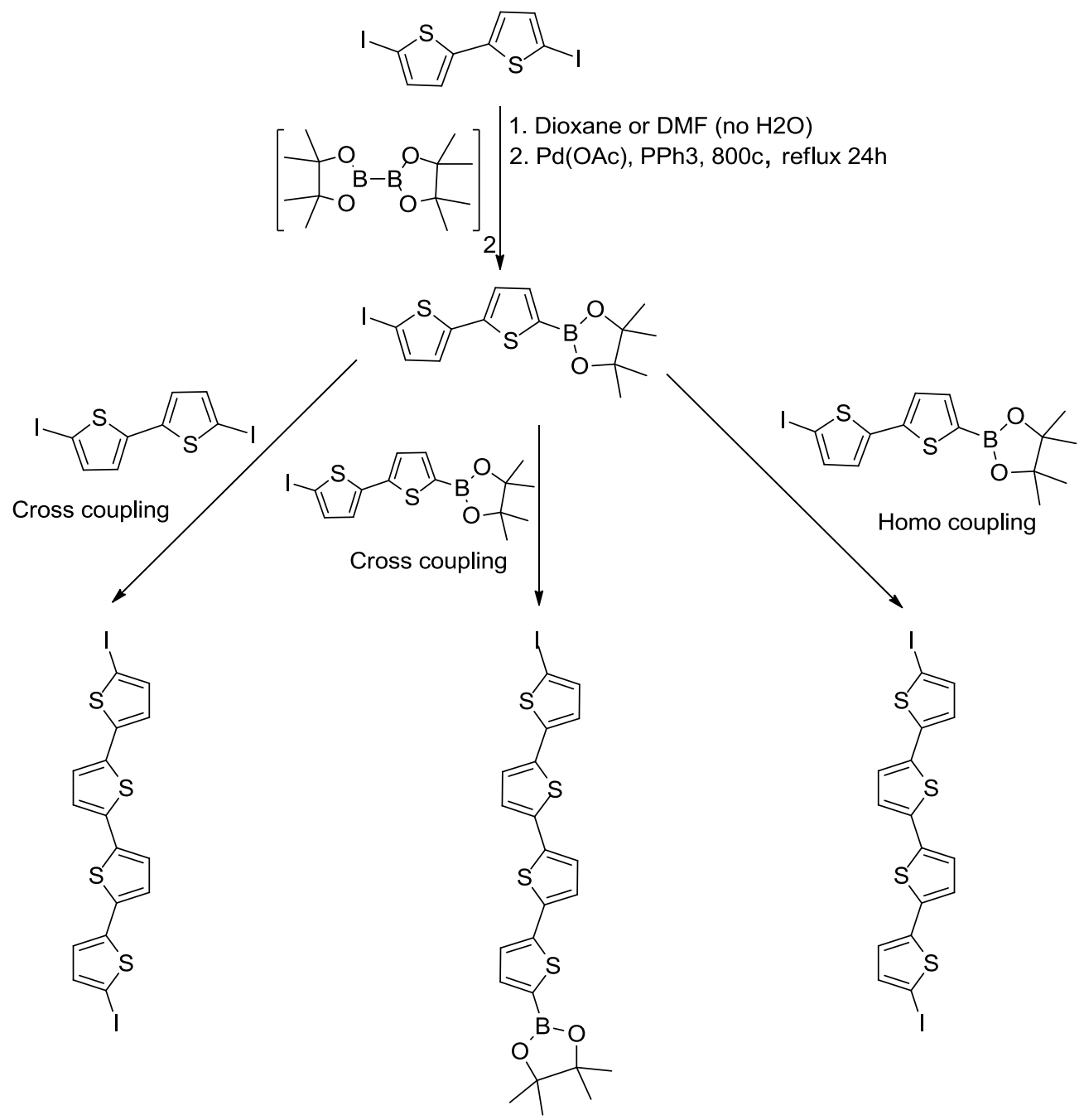

Scheme 4.2 Miyaura di(borylation) of dihalide.

This prompted a reassessment of our approach to thiophene borylation. Instead of borylating halides through Miyaura borylation, we could instead opt for direct C-H iridium catalyzed borylation of non-halogenated thiophenes, and thus avoid unwanted Suzuki side reactions. Furthermore, an earlier attempt at iodination of aryl-verdazyls demonstrated the incompatibility of the acidic iodination reaction conditions, which lead to the decomposition of the reacting verdazyl. Considering the above findings, we 
concluded that the best approach to obtaining our target molecules would be to borylate aryl-verdazyls through iridium catalyzed borylation, while non-radical aryl groups would be separately halogenated to provide the necessary reagents for a follow up Suzuki coupling reactions that would grow the thiophene chain from the verdazyl. This approach ultimately proved successful for synthesizing target molecules, demonstrating the role failed reactions can play in devising successful reaction routes.

\subsection{Structural Verification \& Spectral Analysis}

In this section, we will first discuss the experimental results relating to the structural verification of each individual novel compound, then collectively compare the spectral data relating to electronic and chemical properties of the radical products with UV-VIS spectroscopy and cyclic voltammetry. Physical properties of the diradicals, such as magnetic susceptibility and conductivity are discussed in the subsequent section.

\subsection{1: 2,4-Diisopropyl-6-thiophene-1,2,4,5-tetrazane-3-one (3)}
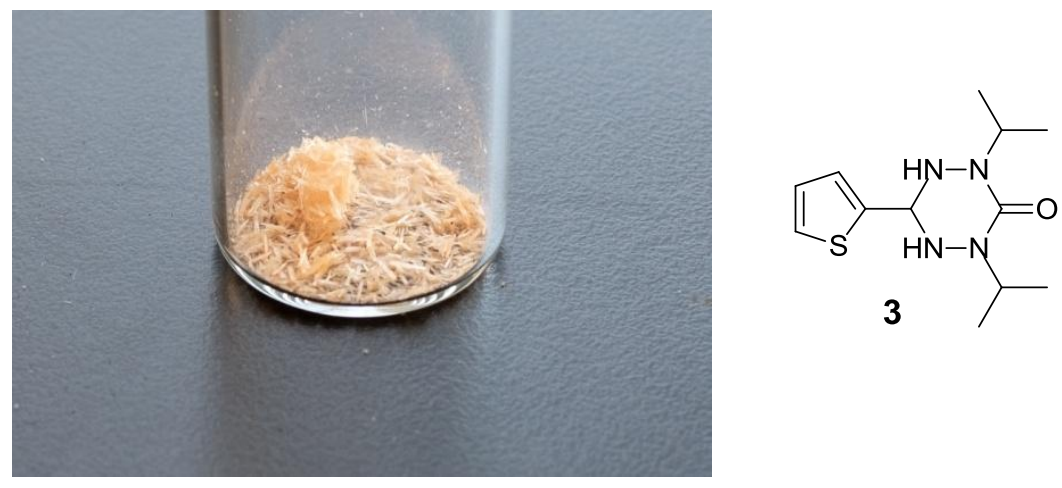

Figure 4.1 Recrystallized tetrazane (3).

The H-NMR spectrum for tetrazane 3 exhibits typical 2, 4-diisopropyl tetrazane peaks (H-NMR 1, Appendices), among which are the peaks due to isopropyl methyl hydrogens, which consist of two closely placed doublets in the alkane region, integrating to 6 
hydrogens each. The two methyl peaks are diastereotopic, and thus have slightly different chemical shifts. Other peaks associated with this type of tetrazane include, the signature septet peak due to the hydrogens on the secondary carbon of the isopropyl groups, which integrate to 2 hydrogens; a single doublet with two hydrogen integration due to secondary amine hydrogens; and finally, a triplet peak near the earlier septet peak, arising from the tertiary carbon hydrogen at the 3-position, which integrate to a single hydrogen. The peaks specific to tetrazane $\mathbf{3}$ are seen in the aromatic region, with two doublet peaks and a single doublet of doublet peak, each integrating to a single hydrogen. These peaks are exactly what we would expect for a mono-substituted thiophene, and taken together with the typical cyclic diisopropyl tetrazane peaks, strongly suggests the formation of tetrazane 3. C-NMR (C-NMR 1, Appendices) confirms the same structure with 9 nonequivalent carbon peaks located in regions characteristic of diisopropyl tetrazane and thiophene.

Results from IR spectroscopy further corroborate the formation of tetrazane $\mathbf{3}$ (IR Spectrum 1), with bands characteristic of 2, 4-diisopropyl tetrazanes at $3212 \mathrm{~cm}^{-1}$ for the amine bond $(\mathrm{N}-\mathrm{H})$, and a band at $1579 \mathrm{~cm}^{-1}$ for the carbonyl $(\mathrm{C}=\mathrm{O})$ bond. ${ }^{31}$ Mass spectrometry (GC-MS 1, Appendices) yielded a mass of $268 \mathrm{~m} / \mathrm{z}$, matching the calculated molecular weight for tetrazane 3 with the molecular formula $\mathrm{C}_{12} \mathrm{H}_{20} \mathrm{~N}_{4} \mathrm{OS}$. 


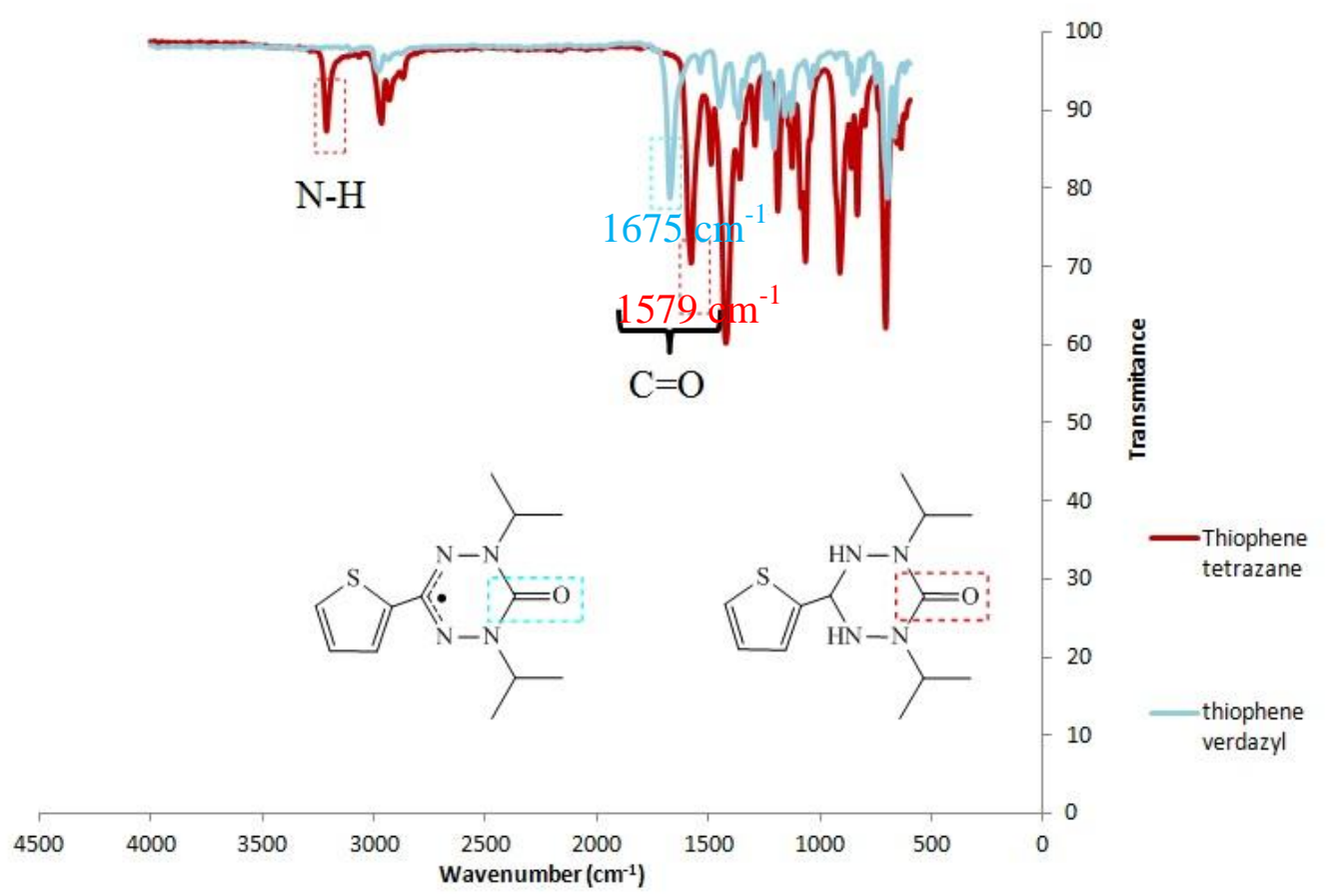

IR Spectrum 1: 2,4-Diisopropyl-6-thiophene-1,2,4,5-tetrazane-3-one (3) \& 1,5-Diisopropyl-3-thiophene-6-oxoverdazyl (4).

\subsection{2: 1,5-Diisopropyl-3-thiophene-6-oxoverdazyl (4)}
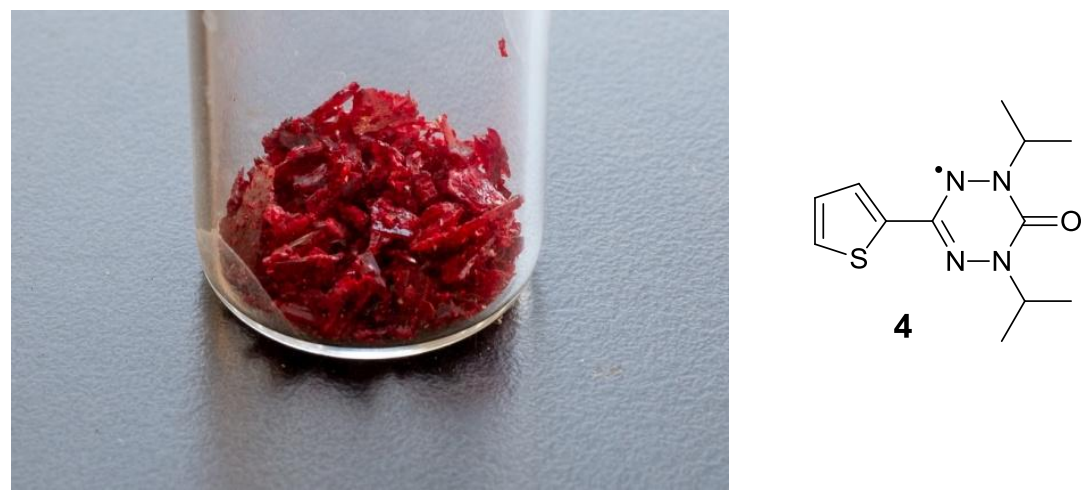

Figure 4.2 Recrystallized Verdazyl (4).

A qualitative indicator for the oxidation of tetrazane 3 to verdazyl 4 is the drastic color change from pale yellow to deep red, seen in Figure 4.2. More definitive evidence 
of verdazyl $\mathbf{4}$ formation is provided through spectral analysis. IR spectroscopy comparison of tetrazane $\mathbf{3}$ and its oxidized product (IR Spectrum $\mathbf{1}$ ) shows a clear leftward shift to higher wavenumbers for the $\mathrm{C}=\mathrm{O}$ carbonyl bond, indicating a comparatively higher bond order for the oxidized product compared to the starting tetrazane. The difference in $\mathrm{C}=\mathrm{O}$ band frequency between oxoverdazyls and their tetrazane precursors is well established, where tetraznes typically have $\mathrm{C}=\mathrm{O}$ bands in the mid $1500 \mathrm{~cm}^{-1}$ range, while the oxoverdazyls have $\mathrm{C}=\mathrm{O}$ bands in the mid $1600 \mathrm{~cm}^{-1}$ range. Furthermore, the amine $\mathrm{N}-\mathrm{H}$ band is no longer present in the oxidized product, as these amine hydrogens are removed in the oxidation process. Both IR changes are characteristic of oxoverdazyls.

The mere fact that the oxidized product gives an ESR reading is a confirmation that the structure contains unpaired electron(s), and is hence a radical, but ESR Spectrum 1 also provides structural information. The number of hyperfine coupling lines seen in an ESR spectrum depends on the atoms the unpaired electron interacts with. For a given element, the number of lines are given through the equation:

\section{Hyperfine line count $=2 N I+1$}

Here $N$ is the number of atoms for a given element, while $I$ is the nuclear spin of said element. In the case of a monoverdazyl, the unpaired electron primarily interacts with the four nitrogens, and since the nuclear spin of the most abundant nitrogen isotope is $I=1$, the hyperfine line count for monoverdazyls comes to:

$$
2(4)(1)+1=9 \text { lines }
$$


If the unpaired electron(s) interact strongly with more than one type of element, the ESR spectrum will be the result of overlapping lines from each element type.

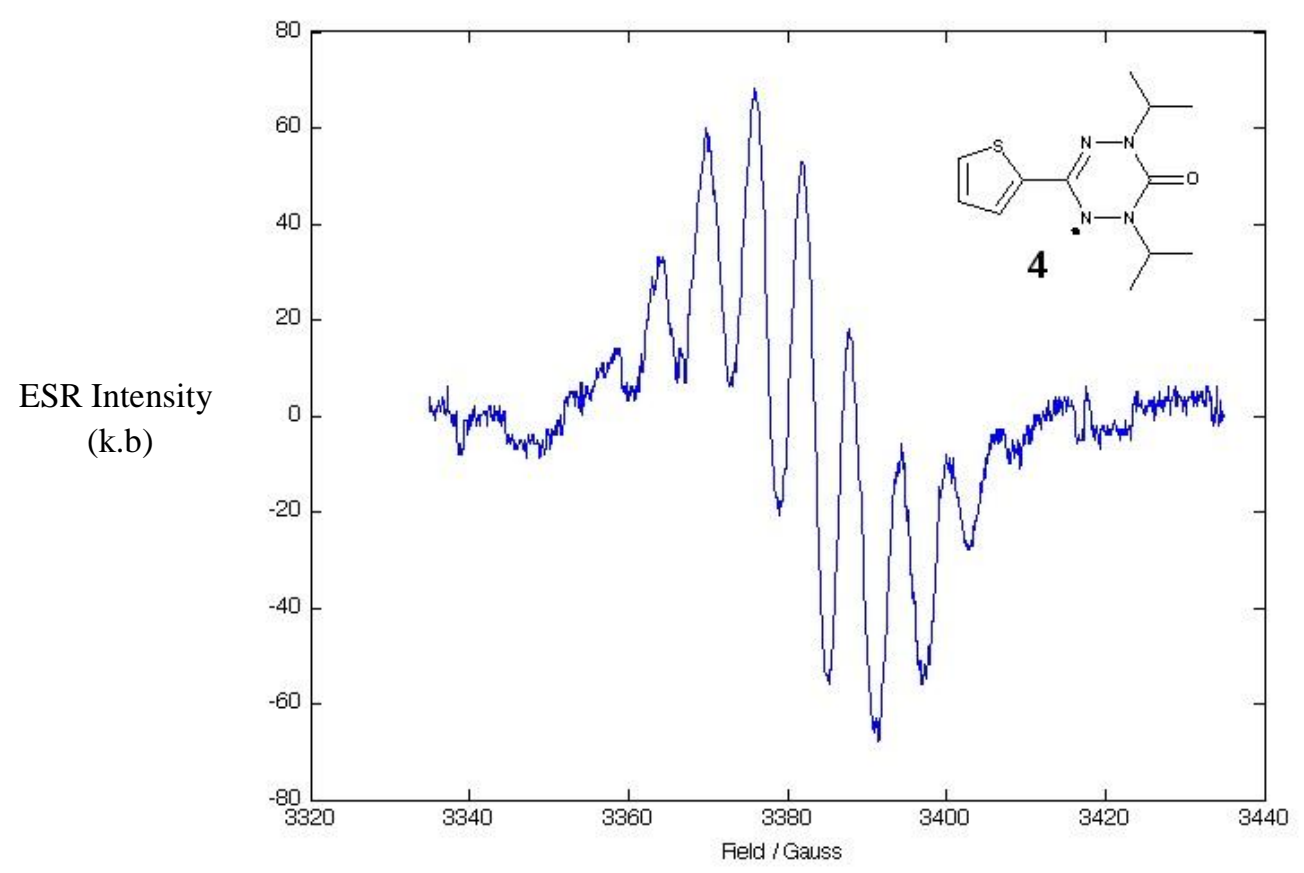

ESR Spectrum 1: 1,5-Diisopropyl-3-thiophene-6-oxoverdazyl (4).

The relative intensities of the hyperfine lines follow Pascal's triangle, and indeed the ESR spectrum for the oxidized product fits the above description closely with nine lines, providing more evidence for the formation of an oxoverdazyl.

Mass spectrometry (GC-MS 2, Appendices) of the oxidized product yielded $265 \mathrm{~m} / \mathrm{z}$. This mass value is 3 mass units lower than tetrazane $3(268 \mathrm{~m} / \mathrm{z})$, which would be appropriate for its corresponding verdazyl $\mathrm{C}_{12} \mathrm{H}_{17} \mathrm{~N}_{4} \mathrm{OS}$, as the process of oxidation to verdazyls removes three hydrogens; two amine hydrogens, and one hydrogen at the 3 position. The mass spectrum also provides a tidbit of structural information through fragmentation analysis. The first two fragments are $42 \mathrm{~m} / \mathrm{z}$, matching fragments due to 
isopropyl groups, a fragmentation pattern commonly observed for 1,5-diisopropyl oxoverdazyls. The structural verification of verdazyl 4 is rounded out with X-ray Crystallography (XRD). Figure 4.3 provides a 3D structural representation of verdazyl 4 based on the data provided by XRD (XRD Data 1, Appendices). Besides providing confirmation for the overall structure of verdazyl 4, the 3D image also shows the torsion angle between the verdazyl moiety and the thiophene ring $\left(23.66^{\circ}\right)$. Furthermore, Figure 4.3 shows a disorder of the thiophene ring, with the thiophene sulfur being present on both sides of the aromatic moiety. This suggests that both orientations of the thiophene with respect to the verdazyl ring are equally favored in the crystalline structure and chemically identical.

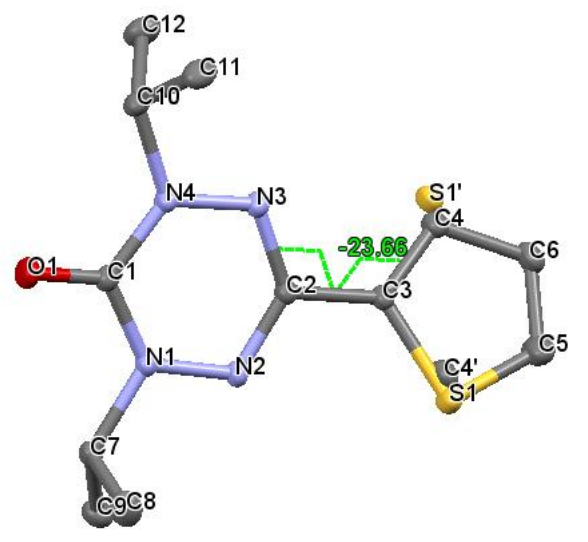

Figure 4.3 Verdazyl (4) Structure XRD. 
4.2.3: 1,5-Diisopropyl-3-thiophene-5-boronic acid pinacol ester-6-oxoverdazayl (5)
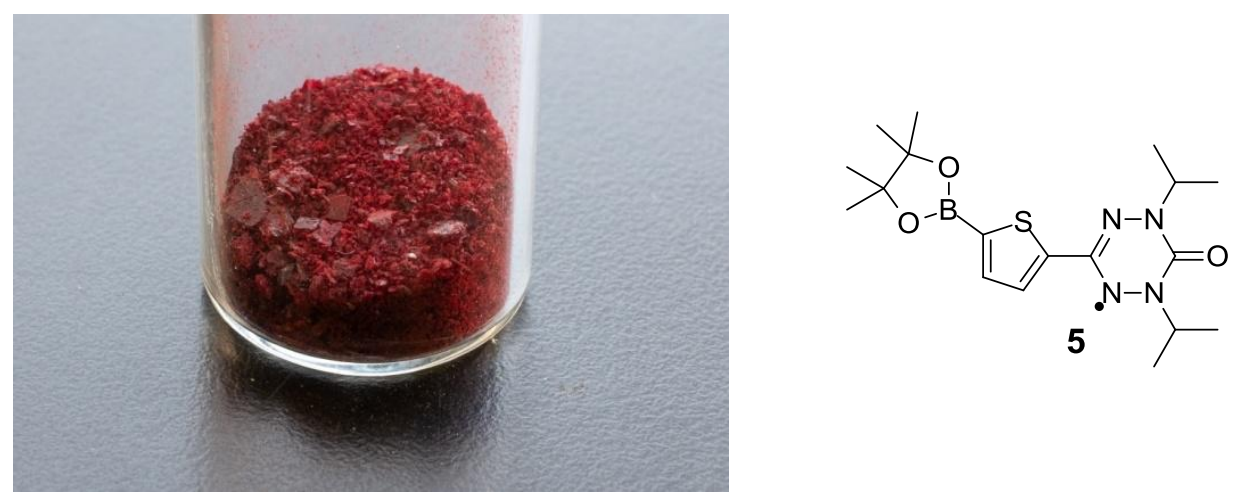

Figure 4.4 Recrystallized verdazyl (5).

Structurally verdazyl 5 very much resembles verdazyl 4, only differing in the inclusion of pinacol boronic ester moiety at the end of the thiophene. Unsurprisingly, when comparing the IR spectra of the two verdazyls we see nearly identical spectra with only minor differences in the fingerprint region (IR Spectrum 2). The important take away though is that both radicals have the characteristic verdazyl carbonyl band. 


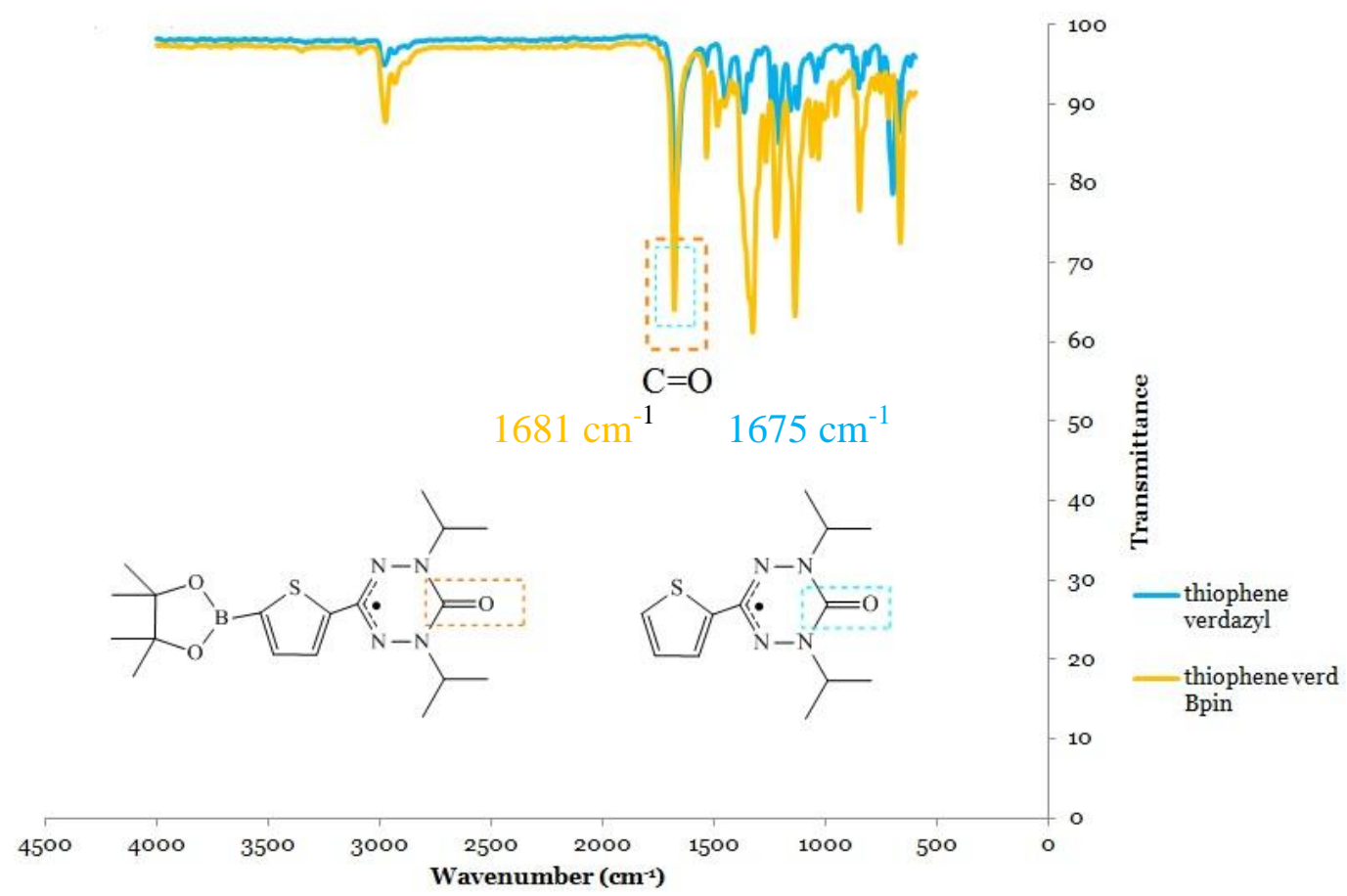

IR Spectrum 2: 1,5-Diisopropyl-3-thiophene-6-oxoverdazyl (4) \& 1,5Diisopropyl-3-thiophene-5-boronic acid pinacol ester-6-oxoverdazayl (5).

Similarly, ESR spectrum of the borylated product mirrors the non-borylated thiophene verdazyl (ESR Spectrum 2). This is to be expected, as the unpaired electron and its interactions are primarily limited to the verdazyl moiety and do not extend to the thiophene or the boronic ester, thus we see very similar ESR measurements. Fortunately, mass spectrometry can differentiate between the two verdazyls by providing the mass value $391 \mathrm{~m} / \mathrm{z}$ (GC-MS 3, Appendices). This value does match the calculated molecular weight for verdazyl 5 chemical formula $\mathrm{C}_{18} \mathrm{H}_{29} \mathrm{BN}_{4} \mathrm{O}_{3} \mathrm{~S}$. Similar to verdazyl $\mathbf{4}$, we see the characteristic successive isopropyl fragments in the mass spectrum for verdazyl $\mathbf{5}$, confirming the product as a diisopropyl oxoverdazyl. 


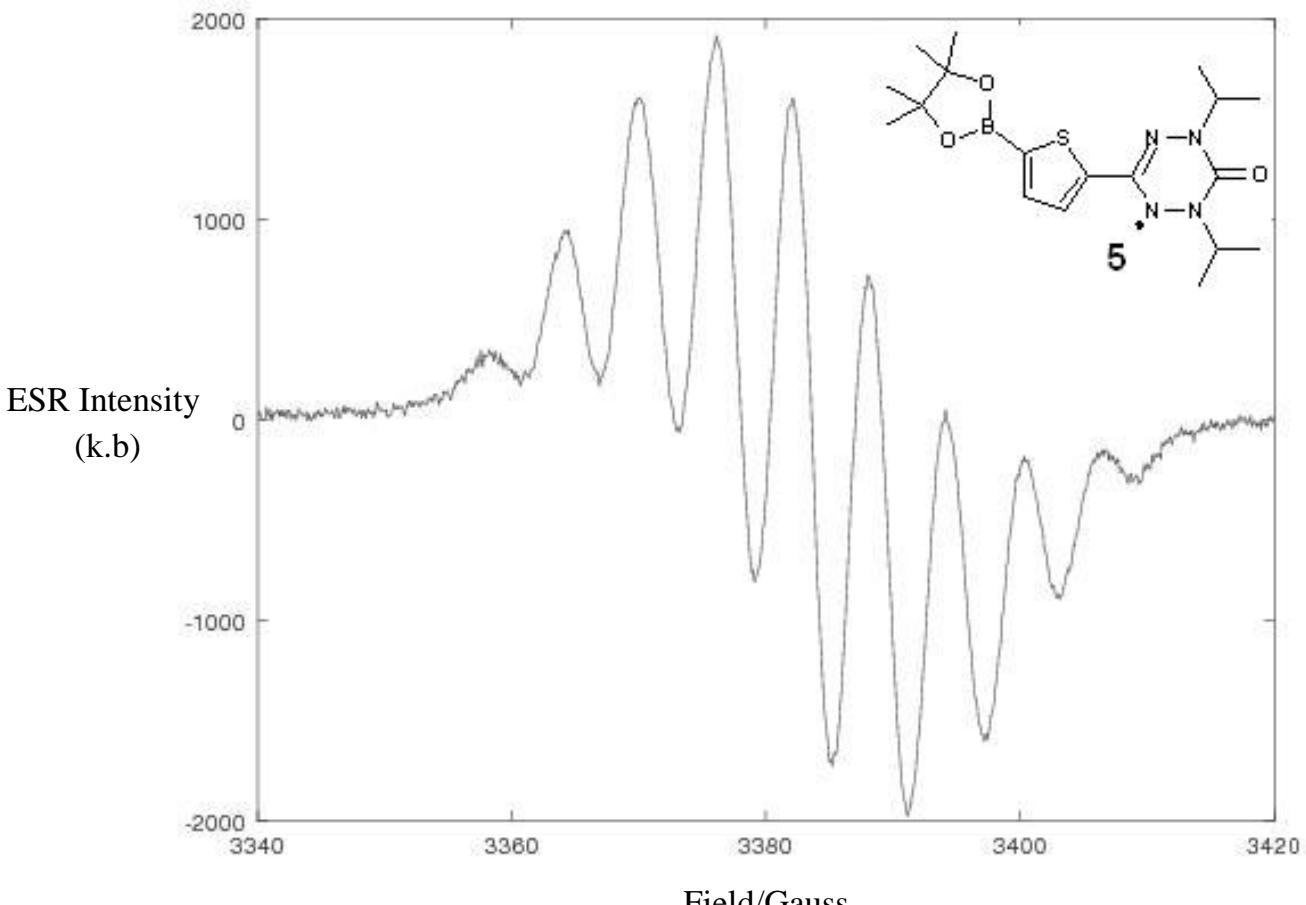

Field/Gauss

ESR Spectrum 2: 1,5-Diisopropyl-3-thiophene-5-boronic acid pinacol ester-6-oxoverdazayl (5).

The definitive proof for verdazyl 5 is provided by XRD (XRD Data 2, Appendices). As seen in the 3D structure of Figure 4, not only is the addition of the pinacol boronic ester confirmed, but we also see the angle between the thiophene, verdazyl ring and the boronic ester is fixed, unlike verdazyl 4. Interestingly, the torsion angle between the thiophene ring and the verdazyl ring has also been reduced in comparison to verdazyl 4, making the two rings more co-planar. The thiophene and the pinacol boronic ester are also largely planar with respect to one another. 


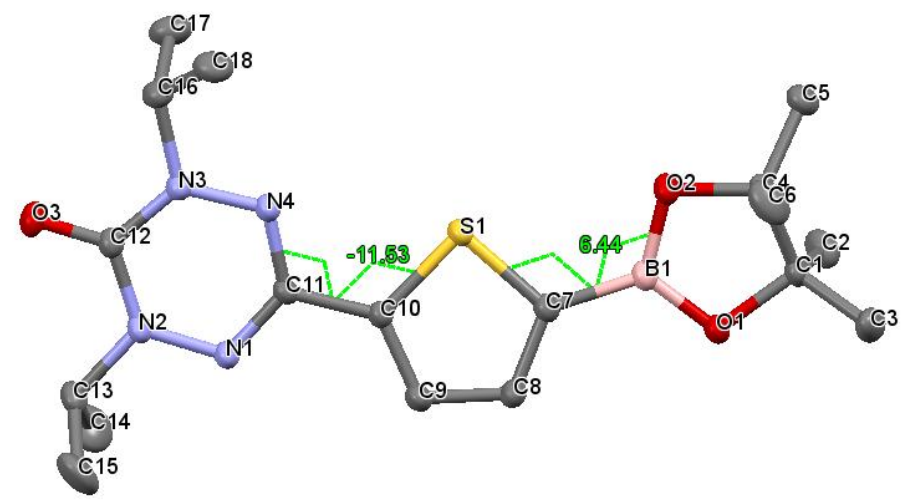

Figure 4.5 Verdazyl (5) structure XRD.

While the iridium catalyzed borylation of the thiophene-verdazyl did succeed in forming the desired product, the yields obtained through this reaction route proved modest. Two factors have been identifies as the likely culprit for the reduced yields. The first hurdle was the partial conversion of the thiophene verdazyl to its borylated form (50$60 \%)$, in all but our final attempt (80-90\% conversion). The product conversion was found to be tangibly improved with more rigorous degassing and higher catalyst loading. Unfortunately, the initial starting material had been consumed by this juncture. The second factor reducing yield was the difficulty of separating residual verdazyl $\mathbf{4}$ from verdazyl 5 product. Due to their very similar structure and polarity, flash chromatography of the crude product results in the overlapping of the two verdazyl components. Ultimately a portion of product 5 eluted with residual verdazyl $\mathbf{4}$, lowering the purified yield further. An improved conversion percentage might allow for traditional recrystallization techniques in future attempts, which may circumvent the troubles of overlapping column elution, and increase final yield. All things considered though, while the successful iridium borylation of an aryl-verdazyl was an important milestone in our 
research, our ultimate goal is the synthesis and study of conjugated bisverdazyls, hence ensuring a decent yield of verdazyl 5 was a major objective. Because of this we devised an alternative synthesis route where borylation occurs before tetrazane and verdazyl formation.

\subsection{4: 2,4-Diisopropyl-6-thiophene-5-boronic acid pinacol ester-1,2,4,5-tetrazane-3- one (4')}
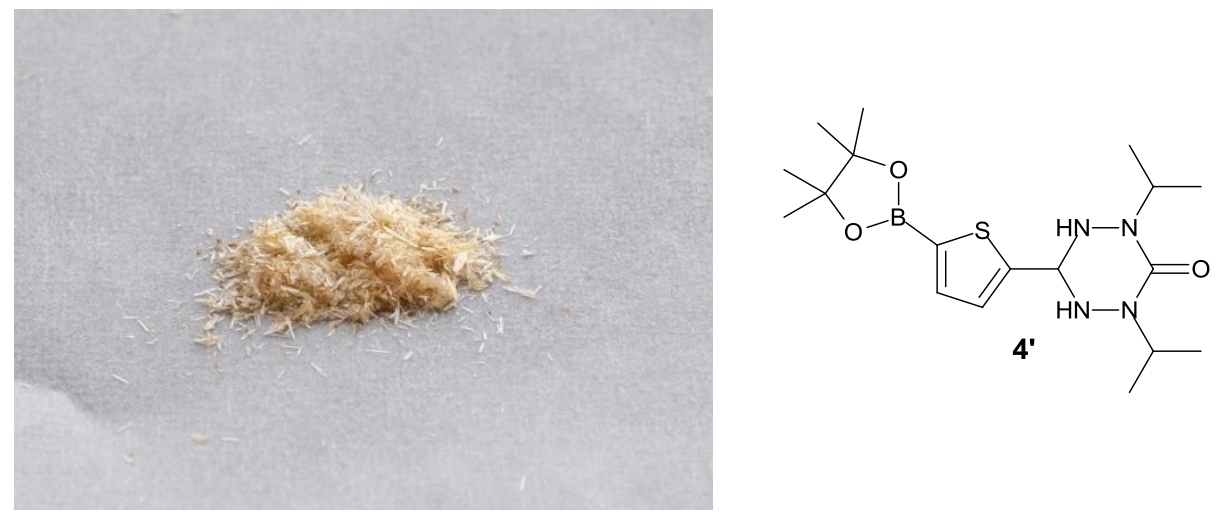

Figure 4.6 Recrystallized tetrazane (4').

We will not discuss the characterization 5-formyl-2-thienylboronic acid pinacol ester (3') in depth, as this product is commercially available (see Scheme 3.4 for structure). Instead we will merely verify its successful synthesis via H-NMR and Mass Spectroscopy. H-NMR 2 (Appendices) easily confirms the formation of carboxaldehydeboronic ester $3^{\prime}$ with a singlet aldehyde peak in $10 \mathrm{ppm}$ region integrating to one hydrogen, two doublet peaks in the aromatic region due to the thiophene hydrogens (1 hydrogen each), and a large singlet peak in alkane region (integrating to 12 hydrogens), for the pinacol boronic ester methyl hydrogens. GC-MS 4 (Appendices) also confirms the structure with $238 \mathrm{~m} / \mathrm{z}$ mass, matching the known molecular weight of 5-formyl-2thienylboronic acid pinacol ester (3'). Furthermore, a cursory fragmentation analysis 
lends more support for the structure, with the first fragment of $15 \mathrm{~m} / \mathrm{z}$ suggesting methyl fragments from the pinacol boronic ester end of the structure. Boronate $\mathbf{3}^{\prime}$ is not a novel compound, but its formation is significant in two ways. First, it is the result of converting less soluble boronic acid to its corresponding pinacol boronic ester, which improves its solubility in non-polar solvents, and is known for greater stability in silica based column chromatography. Second, boronate 3 ' provides the borylated aldehyde containing thiophene needed for the formation of our desired borylated tetrazane.

Having established the formation of carboxaldehyde $3^{\prime}$, we can now discuss the characterization of its resulting tetrazane. The first clue to the formation of tetrazane $\mathbf{4}^{\prime}$ is provided by H-NMR 3 spectrum (Appendices) with the disappearance of the aldehyde peak at $10 \mathrm{ppm}$ range. Most peaks for this product mirror those of tetrazane 4, except for two peaks that allow us to differentiate the two tetrazanes. First, tetrazane $4^{\prime}$ only has two doublet aromatic peaks, while tetrazane $\mathbf{4}$ has three aromatic peaks with one being a doublet of a doublet. This is of course because the thiophene in tetrazane $4^{\prime}$ is substituted in both $2 \& 5$ position, while the thiophene in tetrazane 4 is only substituted at the 2 position. Second, with tetrazne 4' we see an additional singlet peak in the alkane region (integrated to 12 hydrogens) due to the methyl hydrogens of the pinacol boronic ester, which is absent in tetrazane 4. A similar trend is observed with Carbon NMR, where CNMR 2 (Appendices) shows a very similar spectrum to that of tetrazane 4 except with two additional peaks (11 total, one appears hidden by solvent peak) due to the boronic ester moiety. 
IR Spectroscopy of tetrazane 4' (IR Spectrum 3) is nearly identical to tetrazane 4, save for minor variances in the fingerprint region, which is in line with our expectation for this type of tetrazane, with characteristic bands for Carbonyl $\mathrm{C}=\mathrm{O}$ in the $1500 \mathrm{~s} \mathrm{~cm}^{-1}$ range and amine $\mathrm{N}-\mathrm{H}$ bands in $3200 \mathrm{~s} \mathrm{~cm}^{-1}$ range. Concluding our structural verification, GC-MS 5 (Appendices) found the mass of tetrazane 4' to be $394 \mathrm{~m} / \mathrm{z}$. This mass would agree with the expected value for the corresponding tetrazane to verdazyl $\mathbf{5}$, where the mass is 3 mass units heavier than verdazyl 5 mass of $391 \mathrm{~m} / \mathrm{z}$.

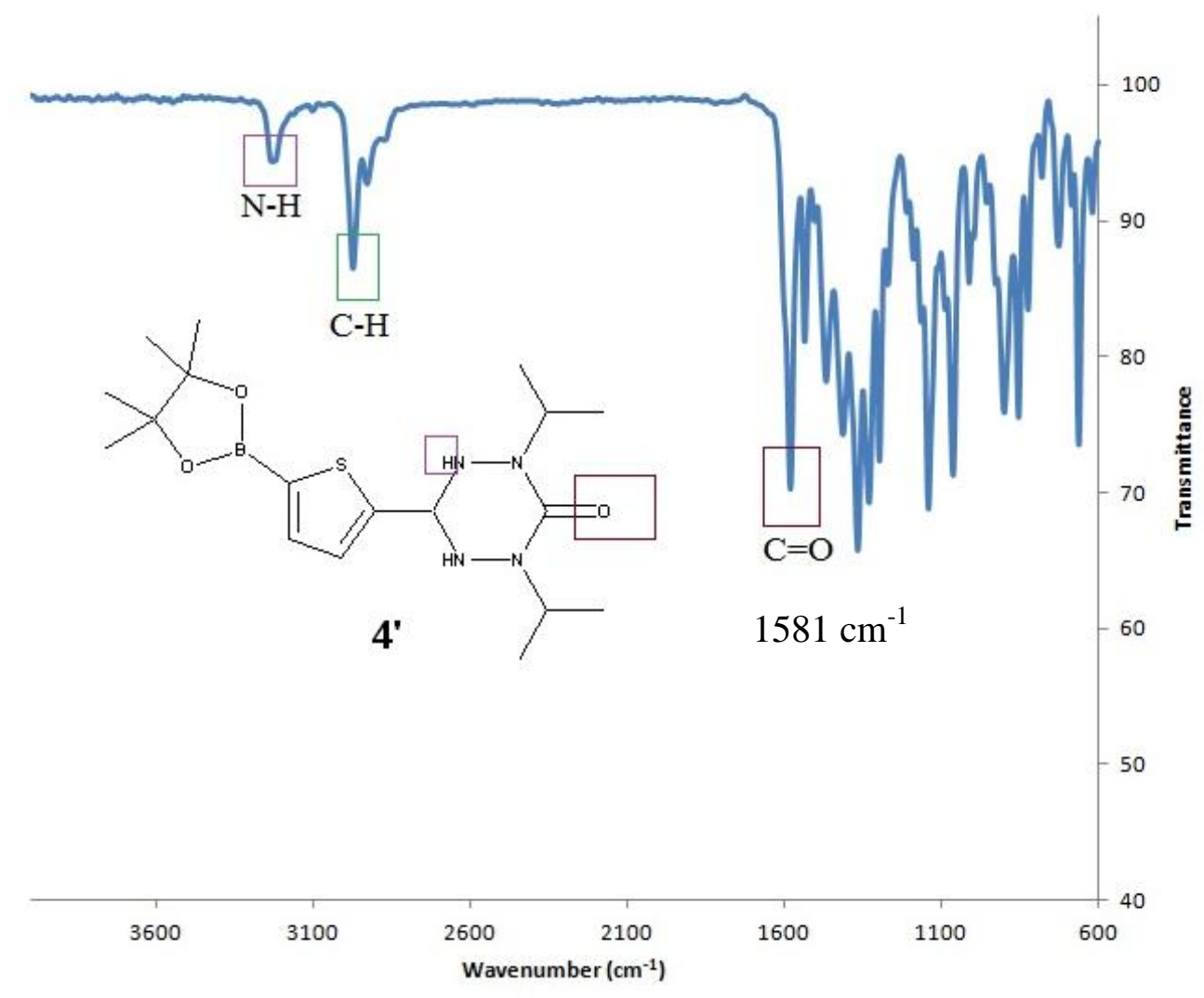

IR Spectrum 3: 2,4-Diisopropyl-6-thiophene-5-boronic acid pinacol ester-1,2,4,5-tetrazane-3-one (4').

A follow up oxidation step converts tetrazane 4 ' to verdazyl 5 . We have not included a repeat of the spectra for verdazyl $\mathbf{5}$ in the Results and Appendices sections, however, 
it's worth emphasizing the greater yield obtained through this alternative synthesis route compared to the initial synthesis route involving Iridium catalyzed borylation of the thiophene-verdazyl (49\% vs 14\% respectively). We believe the greater yield to be at least in part due to the more facile purification of the verdazyl from its tetrazane precursor compared to two similar verdazyls. Thus, it can be concluded that for the synthesis of borylated-aryl-verdazyls, its preparation from a tetrazane precursor is likely to yield more product, although if such an option were unavailable, iridium borylation of the arylverdazyl is still a viable option.

\subsection{5: Bis (1, 5-diisopropyl-6-oxoverdazyl)-3,3'- $\alpha$-quaterthiophene (6)}

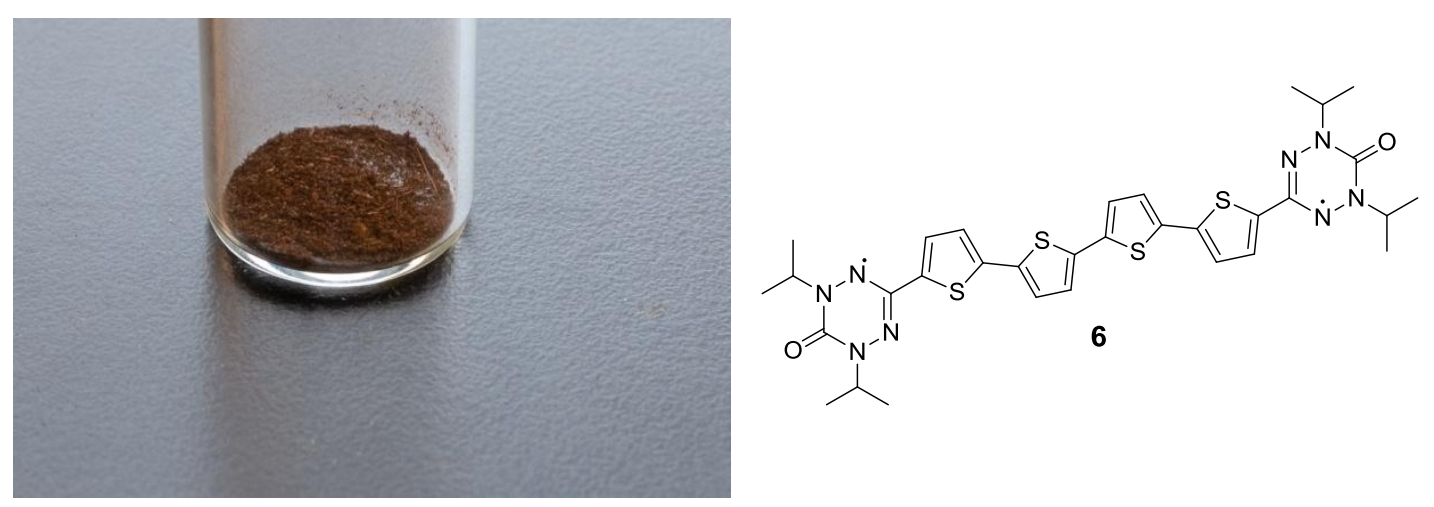

Figure 4.7 Recrystallized Bisverdazyl (6).

With the synthesis of a fair amount of borylated verdazyl $\mathbf{5}$, we could proceed with double Suzuki reaction to form our target diradical, bisverdazyl 6. IR Spectroscopy confirms the product as a verdazyl (IR Spectrum 4) with the characteristic carbonyl $\mathrm{C}=\mathrm{O}$ band, but cannot verify whether the structure is a monoradical or a diradical. 


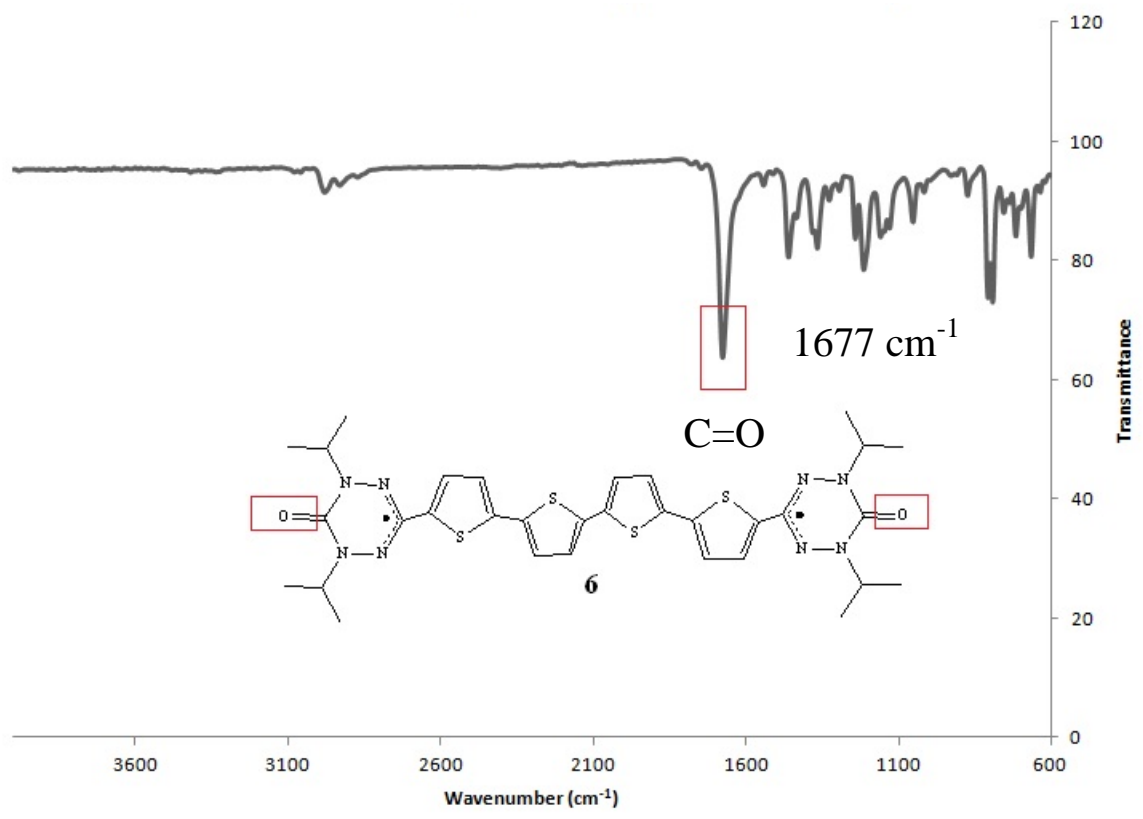

IR Spectrum 4: Bis (1, 5-diisopropyl-6-oxoverdazyl)-3,3'- $\alpha$ quaterthiophene $(\mathbf{6})$.

Instead the evidence for diradical formation is provided by ESR spectroscopy (ESR Spectrum 3), where the spacing between the hyperfine coupling lines are nearly halved for bisverdazyl 6, compared to previous spectra for monoradicals. 


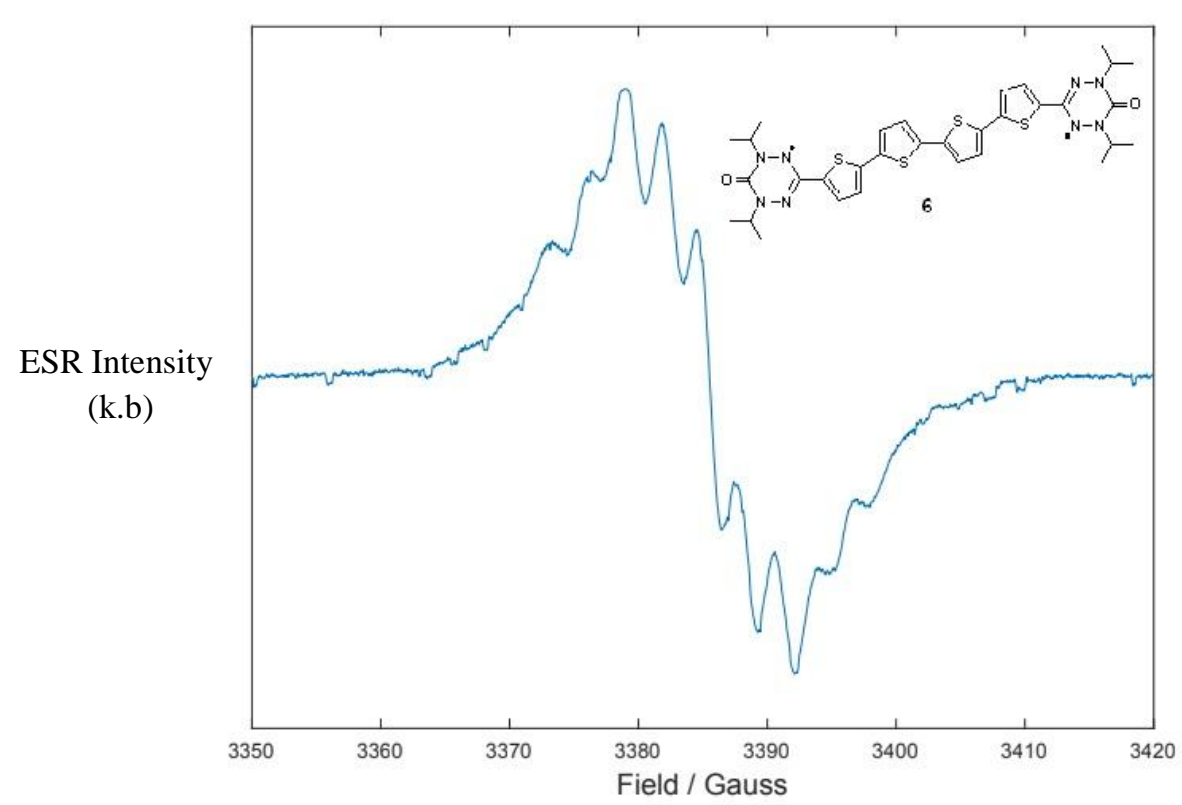

ESR Spectrum 3: Bis (1, 5-diisopropyl-6-oxoverdazyl)-3,3'- $\alpha$ quaterthiophene (6).

The difference seen between the ESR spectra of bisverdazyls and monoverdazyls, is due to each unpaired electron interacting with 8 nitrogens in bisverdazyls, instead of only 4 with monoverdazyls. The strength of the unpaired electron interactions with the nitrogens of the opposite verdazyl depends on the proximity of the two verdazyl moieties and delocalization of the separating spacer. A bisverdazyl can give an ESR spectrum very similar to monoverdazyls if the two verdazyls are separated by lengthily spacer molecules or an insulating moiety. Conversely very closely placed verdazyls would result in a broadening of the hyperfine lines. In the case of bisverdazyl $\mathbf{6}$, the two radicals do indeed interact, though relatively modestly.

Mass spectrometry of bisverdazyl 6 (LC-MS 1, Appendices) yielded a mass value of $695 \mathrm{~m} / \mathrm{z}$, which is 3 mass units greater than what would be expected for bisverdazyl 6. It 
is not uncommon for verdazyls to undergo reduction to leucoverdazyls under certain conditions, and here we suspect that the solvent matrix and LC-MS instrumentation may have led to reduction of bisverdazyl 6 to its bis (leucoverdazyl) equivalent, plus an additional reduction with an extra hydrogen. Despite the unintended reduction, the LCMS results further support the formation of bisverdazyl $\mathbf{6}$.

The full Structure of bisverdazyl 6 was verified with X-ray crystallography. In Figure 4.8 we can see the three-dimensional structure provided by XRD, where two diisopropyl oxo-verdazyls are separated by a thiophene tetramer spacer. Just as importantly the figure also shows the torsion angle between the thiophene units and the verdazyls. The torsion angles between the thiophenes are quite small, such that the thiophene chain can be regarded as roughly planar, and thus conjugation along the thiophenes is intact. But, unlike the conventional way thiophene chains are represented with trans orientation (seen with our structural schemes earlier), here each two thiophenes on either half of the structure have cis conformation, while only the central thiophenes have trans conformation with respect to one another. This gives bisverdazyl $\mathbf{6}$ symmetry with respect to a plane passing through the middle of the structure. Additionally, we can note that the verdazyl moieties are not entirely in plane with the thiophene chain. 


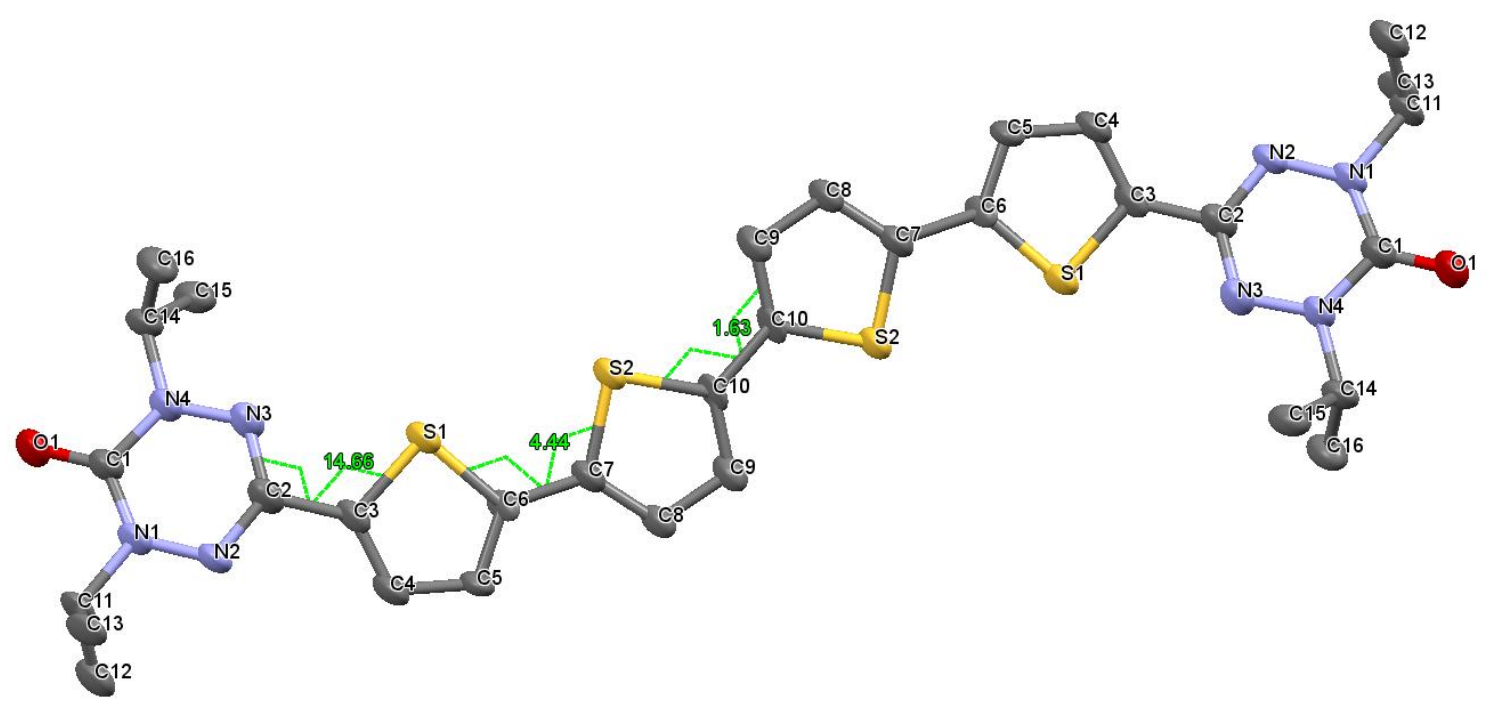

Figure 4.8 Bisverdazyl (6) structure XRD.

XRD also provides a look at molecular packing (not pictured). Monoverdazyls 4 \& 5 exhibited typical Herringbone packing (slanted head to tail), with intermolecular contact points primarily consisting of alkyl group hydrogens and carbonyl oxygens, this type of intermolecular packing is often detrimental to intermolecular conduction. ${ }^{43}$ Bisverdazyl 6 on the other hand displayed a packing pattern which can be describe as an intermediate between planar and herringbone, this packing pattern indicates some degree of $\pi$-stacking due to the longer conjugated thiophene chain. Intermolecular packing is especially important for OSCs, which face some conductivity hurdles due to incoherent hopping charge transport mechanism. We will discuss the significance of packing further in our future works section. 


\subsubsection{Bis(1,5-diisopropyl-6-oxoverdazyl)-3-3'-bithiophene-4,4'-biphenyl (7)}
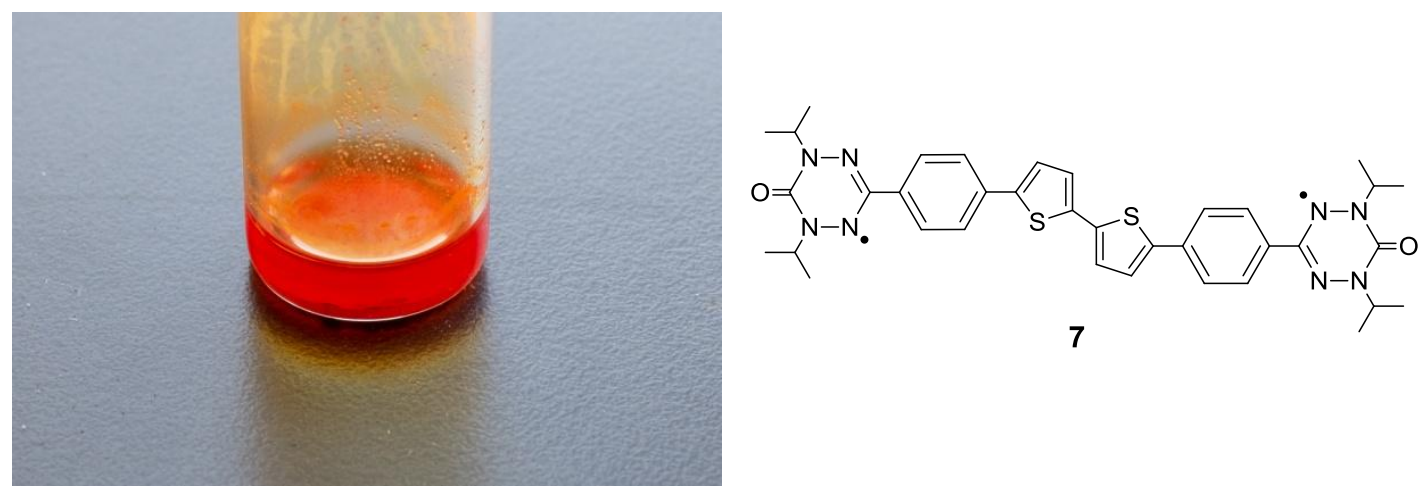

Figure 4.9 Bisverdazyl (7) in xylene.

The Brook research group previously synthesized borylated phenyl-verdazyl 5' while working on the Le et al. paper. ${ }^{30}$ Given our success with the double Suzuki reaction when synthesizing bisverdazyl $\mathbf{6}$, we sought to synthesize a second bisverdazyl for comparison by reacting borylated verdazyl $\mathbf{5}^{\prime}$ and 5,5'-dibromo-2,2'-bithiophene in a double Suzuki coupling reaction.

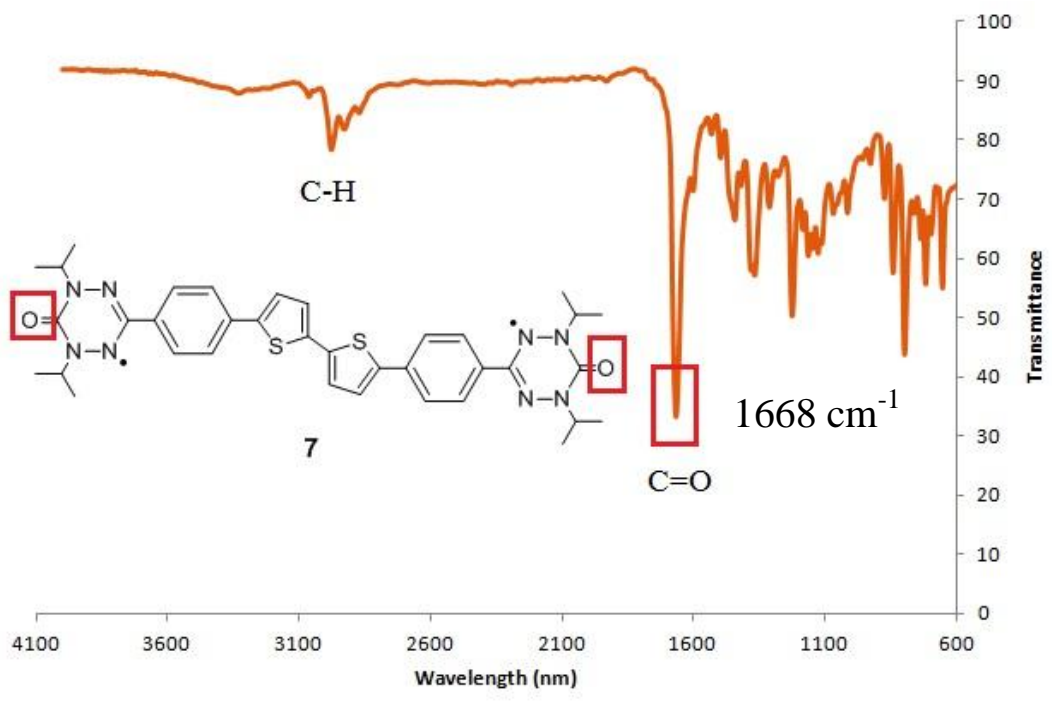

IR Spectrum 5: Bis(1,5-diisopropyl-6-oxoverdazyl)-3-3'-bithiophene4,4'-biphenyl (7). 
Just as with bisverdazyl 6, IR spectroscopy of bisverdazyl 7 (IR Spectrum 5) confirms the product as an oxoverdazyl with the characteristic carbonyl $\mathrm{C}=\mathrm{O}$ band, while ESR Spectrum 4 identifies $\mathbf{7}$ as a bisverdazyl with a very similar spectrum to bisverdazyl $\mathbf{6}$. The small secondary splitting on top of the hyperfine lines of ESR Spectrum 4 are due the minor interactions of the unpaired electrons with the verdazyl isopropyl hydrogens, and is present in all diisopropyl verdazyl ESR spectra, but is more apparent here due to better sample degassing.

We were unable to obtain XRD measurements for bisverdazyl 7, as the small amount of product prevented the effective growth of XRD grade crystals through diffusion recrystallization. However, studies with mixed thiophene/phenyl oligomer-diradical systems have shown that the inclusion of phenyl rings lead to decrease in oligomer chain planarity and conjugation. ${ }^{44}$ Phenyl rings in conjugated oligomers often adopt tilted conformations due to the sterics of the hydrogens on either side of the aromatic ring. This is less of an issue for thiophens, as they are considerably more compact with only two hydrogens on one side of the ring, and thus usually adopt planar conformation with one another in poly/oligomers. 


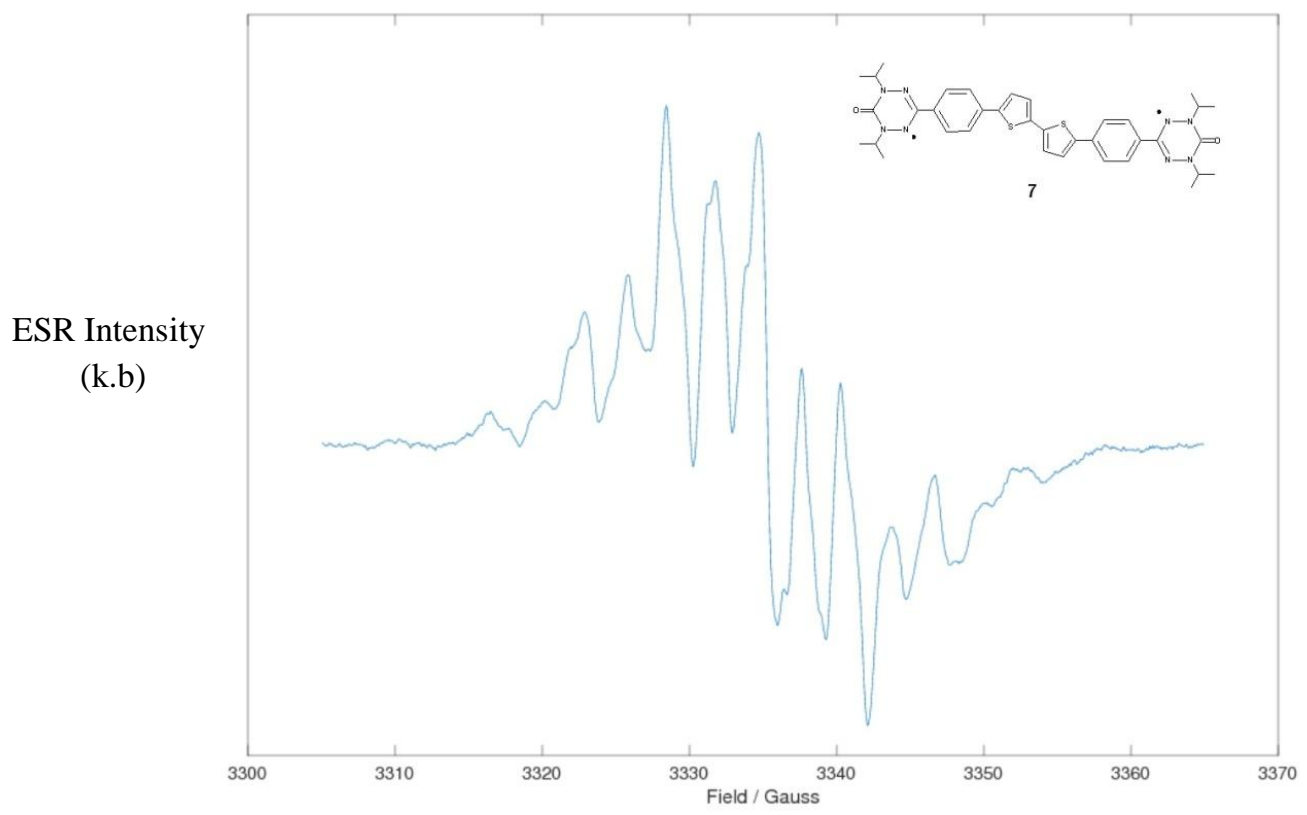

ESR Spectrum 4: Bis (1, 5-diisopropyl-6-oxoverdazyl)-3,3'-biphenyl4,4'-bithiophene (7).

\subsubsection{UV-VIS Spectra}

For organic molecules, absorption in the ultraviolet-visible range results from electronic transitions of valence electrons from ground states to excited states. While electronic transitions for valence electrons encompass $\sigma \rightarrow \sigma^{*}$ transitions as well, the energy requirements for these transitions place their absorbances outside of typical UVVIS spectra ranges (200-700 nm). The most common transitions in the UV-VIS range are instead those due to conjugated systems with $\pi \rightarrow \pi^{*}$ transitions, or unpaired/lone pair electron transitions such as $n \rightarrow \pi^{*}$ or $n \rightarrow \sigma^{*}$ as seen in Figure 4.10. Because our verdazyls have both unpaired electrons and conjugated components, all three of the transitions in Figure 4.10 may be present in their UV-VIS spectra, although $n \rightarrow \sigma^{*}$ transitions are less prevalent. 


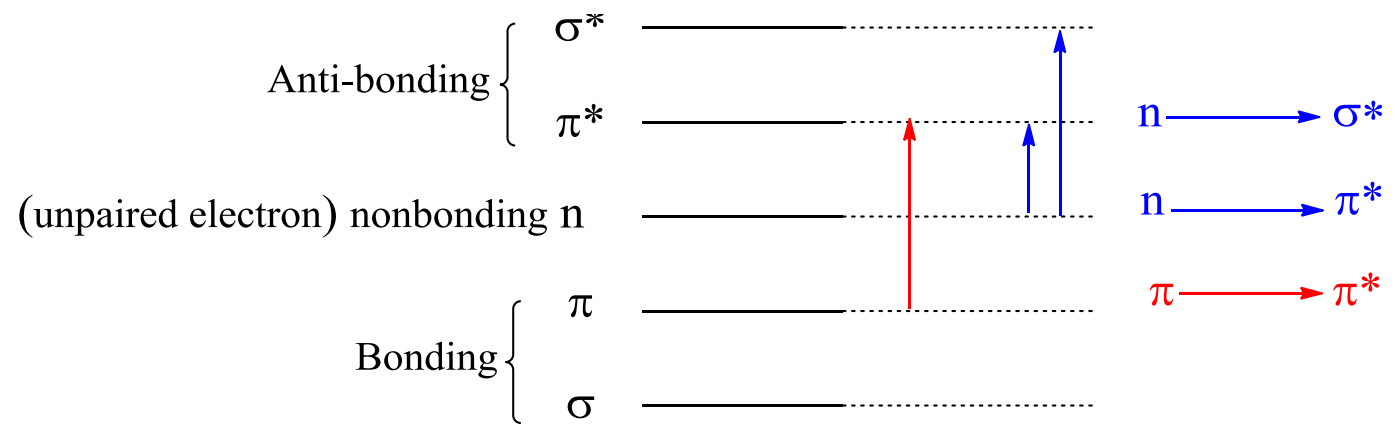

Figure 4.10 Electronic transitions due to UV-VIS absorption.

UV-VIS Spectrum 1-4 (Appendices) show the spectra for each of the radical species, with absorption maxima and corresponding (likely) transition type labeled. The absorbance maxima for all radical species are listed in Table 4.1, along with the extinction coefficients (except for bisverdazyl 7).

Table 4.1 UV-VIS peak maxima for radical species.

\begin{tabular}{lll}
\hline Radical Species & \multicolumn{1}{c}{$\begin{array}{c}\lambda \max _{1}[\mathrm{~nm}] \\
(\varepsilon[\mathrm{L} / \mathrm{mol} . \mathrm{cm}])\end{array}$} & $\begin{array}{c}\lambda \max _{2}[\mathrm{~nm}] \\
(\varepsilon[\mathrm{L} / \mathrm{mol} . \mathrm{cm}])\end{array}$ \\
\hline Verdazyl 4 & $426\left(1.2 \times 10^{3}\right)$ & $514\left(4.0 \times 10^{2}\right)$ \\
Verdazyl 5 & $430\left(1.3 \times 10^{3}\right)$ & $520\left(4.6 \times 10^{2}\right)$ \\
Bisverdazyl 6 & $428\left(5.5 \times 10^{4}\right)$ & $592\left(7.7 \times 10^{2}\right)$ \\
Bisverdazyl 7 & 398 & 510 \\
\hline
\end{tabular}

*Note: The Molar extinction Coefficent for Bisverdazyl 7 was not obtained due to small product yield.

Relation between the magnitude of photon energy and wavelength is inverse, what this means is that smaller energy gap transitions undergo absorptions in longer wavelengths, while larger gap transitions require shorter wavelength absorptions. Absorbances at higher wavelengths are due to transitions with either particularly high energy HOMO orbitals or low LUMO orbitals. Unpaired SOMO electrons are one such 
high-energy non-bonding HOMO orbitals, with small energy gaps separating them from their respective LUMO orbitals, and so transitions for such electrons are often seen in high wavelengths, especially for $n \rightarrow \pi^{*}$ transitions. Unfortunately, due to the low extinction coefficient of such transitions, their absorption peaks are difficult to identify as they are swamped by other transitions with much higher extinction coefficients such as $\pi \rightarrow \pi^{*}$ transitions. Nevertheless, such transitions certainly occur for the verdazylconjugated systems.

The absorptions seen in Table 4.1 are all likely due to various $\pi \rightarrow \pi^{*}$ transitions brought about due to the aromatic rings and conjugated oligomers. A look at the maximum absorbance values for verdazyl $4 \& 5$ makes it clear that the two radical systems have very similar transitions, with verdazyl $\mathbf{5}$ only slightly red shifted in comparison to verdazyl 4 . This is very much expected, as the addition of a boronic ester is not likely to alter the electronic configuration of the thiophene or verdazyl to a noticeable extent.

The bisverdazyls show a much stronger absorption with high extinction coefficients compared to the mono-radicals, it is also noteworthy that the second absorption for bisverdazyl 6 at $592 \mathrm{~nm}$ is significantly red shifted compared to the mono-radicals, which is likely due to the higher conjugation length shrinking band gap for this transition. Comparing the UV-VIS spectra of bisverdazyl 6 \& 7, we see that the latter diradical is blue shifted in comparison to the former in the visible range. This is because quarterthiophene is more conjugated than biphenyl-bithiophene due to its planar structure, while the latter oligomer has a more twisted structure due to the phenyl rings. ${ }^{32}$ 
Interestingly though, bisverdazyl 7 exhibits a red shifted peak in the UV range (not pictured) compared to bisverdazyl $\mathbf{6}$, suggesting that a second more energetic transition in the UV range of bisverdazyl 7 may have a smaller gap than that of bisverdazyl $\mathbf{6}$.

As a final point to conclude our UV-VIS discussion, the absorptions in the visible range not only determines some of the electronic properties of radical species, they also determine the observed color of substances. The color we witness is the reflected complementary colors to the visible light absorbed by substances (see Figure 4.11).

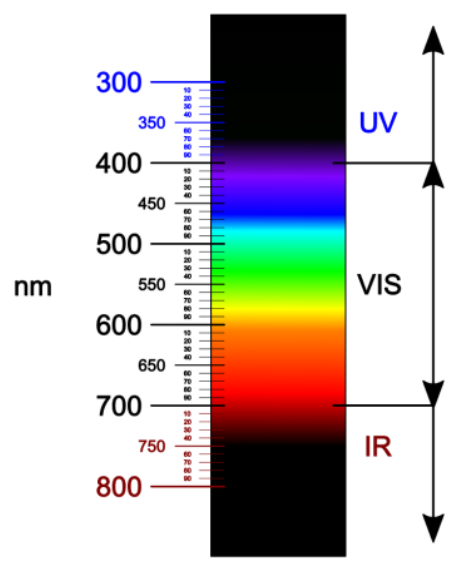

Figure 4.11 UV-VIS-IR light spectrum by Fulvio314 - Own work, CC BYSA 4.0,https://commons. wikimedia.org/w/index.php?curid=50181281.

For instance, verdazyl 4 absorbs visible light to various degrees in the range of 380$600 \mathrm{~nm}$. That leaves visible light in the $600-700 \mathrm{~nm}$ region to reflect off the solid, and thus we observe the radical as red colored. We see a very similar color for verdazyl $\mathbf{5}$ as well, which also agrees with its absorbance in the visible range. Bisverdazyl $\mathbf{6}$ is a bit more complicated, as it has a wide range of absorptions across almost the entirety of the visible range, so much so that the solid has a generally dark gray coloration as most of the visible light is absorbed. But, absorption beyond the high 500s $\mathrm{nm}$ are relatively small for 
bisverdazyl 6, such that a mild yellow colored luster is observed with this diradical. Bisverdazyl 7 on the other hand has a shorter range of absorptions in the visible range up to $\sim 580 \mathrm{~nm}$ and as such it is observed as a bright orange colored solid.

\subsubsection{Cyclic Voltammetry}

Cyclic voltammetry (CV) spectra display the current profile of a substance while it undergoes changes in its oxidation state as a result of potential sweeps by the working electrode. CV can be used to study electrochemical properties of a substance in a wide range of ways, from kinetics to concentration determinations. Our analysis of the spectra will however focus on redox half potentials $\mathrm{E}_{1 / 2}$, redox process reversibility, and product stability.

Table 4.2 displays the $\mathrm{E}_{1 / 2}$, Epa, and Epc values, which are respectively the half wave potential, oxidation potential, and reduction potential for each $\mathrm{CV}$ wave of the novel radicals. Cyclic voltammetry can be used to estimate band gap and HOMO/ LUMO levels for organic materials. The difference between two successive half wave potentials for instance, can give a rough estimate of the band gap for an electronic transition within a given potential range. A cursory comparison of the only two $\mathrm{CV}$ runs with reversible redox waves in Table 4.2, verdazyl $\mathbf{4}$ and bisverdazyl 6, shows that the band gap of bisverdazyl 6 is notably smaller at $1.64 \mathrm{v}$, compared to $1.73 \mathrm{v}$ for verdazyl 4 . This result agrees with the findings seen in UV-VIS spectrometry, where bisverdazyl $\mathbf{6}$ absorbs in longer wavelengths, indicative of a smaller band gap transitions. Of course, the above results are to be expected considering bisverdazyl $\mathbf{6}$ is more extensively conjugated than verdazyl 4. 
Table 4.2 Cyclic voltammetry potentials for radical species.

\begin{tabular}{lccccccc}
\hline Radical Species & $\mathrm{Epa}_{1^{*}}(\mathrm{Ox})$ & $\mathrm{Epc}_{1}(\mathrm{Red})$ & $\mathrm{Epa}_{1}(\mathrm{Ox})$ & $\mathbf{E}_{1 / 2(1)}(\mathbf{V})$ & $\mathrm{Epc}_{2}(\operatorname{Red})$ & $\mathrm{Epa}_{2}(\mathrm{Ox})$ & $\mathbf{E}_{1 / 2(2)}(\mathbf{V})$ \\
\hline Verdazyl 4 & $\mathrm{n} / \mathrm{a}$ & 0.26 & 0.52 & 0.39 & -1.45 & -1.22 & -1.34 \\
Verdazyl 5 & $\mathrm{n} / \mathrm{a}$ & 0.13 & 0.61 & 0.37 & -1.58 & $\mathrm{n} / \mathrm{a}$ & $\mathrm{n} / \mathrm{a}$ \\
Bisverdazyl 6 & $\mathrm{n} / \mathrm{a}$ & 0.22 & 0.50 & 0.36 & -1.37 & -1.2 & -1.28 \\
Bisverdazyl 7 & 0.99 & 0.43 & 0.51 & 0.47 & -0.54 & $\mathrm{n} / \mathrm{a}$ & $\mathrm{n} / \mathrm{a}$ \\
\hline
\end{tabular}

*Note: $\mathrm{E}_{1 / 2}$ is only provided for waves with atleast quasi-reversibility

$* *($ Red $)=$ Reducion $\quad * * *(\mathrm{Ox})=$ Oxidation

Among the four novel radical-conjugated systems (see CV Spectrum 1-4 in Appendices), verdazyl 4 is the only product to exhibit full redox chemical reversibility for the given potential ranges, demonstrated by nearly identical forwards and backwards oxidation and reduction waves. The other three radicals exhibit at least some degree of irreversible chemical processes in the measured potential ranges. Verdazyl $\mathbf{5}$ undergoes reversible one electron reduction at $0.13 \mathrm{v}\left(\mathrm{E}_{1 / 2}=0.37 \mathrm{v}\right)$, but experiences an irreversible reduction at $1.58 \mathrm{v}$. Bisverdazyl 6 on the other hand undergoes a mostly reversible oxidation at-1.20 v $\left(\mathrm{E}_{1 / 2}=0.36 \mathrm{v}\right)$, but undergoes an irreversible oxidation at $0.50 \mathrm{v}\left(\mathrm{E}_{1 / 2}\right.$ $=-1.28 \mathrm{v}$ ), where the corresponding reduction wave appears to double in current output, suggesting a possible two electron reduction processes. Bisverdazyl 7 appears to be the least reversible of the four, only experiencing reversibility for a reduction at $0.43 \mathrm{v}\left(\mathrm{E}_{1 / 2}=\right.$ $0.47 \mathrm{v}$ ), While a non-reversible oxidation takes places at $0.99 \mathrm{v}$ and a non-reversible reduction at $-0.54 \mathrm{v}$, with the corresponding reduction and oxidation waves being absent.

Generally, less reversibility in CV suggests lower analyte stability for redox reactions at the measured potential. This is because the analyte may be undergoing an irreversible chemical reaction while changing redox state in the forward sweep, such that the return 
sweep redox change will not return the analyte to its initial state, as exemplified by Figure 4.12.

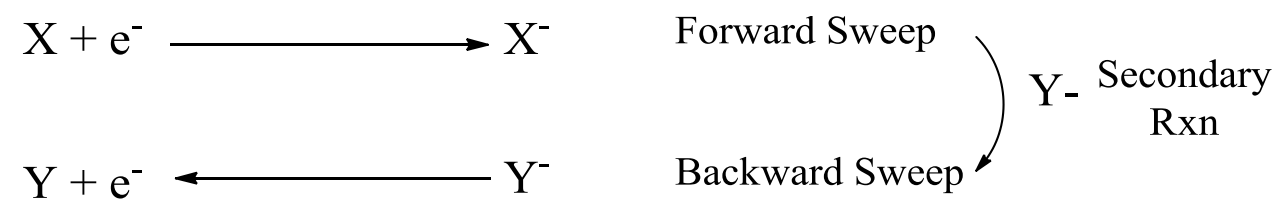

Figure 4.12 Irreversible CV redox scheme

Perhaps the most important take away though is the comparison between the redox potential of the conjugated oligomer and the verdazyl moiety. As previously discussed the conductivity of organic semiconductors is boosted considerably when electrochemically doped to form polaron and bipolaron transportation modes. If the redox potential (band gap) of the verdazyl moiety is smaller than that of the OSC's, it's likely the radical will undergo oxidation before the conjugated chain, preventing the formation of highly conductive doped OSC. This is arguably one of the most important hurdles preventing our synthesized diradicals from realizing their role as conductive spin transport materials. We will discuss possible solutions to this matter in the future works section.

\subsection{Diradical Physical Properties}

\subsubsection{Magnetic Susceptibility}

Magnetic susceptibility is a measure of how strongly a substance interacts with the magnetic field, and provides a quantitative indicator of a substance's magnetic behavior. Magnetic susceptibility measurements of the bisverdazyls not only informs us about the 
type of magnetism present in the substances, it also gives clues about how the two radicals interact with one another (singlet, triplet, or non interacting).

If the magnetic susceptibility $(\chi)$ for a substance is negative, the substance is diamagnetic, and its interaction with the magnetic field causes a repulsive force. It's worth noting that all substances exhibit some degree of diamagnetism due to their paired electrons, but the overall magnetic susceptibility of non-diamagnetic materials is dominated by the larger susceptibility of other forms of magnetism. Small, but positive magnetic susceptibility with adherence to curie's law indicates paramagnetism, and such substances experience weak attraction to magnetic fields.

Figure 4.13 and 4.14 show the plot of magnetic susceptibility vs $T$ and $1 / T$ respectively, for bisverdazyl 6 . Both figures display characteristic magnetic susceptibility of a paramagnetic material, following Curie's law. The slope of the Figure 4.14 graph gives the Curie constant (C), which was found to be $0.68\left(\mathrm{~cm}^{3} . \mathrm{K} / \mathrm{mol}\right)$. This Curie constant value suggests that the two radicals of bisverdazyl $\mathbf{6}$ either do not interact or interact rather weakly, as the value is relatively close to $0.75 \mathrm{~cm}^{3} . \mathrm{K} / \mathrm{mol}$, the Curie constant for non interacting radicals. The lack of diradical interaction in bisverdazyl $\mathbf{6}$ is likely due to the quaterthiophene spacer. A shorter spacer would likely lead to stronger interaction between the diradicals, which we may consider exploring in future studies. 


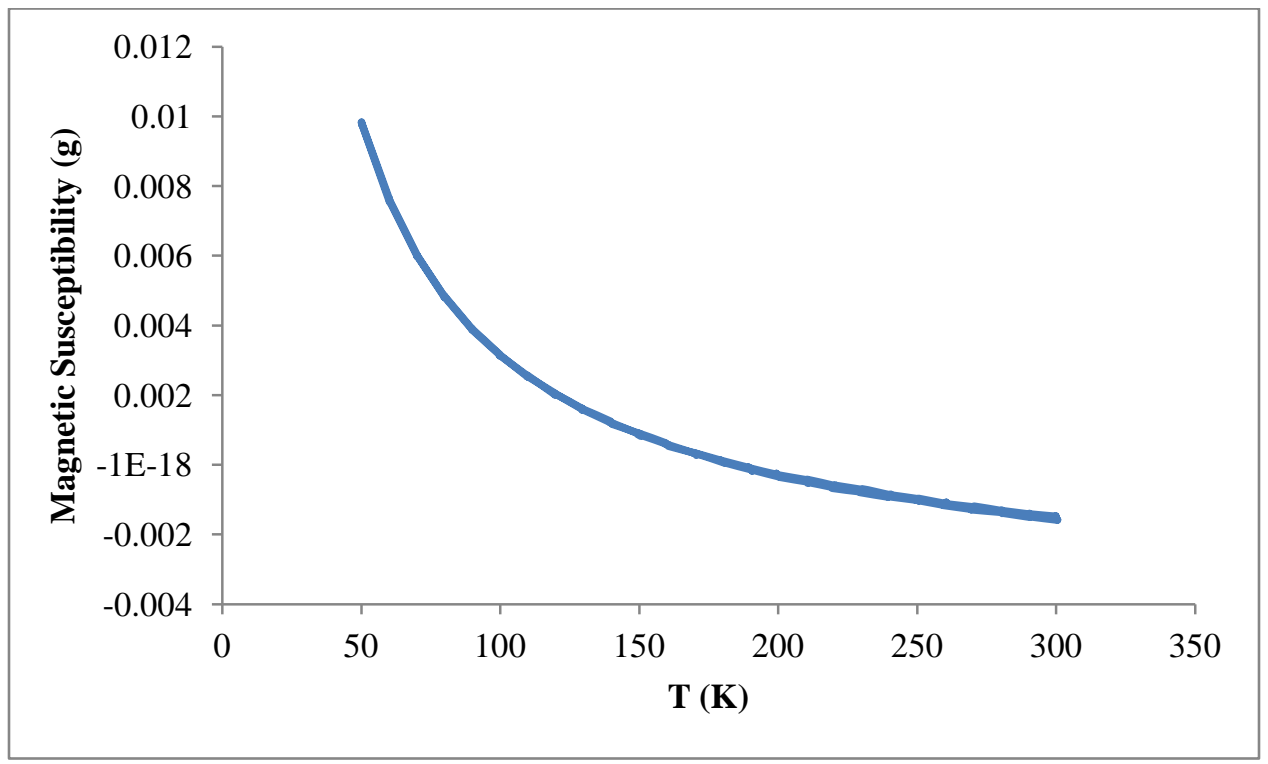

Figure 4.13 $\chi$ vs T Bis (1, 5-diisopropyl-6-oxoverdazyl)-3,3'- $\alpha$ quaterthiophene (6).

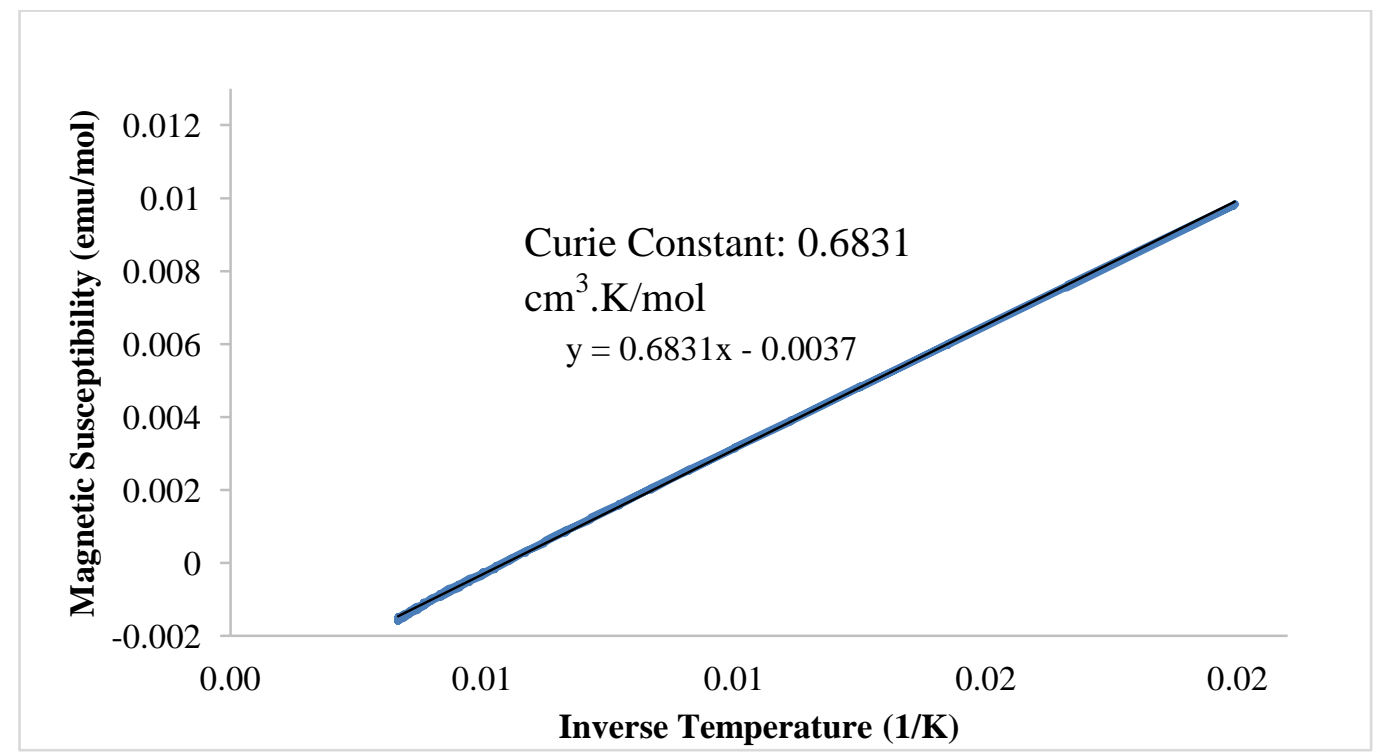

Figure 4.14 $\chi$ vs 1/T Bis (1, 5-diisopropyl-6-oxoverdazyl)-3,3'- $\alpha$ quaterthiophene (6).

The magnetic susceptibility of bisverdazyl 7 (Figure $4.15 \& 4.16$ ) is quite similar to bisverdazyl 6, with characteristic paramagnetic behavior that fits Curie's law. Bisverdazyl 
7 has a smaller Curie constant at $0.56 \mathrm{~cm}^{3} . \mathrm{K} / \mathrm{mol}$, likely due to bisverdazyl 7 sample being less pure than the bisverdazyl 6 sample used for these measurements.

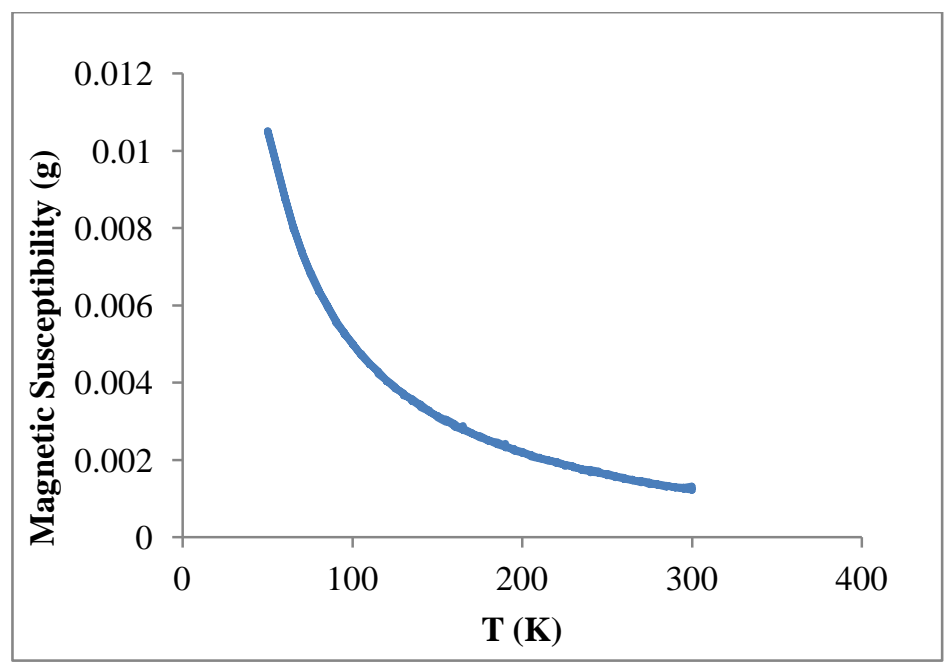

Figure 4.15 $\chi$ vs T Bis(1,5-diisopropyl-6-oxoverdazyl)-3-3'-bithiophene4,4'-biphenyl (7).

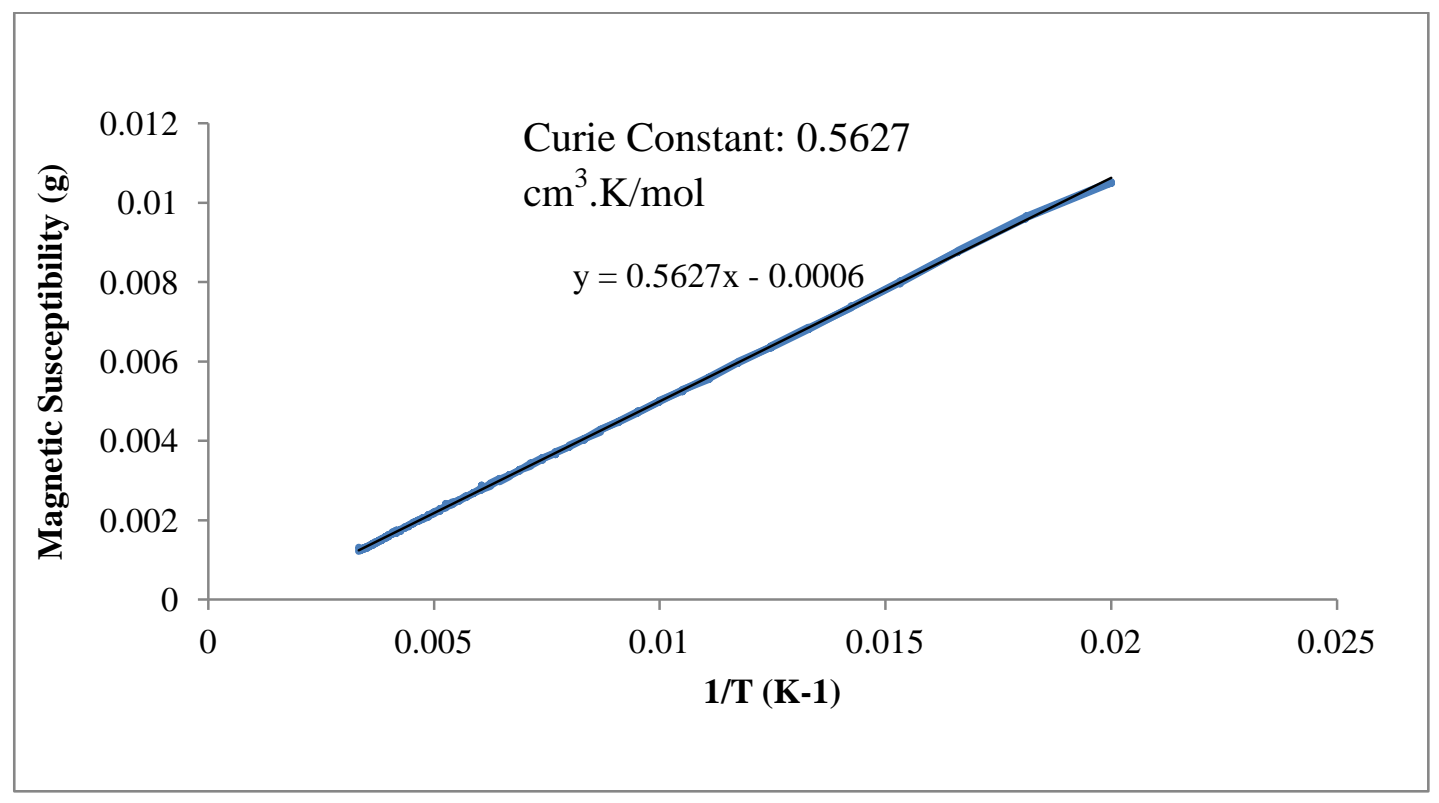

Figure 4.16 $\chi$ vs 1/T Bis(1,5-diisopropyl-6-oxoverdazyl)-3-3'bithiophene-4,4'-biphenyl (7). 


\subsection{Conclusion}

Our primary research goal was to develop bisverdazyl diradicals with oligothiophene spacers for novel physical properties, especially in the context of spintronics. Major hurdles to synthesis included unsubstituted oligothiophene poor solubility, and uncontrolled oligomerization. We succeeded in devising a synthesis strategy to circumvent the above issues by first forming thiophene-verdazyl then proceeding with borylation of the aryl-verdazyl through iridium borylation, the latter step of which is a significant development for the synthesis of future aryl-verdazyl systems that may utilize Suzuki coupling. We were also able to increase the yield of the borylated-thiopheneverdazyl by forming the verdazyl from an alternative route with a previously borylated thiophene-tetrazane. The increased yield allowed us to proceed with double Suzuki coupling reaction of dihalogentated bithiophene and our borylated thiophene verdazyl, to obtain our target molecule bisverdazyl 6 with a thiophene tetramer spacer. The same double Suzuki coupling technique was used with previously developed borylated-phenylverdazyl to obtain bisverdazyl 7.

Several characterization methods were used to verify the structure of novel radical species. UV-VIS, CV and magnetic susceptibility measurements were respectively used to study the electronic, electrochemical and magnetic properties of said radicals. The synthesis strategy developed in this research will allow for the development of other similar conjugated-verdazyls systems. In the future works section we will be discussing a few pending analyses for bisverdazyls 6 \& 7, as well as a particularly promising follow up project that will build on what we have learned in our work here. 


\section{Chapter 5: FUTURE WORK}

The development of fully organic materials with both spin polarizing and conductive properties, was the defining inspiration for our research project. Bisverdazyls with oligothiophene spacers were envisioned to exhibit such properties, and bis $(1,5-$ diisopropyl-6-oxoverdazyl)-3,3'- $\alpha$-quaterthiophene (6) was selected as an initial target towards this goal. We have since successfully synthesized bisverdazyl $\mathbf{6}$, characterized its structure and studied some of its properties through UV-VIS, cyclic voltammetry, and magnetic susceptibility. The analyses most relevant to our overarching goal, conductivity and magnetoresistance have yet to be carried out. Completion of the these analyses will be our foremost goal in future studies.

Even though our study of bisverdazyl 6 has yet to fully conclude, we already suspect conductivity limitations with this diradical due to its relatively short conjugation length. The conductivity prospects are further undermined by cyclic voltammetry data, which suggests that the quaterthiophene chain oxidizes with a smaller or equal potential magnitude to the verdazyl moiety, such that attempts at electrochemically doping the quaterthiophene may instead lead to a change in the oxidation state of the diradicals, and effectively eliminate of the unpaired electrons. This is a serious limitation, as oligothiophenes have modest conductivity unless doped to form polaron and bipolaron charge transport.

We believe we can tackle the above issue by combining oligothiphene with the thiophene derivative, 3,4-ethylenedioxythiophene (EDOT). EDOT (Figure 5.1 for structure) is best known as the monomer of poly(3,4-ethylenedioxythiophene) and poly 
(3,4-ethylenedioxythiophene: polystyrene sulfonate (PEDOT: PSS), which are among the most conductive organic materials, with metal-like conductive properties. ${ }^{45}$ The extraordinary conductivity of the above polymers is due in large part to the considerable electron donor property of EDOT and the many intramolecular interactions (noncovalent) between the oxygens and sulfurs of each monomer, the latter of which is very important in regards to morphology.

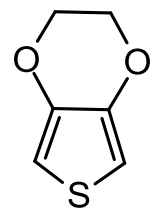

\section{3,4-Ethylene dioxythiophene}

(EDOT)

\section{Figure 5.1 EDOT structure.}

One major disadvantage of oligothiophenes is their poor solubility due to stacking effects and the ridged structure of thiophenes. This was seen with our earlier synthetic work, in which even a thiophene tetramer was poorly soluble. Oligomers consisting of EDOT, on the other hand, fare better in regards to solubility. This is because of the flexible cyclic ether portion of EDOT monomer, which allows the synthesis of soluble unsubstituted oligo-EDOT chains, with as many as 18 EDOT units. ${ }^{46}$ While we were able to form a soluble bisverdazyl with a thiophene tetramer spacer, the diradical was already experiencing some decrease in solubility, such that full dissolution required dichloromethane as solvent. Even a marginally longer thiophene chain could very well lead to a poorly soluble diradical. We believe that by combining EDOT and thiophenes as 
co-oligomers, we can achieve better solubility and synthesize longer conjugated oligomers, with smaller band gaps and redox potentials, increasing overall conductivity.

Turbiez et al. studied various arrangements of mixed thiophene-EDOT co-oligomers, and found the oxygen-sulfur interactions between EDOT and thiophenes lead to important differences in structural conformation (see Figure 5.2).

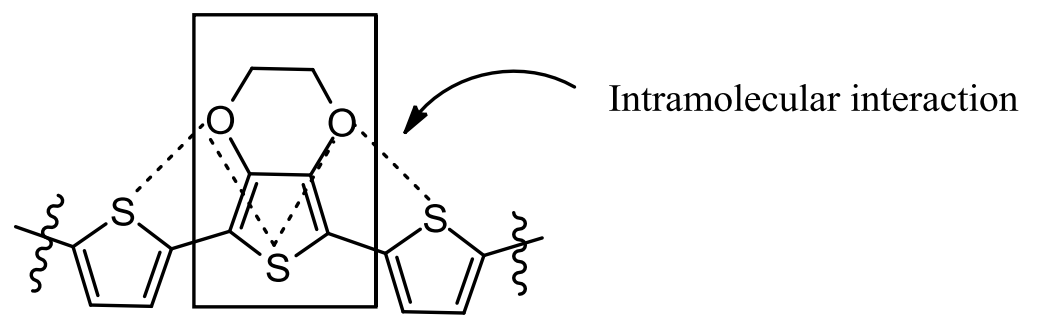

EDOT

Figure 5.2 Intramolecular interactions in thiophene-EDOT oligomers.

Based on Turbiez et al. findings, we believe the best suited thiophene/EDOT arrangement for our purposes would be alternating thiophene-EDOT co-oligomers, as this arrangement was found to lead to planar anti conformation (for the aromatic moiety, as the ether end of EDOT is twisted) between the thiophene and EDOT units, which would maximize the extent of p orbital overlap and improve conjugation. ${ }^{47}$ Alternating thiophene-EDOT oligomers may be preferable to just oligo-EDOT, as EDOT oligomers can often undergo unintentional polymerization, as the alpha position on EDOT is particularly reactive. Furthermore, successive EDOT units imbue the structure with very small oxidation potentials, so that oligo-EDOTs can also undergo spontaneous oxidizations, making it harder to synthesize neutral forms of the conjugated system for more controlled electrochemical doping. ${ }^{46}$ 
The non-covalent oxygen-sulfur interactions of EDOT units extends beyond just intramolecular interactions to intermolecular interactions as well. The high proportion of regular intermolecular interactions leads to more organized planar $\pi$-stacking molecular motifs. ${ }^{43}$ This contrasts with herringbone $\pi$-stacking which we observed to some degree with our novel verdazyl compounds. Planar packing generally increases conduction, as it allows for better intermolecular overlap of energy levels, giving a more band like transport characteristic, while herringbone packing exhibits the more traditional 2D molecular transport mechanism, with more disordered mobility (Figure 5.3). ${ }^{43}$

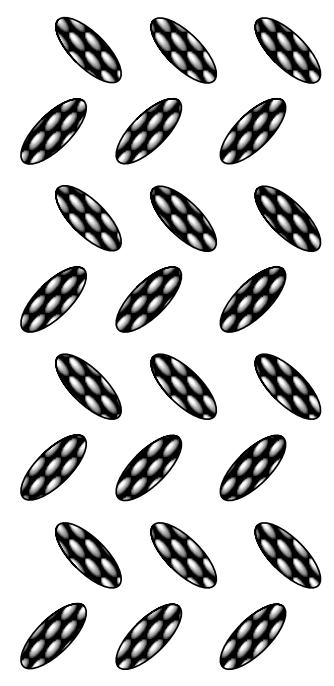

Herringbone $\pi$ Stacking Motive

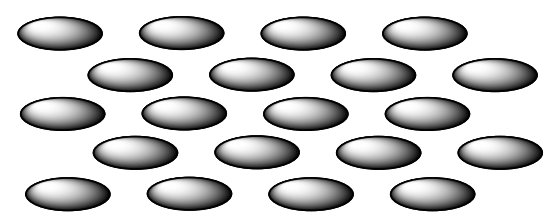

Planar $\pi$-Stacking Motive

Figure 5.3 Molecular packing motifs. 


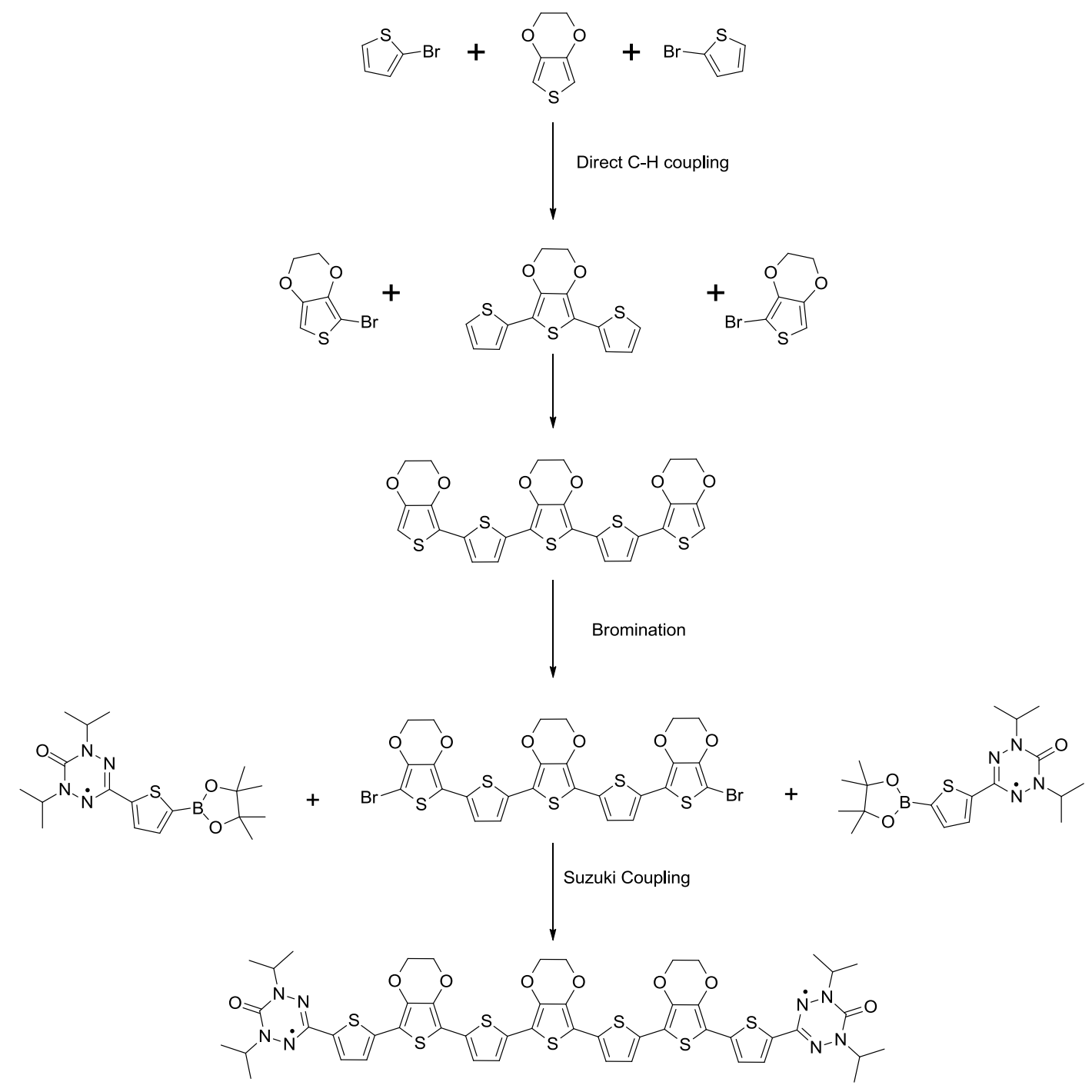

Scheme 5.1 Synthesis plan for mixed thiophene/EDOT co-oligomer Bisverdazyl system.

Scheme 5.1 shows our proposed synthesis route to obtain bisverdazyls with thiophene-EDOT co-oligomer spacers. The direct C-H coupling of halogentated thiophene with terminal EDOTs explored in Liu et al. paper, allows us to elongate the thiophene-EDOT chain with fewer steps and no boronic acid, save for the final reaction step joining the oligomer to the borylated thiophene verdazyl. ${ }^{48}$ We believe the proposed 
synthesis will be a worthwhile follow up to the research presented here, and will build on what has been learned over the course of the project.

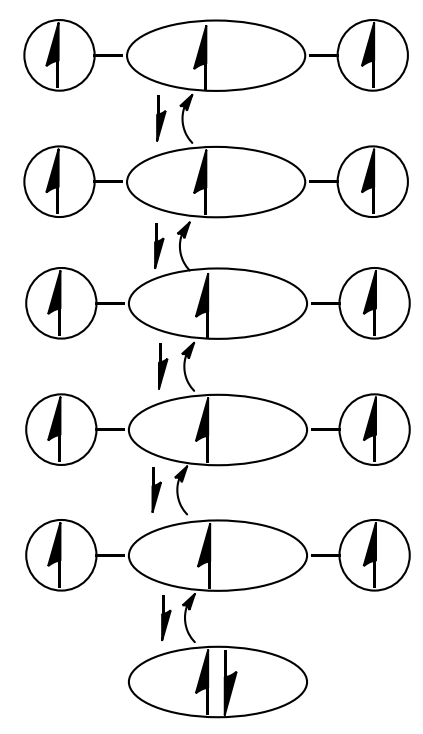

Figure 5.4 Proposed spin propagation mechanism in conductive diradical.

We conclude with Figure 5.4, which provides a simplified and speculative visual representation of how a conductive oligomer-bisverdazyl systems might act as materials for spin transport. 


\section{REFERENCES:}

1. Moore, G. E. P. IEEE. 1998, 86 (1), 82-85.

2. Theis, T. N.; Wong, P. H. S. Comput. Sci. Eng. 2017, 19 (2), 41-50.

3. Peper, F. New. Generat. Comput, 2017, 35 (3), 253-269.

4. Kang, W.; Zhang, Y.; Wang, Z.; Klein, J.-O.; Chappert, C.; Ravelosona, D.; Wang, G.; Zhang, Y.; Zhao, W. ACM. J. Emerg. Tech. Com. 2015, 12 (2), 1-42.

5. Guarnieri, M. Iee. Ind. Electron. M. 2012, 6 (1), 41-43.

6. Mehta, V. K.; Mehta, R. Principles of Electronics; S Chand and Co. Ltd: New Delhi., 2008; pp 28-47

7. Nag, B. R. Indian. J. Radio. Space. 1990, 19, 306-308

8. Weller, P. F. J. Chem. Educ. 1967, 44 (7), 391-393.

9. Juster, N. J. J. Chem. Educ. 1963, 40 (10), 547-555.

10. Coropceanu, V.; Li, H.; Winget, P.; Zhu, L.; Brédas, J.-L. Annu. Rev. Mater. Res. 2013, 43 (1), pp 63-87.

11. Kertesz, M.; Choi, C. H.; Yang, S. Chem. Rev. 2006, 105 (10), 3448-3481.

12. Dechun, Z. in Organic Light-Emitting Diodes (OLED); Buckley, A., Ed.; Materials, Devices and Applications: Cambridge, U.K., 2013; pp 114-142.

13. Gregg, B. A.; Hanna, M. C. J. Appl. Phys. 2003, 93 (6), 3605-3614.

14. Olivier, Y.; Lemaur, V.; Brédas, J. L.; Cornil, J. J. Phys. Chem. A. 2006, 110 (19), 6356-6364.

15. Schott, S.; Mcnellis, E. R.; Nielsen, C. B.; Chen, H.-Y.; Watanabe, S.; Tanaka, H.; Mcculloch, I.; Takimiya, K.; Sinova, J.; Sirringhaus, H. Nat. Commun. 2017, 8, $1-10$.

16. Harris IR, Williams AJ. Magnetic materials. In: Rawlings RD, editor. Material science and engineering., 2009, 2, 49-84.

17. Kolhatkar, A.; Jamison, A.; Litvinov, D.; Willson, R.; Lee, T. Int. J. Mol. Sci. 2013, 14(8), 15977-16009 
18. Gu, H.; Zhang, X.; Wei, H.; Huang, Y.; Wei, S.; Guo, Z. Chem. Soc. Rev. 2013, 42 (13), 5907-5943

19. Turro, N. J. Modern Molecular Photochemistry; University Science Books: Sausalito, CA., 1991.

20. Joshi, V. K. Eng. Sci. Technol. Int. J. 2016, 19 (3), 1503-1513.

21. Zhang, Y.; Zhao, W.; Klein, J.-O.; Kang, W.; Querlioz, D.; Zhang, Y.; Ravelosona, D.; Chappert, C. Des. Aut. Test. Europe. 2014, 1-6.

22. Boona, S. R.; Myers, R. C.; Heremans, J. P. Energ. Environ. Sci. 2014, 7 (3), 885-910

23. Togo, H.; Togo, H. In Advanced Free Radical Reactions for Organic Synthesis; Elsevier: Amsterdam, Netherlands, 2004; pp 1-37.

24. Bačić, G.; Spasojević, I.; Šećerov, B.; Mojović, M. Spectrochim. Acta. Mol. Biomol. Spectrosc. 2008, 69 (5), 1354-1366.

25. Vostrikova, K. E. Coordin. Chem. Rev. 2008, 252 (12-14), 1409-1419.

26. Blundell, S. J.; Pratt, F. L. J. Phys.: Condens. Matter. 2004, 16, 771-828.

27. Hicks, R. G.; Lemaire, M. T.; Thompson, L. K.; Barclay, T. M. J. Am. Chem. Soc. 2000, 122 (33), 8077-8078.

28. Barclay, T. M.; Hicks, R. G.; Lemaire, M. T.; Thompson, L. K. Chem. Comm. 2000, No. 21, 2141-2142.

29. Brook, D. J. R. Comment. Inorg. Chem, 2014, 35 (1), 1-17.

30. Le, T.-N.; Trevisan, T.; Lieu, E.; Brook, D. J. R. Eur. J. Org. Chem., 2017, 2017 (7), $1125-1131$.

31. Pare, E.C.; Brook, D. J. R.; Brieger, A.; Badik, M.; Schinke, M. Org. Biomol. Chem. 2005, 3, 4258-4261

32. Tour, J. M.; Lamba, J. J. S. J. Am. Chem. Soc. 1993, 115 (11), 4935-4936.

33. Szajda, M.; Lam, J. N. In Comprehensive Heterocyclic Chemistry II. a Review of the Literature, 1982-1995: The Structure, Reactions, Synthesis and Uses of Heterocyclic Compounds; Pergamon: Oxford, 1996; Vol. 2, pp 437-490. 
34. Zhang, L.; Colella, N. S.; Cherniawski, B. P.; Mannsfeld, S. C. B.; Briseno, A. L. Oligothiophene Semiconductors: Synthesis, Characterization, and Applications for Organic Devices. ACS Appl. Mater. Interfaces. 2014, 6 (8), 5327-5343.

35. Musick, K. Y.; Hu, Q.-S.; Pu, L. Synthesis of Binaphthyl-Oligothiophene Copolymers with Emissions of Different Colors: Systematically Tuning the Photoluminescence of Conjugated Polymers. Macromolecules. 1998, 31 (9), 2933-2942.

36. Ishiyama, T.; Murata, M.; Miyaura, N. Palladium(0)-Catalyzed Cross-Coupling Reaction of Alkoxydiboron with Haloarenes: A Direct Procedure for Arylboronic Esters. J. Org. Chem. 1995, 60, 7508-7510.

37. Lennox, A. J. J.; Lloyd-Jones, G. C. Selection of Boron Reagents for Suzuki-Miyaura Coupling. Chem. Soc. Rev. 2014, 43, 412-443.

38. Song, J.; Wei, F.; Sun, W.; Cao, X.; Liu, C.; Xie, L.; Huang, W. Highly Efficient C-C Cross-Coupling for Installing Thiophene Rings into $\pi$-Conjugated Systems. Org. Chem. Front. 2014, 1, 817-820.

39. Singh, Y. Electrical Resistivity Measurements: A Review. Int. J. Mod. Phys. 2013, 22, 745-756.

40. Hwang, E.; Lusker, K. L.; Garno, J. C.; Losovyj, Y.; Nesterov, E. E. Semiconducting Polymer Thin Films by Surface-Confined Stepwise Click Polymerization. Chem. Commun. 2011, 47(43), 11990-11992.

41. Tajuddin, H.; Harrisson, P.; Bitterlich, B.; Collings, J. C.; Sim, N.; Batsanov, A. S.; Cheung, M. S.; Kawamorita, S.; Maxwell, A. C.; Shukla, L.; Morris, J.; Lin, Z.; Marder, T. B.; Steel, P. G. Iridium-Catalyzed C-H Borylation of Quinolines and Unsymmetrical 1,2-Disubstituted Benzenes: Insights into Steric and Electronic Effects on Selectivity. Chem. Sci, 2012, 3 (12), 3505-3515.

42. Ishiyama, T.; Takagi, J.; Yonekawa, Y.; Hartwig, J. F.; Miyaura, N. Iridium-Catalyzed Direct Borylation of Five-Membered Heteroarenes by Bis(Pinacolato)Diboron: Regioselective, Stoichiometric, and Room Temperature Reactions. Adv. Synth. Catal. 2003, 354, 1103-1106.

43. Filo, J.; Putala, M. Semiconducting Organic Molecular Materials. J. Electr. Eng. 2010, 61(5), 314-320

44. Kolanji, K.; Ravat, P.; Bogomyakov, A. S.; Ovcharenko, V. I.; Schollmeyer, D.; Baumgarten, M. Mixed Phenyl and Thiophene Oligomers for Bridging Nitronyl Nitroxides. The J. Org. Chem. 2017, 82 (15), 7764-7773. 
45. Groenendaal, L.; Jonas, F.; Freitag, D.; Pielartzik, H.; Reynolds, J. R. Poly(3,4Ethylenedioxythiophene) and Its Derivatives: Past, Present, and Future. Adv. Mater. 2000, 12 (7), 481-494.

46. Dai, Q.; Li, Y.; Zhai, L.; Sun, W. 3,4-Ethylenedioxythiophene (EDOT)-Based $\pi$ Conjugated Oligomers: Facile Synthesis and Excited-State Properties. J. Photoch. Photobio. A., 2009, 206 (2-3), 164-168.

47. Turbiez, M.; Frère, P.; Allain, M.; Videlot, C.; Ackermann, J.; Roncali, J. Design of Organic Semiconductors: Tuning the Electronic Properties of $\pi$-Conjugated Oligothiophenes with the 3,4-Ethylenedioxythiophene (EDOT) Building Block. Chem. Eur. J. 2005, 11 (12), 3742-3752.

48. Liu, C.-Y.; Zhao, H.; Yu, H.-H. Efficient Synthesis of 3,4-Ethylenedioxythiophene (EDOT)-Based Functional $\pi$-Conjugated Molecules through Direct $\mathrm{C}-\mathrm{H}$ Bond Arylations. Org. Lett. 2011, 13 (15), 4068-4071.

49. Macrae, C. F.; Edgington, P. R.; McCabe, P.; Pidcock, E.; Shields, G. P.; Taylor, R.; Towler, M.; Van de Streek, J. J. Appl. Cryst. 39, 453-457, 2006. 


\section{APPENDICES}

NMR Spectra 
H-NMR 1: 2,4-Diisopropyl-6-thiophene-

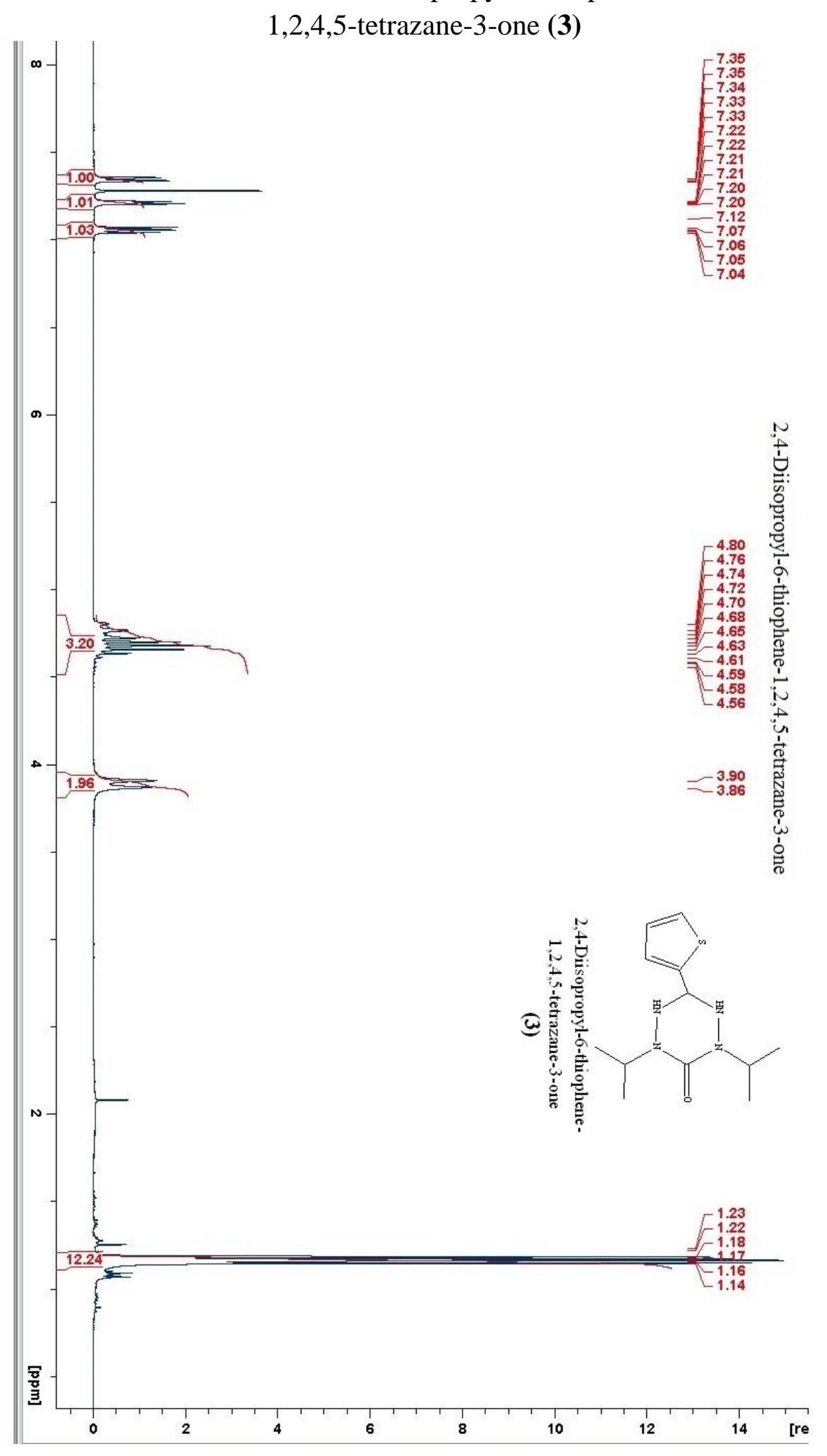


C-NMR 1: 2,4-Diisopropyl-6-thiophene-

1,2,4,5-tetrazane-3-one (3)

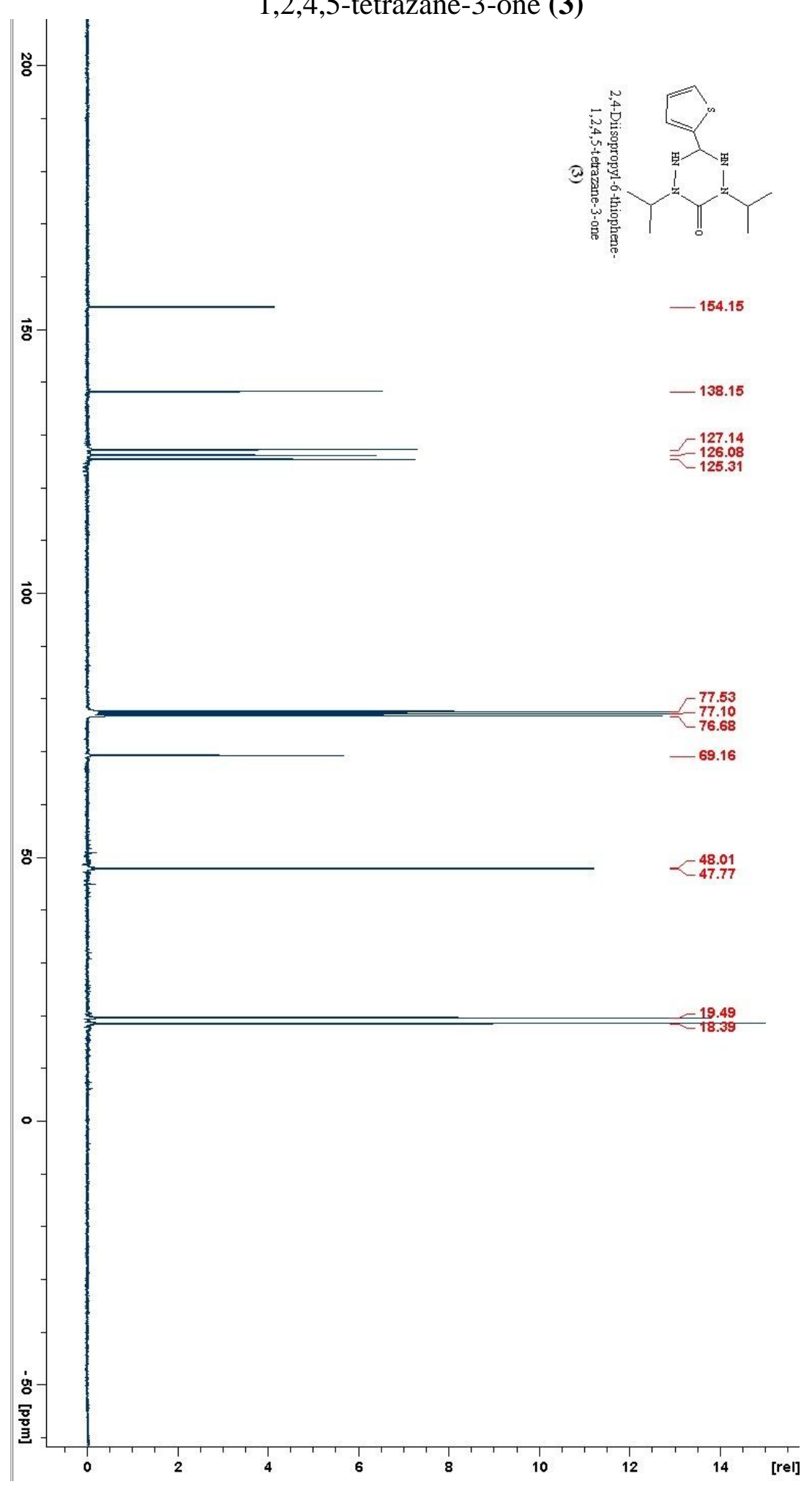


H-NMR 2: Synthesis of 5-Formyl-2-thienylboronic acid pinacol ester (3')

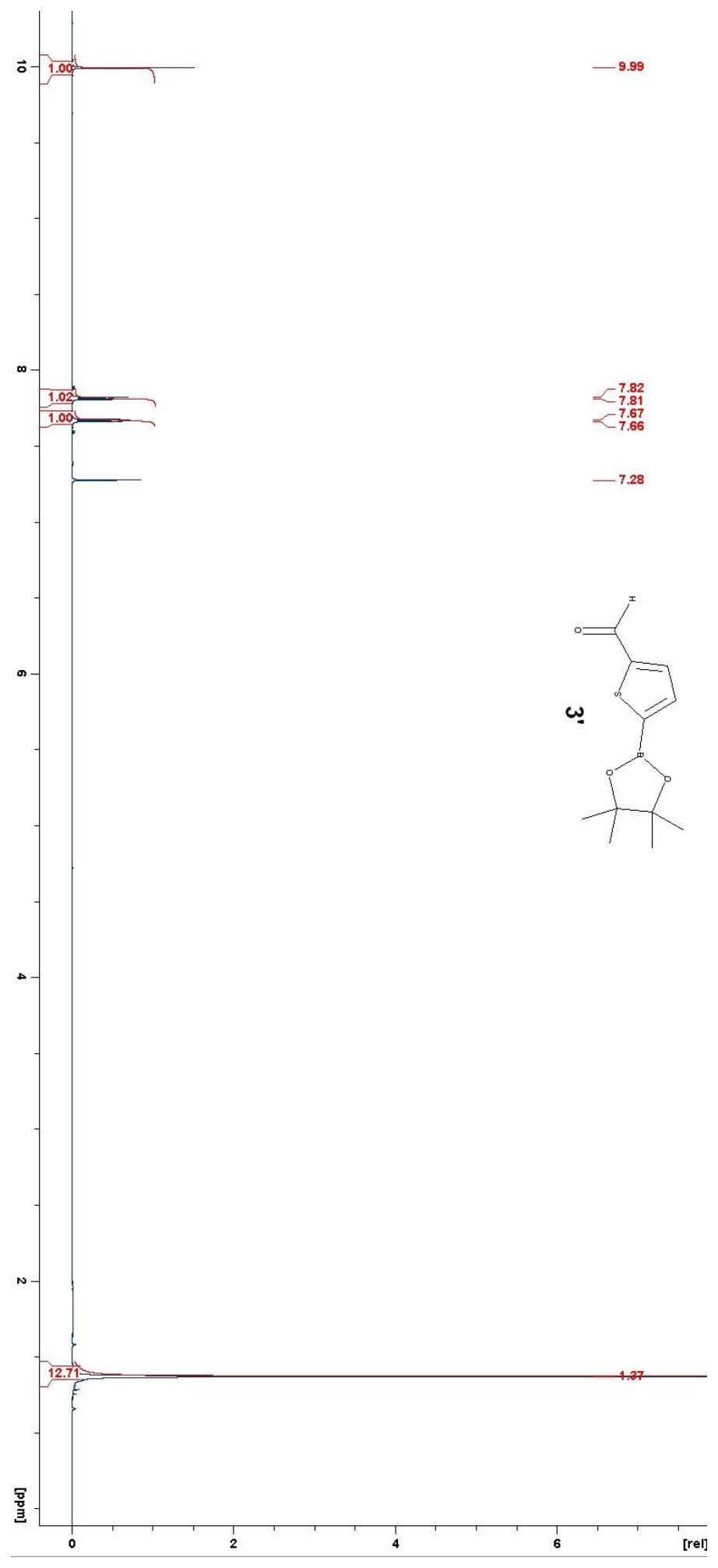


H-NMR 3: 2,4-Diisopropyl-6-thiophene-5-boronic acid pinacol ester-1,2,4,5-tetrazane-3-one (4')

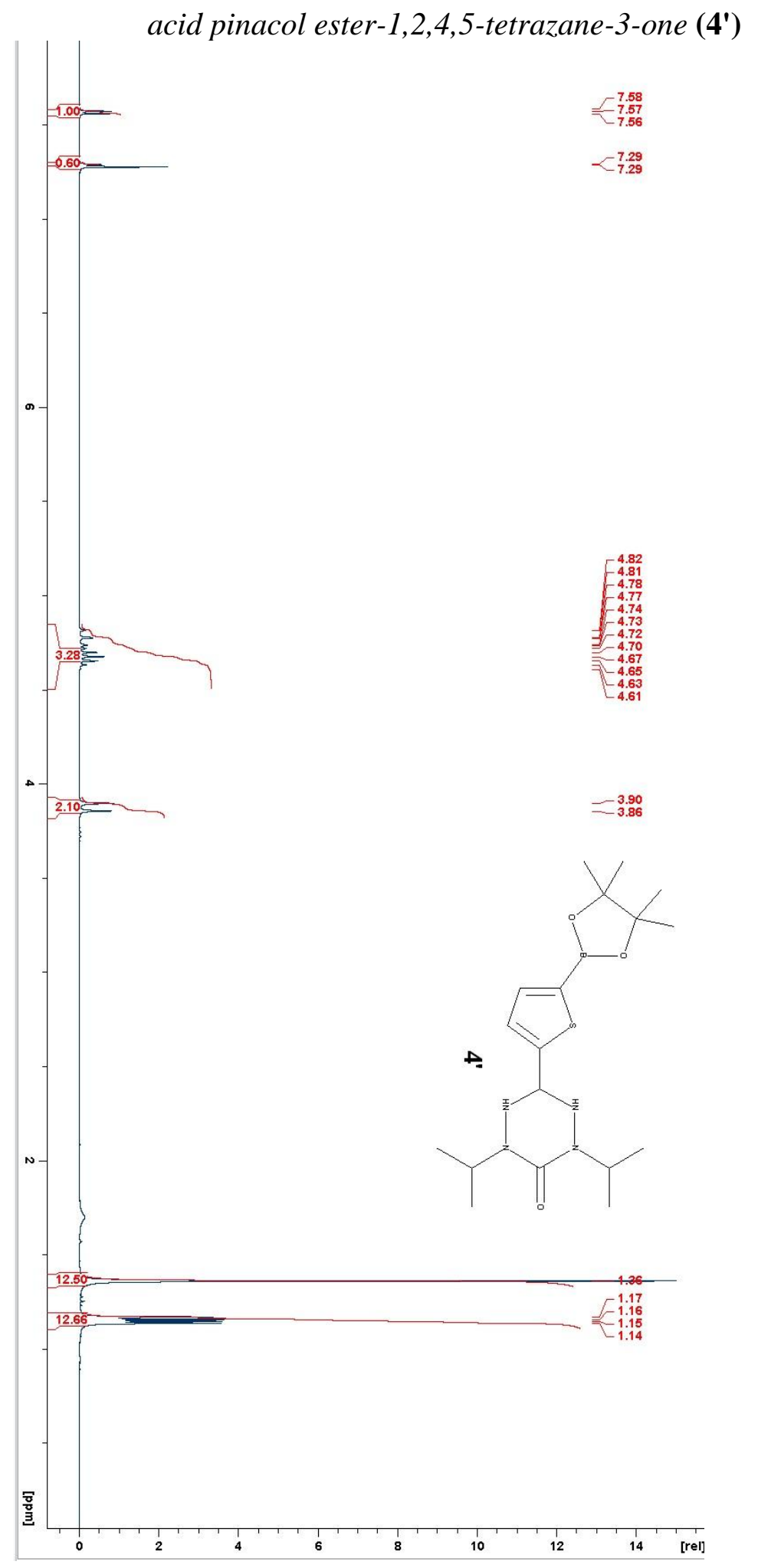


C-NMR 2: 2,4-Diisopropyl-6-thiophene-5-boronic acid pinacol ester-1,2,4,5-tetrazane-3-one (4')

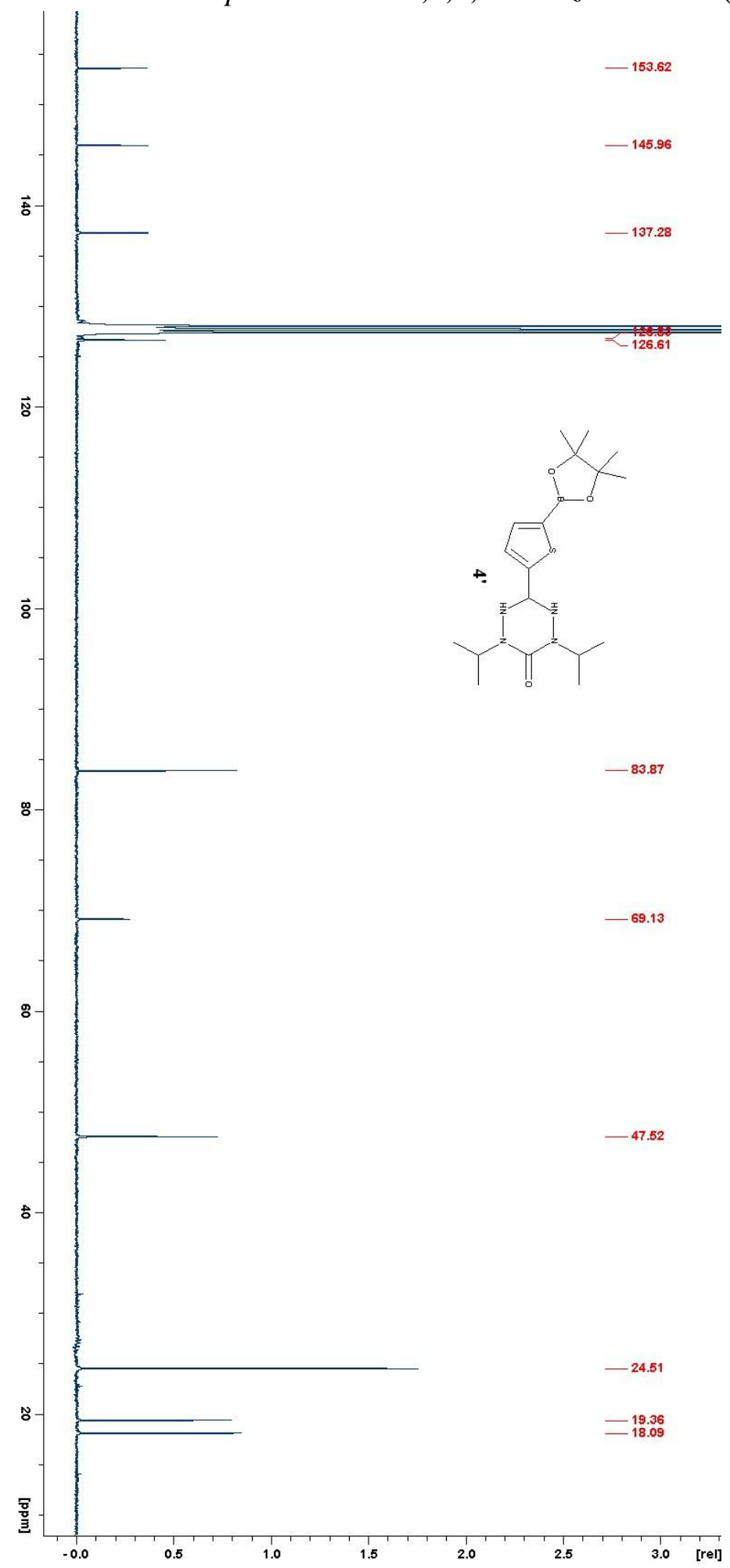




\section{UV-VIS}

UV-VIS Spectrum 1: 1,5-Diisopropyl-3-thiophene-6-oxoverdazyl (4)

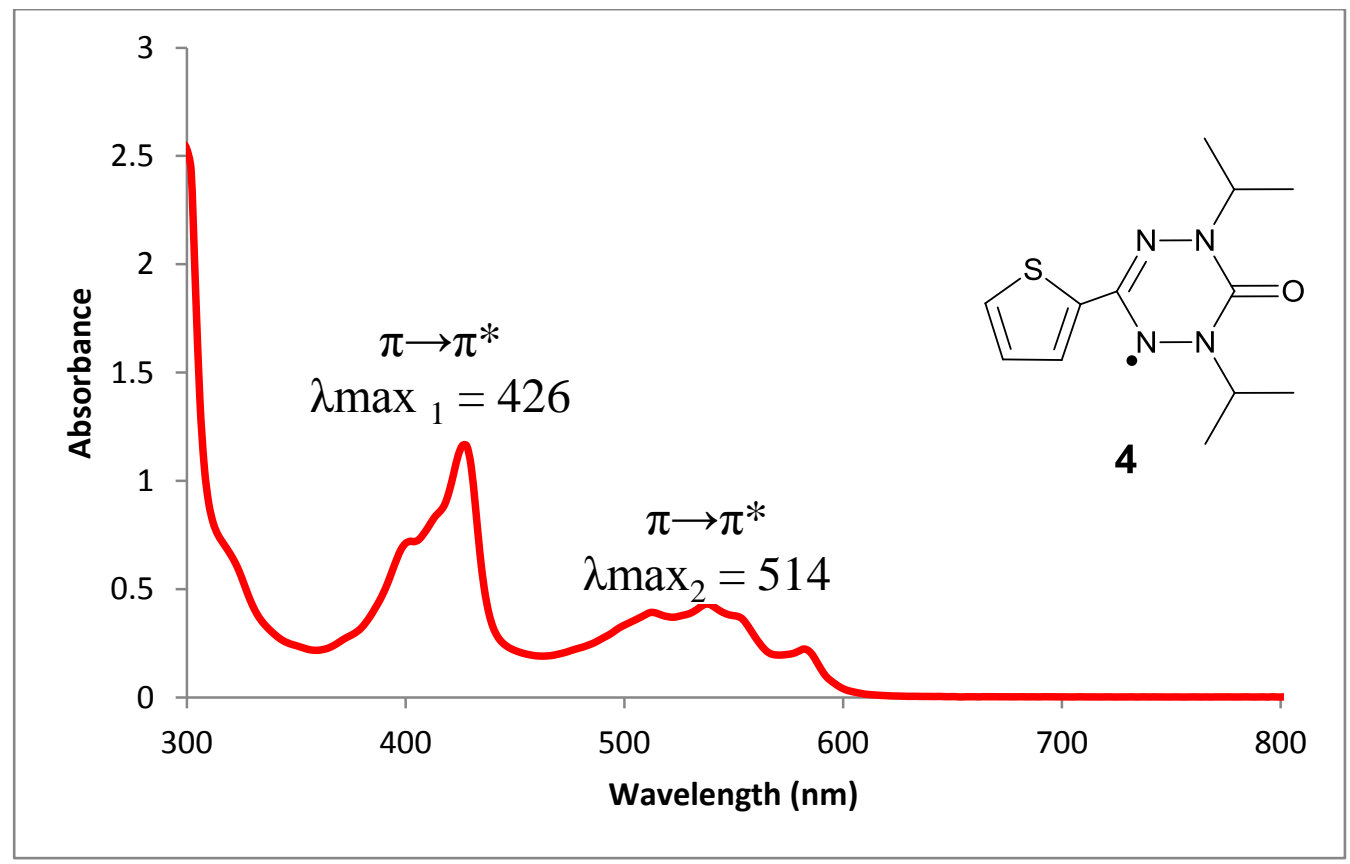

UV-VIS Spectrum 2: 1,5-Diisopropyl-3-thiophene-5-boronic acid pinacol ester-6oxoverdazyl (5)

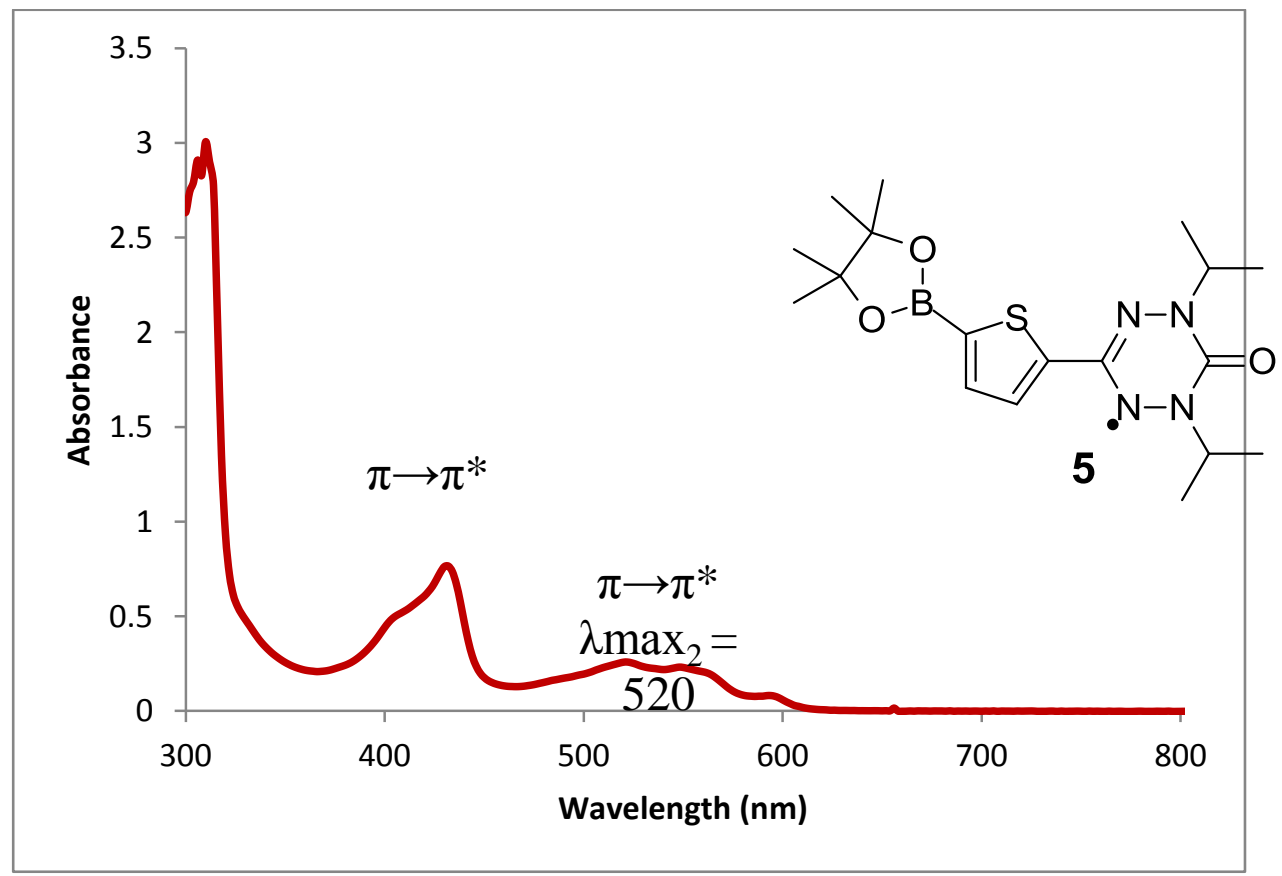


UV-VIS Spectrum 3: Bis (1, 5-diisopropyl-6-oxoverdazyl)-3,3'- $\alpha$-quaterthiophene (6)

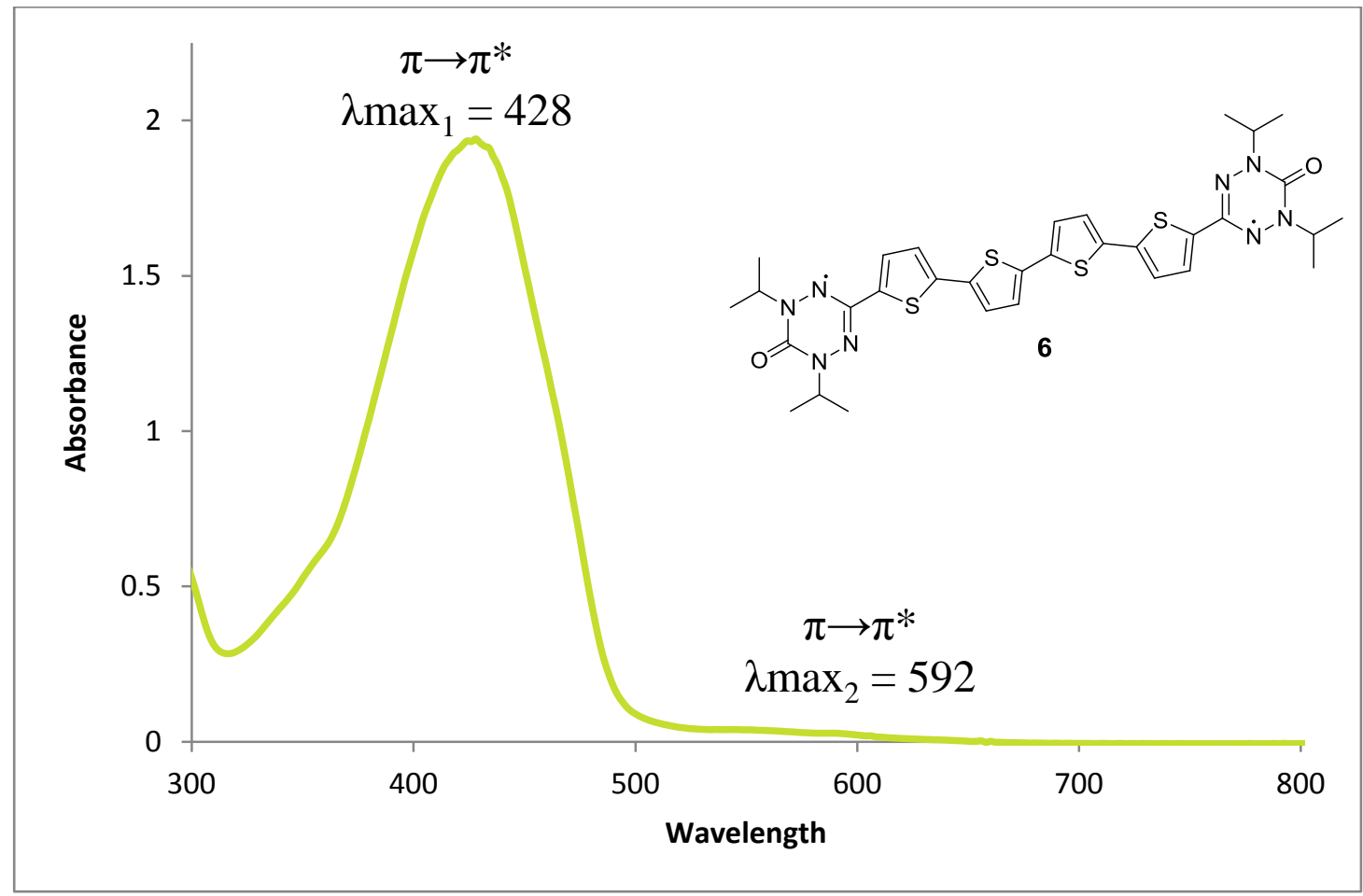

UV-VIS Spectrum 4: Bis (1, 5-diisopropyl-6-oxoverdazyl)-3,3'-biphenyl-4,4'bithiophene (7)

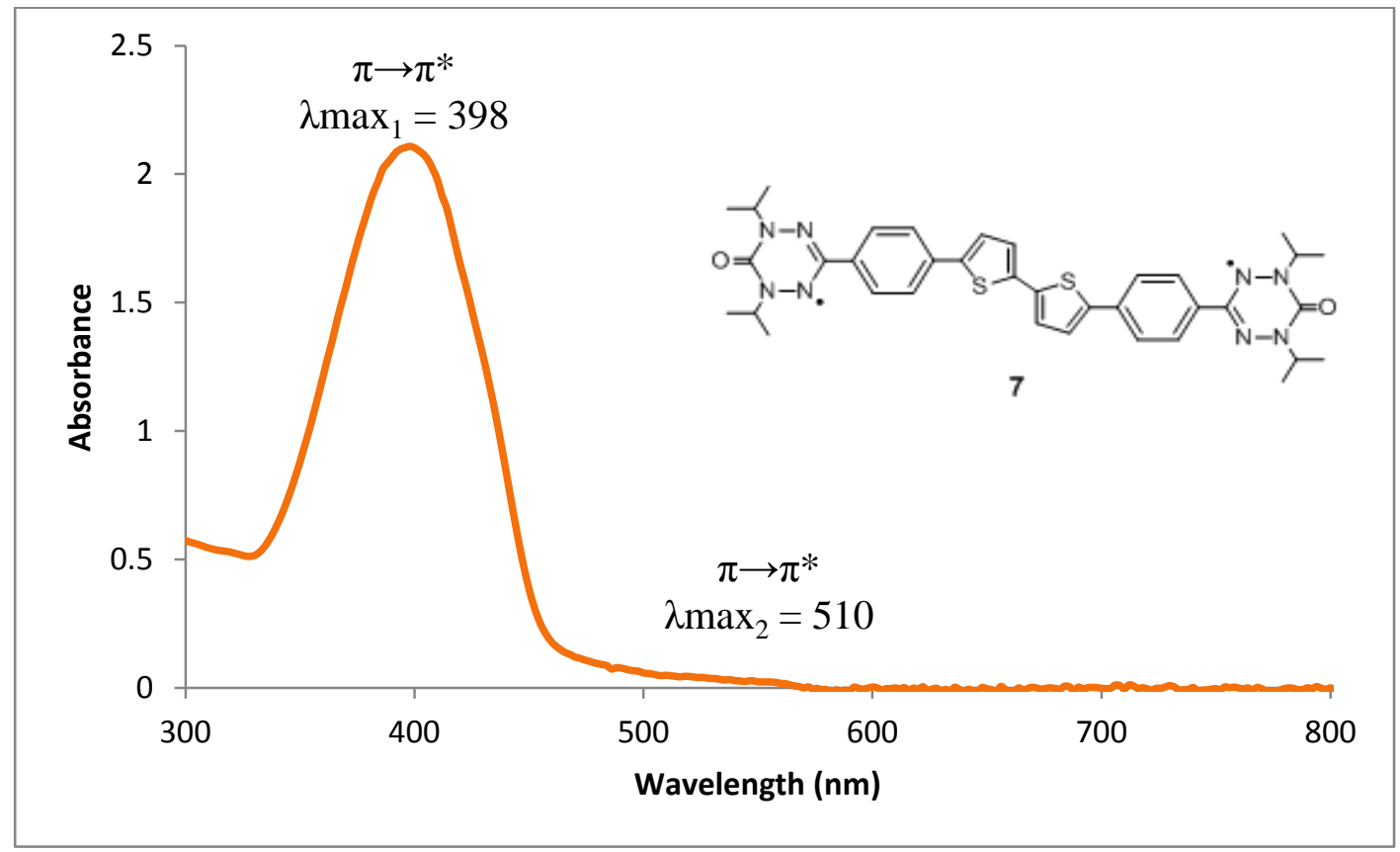




\section{Mass Spectroscopy}

GC-MS 1: 2,4-Diisopropyl-6-thiophene-1,2,4,5-tetrazane-3-one (3)

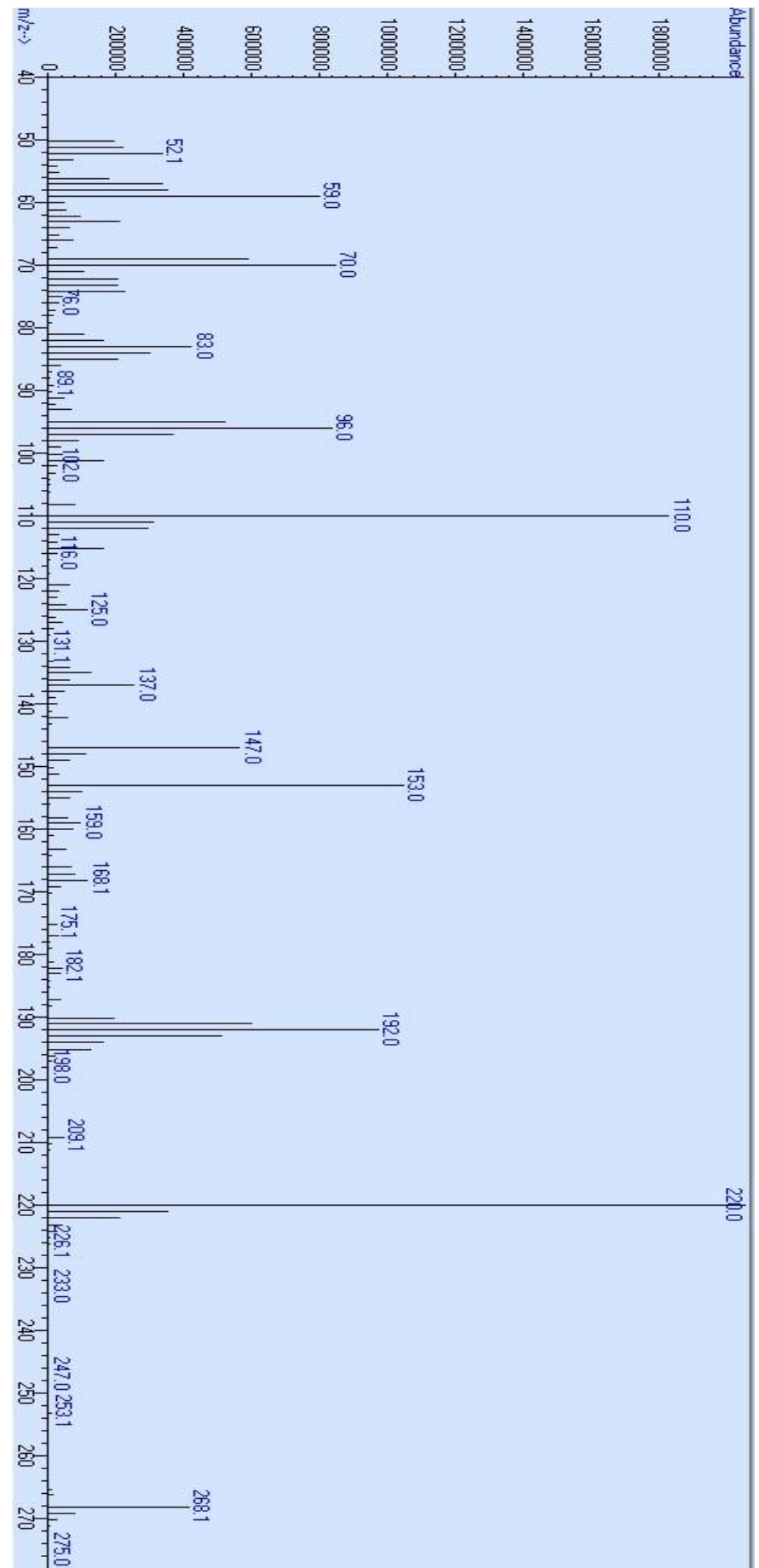


GC-MS 2 : 1,5-Diisopropyl-3-thiophene-6-oxoverdazyl (4)

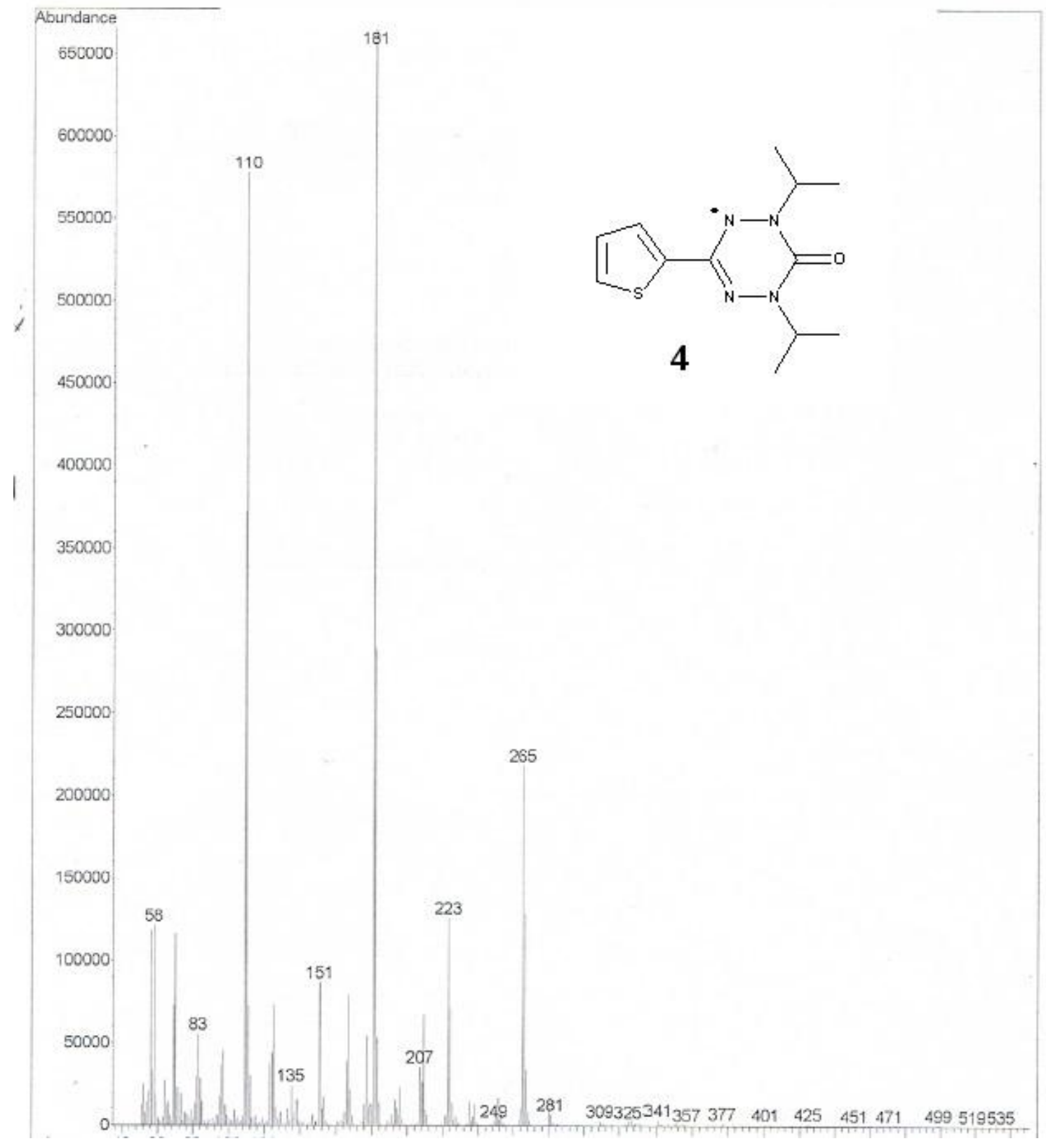


GC-MS 3: 1,5-Diisopropyl-3-thiophene-5-boronic acid pinacol ester-6oxoverdazyl (5)

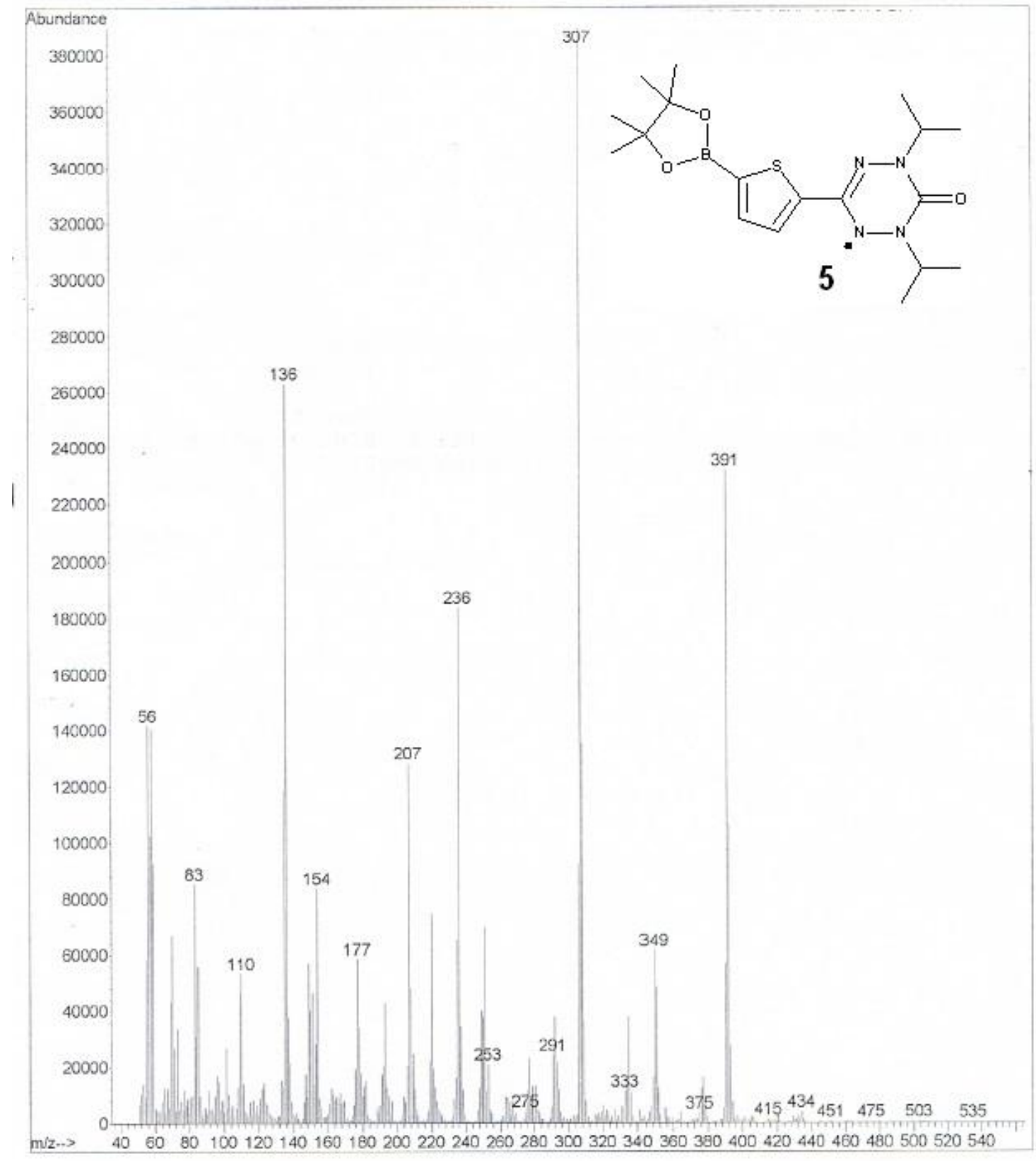


GC-MS 4: 5-Formyl-2-thienylboronic acid pinacol ester (3')

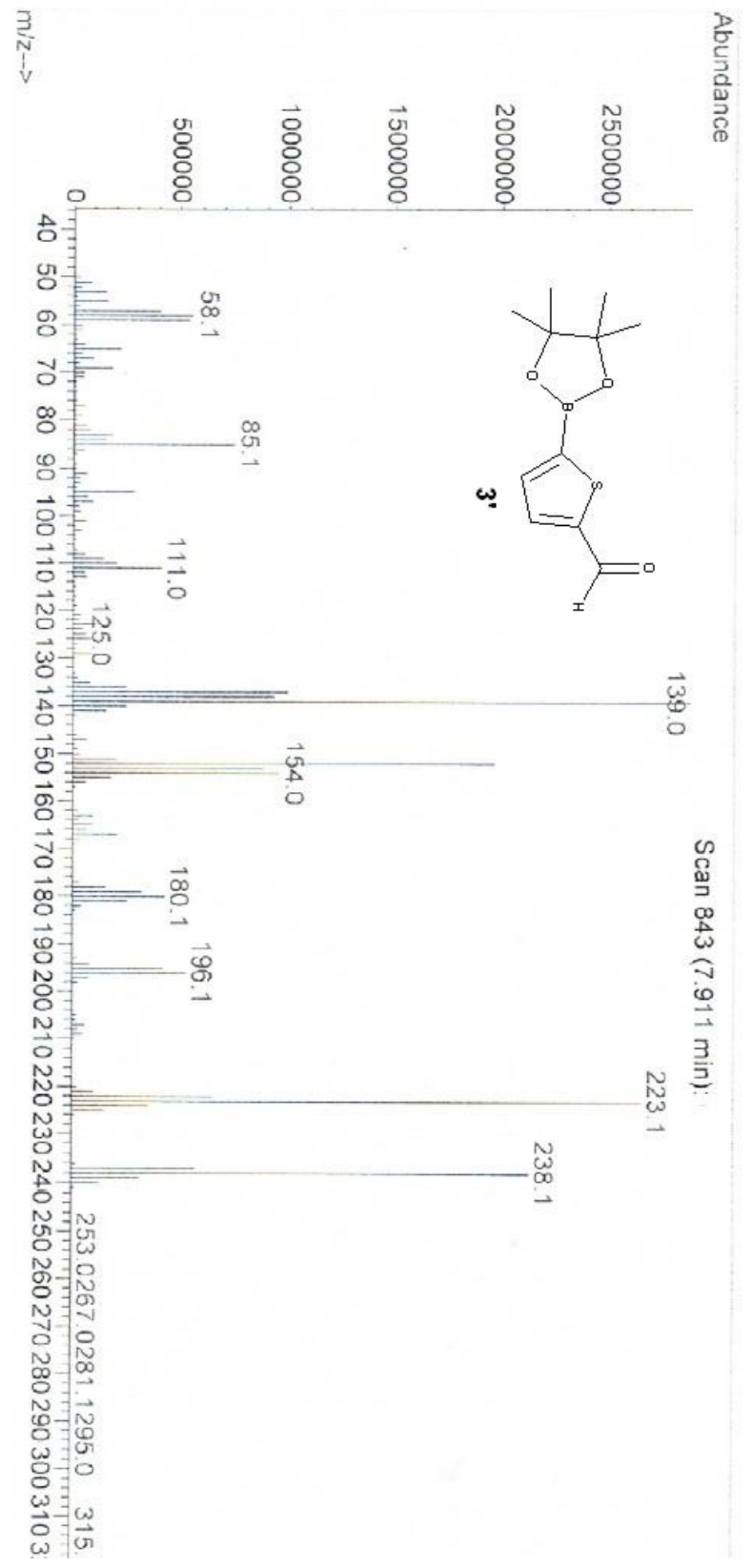


GC-MS 5: 2,4-Diisopropyl-6-thiophene-5-boronic acid pinacol ester1,2,4,5-tetrazane-3-one (4')

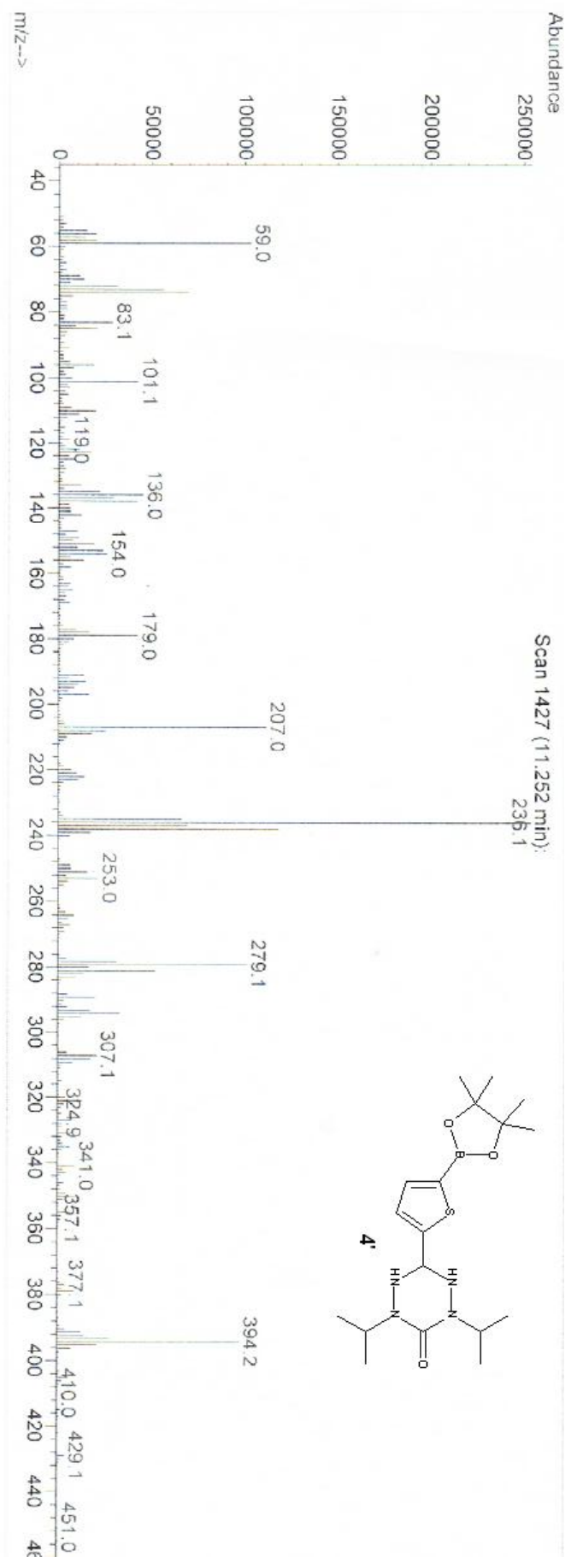


LC-MS 1: Bis (1, 5-diisopropyl-6-oxoverdazyl)-3,3'- $\alpha$-quaterthiophene (6)

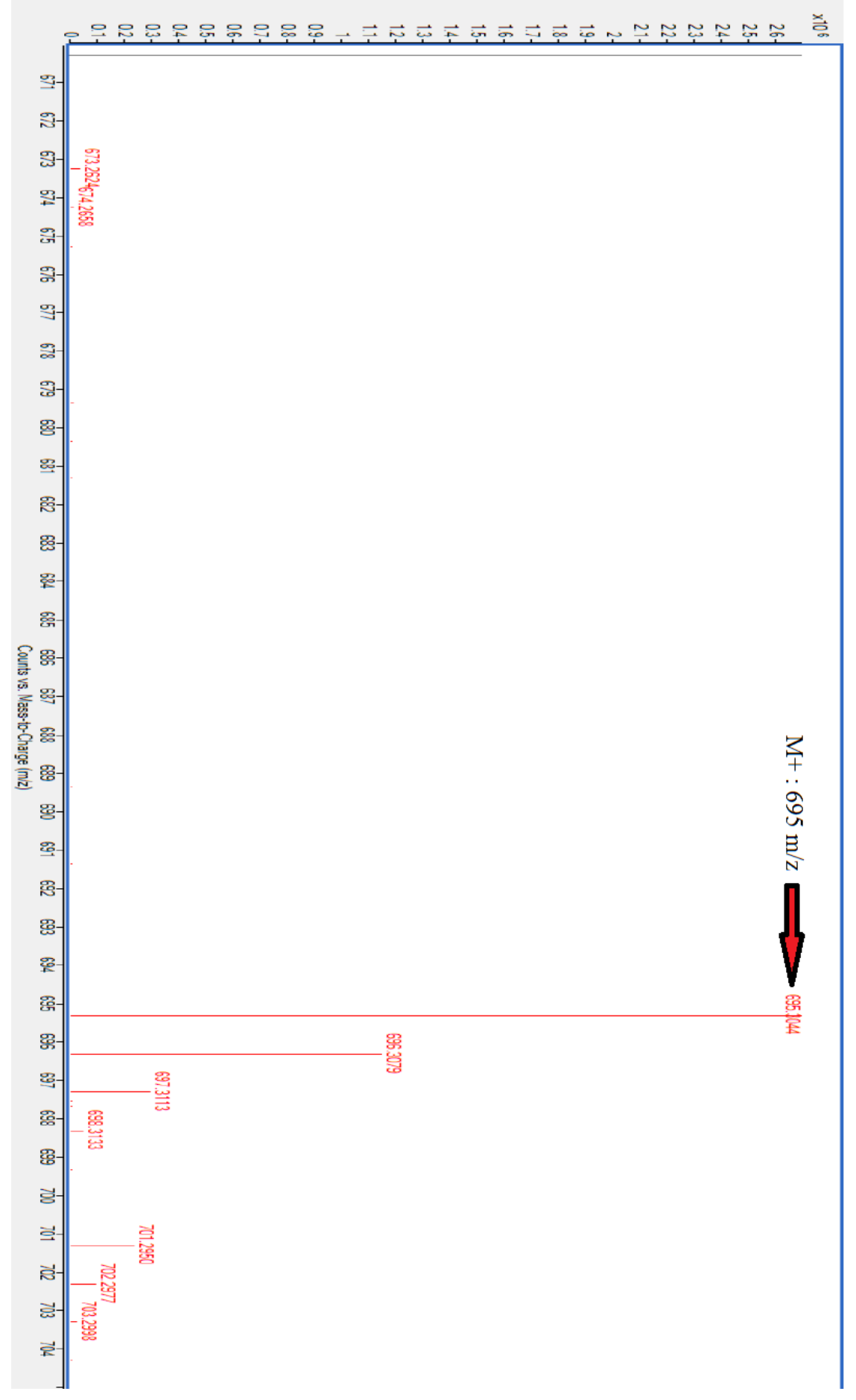




\section{Cyclic Voltammetry (CV)}

CV Spectrum 1: 1,5-Diisopropyl-3-thiophene-6-oxoverdazyl (4)

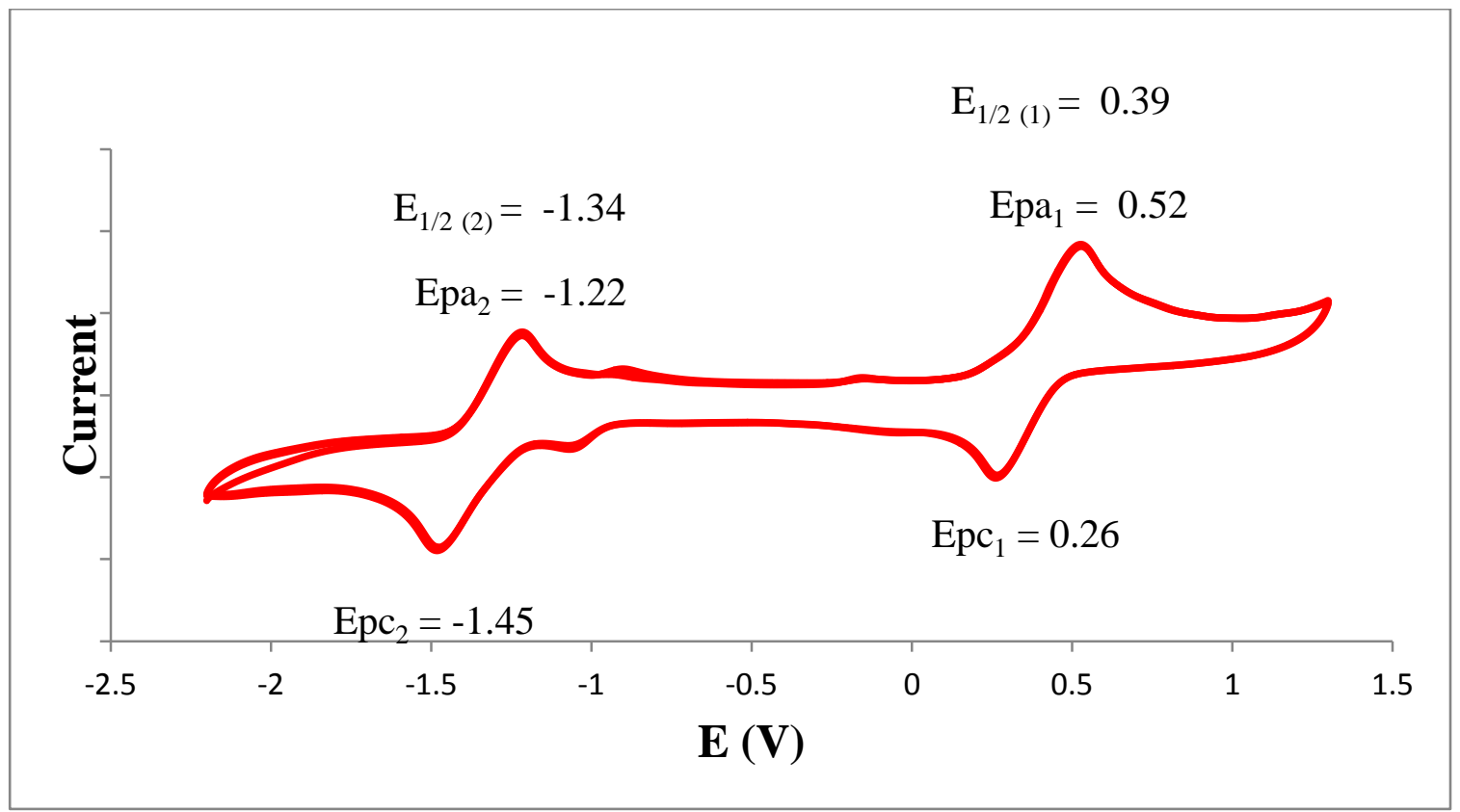

CV Spectrum 2: 1,5-Diisopropyl-3-thiophene-5-boronic acid pinacol ester-6oxoverdazyl (5)

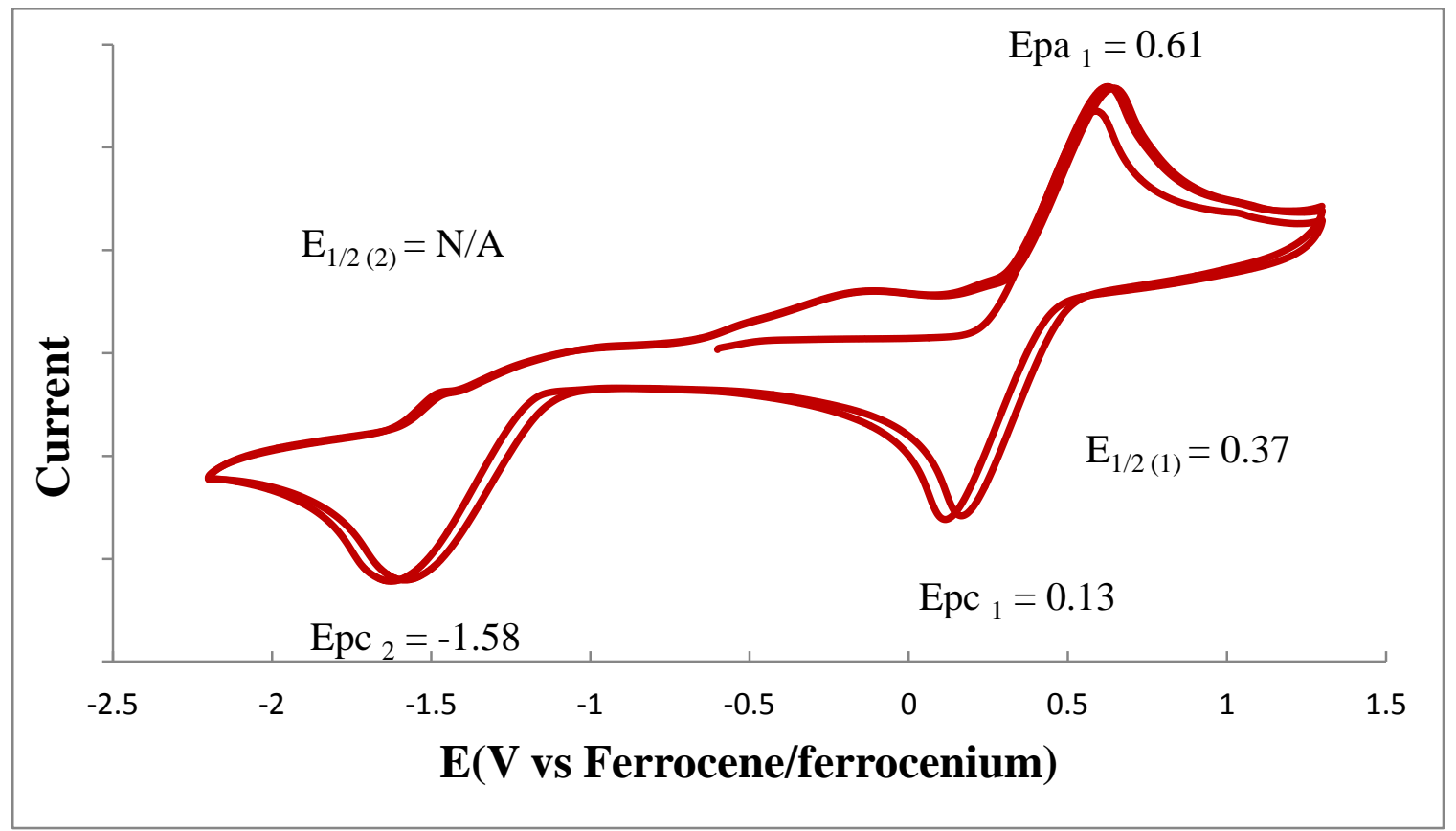


CV Spectrum 3: Bis (1, 5-diisopropyl-6-oxoverdazyl)-3,3'- $\alpha$-quaterthiophene (6)

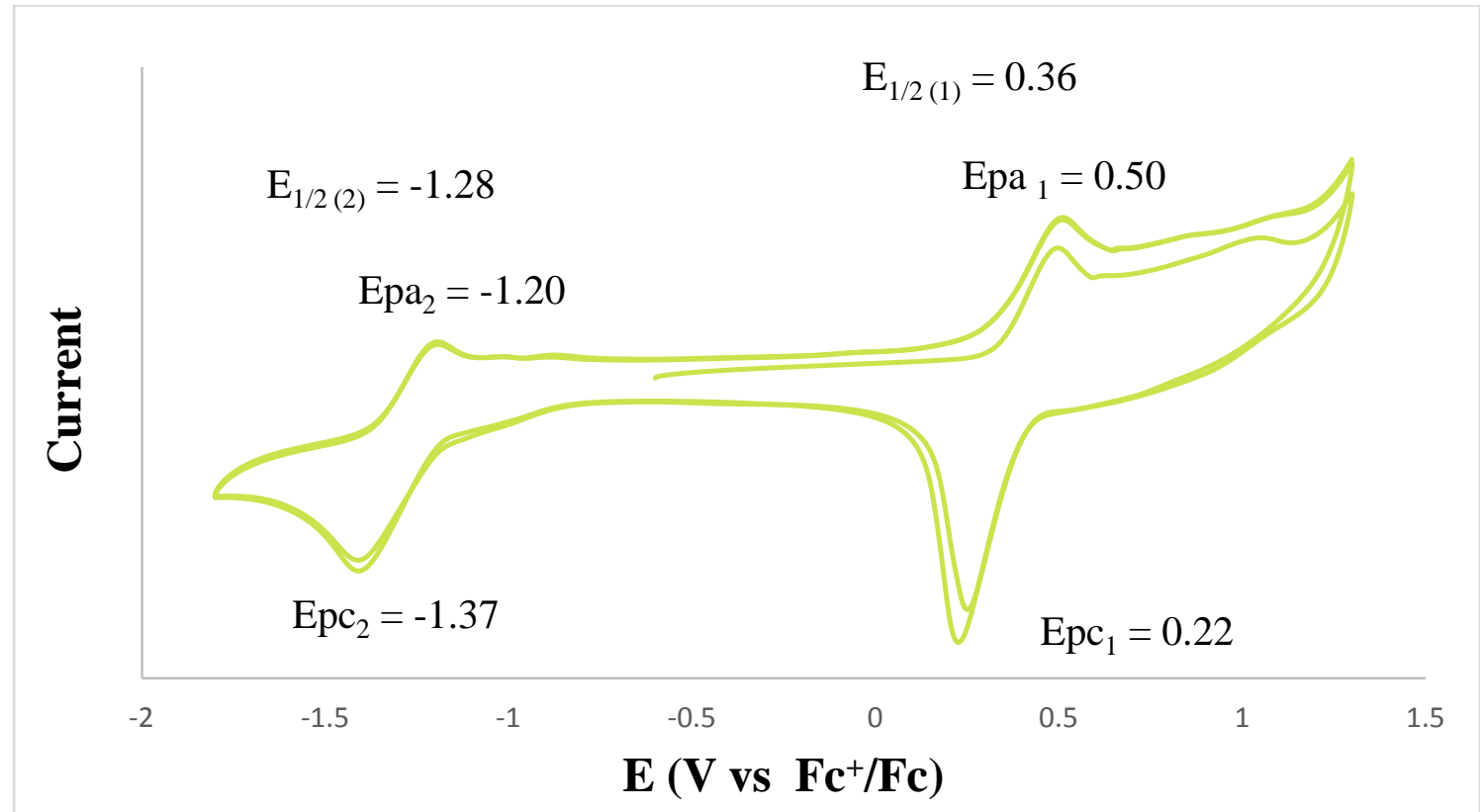

CV Spectrum 4: Bis (1, 5-diisopropyl-6-oxoverdazyl)-3,3'-biphenyl-4,4'-bithiophene (7)

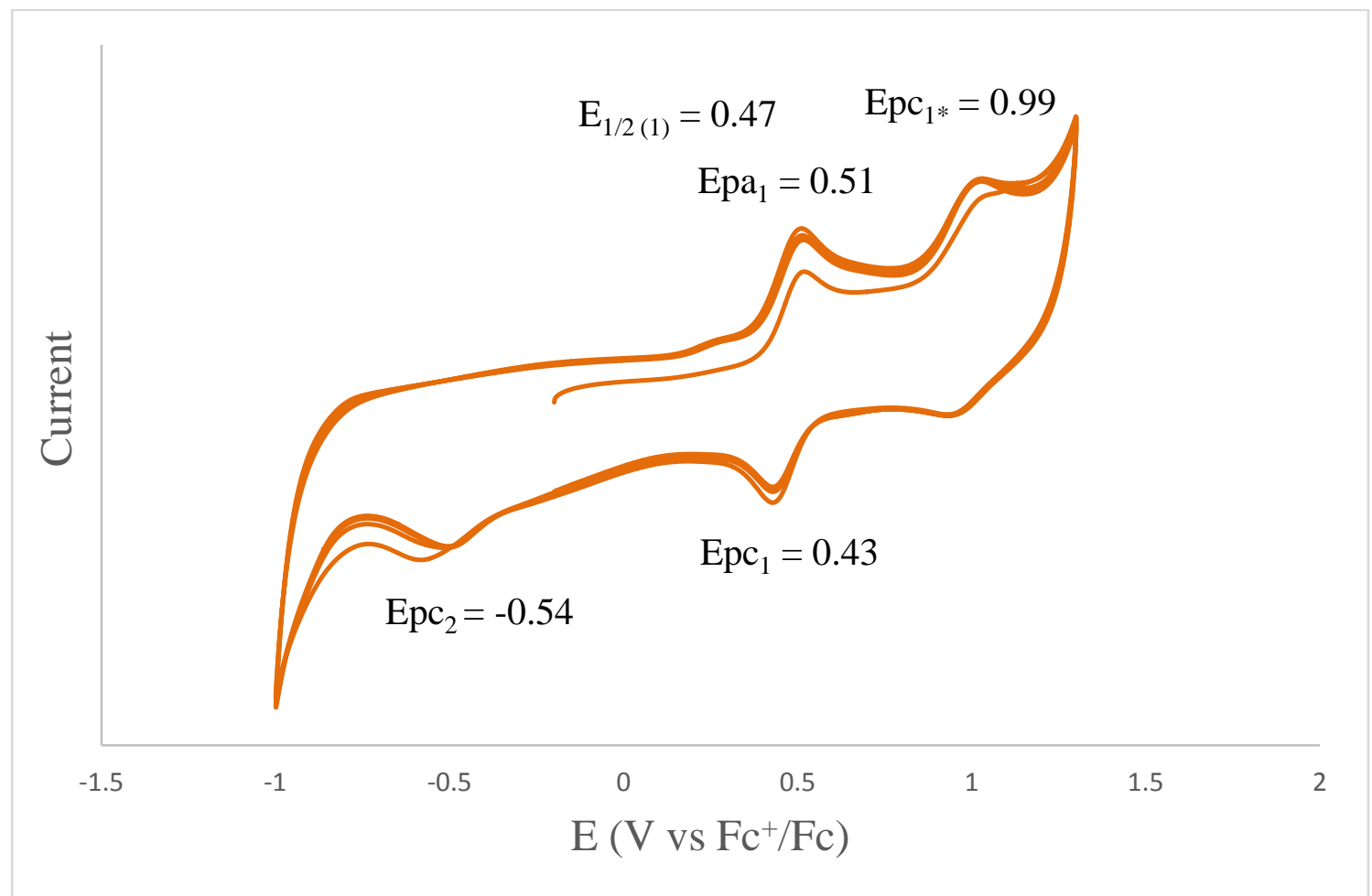




\section{ESR Bisverdazyl Overlays}

ESR Overlay 1: Quaterthiophene Bisverdazyl

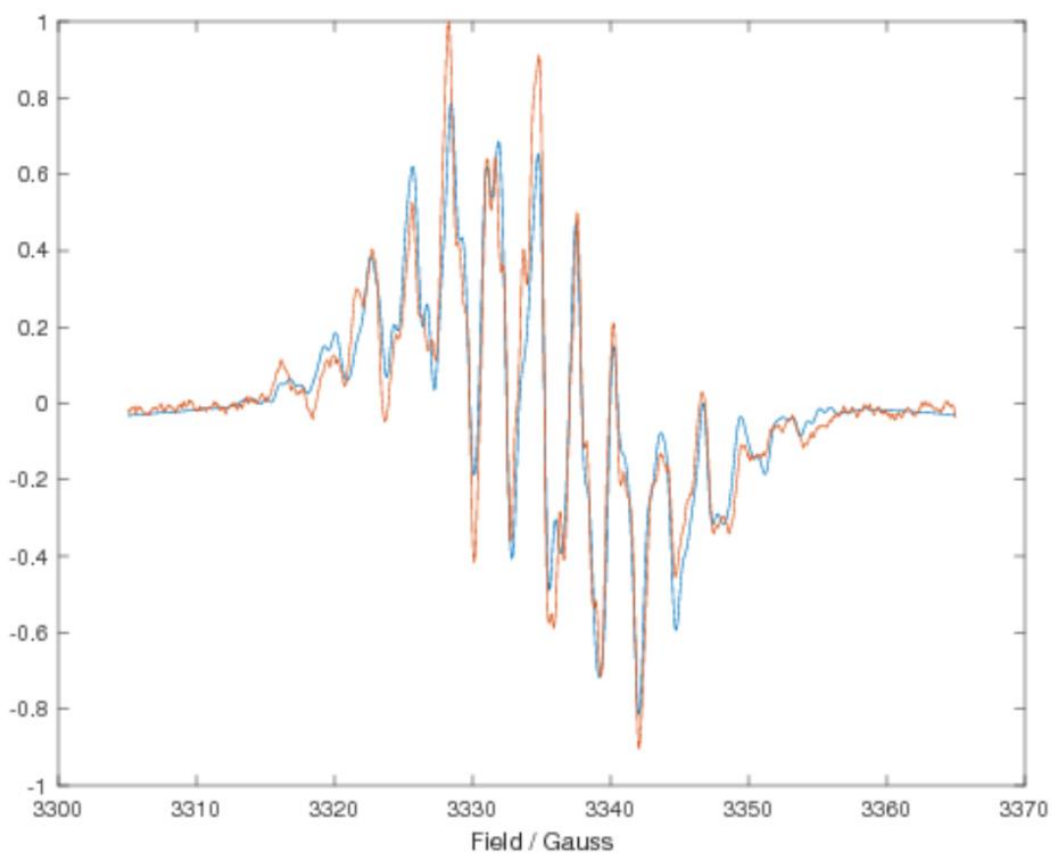

ESR Overlay 2: Biphenyl Bithiophene Bisverdazyl

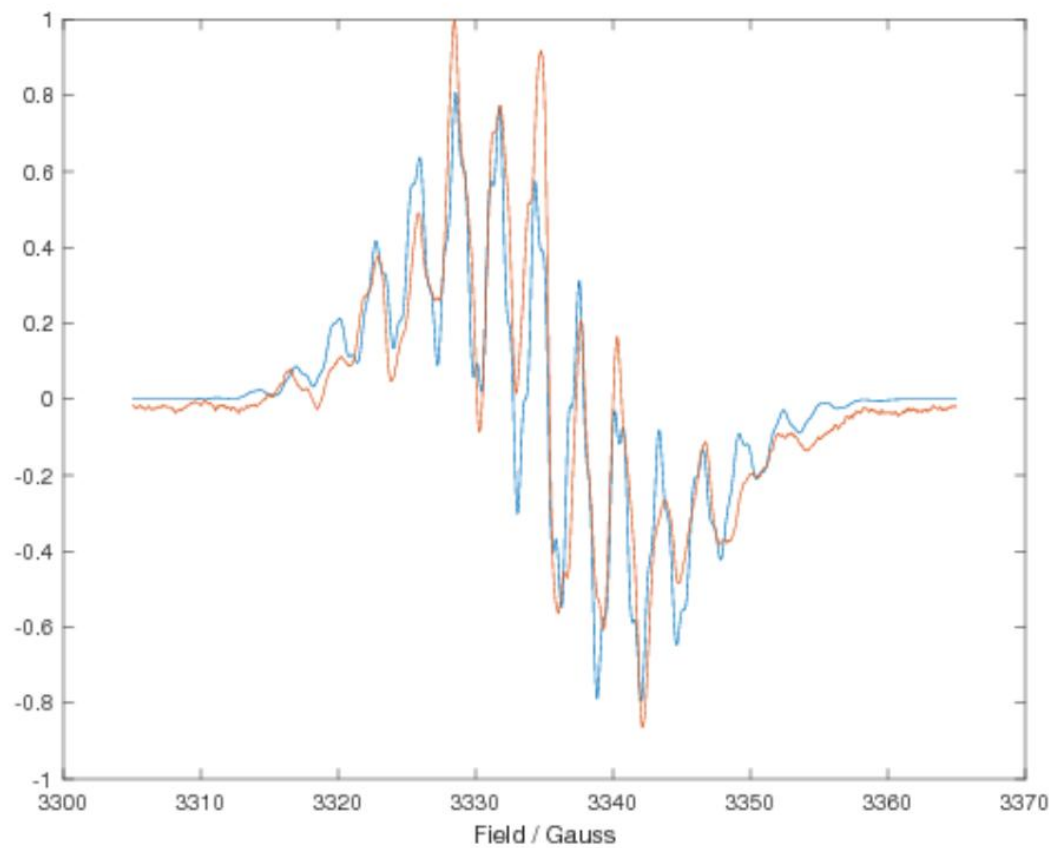




\title{
X-ray Crystallography
}

XRD Data 1: 1,5-Diisopropyl-3-thiophene-6-oxoverdazyl (4)

\section{Crystal Structure Report for verdazyl_thiophene}

\begin{abstract}
A clear red plate-like specimen of $\mathrm{C}_{12} \mathrm{H}_{17} \mathrm{~N}_{4} \mathrm{OS}$, approximate dimensions $0.105 \mathrm{~mm} \times 0.234 \mathrm{~mm} \times 0.518 \mathrm{~mm}$, was used for the X-ray crystallographic analysis. The X-ray intensity data were measured on a Bruker D8 QUEST fixed- $\chi$ goniometer system equipped with a Siemens KFFMO2K-90C sealed tube $(\mathrm{Mo} \mathrm{K \alpha}, \lambda=0.71073 \mathrm{~A}$ ) and a curved graphite monochromator.
\end{abstract}

Table 1: Data collection details for verdazyl_thiophene.

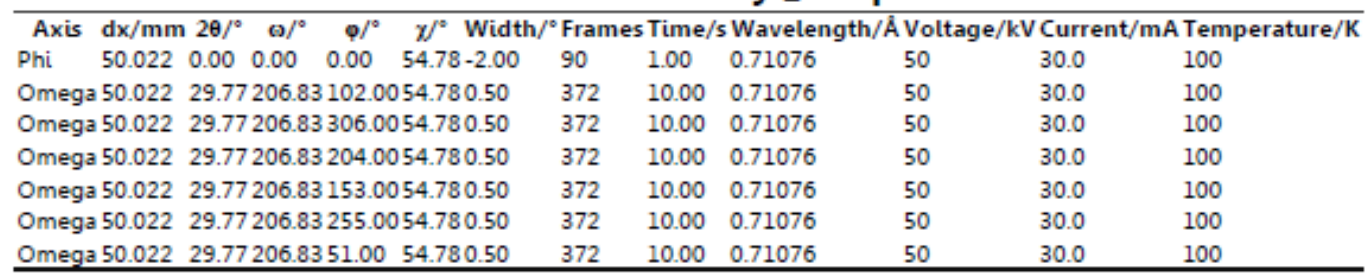

A total of 2322 frames were collected. The total exposure time was 6.22 hours. The frames were integrated with the Bruker SAINT software package using a narrow-frame algorithm. The integration of the data using a monoclinic unit cell yielded a total of 61137 reflections to a maximum $\theta$ angle of $36.33^{\circ}(0.60 \mathrm{~A}$ resolution), of which 6515 were independent (average redundancy 9.384 , completeness $\left.=100.0 \%, R_{\text {int }}=2.62 \%, R_{\text {sig }}=1.37 \%\right)$ and $5590(85.80 \%)$ were greater than $2 \sigma\left(F^{2}\right)$. The final cell constants of $\underline{a}=15.4560(5) \hat{A} \underline{\mathrm{b}}=5.8702(2) \hat{A} \underline{c}=16.1519(6) \AA, \beta=113.6679(11)^{\circ}$, volume $=1342.20(8) \AA^{3}$, are based upon the refinement of the XYZ-centroids of 459 reflections above $20 \sigma(I)$ with $6.150^{\circ}<2 \theta<42.48^{\circ}$. Data were corrected for absorption effects using the Multi-Scan method (SADABS). The ratio of minimum to maximum apparent transmission was 0.950 . The calculated minimum and maximum transmission coefficients (based on crystal size) are 0.7097 and 0.7471 .

The structure was solved and refined using the Bruker SHELXTL Software Package, using the space group P $121 / \mathrm{c} 1$, with Z $=4$ for the formula unit, $\mathrm{C}_{12} \mathrm{H}_{17} \mathrm{~N}_{4} \mathrm{OS}$. The final anisotropic full-matrix least-squares refinement on $\mathrm{F}^{2}$ with 236 variables converged at $R 1=3.07 \%$, for the observed data and $w R 2=9.07 \%$ for all data. The goodness-of-fit was 1.035 . The largest peak in the final difference electron density synthesis was $0.474 \mathrm{e}^{\prime} / \mathbb{A}^{3}$ and the largest hole was $-0.234 \mathrm{e} / \mathbb{A}^{3}$ with an $\mathrm{RMS}$ deviation of $0.044 \mathrm{e}^{-} / \mathbb{A}^{3}$. On the basis of the final model, the calculated density was $1.313 \mathrm{~g} / \mathrm{cm}^{3}$ and $F(000), 564 \mathrm{e}$.

Table 2. Sample and crystal data for verdazyl_thiophene.

\begin{tabular}{|c|c|c|}
\hline Identification code & \multicolumn{2}{|l|}{ verdazylthiophene } \\
\hline Chemical formula & \multicolumn{2}{|l|}{$\mathrm{C}_{12} \mathrm{H}_{17} \mathrm{~N}_{4} \mathrm{OS}$} \\
\hline Formula weight & \multicolumn{2}{|l|}{$265.35 \mathrm{~g} / \mathrm{mol}$} \\
\hline Temperature & \multicolumn{2}{|l|}{$100(2) \mathrm{K}$} \\
\hline Wavelength & \multicolumn{2}{|l|}{$0.71073 \AA$} \\
\hline Crystal size & \multicolumn{2}{|c|}{$0.105 \times 0.234 \times 0.518 \mathrm{~mm}$} \\
\hline Crystal habit & \multicolumn{2}{|l|}{ clear red plate } \\
\hline Crystal system & \multicolumn{2}{|l|}{ monoclinic } \\
\hline Space group & \multicolumn{2}{|l|}{$\mathrm{P} 121 / \mathrm{c} 1$} \\
\hline \multirow[t]{3}{*}{ Unit cell dimensions } & $a=15.4560(5) A$ & $\alpha=90^{\circ}$ \\
\hline & $\mathrm{b}=5.8702(2) \mathrm{A}$ & $\beta=113.6679(11)^{\circ}$ \\
\hline & $c=16.1519(6) \AA$ & $\gamma=90^{\circ}$ \\
\hline Volume & \multicolumn{2}{|l|}{$1342.20(8) A^{3}$} \\
\hline $\mathbf{z}$ & \multicolumn{2}{|l|}{4} \\
\hline Density (calculated) & \multicolumn{2}{|l|}{$1.313 \mathrm{~g} / \mathrm{cm}^{3}$} \\
\hline Absorption coefficient & \multicolumn{2}{|l|}{$0.236 \mathrm{~mm}^{-1}$} \\
\hline$F(000)$ & \multicolumn{2}{|l|}{564} \\
\hline
\end{tabular}


Table 3. Data collection and structure refinement for verdazyl_thiophene.

\begin{tabular}{|c|c|c|}
\hline Diffractometer & \multicolumn{2}{|c|}{ Bruker D8 QUEST fixed- $\chi$ goniometer } \\
\hline Radiation source & \multicolumn{2}{|c|}{$\begin{array}{l}\text { Siemens KFFMO2K-90C sealed tube (Mo Ka, } \lambda= \\
0.71073 \mathrm{~A} \text { ) }\end{array}$} \\
\hline $\begin{array}{l}\text { Theta range for data collection } \\
\text { Index ranges }\end{array}$ & \multicolumn{2}{|c|}{$-25<=h<=25,-9<=k<=9,-26<=l<=26$} \\
\hline $\begin{array}{l}\text { Reflections collected } \\
\text { Independent reflections }\end{array}$ & \multicolumn{2}{|c|}{$6515[R$ (int) $=0.0262]$} \\
\hline $\begin{array}{l}\text { Coverage of independent } \\
\text { reflections }\end{array}$ & \multicolumn{2}{|l|}{$100.0 \%$} \\
\hline Absorption correction & \multicolumn{2}{|l|}{ Multi-Scan } \\
\hline Max. and min. transmission & \multicolumn{2}{|l|}{0.7471 and 0.7097} \\
\hline $\begin{array}{l}\text { Structure solution technique } \\
\text { Structure solution program }\end{array}$ & \multicolumn{2}{|l|}{ direct methods } \\
\hline Refinement method & \multicolumn{2}{|c|}{ Full-matrix least-squares on $\mathrm{F}^{2}$} \\
\hline Refinement program & \multicolumn{2}{|c|}{ SHELXL-2018/3 (Sheldrick, 2018) } \\
\hline Function minimized & \multicolumn{2}{|c|}{$\Sigma w\left(F_{o}^{2}-F_{c}^{2}\right)^{2}$} \\
\hline Data / restraints / parameters & \multicolumn{2}{|l|}{$6515 / 0 / 236$} \\
\hline Goodness-of-fit on $F^{2}$ & \multicolumn{2}{|l|}{1.035} \\
\hline$\Delta / \sigma_{\max }$ & \multicolumn{2}{|l|}{0.001} \\
\hline \multirow[t]{2}{*}{ Final $R$ indices } & 5590 data; $\mid>2 \sigma(l)$ & $R 1=0.0307, w R 2=0.0850$ \\
\hline & all data & $R 1=0.0380, w R 2=0.0907$ \\
\hline Weighting scheme & \multicolumn{2}{|c|}{$\begin{array}{l}\mathrm{w}=1 /\left[\sigma^{2}\left(\mathrm{~F}_{0}^{2}\right)+(0.0466 \mathrm{P})^{2}+0.2479 \mathrm{P}\right] \\
\text { where } \mathrm{P}=\left(\mathrm{Fo}_{\mathrm{o}}^{2}+2 \mathrm{~F}_{\mathrm{c}}^{2}\right) / 3\end{array}$} \\
\hline Largest diff. peak and hole & \multicolumn{2}{|c|}{0.474 and $-0.234 \mathrm{eA}^{-3}$} \\
\hline R.M.S. deviation from mean & \multicolumn{2}{|l|}{$0.044 \mathrm{eA}^{-3}$} \\
\hline
\end{tabular}

Table 4. Atomic coordinates and equivalent isotropic atomic

displacement parameters $\left(\AA^{2}\right)$ for verdazyl_thiophene.

$U(e q)$ is defined as one third of the trace of the orthogonalized $U$, tensor.

\begin{tabular}{lllll}
\hline \multicolumn{1}{c}{ x/a } & \multicolumn{1}{c}{ y/b } & \multicolumn{1}{c}{ z/c } & \multicolumn{1}{c}{ U(eq) } \\
O1 & $0.92549(3)$ & $0.67625(9)$ & $0.54050(3)$ & $0.02072(9)$ \\
N1 & $0.84873(3)$ & $0.65433(8)$ & $0.38649(3)$ & $0.01386(8)$ \\
N2 & $0.77415(3)$ & $0.58555(9)$ & $0.31060(3)$ & $0.01468(8)$ \\
N3 & $0.71542(3)$ & $0.38366(9)$ & $0.40568(3)$ & $0.01499(8)$ \\
N4 & $0.79128(3)$ & $0.46006(9)$ & $0.47875(3)$ & $0.01470(8)$ \\
C1 & $0.86010(4)$ & $0.60266(9)$ & $0.47374(4)$ & $0.01416(9)$ \\
C2 & $0.71247(4)$ & $0.45180(9)$ & $0.32596(3)$ & $0.01336(8)$ \\
C3 & $0.63258(4)$ & $0.36632(10)$ & $0.24659(4)$ & $0.01450(9)$ \\
C5 & $0.50905(5)$ & $0.32099(15)$ & $0.09941(5)$ & $0.02811(14)$ \\
C6 & $0.50371(5)$ & $0.13902(13)$ & $0.14880(5)$ & $0.02334(12)$ \\
C7 & $0.91674(4)$ & $0.80816(10)$ & $0.37134(4)$ & $0.01491(9)$ \\
C8 & $0.87544(5)$ & $0.04697(11)$ & $0.35137(5)$ & $0.02322(12)$ \\
C9 & $0.94263(4)$ & $0.71543(11)$ & $0.29647(4)$ & $0.01961(10)$ \\
C10 & $0.79143(4)$ & $0.39499(10)$ & $0.56731(4)$ & $0.01641(9)$ \\
C11 & $0.70827(5)$ & $0.50702(13)$ & $0.57899(4)$ & $0.02239(12)$ \\
C12 & $0.79013(6)$ & $0.13709(12)$ & $0.57488(5)$ & $0.02457(12)$ \\
S1 & $0.59027(5)$ & $0.51397(12)$ & $0.14710(5)$ & $0.02038(12)$ \\
C4 & $0.5785(2)$ & $0.1706(4)$ & $0.23971(18)$ & $0.0179(4)$ \\
& & & &
\end{tabular}




\begin{tabular}{lllll}
\hline & \multicolumn{1}{c}{$\mathbf{x} / \mathbf{a}$} & \multicolumn{1}{c}{$y / \mathbf{b}$} & \multicolumn{1}{c}{$\mathbf{z} / \mathbf{c}$} & \multicolumn{1}{c}{$\mathbf{U}(\mathrm{eq})$} \\
S1 $^{\prime}$ & $0.58094(5)$ & $0.11682(12)$ & $0.25464(5)$ & $0.01975(13)$ \\
$\mathrm{C4}^{*}$ & $0.5920(2)$ & $0.4590(5)$ & $0.1617(2)$ & $0.0241(5)$ \\
\hline
\end{tabular}

Table 5. Bond lengths $(\AA)$ for verdazyl_thiophene.

\begin{tabular}{|c|c|c|c|}
\hline$\overline{\mathrm{Ol}-\mathrm{Cl}}$ & $1.2230(7)$ & N1-N2 & $1.3637(6)$ \\
\hline $\mathrm{N} 1-\mathrm{Cl}$ & $1.3821(7)$ & $\mathrm{N} 1-\mathrm{Cl}$ & $1.4788(7)$ \\
\hline $\mathrm{N} 2-\mathrm{C} 2$ & $1.3330(7)$ & $\mathrm{N} 3-\mathrm{C} 2$ & $1.3315(7)$ \\
\hline N3-N4 & $1.3630(7)$ & $\mathrm{N} 4-\mathrm{Cl}$ & $1.3812(7)$ \\
\hline $\mathrm{N} 4-\mathrm{C} 10$ & $1.4796(7)$ & $\mathrm{C} 2-\mathrm{C}_{3}$ & $1.4660(7)$ \\
\hline $\mathrm{C} 3-\mathrm{CH}^{\prime}$ & $1.371(3)$ & $\mathrm{C} 3-\mathrm{C} 4$ & $1.399(3)$ \\
\hline C3-51' & $1.6978(8)$ & $C 3-51$ & $1.7081(8)$ \\
\hline$C 5-C 6$ & $1.3554(10)$ & $\mathrm{C} 5-\mathrm{CH}^{\circ}$ & $1.509(3)$ \\
\hline$C 5-S 1$ & $1.6359(10)$ & $\mathrm{C} 5-\mathrm{H} 5$ & $0.947(12)$ \\
\hline$C 6-C 4$ & $1.471(3)$ & C6-51 & $1.6511(10)$ \\
\hline $\mathrm{C} 6-\mathrm{H} 6$ & $0.957(11)$ & $\mathrm{C} 7-\mathrm{Cg}$ & $1.5190(9)$ \\
\hline $\mathrm{C} 7-\mathrm{CB}$ & $1.5203(9)$ & $\mathrm{C} 7-\mathrm{H} 7$ & $0.959(10)$ \\
\hline C8-H8A & $0.975(12)$ & $\mathrm{CB}-\mathrm{H} 8 \mathrm{~B}$ & $0.976(12)$ \\
\hline $\mathrm{CB}-\mathrm{H} 8 \mathrm{C}$ & $0.984(12)$ & $\mathrm{Cg}-\mathrm{H} 9 \mathrm{~A}$ & $0.975(11)$ \\
\hline С9-H9B & $0.967(12)$ & C9-H9C & $0.974(11)$ \\
\hline $\mathrm{C} 10-\mathrm{Cl}_{2}$ & $1.5196(9)$ & $\mathrm{C}_{10}-\mathrm{C}_{11}$ & $1.5215(9)$ \\
\hline $\mathrm{ClO}-\mathrm{H} 10$ & $0.964(10)$ & C11-H11A & $0.966(12)$ \\
\hline C11-H11B & $0.997(12)$ & C11-H11C & $0.955(12)$ \\
\hline $\mathrm{C} 12-\mathrm{H} 12 \mathrm{~A}$ & $0.997(12)$ & $\mathrm{C} 12-\mathrm{H} 12 \mathrm{~B}$ & $1.009(12)$ \\
\hline $\mathrm{C} 12-\mathrm{H} 12 \mathrm{C}$ & $0.970(13)$ & $\mathrm{C} 4-\mathrm{H} 4$ & $1.13(2)$ \\
\hline $\mathrm{C} 4^{\circ}-\mathrm{H} 4^{\prime}$ & $1.10(3)$ & & \\
\hline
\end{tabular}

Table 6 . Bond angles $\left({ }^{\circ}\right)$ for verdazyl_thiophene.

\begin{tabular}{|c|c|c|c|}
\hline $\mathrm{N} 2-\mathrm{N} 1-\mathrm{Cl}$ & $124.55(5)$ & $\mathrm{N} 2-\mathrm{N} 1-\mathrm{C} 7$ & $115.63(4)$ \\
\hline C1-N1-C7 & $119.66(4)$ & $\mathrm{C} 2-\mathrm{N} 2-\mathrm{N} 1$ & $114.63(5)$ \\
\hline $\mathrm{C} 2-\mathrm{N} 3-\mathrm{N} 4$ & $114.97(5)$ & N3-N4-C1 & $124.31(5)$ \\
\hline N3-N4-C10 & $114.78(5)$ & $\mathrm{Cl}-\mathrm{N} 4-\mathrm{C} 10$ & $120.71(5)$ \\
\hline O1-C1-N4 & $123.07(5)$ & O1-Cl-N1 & $122.91(5)$ \\
\hline $\mathrm{N} 4-\mathrm{Cl}-\mathrm{N} 1$ & $114.02(5)$ & $\mathrm{N} 3-\mathrm{C} 2-\mathrm{N} 2$ & $127.41(5)$ \\
\hline $\mathrm{N} 3-\mathrm{C}_{2}-\mathrm{C}_{3}$ & $115.64(5)$ & $\mathrm{N} 2-\mathrm{C} 2-\mathrm{C} 3$ & $116.94(5)$ \\
\hline$C 4^{\prime}-\mathrm{C} 3-\mathrm{C} 2$ & $128.70(13)$ & $\mathrm{C} 4-\mathrm{C} 3-\mathrm{C} 2$ & $128.13(11)$ \\
\hline$C 4^{\prime}-C 3-51^{\prime}$ & $112.26(13)$ & $\mathrm{C} 2-\mathrm{C}_{3}-\mathrm{SI}^{\prime}$ & $119.02(5)$ \\
\hline C4-C3-S1 & $110.55(11)$ & $C 2-C 3-S 1$ & $121.27(5)$ \\
\hline $\mathrm{C} 6-\mathrm{C} 5-\mathrm{C}^{\prime}$ & $105.50(11)$ & C6-C5-S1 & $119.03(6)$ \\
\hline $\mathrm{C} 6-\mathrm{C} 5-\mathrm{H} 5$ & $126.3(7)$ & $\mathrm{C} 4^{\prime}-\mathrm{C} 5-\mathrm{H} 5$ & $128.2(7)$ \\
\hline $\mathrm{S} 1-\mathrm{C} 5-\mathrm{H} 5$ & $114.7(7)$ & $C 5-C 6-C 4$ & $106.21(10)$ \\
\hline C5-C6-S1' & $118.82(6)$ & $\mathrm{C} 5-\mathrm{C} 6-\mathrm{H} 6$ & $125.6(7)$ \\
\hline $\mathrm{C} 4-\mathrm{C} 6-\mathrm{H} 6$ & $128.2(7)$ & $\mathrm{S} 1^{\prime}-\mathrm{C} 6-\mathrm{H} 6$ & $115.6(7)$ \\
\hline $\mathrm{N} 1-\mathrm{C} 7-\mathrm{Cg}$ & $110.17(5)$ & $\mathrm{N} 1-\mathrm{C} 7-\mathrm{C} 8$ & $109.41(5)$ \\
\hline $\mathrm{CP}-\mathrm{C} 7-\mathrm{CB}$ & $112.57(5)$ & $\mathrm{N} 1-\mathrm{C} 7-\mathrm{H} 7$ & $103.7(6)$ \\
\hline $\mathrm{C}-\mathrm{C} 7-\mathrm{H} 7$ & $110.5(6)$ & $\mathrm{C} 8-\mathrm{C} 7-\mathrm{H} 7$ & $110.1(6)$ \\
\hline C7-C8-H8A & $109.6(7)$ & $\mathrm{C} 7-\mathrm{C} 8-\mathrm{H} 8 \mathrm{~B}$ & $110.4(7)$ \\
\hline $\mathrm{H} 8 \mathrm{~A}-\mathrm{C} 8-\mathrm{H} 8 \mathrm{~B}$ & $108.4(10)$ & $\mathrm{C} 7-\mathrm{C} 8-\mathrm{H} 8 \mathrm{C}$ & $109.6(7)$ \\
\hline $\mathrm{H} 8 \mathrm{~A}-\mathrm{C} 8-\mathrm{H} 8 \mathrm{C}$ & $109.9(10)$ & $\mathrm{H} 8 \mathrm{~B}-\mathrm{C} 8-\mathrm{H} 8 \mathrm{C}$ & $109.0(10)$ \\
\hline $\mathrm{C} 7-\mathrm{Cg}-\mathrm{H} 9 \mathrm{~A}$ & $112.4(7)$ & C7-C9-H9B & $111.1(7)$ \\
\hline $\mathrm{H} 9 \mathrm{~A}-\mathrm{C} 9-\mathrm{H} 9 \mathrm{~B}$ & $107.1(9)$ & $\mathrm{C} 7-\mathrm{Cg}-\mathrm{H} 9 \mathrm{C}$ & $108.9(7)$ \\
\hline $\mathrm{H} 9 \mathrm{~A}-\mathrm{C}-\mathrm{H} 9 \mathrm{C}$ & $109.1(9)$ & $\mathrm{H} 9 \mathrm{~B}-\mathrm{Cg}-\mathrm{H} 9 \mathrm{C}$ & $108.2(9)$ \\
\hline
\end{tabular}




\begin{tabular}{llll}
$\mathrm{N} 4-\mathrm{C} 10-\mathrm{C} 12$ & $109.88(5)$ & $\mathrm{N} 4-\mathrm{C} 10-\mathrm{C} 11$ & $109.56(5)$ \\
$\mathrm{C} 12-\mathrm{C} 10-\mathrm{C} 11$ & $112.43(6)$ & $\mathrm{N} 4-\mathrm{C} 10-\mathrm{H} 10$ & $103.8(6)$ \\
$\mathrm{C} 12-\mathrm{C} 10-\mathrm{H} 10$ & $110.8(6)$ & $\mathrm{C} 11-\mathrm{C} 10-\mathrm{H} 10$ & $110.0(6)$ \\
$\mathrm{C} 10-\mathrm{C} 11-\mathrm{H} 11 \mathrm{~A}$ & $109.8(7)$ & $\mathrm{C} 10-\mathrm{C} 11-\mathrm{H} 11 \mathrm{~B}$ & $110.3(7)$ \\
$\mathrm{H} 11 \mathrm{~A}-\mathrm{C} 11-\mathrm{H} 11 \mathrm{~B}$ & $109.4(10)$ & $\mathrm{C} 10-\mathrm{C} 11-\mathrm{H} 11 \mathrm{C}$ & $111.5(7)$ \\
$\mathrm{H} 11 \mathrm{~A}-\mathrm{C} 11-\mathrm{H} 11 \mathrm{C}$ & $110.7(10)$ & $\mathrm{H} 11 \mathrm{~B}-\mathrm{C} 11-\mathrm{H} 11 \mathrm{C}$ & $105.1(10)$ \\
$\mathrm{C} 10-\mathrm{C} 12-\mathrm{H} 12 \mathrm{~A}$ & $110.1(7)$ & $\mathrm{C} 10-\mathrm{C} 12-\mathrm{H} 12 \mathrm{~B}$ & $109.2(7)$ \\
$\mathrm{H} 12 \mathrm{~A}-\mathrm{C} 12-\mathrm{H} 12 \mathrm{~B}$ & $109.9(10)$ & $\mathrm{C} 10-\mathrm{C} 12-\mathrm{H} 12 \mathrm{C}$ & $111.2(7)$ \\
$\mathrm{H} 12 \mathrm{~A}-\mathrm{C} 12-\mathrm{H} 12 \mathrm{C}$ & $109.0(10)$ & $\mathrm{H} 12 \mathrm{~B}-\mathrm{C} 12-\mathrm{H} 12 \mathrm{C}$ & $107.5(10)$ \\
$\mathrm{C} 5-\mathrm{S} 1-\mathrm{C} 3$ & $91.33(4)$ & $\mathrm{C} 3-\mathrm{C} 4-\mathrm{C} 6$ & $112.82(15)$ \\
$\mathrm{C} 3-\mathrm{C} 4-\mathrm{H} 4$ & $120.2(11)$ & $\mathrm{C} 6-\mathrm{C} 4-\mathrm{H} 4$ & $126.9(11)$ \\
$\mathrm{C} 6-\mathrm{S} 1^{\prime}-\mathrm{C} 3$ & $91.11(4)$ & $\mathrm{C} 3-\mathrm{C} 4^{\circ}-\mathrm{C} 5$ & $112.24(18)$ \\
$\mathrm{C} 3-\mathrm{C} 4^{\prime}-\mathrm{H} 4$ & $123.7(12)$ & $\mathrm{C} 5-\mathrm{C} 4^{\circ}-\mathrm{H} 4$ & $123.9(12)$ \\
\hline
\end{tabular}

Table 7. Torsion angles $\left({ }^{\circ}\right)$ for verdazyl_thiophene.

\begin{tabular}{|c|c|c|c|}
\hline $\mathrm{C} 1-\mathrm{N} 1-\mathrm{N} 2-\mathrm{C} 2$ & $-2.94(8)$ & $\mathrm{C} 7-\mathrm{N} 1-\mathrm{N} 2-\mathrm{C} 2$ & $-178.35(5)$ \\
\hline $\mathrm{C} 2-\mathrm{N} 3-\mathrm{N} 4-\mathrm{Cl}$ & $1.75(8)$ & $\mathrm{C} 2-\mathrm{N} 3-\mathrm{N} 4-\mathrm{C} 10$ & $176.63(5)$ \\
\hline $\mathrm{N} 3-\mathrm{N} 4-\mathrm{Cl}-\mathrm{O} 1$ & $176.96(6)$ & $\mathrm{Cl}-\mathrm{N} 4-\mathrm{Cl}-\mathrm{O} 1$ & $2.37(9)$ \\
\hline N3-N4-Cl-N1 & $-3.25(8)$ & $\mathrm{Cl}-\mathrm{N} 4-\mathrm{Cl}-\mathrm{N} 1$ & $-177.84(5)$ \\
\hline $\mathrm{N} 2-\mathrm{N} 1-\mathrm{Cl}-\mathrm{O} 1$ & $-176.33(6)$ & $\mathrm{C} 7-\mathrm{N} 1-\mathrm{Cl}-\mathrm{O} 1$ & $-1.09(8)$ \\
\hline $\mathrm{N} 2-\mathrm{N} 1-\mathrm{Cl}-\mathrm{N} 4$ & $3.89(8)$ & $\mathrm{C} 7-\mathrm{N} 1-\mathrm{Cl}-\mathrm{N} 4$ & $179.13(5)$ \\
\hline $\mathrm{N} 4-\mathrm{N} 3-\mathrm{C} 2-\mathrm{N} 2$ & $-0.63(9)$ & $\mathrm{N} 4-\mathrm{N} 3-\mathrm{C}_{2}-\mathrm{C}_{3}$ & $178.50(5)$ \\
\hline $\mathrm{N} 1-\mathrm{N} 2-\mathrm{C} 2-\mathrm{N} 3$ & $1.18(9)$ & $\mathrm{N} 1-\mathrm{N} 2-\mathrm{C} 2-\mathrm{C} 3$ & $-177.94(5)$ \\
\hline $\mathrm{N} 3-\mathrm{C} 2-\mathrm{C}^{-}-\mathrm{Cl}^{\prime}$ & $155.5(2)$ & $\mathrm{N} 2-\mathrm{C} 2-\mathrm{C} 3-\mathrm{Cl}^{\prime}$ & $-25.2(2)$ \\
\hline $\mathrm{N} 3-\mathrm{C} 2-\mathrm{C} 3-\mathrm{C} 4$ & $-23.63(17)$ & $\mathrm{N} 2-\mathrm{C} 2-\mathrm{C} 3-\mathrm{C} 4$ & $155.60(16)$ \\
\hline N3-C2-C3-SI' & $-26.01(8)$ & $\mathrm{N} 2-\mathrm{C} 2-\mathrm{C} 3-\mathrm{S} 1^{\prime}$ & $153.22(5)$ \\
\hline N3-C2-C3-S1 & $153.43(5)$ & $\mathrm{N} 2-\mathrm{C} 2-\mathrm{C} 3-\mathrm{S} 1$ & $-27.34(8)$ \\
\hline $\mathrm{S} 1-\mathrm{C} 5-\mathrm{C} 6-\mathrm{C} 4$ & $0.66(15)$ & $\mathrm{C} 4^{\prime}-\mathrm{C} 5-\mathrm{C} 6-\mathrm{S} 1^{\prime}$ & $0.54(17)$ \\
\hline $\mathrm{N} 2-\mathrm{N} 1-\mathrm{C} 7-\mathrm{Cg}$ & $-47.38(6)$ & $\mathrm{Cl}-\mathrm{N} 1-\mathrm{C} 7-\mathrm{Cg}$ & $136.97(5)$ \\
\hline $\mathrm{N} 2-\mathrm{N} 1-\mathrm{C} 7-\mathrm{C} 8$ & $76.89(6)$ & $\mathrm{Cl}-\mathrm{N} 1-\mathrm{C} 7-\mathrm{C} 8$ & $-98.77(6)$ \\
\hline $\mathrm{N} 3-\mathrm{N} 4-\mathrm{Cl} 10-\mathrm{C} 12$ & $59.36(7)$ & $\mathrm{C} 1-\mathrm{N} 4-\mathrm{C} 10-\mathrm{C} 12$ & $-125.56(6)$ \\
\hline N3-N4-C10-C11 & $-64.63(7)$ & $\mathrm{C} 1-\mathrm{N} 4-\mathrm{C} 10-\mathrm{C} 11$ & $110.45(6)$ \\
\hline C6-C5-S1-C3 & $0.75(8)$ & C4-C3-S1-C5 & $-1.99(14)$ \\
\hline C2-C3-S1-C5 & $-179.52(5)$ & $\mathrm{C} 2-\mathrm{C} 3-\mathrm{C} 4-\mathrm{C} 6$ & $-179.93(10)$ \\
\hline $\mathrm{S1}-\mathrm{C} 3-\mathrm{C} 4-\mathrm{C} 6$ & $2.8(2)$ & $\mathrm{C} 5-\mathrm{C} 6-\mathrm{C} 4-\mathrm{C} 3$ & $-2.2(2)$ \\
\hline $\mathrm{C} 5-\mathrm{C} 6-51^{\prime}-\mathrm{C3}$ & $0.94(8)$ & $C 4^{\prime}-\mathrm{C} 3-51^{\prime}-\mathrm{C} 6$ & $-2.28(17)$ \\
\hline $\mathrm{C} 2-\mathrm{C} 3-51^{\prime}-\mathrm{C} 6$ & $179.03(5)$ & $\mathrm{C} 2-\mathrm{C} 3-\mathrm{C} 4^{\prime}-\mathrm{C} 5$ & $-178.47(11)$ \\
\hline$S 1^{\prime}-C 3-C 4^{\prime}-C 5$ & $3.0(3)$ & $\mathrm{C} 6-\mathrm{C} 5-\mathrm{C} 4^{\prime}-\mathrm{C} 3$ & $-2.2(3)$ \\
\hline
\end{tabular}

Table 8. Anisotropic atomic displacement parameters $\left(\AA^{2}\right)$ for verdazyl_thiophene.

The anisotropic atomic displacement factor exponent takes the form: $-2 \pi^{2}\left[\mathrm{~h}^{2} \mathrm{a}^{2} \mathrm{U}_{11}+\ldots+2 \mathrm{~h} \mathrm{k} \mathrm{a}^{*} \mathrm{~b}^{*}\right.$ $\left.\mathrm{U}_{12}\right]$

\begin{tabular}{lllllcc}
\hline & \multicolumn{1}{c}{$\mathbf{U}_{11}$} & \multicolumn{1}{c}{$\mathbf{U}_{22}$} & $\mathbf{U}_{33}$ & \multicolumn{1}{c}{$\mathbf{U}_{23}$} & $\mathbf{U}_{13}$ & $\mathbf{U}_{12}$ \\
$\mathrm{O} 1$ & $0.01827(19)$ & $0.0260(2)$ & $0.01340(17)$ & $-0.00181(15)$ & $0.00161(14)$ & $-0.00571(16)$ \\
$\mathrm{N} 1$ & $0.01190(17)$ & $0.01650(19)$ & $0.01194(17)$ & $-0.00055(14)$ & $0.00350(14)$ & $-0.00231(14)$ \\
$\mathrm{N} 2$ & $0.01231(18)$ & $0.0180(2)$ & $0.01219(17)$ & $-0.00089(14)$ & $0.00335(14)$ & $-0.00282(14)$ \\
$\mathrm{N} 3$ & $0.01362(18)$ & $0.0189(2)$ & $0.01159(17)$ & $-0.00089(15)$ & $0.00412(14)$ & $-0.00192(15)$ \\
$\mathrm{N} 4$ & $0.01392(18)$ & $0.0189(2)$ & $0.01073(17)$ & $-0.00039(14)$ & $0.00436(14)$ & $-0.00147(15)$ \\
$\mathrm{C} 1$ & $0.0134(2)$ & $0.0154(2)$ & $0.01268(19)$ & $-0.00042(16)$ & $0.00413(16)$ & $0.00035(16)$ \\
$\mathrm{C} 2$ & $0.01181(19)$ & $0.0160(2)$ & $0.01189(19)$ & $-0.00088(15)$ & $0.00435(15)$ & $-0.00095(15)$ \\
$\mathrm{C} 3$ & $0.01256(19)$ & $0.0188(2)$ & $0.01192(19)$ & $-0.00102(16)$ & $0.00473(16)$ & $-0.00337(16)$ \\
$\mathrm{C} 5$ & $0.0271(3)$ & $0.0382(4)$ & $0.0178(3)$ & $0.0018(2)$ & $0.0078(2)$ & $-0.0089(3)$
\end{tabular}




\begin{tabular}{lllllll}
\hline & \multicolumn{1}{c}{$\mathbf{U}_{11}$} & \multicolumn{1}{c}{$\mathbf{U}_{22}$} & \multicolumn{1}{c}{$\mathbf{U}_{33}$} & \multicolumn{1}{c}{$\mathbf{U}_{23}$} & \multicolumn{1}{c}{$\mathbf{U}_{13}$} & \multicolumn{1}{c}{$\mathbf{U}_{12}$} \\
$C 6$ & $0.0182(2)$ & $0.0313(3)$ & $0.0202(3)$ & $-0.0027(2)$ & $0.0074(2)$ & $-0.0099(2)$ \\
C7 & $0.01198(19)$ & $0.0156(2)$ & $0.0156(2)$ & $0.00043(16)$ & $0.00394(16)$ & $-0.00225(16)$ \\
$C 8$ & $0.0230(3)$ & $0.0139(2)$ & $0.0297(3)$ & $-0.0019(2)$ & $0.0074(2)$ & $-0.00096(19)$ \\
$C 9$ & $0.0188(2)$ & $0.0214(2)$ & $0.0217(2)$ & $0.0008(2)$ & $0.0113(2)$ & $-0.00145(19)$ \\
$C 10$ & $0.0171(2)$ & $0.0203(2)$ & $0.01141(19)$ & $0.00170(17)$ & $0.00521(17)$ & $0.00089(17)$ \\
$C 11$ & $0.0245(3)$ & $0.0278(3)$ & $0.0185(2)$ & $0.0034(2)$ & $0.0124(2)$ & $0.0058(2)$ \\
$C 12$ & $0.0314(3)$ & $0.0204(3)$ & $0.0211(3)$ & $0.0059(2)$ & $0.0097(2)$ & $0.0040(2)$ \\
S1 & $0.01855(16)$ & $0.0257(3)$ & $0.0133(2)$ & $0.00248(15)$ & $0.00268(14)$ & $-0.00816(18)$ \\
$C 4$ & $0.0175(6)$ & $0.0222(10)$ & $0.0143(8)$ & $-0.0006(6)$ & $0.0065(6)$ & $-0.0045(7)$ \\
S1 & $0.01812(17)$ & $0.0231(3)$ & $0.0163(3)$ & $-0.00074(17)$ & $0.00511(17)$ & $-0.00874(19)$ \\
$C 4$ & $0.0205(7)$ & $0.0309(13)$ & $0.0189(10)$ & $-0.0001(7)$ & $0.0059(7)$ & $-0.0103(9)$ \\
\hline
\end{tabular}

Table 9. Hydrogen atomic coordinates and isotropic atomic displacement parameters $\left(\AA^{2}\right)$ for verdazyl_thiophene.

\begin{tabular}{lllll}
\hline & \multicolumn{1}{c}{$\mathbf{x} / \mathbf{a}$} & \multicolumn{1}{c}{$\mathbf{y} / \mathbf{b}$} & \multicolumn{1}{c}{$\mathbf{z} / \mathbf{c}$} & \multicolumn{1}{c}{$\mathbf{U}(\mathrm{eq})$} \\
H5 & $0.4699(8)$ & $0.349(2)$ & $0.0380(8)$ & 0.034 \\
H6 & $0.4592(8)$ & $0.0169(18)$ & $0.1273(8)$ & 0.028 \\
H7 & $0.9706(7)$ & $0.8061(17)$ & $0.4280(7)$ & 0.018 \\
H8A & $0.9207(8)$ & $1.150(2)$ & $0.3427(8)$ & 0.035 \\
H8B & $0.8620(8)$ & $1.103(2)$ & $0.4018(8)$ & 0.035 \\
H8C & $0.8164(8)$ & $1.044(2)$ & $0.2965(8)$ & 0.035 \\
H9A & $0.8891(8)$ & $0.7152(19)$ & $0.2379(8)$ & 0.029 \\
H9B & $0.9652(8)$ & $0.560(2)$ & $0.3089(8)$ & 0.029 \\
H9C & $0.9930(8)$ & $0.8087(19)$ & $0.2925(8)$ & 0.029 \\
H10 & $0.8499(7)$ & $0.4567(17)$ & $0.6104(7)$ & 0.02 \\
H11A & $0.7118(8)$ & $0.670(2)$ & $0.5739(8)$ & 0.034 \\
H11B & $0.7087(9)$ & $0.468(2)$ & $0.6392(8)$ & 0.034 \\
H11C & $0.6494(9)$ & $0.451(2)$ & $0.5361(8)$ & 0.034 \\
H12A & $0.7321(9)$ & $0.074(2)$ & $0.5261(8)$ & 0.037 \\
H12B & $0.7916(8)$ & $0.095(2)$ & $0.6360(8)$ & 0.037 \\
H12C & $0.8453(9)$ & $0.069(2)$ & $0.5702(8)$ & 0.037 \\
H4 & $0.5964(15)$ & $0.052(4)$ & $0.2993(16)$ & 0.022 \\
H4 & $0.6151(17)$ & $0.621(4)$ & $0.1423(16)$ & 0.029 \\
\hline
\end{tabular}


XRD Data 2: 1,5-Diisopropyl-3-thiophene-5-boronic acid pinacol ester-6-oxoverdazyl

(5)

\section{Crystal Structure Report for vdthB}

A clear red block-like specimen of $\mathrm{C}_{18} \mathrm{H}_{28} \mathrm{BN}_{4} \mathrm{O}_{3} \mathrm{~S}$, approximate dimensions $0.163 \mathrm{~mm} \times 0.288 \mathrm{~mm} \times 0.381 \mathrm{~mm}$, was used for the X-ray crystallographic analysis. The X-ray intensity data were measured on a Bruker D8 QUEST fixed- $\chi$ goniometer system equipped with a Siemens KFFMO2K-90C sealed tube (Mo $K \alpha, \lambda=0.71073 \mathrm{~A})$ and a curved graphite monochromator.

\section{Table 1: Data collection details for vdthB.}

\begin{tabular}{|c|c|c|c|c|c|c|c|c|c|c|}
\hline Axis & $\mathrm{dx} / \mathrm{mm}$ & $2 \theta /^{\circ}$ & $\varphi / /^{\circ}$ & $\gamma^{\circ}$ Wid & $/{ }^{\circ}$ Frames & s Time & /s Wavelen & Vol & /Curre & A Tempe \\
\hline Phi & 35.026 & $19.59-147.66$ & 50.00 & 54.780 .50 & 720 & 5.00 & 0.71076 & 50 & 30.0 & 100 \\
\hline Omega & 35.026 & $71.73-95.53$ & -12.8 & 054.780 .50 & 309 & 5.00 & 0.71076 & 50 & 30.0 & 100 \\
\hline
\end{tabular}

A total of 1029 frames were collected. The total exposure time was 1.43 hours. The frames were integrated with the Bruker SAINT software package using a narrow-frame algorithm. The integration of the data using a tetragonal unit cell yielded a total of 87798 reflections to a maximum $\theta$ angle of $33.14^{\circ}$ ( $0.65 \mathrm{~A}$ resolution), of which 8069 were independent (average redundancy 10.881 , completeness $=100.0 \%$, Rint $=4.78 \%$, $\left.R_{\text {sig }}=2.23 \%\right)$ and $6504(80.60 \%)$ were greater than $2 \sigma\left(F^{2}\right) . T$ The final cell constants of $\underline{a}=27.5302(14) \mathcal{A}, \underline{b}=27.5302(14) A, \underline{c}=11.1577(7) A$, volume $=8456.6(10) A^{3}$, are based upon the refinement of the $X Y Z$-centroids of 9971 reflections above $20 \sigma(I)$ with $4.928^{\circ}<2 \theta<66.15^{\circ}$. Data were corrected for absorption effects using the Multi-Scan method (SADABS). The ratio of minimum to maximum apparent transmission was 0.939. The calculated minimum and maximum transmission coefficients (based on crystal size) are 0.7013 and 0.7465 .

The structure was solved and refined using the Bruker SHELXTL Software Package, using the space group | 41/a, with Z = 16 for the formula unit, $\mathrm{C}_{18 \mathrm{H}_{28} \mathrm{BN}} \mathrm{O}_{3} \mathrm{~S}$. The final anisotropic full-matrix least-squares refinement on $\mathrm{F}^{2}$ with 255 variables converged at $R 1=3.31 \%$, for the observed data and wR2 $=9.40 \%$ for all data. The goodness-of-fit was 0.959 . The largest peak in the final difference electron density synthesis was $0.360 \mathrm{e} / / \mathrm{A}^{3}$ and the largest hole was $-0.241 \mathrm{e}^{\prime} / \mathrm{A}^{3}$ with an $\mathrm{RMS}$ deviation of $0.045 \mathrm{e}^{-1} / \mathbb{A}^{3}$. On the basis of the final model the calculated density was $1.229 \mathrm{~g} / \mathrm{cm}^{3}$ and $F(000)$, $3344 \mathrm{e}$. 
Table 2. Sample and crystal data for vdthB.

\begin{tabular}{|c|c|c|}
\hline Identification code & vdthB & \\
\hline Chemical formula & $\mathrm{C}_{18} \mathrm{H}_{28} \mathrm{BN}_{4} \mathrm{O}_{3} \mathrm{~S}$ & \\
\hline Formula weight & $391.31 \mathrm{~g} / \mathrm{mol}$ & \\
\hline Temperature & $100(2) \mathrm{K}$ & \\
\hline Wavelength & $0.71073 \AA$ & \\
\hline $\begin{array}{l}\text { Crystal size } \\
\text { Crystal habit }\end{array}$ & $\begin{array}{l}0.163 \times 0.288 \times 0.381 \mathrm{~mm} \\
\text { clear red block }\end{array}$ & \\
\hline Crystal system & tetragonal & \\
\hline Space group & | 41/a & \\
\hline Unit cell dimensions & $\begin{array}{l}a=27.5302(14) A \\
b=27.5302(14) A \\
c=11.1577(7) \AA\end{array}$ & $\begin{array}{l}\alpha=90^{\circ} \\
\beta=90^{\circ} \\
\gamma=90^{\circ}\end{array}$ \\
\hline Volume & $8456.6(10) A^{3}$ & \\
\hline z & 16 & \\
\hline Density (calculated) & $1.229 \mathrm{~g} / \mathrm{cm}^{3}$ & \\
\hline Absorption coefficient & $0.178 \mathrm{~mm}^{-1}$ & \\
\hline$F(000)$ & 3344 & \\
\hline
\end{tabular}

Table 3. Data collection and structure refinement for vdthB.

\begin{tabular}{|c|c|}
\hline Diffractometer & Bruker D8 QUEST fixed- $\chi$ goniometer \\
\hline Radiation source & $\begin{array}{l}\text { Siemens KFFMO2K-90C sealed tube (Mo } \mathrm{Ka}, \lambda= \\
0.71073 \mathrm{~A})\end{array}$ \\
\hline Theta range for data collection & 1.97 to $33.14^{\circ}$ \\
\hline Index ranges & $-41<=h<=42,-42<=\mathrm{k}<=42,-17<=\mathrm{l}<=17$ \\
\hline Reflections collected & 87798 \\
\hline Independent reflections & $8069[R$ (int) $=0.0478]$ \\
\hline $\begin{array}{l}\text { Coverage of independent } \\
\text { reflections }\end{array}$ & $100.0 \%$ \\
\hline Absorption correction & Multi-Scan \\
\hline $\begin{array}{l}\text { Max. and min. transmission } \\
\text { Structure solution technique }\end{array}$ & $\begin{array}{l}0.7465 \text { and } 0.7013 \\
\text { direct methods }\end{array}$ \\
\hline Structure solution program & XT, VERSION 2014/5 \\
\hline Refinement method & Full-matrix least-squares on $\mathrm{F}^{2}$ \\
\hline Refinement program & SHELXL-2017/1 (Sheldrick, 2017) \\
\hline Function minimized & $\Sigma w\left(F_{0}^{2}-F_{c}^{2}\right)^{2}$ \\
\hline Data / restraints / parameters & $8069 / 0 / 255$ \\
\hline Goodness-of-fit on $F^{2}$ & 0.959 \\
\hline$\Delta / \sigma_{\max }$ & 0.003 \\
\hline \multirow[t]{2}{*}{ Final $R$ indices } & 6504 data; $\mid>2 \sigma(l) \quad R 1=0.0331, w R 2=0.0842$ \\
\hline & all data $\quad R 1=0.0463$, wR2 $=0.0940$ \\
\hline Weighting scheme & $\begin{array}{l}\mathrm{w}=1 /\left[\sigma^{2}\left(\mathrm{~F}_{0}^{2}\right)+(0.0488 \mathrm{P})^{2}+4.3962 \mathrm{P}\right] \\
\text { where } \mathrm{P}=\left(\mathrm{Fo}_{0}^{2}+2 \mathrm{~F}_{\mathrm{c}}^{2}\right) / 3\end{array}$ \\
\hline Largest diff. peak and hole & 0.360 and $-0.241 e \mathbb{A}^{-3}$ \\
\hline R.M.S. deviation from mean & $0.045 \mathrm{eA}^{-3}$ \\
\hline
\end{tabular}


Table 4. Atomic coordinates and equivalent isotropic atomic

\section{displacement parameters $\left(\AA^{2}\right)$ for vdthB.}

$U(e q)$ is defined as one third of the trace of the orthogonalized $U_{;}$ tensor.

\begin{tabular}{llcll}
\hline \multicolumn{1}{c}{$\mathbf{x} / \mathbf{a}$} & \multicolumn{1}{c}{$\mathbf{y} / \mathbf{b}$} & \multicolumn{1}{c}{$\mathbf{z} / \mathbf{c}$} & \multicolumn{1}{c}{$\mathbf{U}(\mathrm{eq})$} \\
S1 & $0.61216(2)$ & $0.56362(2)$ & $0.55865(2)$ & $0.01875(6)$ \\
O1 & $0.67151(2)$ & $0.69619(2)$ & $0.47523(6)$ & $0.02289(13)$ \\
O2 & $0.59726(2)$ & $0.66382(2)$ & $0.42699(6)$ & $0.02380(13)$ \\
O3 & $0.59041(2)$ & $0.34990(2)$ & $0.77422(6)$ & $0.02526(13)$ \\
N1 & $0.66234(3)$ & $0.45644(3)$ & $0.75872(7)$ & $0.02102(13)$ \\
N2 & $0.64687(3)$ & $0.41079(3)$ & $0.78665(7)$ & $0.02246(14)$ \\
N3 & $0.57163(2)$ & $0.42510(3)$ & $0.70090(7)$ & $0.01960(13)$ \\
N4 & $0.58550(3)$ & $0.47046(3)$ & $0.66627(7)$ & $0.01992(13)$ \\
C1 & $0.64899(3)$ & $0.73055(3)$ & $0.39137(8)$ & $0.02171(15)$ \\
C2 & $0.67200(4)$ & $0.72213(4)$ & $0.26945(10)$ & $0.0328(2)$ \\
C3 & $0.65945(5)$ & $0.78145(4)$ & $0.43565(12)$ & $0.0405(3)$ \\
C4 & $0.59434(3)$ & $0.71554(3)$ & $0.39531(8)$ & $0.02079(15)$ \\
C5 & $0.56684(3)$ & $0.72070(3)$ & $0.27858(9)$ & $0.02735(18)$ \\
C6 & $0.56648(4)$ & $0.73967(4)$ & $0.49665(10)$ & $0.0378(2)$ \\
C7 & $0.65173(3)$ & $0.61188(3)$ & $0.56005(7)$ & $0.01928(14)$ \\
C8 & $0.68943(3)$ & $0.60283(3)$ & $0.63804(8)$ & $0.02351(16)$ \\
C9 & $0.68658(3)$ & $0.55699(3)$ & $0.69472(8)$ & $0.02336(16)$ \\
C10 & $0.64614(3)$ & $0.53147(3)$ & $0.65988(7)$ & $0.01812(14)$ \\
C11 & $0.63045(3)$ & $0.48309(3)$ & $0.69774(7)$ & $0.01749(13)$ \\
C12 & $0.60226(3)$ & $0.39193(3)$ & $0.75465(7)$ & $0.01884(14)$ \\
C13 & $0.68213(3)$ & $0.38071(3)$ & $0.85245(10)$ & $0.02781(19)$ \\
C14 & $0.72752(4)$ & $0.37375(5)$ & $0.77961(14)$ & $0.0476(3)$ \\
C15 & $0.69232(5)$ & $0.40366(5)$ & $0.97299(11)$ & $0.0464(3)$ \\
C16 & $0.52226(3)$ & $0.41015(3)$ & $0.66476(8)$ & $0.02247(16)$ \\
C17 & $0.52209(4)$ & $0.39458(4)$ & $0.53438(10)$ & $0.0323(2)$ \\
C18 & $0.48601(3)$ & $0.45047(4)$ & $0.68920(9)$ & $0.02986(19)$ \\
B1 & $0.64049(3)$ & $0.65797(3)$ & $0.48584(8)$ & $0.01952(16)$ \\
\hline & & & &
\end{tabular}

Table 5. Bond lengths $(\AA)$ for vdthB.

\begin{tabular}{llll}
\hline S1-C10 & $1.7129(8)$ & $\mathrm{S} 1-\mathrm{C} 7$ & $1.7182(8)$ \\
$\mathrm{O} 1-\mathrm{B} 1$ & $1.3602(11)$ & $\mathrm{O} 1-\mathrm{C} 1$ & $1.4679(10)$ \\
$\mathrm{O} 2-\mathrm{B} 1$ & $1.3689(11)$ & $\mathrm{O} 2-\mathrm{C} 4$ & $1.4693(10)$ \\
$\mathrm{O} 3-\mathrm{C} 12$ & $1.2218(10)$ & $\mathrm{N} 1-\mathrm{C} 11$ & $1.3310(10)$ \\
$\mathrm{N} 1-\mathrm{N} 2$ & $1.3631(10)$ & $\mathrm{N} 2-\mathrm{C} 12$ & $1.3804(11)$ \\
$\mathrm{N} 2-\mathrm{C} 13$ & $1.4720(11)$ & $\mathrm{N} 3-\mathrm{N} 4$ & $1.3619(9)$ \\
$\mathrm{N} 3-\mathrm{C} 12$ & $1.3801(10)$ & $\mathrm{N} 3-\mathrm{C} 16$ & $1.4761(11)$ \\
$\mathrm{N} 4-\mathrm{C} 11$ & $1.3327(10)$ & $\mathrm{C} 1-\mathrm{C} 3$ & $1.5137(13)$ \\
$\mathrm{C} 1-\mathrm{C} 2$ & $1.5184(14)$ & $\mathrm{C} 1-\mathrm{C} 4$ & $1.5609(12)$ \\
$\mathrm{C} 2-\mathrm{H} 2 \mathrm{~A}$ & 0.98 & $\mathrm{C} 2-\mathrm{H} 2 \mathrm{~B}$ & 0.98 \\
$\mathrm{C} 2-\mathrm{H} 2 \mathrm{C}$ & 0.98 & $\mathrm{C} 3-\mathrm{H} 3 \mathrm{~A}$ & 0.98 \\
$\mathrm{C} 3-\mathrm{H} 3 \mathrm{~B}$ & 0.98 & $\mathrm{C} 3-\mathrm{H} 3 \mathrm{C}$ & 0.98 \\
$\mathrm{C} 4-\mathrm{C5}$ & $1.5131(12)$ & $\mathrm{C} 4-\mathrm{C} 6$ & $1.5194(13)$ \\
$\mathrm{C} 5-\mathrm{H} 5 \mathrm{~A}$ & 0.98 & $\mathrm{C} 5-\mathrm{H} 5 \mathrm{~B}$ & 0.98 \\
$\mathrm{C} 5-\mathrm{H} 5 \mathrm{C}$ & 0.98 & $\mathrm{C} 6-\mathrm{H} 6 \mathrm{~A}$ & 0.98 \\
$\mathrm{C} 6-\mathrm{H} 6 \mathrm{~B}$ & 0.98 & $\mathrm{C} 6-\mathrm{H} 6 \mathrm{C}$ & 0.98 \\
$\mathrm{C} 7-\mathrm{C} 8$ & $1.3771(12)$ & $\mathrm{C} 7-\mathrm{B} 1$ & $1.5465(12)$ \\
$\mathrm{C} 8-\mathrm{C} 9$ & $1.4138(12)$ & $\mathrm{C} 8-\mathrm{H} 8$ & 0.95 \\
$\mathrm{C} 9-\mathrm{C} 10$ & $1.3728(11)$ & $\mathrm{C} 9-\mathrm{H} 9$ & 0.95 \\
$\mathrm{C} 10-\mathrm{C} 11$ & $1.4626(11)$ & $\mathrm{C} 13-\mathrm{C} 14$ & $1.5031(16)$ \\
$\mathrm{C} 13-\mathrm{C} 15$ & $1.5122(17)$ & $\mathrm{C} 13-\mathrm{H} 13$ & 1.0
\end{tabular}




\begin{tabular}{llll}
$\mathrm{C} 14-\mathrm{H} 14 \mathrm{~A}$ & 0.98 & $\mathrm{C} 14-\mathrm{H} 14 \mathrm{~B}$ & 0.98 \\
$\mathrm{C} 14-\mathrm{H} 14 \mathrm{C}$ & 0.98 & $\mathrm{C} 15-\mathrm{H} 15 \mathrm{~A}$ & 0.98 \\
$\mathrm{C} 15-\mathrm{H} 15 \mathrm{~B}$ & 0.98 & $\mathrm{C} 15-\mathrm{H} 15 \mathrm{C}$ & 0.98 \\
$\mathrm{C} 16-\mathrm{C} 17$ & $1.5166(14)$ & $\mathrm{C} 16-\mathrm{C} 18$ & $1.5174(13)$ \\
$\mathrm{C} 16-\mathrm{H} 16$ & 1.0 & $\mathrm{C} 17-\mathrm{H} 17 \mathrm{~A}$ & 0.98 \\
$\mathrm{C} 17-\mathrm{H} 17 \mathrm{~B}$ & 0.98 & $\mathrm{C} 17-\mathrm{H} 17 \mathrm{C}$ & 0.98 \\
$\mathrm{C} 18-\mathrm{H} 18 \mathrm{~A}$ & 0.98 & $\mathrm{C} 18-\mathrm{H} 18 \mathrm{~B}$ & 0.98 \\
$\mathrm{C} 18-\mathrm{H} 18 \mathrm{C}$ & 0.98 & & \\
\hline
\end{tabular}

Table 6. Bond angles $\left({ }^{\circ}\right)$ for vdthB.

\begin{tabular}{|c|c|c|c|}
\hline C10-S1-C7 & $92.71(4)$ & $\mathrm{B} 1-\mathrm{O} 1-\mathrm{Cl}$ & $106.78(6)$ \\
\hline $\mathrm{B} 1-\mathrm{O} 2-\mathrm{C} 4$ & $106.08(6)$ & C11-N1-N2 & $114.77(7)$ \\
\hline $\mathrm{N} 1-\mathrm{N} 2-\mathrm{Cl} 2$ & $124.44(7)$ & N1-N2-C13 & $115.26(7)$ \\
\hline $\mathrm{C} 12-\mathrm{N} 2-\mathrm{Cl} 3$ & $120.27(7)$ & N4-N3-C12 & $123.97(7)$ \\
\hline $\mathrm{N} 4-\mathrm{N} 3-\mathrm{Cl} 6$ & $115.87(7)$ & $\mathrm{C} 12-\mathrm{N} 3-\mathrm{Cl} 16$ & $119.79(7)$ \\
\hline C11-N4-N3 & $115.13(7)$ & $\mathrm{O} 1-\mathrm{Cl}-\mathrm{C} 3$ & $107.95(7)$ \\
\hline $\mathrm{O}-\mathrm{Cl}_{1}-\mathrm{C}_{2}$ & $107.25(7)$ & $\mathrm{C} 3-\mathrm{Cl}_{1}-\mathrm{Cl}_{2}$ & $110.75(9)$ \\
\hline $\mathrm{O}-\mathrm{Cl}-\mathrm{C} 4$ & $102.63(6)$ & $\mathrm{C} 3-\mathrm{Cl}-\mathrm{C} 4$ & $114.79(8)$ \\
\hline $\mathrm{C} 2-\mathrm{Cl}-\mathrm{C} 4$ & $112.77(8)$ & $\mathrm{C} 1-\mathrm{C} 2-\mathrm{H} 2 \mathrm{~A}$ & 109.5 \\
\hline $\mathrm{C} 1-\mathrm{C} 2-\mathrm{H} 2 \mathrm{~B}$ & 109.5 & $\mathrm{H} 2 \mathrm{~A}-\mathrm{C} 2-\mathrm{H} 2 \mathrm{~B}$ & 109.5 \\
\hline $\mathrm{C} 1-\mathrm{C}_{2}-\mathrm{H} 2 \mathrm{C}$ & 109.5 & $\mathrm{H} 2 \mathrm{~A}-\mathrm{C} 2-\mathrm{H} 2 \mathrm{C}$ & 109.5 \\
\hline $\mathrm{H} 2 \mathrm{~B}-\mathrm{C} 2-\mathrm{H} 2 \mathrm{C}$ & 109.5 & $\mathrm{C} 1-\mathrm{C} 3-\mathrm{H} 3 \mathrm{~A}$ & 109.5 \\
\hline $\mathrm{Cl}-\mathrm{C} 3-\mathrm{H} 3 \mathrm{~B}$ & 109.5 & $\mathrm{H} 3 \mathrm{~A}-\mathrm{C} 3-\mathrm{H} 3 \mathrm{~B}$ & 109.5 \\
\hline $\mathrm{Cl}-\mathrm{C} 3-\mathrm{H} 3 \mathrm{C}$ & 109.5 & $\mathrm{H} 3 \mathrm{~A}-\mathrm{C} 3-\mathrm{H} 3 \mathrm{C}$ & 109.5 \\
\hline $\mathrm{H} 3 \mathrm{~B}-\mathrm{C} 3-\mathrm{H} 3 \mathrm{C}$ & 109.5 & $\mathrm{O} 2-\mathrm{C} 4-\mathrm{C} 5$ & $109.00(7)$ \\
\hline $\mathrm{O} 2-\mathrm{C} 4-\mathrm{C} 6$ & $105.81(7)$ & $\mathrm{C} 5-\mathrm{C} 4-\mathrm{C} 6$ & $110.30(8)$ \\
\hline $\mathrm{O} 2-\mathrm{C} 4-\mathrm{Cl}$ & $102.16(6)$ & $\mathrm{C} 5-\mathrm{C} 4-\mathrm{Cl}$ & $115.67(7)$ \\
\hline $\mathrm{C} 6-\mathrm{C} 4-\mathrm{Cl}$ & $113.06(8)$ & $\mathrm{C} 4-\mathrm{C} 5-\mathrm{H} 5 \mathrm{~A}$ & 109.5 \\
\hline $\mathrm{C} 4-\mathrm{C} 5-\mathrm{H} 5 \mathrm{~B}$ & 109.5 & $\mathrm{H} 5 \mathrm{~A}-\mathrm{C} 5-\mathrm{H} 5 \mathrm{~B}$ & 109.5 \\
\hline $\mathrm{C} 4-\mathrm{C} 5-\mathrm{H} 5 \mathrm{C}$ & 109.5 & $\mathrm{H} 5 \mathrm{~A}-\mathrm{C} 5-\mathrm{H} 5 \mathrm{C}$ & 109.5 \\
\hline $\mathrm{H} 5 \mathrm{~B}-\mathrm{C} 5-\mathrm{H} 5 \mathrm{C}$ & 109.5 & $\mathrm{C} 4-\mathrm{C} 6-\mathrm{H} 6 \mathrm{~A}$ & 109.5 \\
\hline $\mathrm{C} 4-\mathrm{C} 6-\mathrm{H} 6 \mathrm{~B}$ & 109.5 & $\mathrm{H} 6 \mathrm{~A}-\mathrm{C} 6-\mathrm{H} 6 \mathrm{~B}$ & 109.5 \\
\hline $\mathrm{C} 4-\mathrm{C} 6-\mathrm{H} 6 \mathrm{C}$ & 109.5 & $\mathrm{H} 6 \mathrm{~A}-\mathrm{C} 6-\mathrm{H} 6 \mathrm{C}$ & 109.5 \\
\hline $\mathrm{H} 6 \mathrm{~B}-\mathrm{C} 6-\mathrm{H} 6 \mathrm{C}$ & 109.5 & $\mathrm{C} 8-\mathrm{C} 7-\mathrm{B} 1$ & $129.60(8)$ \\
\hline$C 8-C 7-51$ & $110.11(6)$ & $B 1-C 7-51$ & $120.18(6)$ \\
\hline $\mathrm{C} 7-\mathrm{CB}-\mathrm{Cg}$ & $113.72(7)$ & $\mathrm{C} 7-\mathrm{C} 8-\mathrm{H} 8$ & 123.1 \\
\hline $\mathrm{C}-\mathrm{C} 8-\mathrm{H} 8$ & 123.1 & $\mathrm{C} 10-\mathrm{Cg}-\mathrm{C} 8$ & $112.04(7)$ \\
\hline $\mathrm{ClO}-\mathrm{Cg}-\mathrm{H} 9$ & 124.0 & $\mathrm{C} 8-\mathrm{Cg}-\mathrm{H} 9$ & 124.0 \\
\hline $\mathrm{C} 9-\mathrm{Cl}-\mathrm{Cl1}$ & $128.60(7)$ & $\mathrm{Cg}-\mathrm{C} 10-\mathrm{SI}$ & $111.42(6)$ \\
\hline C11-C10-S1 & $119.98(6)$ & N1-C11-N4 & $127.12(7)$ \\
\hline $\mathrm{N} 1-\mathrm{Cl}-\mathrm{Cl}$ & $117.06(7)$ & $\mathrm{N} 4-\mathrm{Cl}-\mathrm{C} 10$ & $115.82(7)$ \\
\hline $\mathrm{O} 3-\mathrm{C} 12-\mathrm{N} 3$ & $122.76(8)$ & $\mathrm{O} 3-\mathrm{C} 12-\mathrm{N} 2$ & $123.19(8)$ \\
\hline $\mathrm{N} 3-\mathrm{C} 12-\mathrm{N} 2$ & $114.03(7)$ & N2-C13-C14 & $110.51(9)$ \\
\hline $\mathrm{N} 2-\mathrm{C} 13-\mathrm{Cl} 15$ & $109.32(8)$ & $\mathrm{C} 14-\mathrm{C}_{13}-\mathrm{C}_{15}$ & $112.33(10)$ \\
\hline $\mathrm{N} 2-\mathrm{C} 13-\mathrm{H} 13$ & 108.2 & $\mathrm{C} 14-\mathrm{C} 13-\mathrm{H} 13$ & 108.2 \\
\hline $\mathrm{C} 15-\mathrm{C} 13-\mathrm{H} 13$ & 108.2 & $\mathrm{C} 13-\mathrm{Cl} 14-\mathrm{H} 14 \mathrm{~A}$ & 109.5 \\
\hline $\mathrm{C} 13-\mathrm{C} 14-\mathrm{H} 14 \mathrm{~B}$ & 109.5 & $\mathrm{H} 14 \mathrm{~A}-\mathrm{Cl} 14-\mathrm{H} 14 \mathrm{~B}$ & 109.5 \\
\hline $\mathrm{C} 13-\mathrm{Cl} 14-\mathrm{H} 14 \mathrm{C}$ & 109.5 & $\mathrm{H} 14 \mathrm{~A}-\mathrm{Cl} 14-\mathrm{H} 14 \mathrm{C}$ & 109.5 \\
\hline $\mathrm{H} 14 \mathrm{~B}-\mathrm{C} 14-\mathrm{H} 14 \mathrm{C}$ & 109.5 & $\mathrm{C} 13-\mathrm{C} 15-\mathrm{H} 15 \mathrm{~A}$ & 109.5 \\
\hline $\mathrm{C} 13-\mathrm{C} 15-\mathrm{H} 15 \mathrm{~B}$ & 109.5 & $\mathrm{H} 15 \mathrm{~A}-\mathrm{C} 15-\mathrm{H} 15 \mathrm{~B}$ & 109.5 \\
\hline $\mathrm{C} 13-\mathrm{C} 15-\mathrm{H} 15 \mathrm{C}$ & 109.5 & $\mathrm{H} 15 \mathrm{~A}-\mathrm{C} 15-\mathrm{H} 15 \mathrm{C}$ & 109.5 \\
\hline $\mathrm{H} 15 \mathrm{~B}-\mathrm{C} 15-\mathrm{H} 15 \mathrm{C}$ & 109.5 & $\mathrm{~N} 3-\mathrm{Cl}-\mathrm{Cl}$ & $110.10(7)$ \\
\hline N3-C16-C18 & $110.65(7)$ & $\mathrm{C} 17-\mathrm{C} 16-\mathrm{C} 18$ & $112.16(8)$ \\
\hline $\mathrm{N} 3-\mathrm{Cl} 16-\mathrm{H} 16$ & 107.9 & $\mathrm{C} 17-\mathrm{Cl} 16-\mathrm{H} 16$ & 107.9 \\
\hline $\mathrm{C} 18-\mathrm{C} 16-\mathrm{H} 16$ & 107.9 & $\mathrm{C} 16-\mathrm{C} 17-\mathrm{H} 17 \mathrm{~A}$ & 109.5 \\
\hline $\mathrm{C} 16-\mathrm{C} 17-\mathrm{H} 17 \mathrm{~B}$ & 109.5 & $\mathrm{H} 17 \mathrm{~A}-\mathrm{C} 17-\mathrm{H} 17 \mathrm{~B}$ & 109.5 \\
\hline
\end{tabular}




\begin{tabular}{llll}
$\mathrm{C} 16-\mathrm{C} 17-\mathrm{H} 17 \mathrm{C}$ & 109.5 & $\mathrm{H} 17 \mathrm{~A}-\mathrm{C} 17-\mathrm{H} 17 \mathrm{C}$ & 109.5 \\
$\mathrm{H} 17 \mathrm{~B}-\mathrm{C} 17-\mathrm{H} 17 \mathrm{C}$ & 109.5 & $\mathrm{C} 16-\mathrm{C} 18-\mathrm{H} 18 \mathrm{~A}$ & 109.5 \\
$\mathrm{C} 16-\mathrm{C} 18-\mathrm{H} 18 \mathrm{~B}$ & 109.5 & $\mathrm{H} 18 \mathrm{~A}-\mathrm{C} 18-\mathrm{H} 18 \mathrm{~B}$ & 109.5 \\
$\mathrm{C} 16-\mathrm{C} 18-\mathrm{H} 18 \mathrm{C}$ & 109.5 & $\mathrm{H} 18 \mathrm{~A}-\mathrm{C} 18-\mathrm{H} 18 \mathrm{C}$ & 109.5 \\
$\mathrm{H} 18 \mathrm{~B}-\mathrm{C} 18-\mathrm{H} 18 \mathrm{C}$ & 109.5 & $01-\mathrm{B} 1-\mathrm{O} 2$ & $114.41(7)$ \\
$\mathrm{O} 1-\mathrm{B} 1-\mathrm{C} 7$ & $123.76(8)$ & $\mathrm{O} 2-\mathrm{B} 1-\mathrm{C} 7$ & $121.82(8)$ \\
\hline
\end{tabular}

Table 7. Torsion angles $\left({ }^{\circ}\right)$ for vdthB.

\begin{tabular}{|c|c|c|c|}
\hline $\bar{C} 11-\mathrm{N} 1-\mathrm{N} 2-\mathrm{C} 12$ & $0.87(12)$ & C11-N1-N2-C13 & $179.07(8)$ \\
\hline C12-N3-N4-C11 & $-5.95(12)$ & C16-N3-N4-C11 & $-178.87(7)$ \\
\hline $\mathrm{B} 1-\mathrm{O} 1-\mathrm{Cl}-\mathrm{C} 3$ & $142.05(9)$ & $\mathrm{B} 1-\mathrm{O} 1-\mathrm{Cl}-\mathrm{C} 2$ & $-98.58(8)$ \\
\hline $\mathrm{B} 1-\mathrm{O} 1-\mathrm{Cl}-\mathrm{C} 4$ & $20.43(9)$ & $\mathrm{B} 1-\mathrm{O} 2-\mathrm{C} 4-\mathrm{C} 5$ & $147.81(7)$ \\
\hline $\mathrm{B} 1-\mathrm{O} 2-\mathrm{C} 4-\mathrm{C} 6$ & $-93.59(9)$ & $\mathrm{B} 1-\mathrm{O} 2-\mathrm{C} 4-\mathrm{Cl}$ & $24.93(8)$ \\
\hline $\mathrm{O} 1-\mathrm{C} 1-\mathrm{C} 4-\mathrm{O} 2$ & $-27.39(8)$ & $\mathrm{C} 3-\mathrm{Cl}_{1}-\mathrm{C} 4-\mathrm{O} 2$ & $-144.22(8)$ \\
\hline $\mathrm{C} 2-\mathrm{Cl}_{1}-\mathrm{C} 4-\mathrm{O} 2$ & $87.67(8)$ & $\mathrm{O} 1-\mathrm{Cl}-\mathrm{C} 4-\mathrm{C} 5$ & $-145.62(7)$ \\
\hline $\mathrm{C} 3-\mathrm{Cl}-\mathrm{C} 4-\mathrm{C} 5$ & $97.55(10)$ & $\mathrm{C} 2-\mathrm{C} 1-\mathrm{C} 4-\mathrm{C} 5$ & $-30.56(10)$ \\
\hline $\mathrm{O}-\mathrm{Cl}-\mathrm{C} 4-\mathrm{C} 6$ & $85.85(8)$ & $\mathrm{C} 3-\mathrm{Cl}-\mathrm{C} 4-\mathrm{C} 6$ & $-30.98(11)$ \\
\hline $\mathrm{C} 2-\mathrm{Cl}-\mathrm{C} 4-\mathrm{C} 6$ & $-159.09(8)$ & $\mathrm{C} 10-\mathrm{S} 1-\mathrm{C} 7-\mathrm{C} 8$ & $-0.70(7)$ \\
\hline $\mathrm{C} 10-S 1-C 7-B 1$ & $-177.31(7)$ & $\mathrm{B} 1-\mathrm{C} 7-\mathrm{C} 8-\mathrm{C} 9$ & $177.03(9)$ \\
\hline $\mathrm{S1-C7-C8-C9}$ & $0.83(10)$ & $\mathrm{C} 7-\mathrm{C} 8-\mathrm{Cg}-\mathrm{Cl}$ & $-0.54(12)$ \\
\hline $\mathrm{C} 8-\mathrm{C} 9-\mathrm{C} 10-\mathrm{Cl1}$ & $179.88(8)$ & C8-C9-C10-S1 & $0.00(10)$ \\
\hline C7-S1-C10-C9 & $0.40(7)$ & C7-S1-C10-C11 & $-179.49(7)$ \\
\hline $\mathrm{N} 2-\mathrm{N} 1-\mathrm{C} 11-\mathrm{N} 4$ & $2.70(13)$ & $\mathrm{N} 2-\mathrm{N} 1-\mathrm{C} 11-\mathrm{C} 10$ & $-176.84(7)$ \\
\hline N3-N4-C11-N1 & $-0.31(13)$ & N3-N4-C11-C10 & $179.24(7)$ \\
\hline $\mathrm{C} 9-\mathrm{Cl} 10-\mathrm{Cl1}-\mathrm{N} 1$ & $-11.81(13)$ & $\mathrm{S} 1-\mathrm{C} 10-\mathrm{C} 11-\mathrm{N} 1$ & $168.06(6)$ \\
\hline $\mathrm{C} 9-\mathrm{Cl}$-Cl1-N4 & $168.60(9)$ & $\mathrm{S} 1-\mathrm{C} 10-\mathrm{C} 11-\mathrm{N} 4$ & $-11.54(10)$ \\
\hline $\mathrm{N} 4-\mathrm{N} 3-\mathrm{C} 12-\mathrm{O} 3$ & $-172.59(8)$ & $\mathrm{C} 16-\mathrm{N} 3-\mathrm{C} 12-\mathrm{O} 3$ & $0.07(12)$ \\
\hline $\mathrm{N} 4-\mathrm{N} 3-\mathrm{C} 12-\mathrm{N} 2$ & $8.84(12)$ & $\mathrm{C} 16-\mathrm{N} 3-\mathrm{C} 12-\mathrm{N} 2$ & $-178.50(7)$ \\
\hline $\mathrm{N} 1-\mathrm{N} 2-\mathrm{C} 12-\mathrm{O} 3$ & $175.27(8)$ & $\mathrm{C} 13-\mathrm{N} 2-\mathrm{C} 12-\mathrm{O} 3$ & $-2.85(13)$ \\
\hline $\mathrm{N} 1-\mathrm{N} 2-\mathrm{C} 12-\mathrm{N} 3$ & $-6.16(12)$ & $\mathrm{C} 13-\mathrm{N} 2-\mathrm{C} 12-\mathrm{N} 3$ & $175.71(8)$ \\
\hline N1-N2-C13-C14 & $-60.64(12)$ & $\mathrm{C} 12-\mathrm{N} 2-\mathrm{C} 13-\mathrm{C} 14$ & $117.65(10)$ \\
\hline N1-N2-C13-C15 & $63.48(11)$ & $\mathrm{C} 12-\mathrm{N} 2-\mathrm{C} 13-\mathrm{C} 15$ & $-118.23(10)$ \\
\hline $\mathrm{N} 4-\mathrm{N} 3-\mathrm{C} 16-\mathrm{C} 17$ & $78.95(10)$ & $\mathrm{C} 12-\mathrm{N} 3-\mathrm{C} 16-\mathrm{C} 17$ & $-94.29(10)$ \\
\hline $\mathrm{N} 4-\mathrm{N} 3-\mathrm{C} 16-\mathrm{C} 18$ & $-45.60(10)$ & $\mathrm{C} 12-\mathrm{N} 3-\mathrm{C} 16-\mathrm{C} 18$ & $141.17(8)$ \\
\hline $\mathrm{C} 1-\mathrm{O} 1-\mathrm{B} 1-\mathrm{O} 2$ & $-5.30(10)$ & $\mathrm{Cl}-\mathrm{O} 1-\mathrm{B} 1-\mathrm{C} 7$ & $176.08(8)$ \\
\hline $\mathrm{C} 4-\mathrm{O} 2-\mathrm{B} 1-\mathrm{O} 1$ & $-13.57(10)$ & $\mathrm{C} 4-\mathrm{O} 2-\mathrm{B} 1-\mathrm{C} 7$ & $165.08(8)$ \\
\hline C8-C7-B1-O1 & $9.09(15)$ & $\mathrm{S} 1-\mathrm{C} 7-\mathrm{B} 1-\mathrm{O} 1$ & $-175.04(7)$ \\
\hline $\mathrm{C} 8-\mathrm{C} 7-\mathrm{B} 1-\mathrm{O} 2$ & $-169.44(9)$ & $\mathrm{S} 1-\mathrm{C} 7-\mathrm{B} 1-\mathrm{O} 2$ & $6.43(12)$ \\
\hline
\end{tabular}

Table 8. Anisotropic atomic displacement parameters $\left(\AA^{2}\right)$ for vdthB.

The anisotropic atomic displacement factor exponent takes the form: $-2 \pi^{2}\left[\mathrm{~h}^{2} \mathrm{a}^{2} \mathrm{U}_{11}+\ldots+2 \mathrm{~h} \mathrm{k} \mathrm{a}^{*} \mathrm{~b}^{*}\right.$ $\left.\mathrm{U}_{12}\right]$

\begin{tabular}{lllllll}
\hline & \multicolumn{1}{c}{$\mathbf{U}_{11}$} & \multicolumn{1}{c}{$\mathbf{U}_{22}$} & \multicolumn{1}{c}{$\mathbf{U}_{33}$} & \multicolumn{1}{c}{$\mathbf{U}_{23}$} & \multicolumn{1}{c}{$\mathbf{U}_{13}$} & \multicolumn{1}{c}{$\mathbf{U}_{12}$} \\
S1 & $0.01745(9)$ & $0.01839(9)$ & $0.02041(10)$ & $0.00377(7)$ & $-0.00246(6)$ & $0.00065(6)$ \\
O1 & $0.0238(3)$ & $0.0185(3)$ & $0.0263(3)$ & $0.0055(2)$ & $-0.0063(2)$ & $-0.0004(2)$ \\
O2 & $0.0203(3)$ & $0.0193(3)$ & $0.0318(3)$ & $0.0069(2)$ & $-0.0023(2)$ & $0.0006(2)$ \\
O3 & $0.0256(3)$ & $0.0171(3)$ & $0.0331(3)$ & $0.0035(2)$ & $0.0031(3)$ & $-0.0017(2)$ \\
N1 & $0.0193(3)$ & $0.0176(3)$ & $0.0262(3)$ & $0.0049(3)$ & $-0.0041(3)$ & $-0.0010(2)$ \\
N2 & $0.0193(3)$ & $0.0181(3)$ & $0.0300(4)$ & $0.0071(3)$ & $-0.0038(3)$ & $0.0006(2)$ \\
N3 & $0.0172(3)$ & $0.0190(3)$ & $0.0226(3)$ & $0.0029(2)$ & $-0.0013(2)$ & $-0.0015(2)$ \\
N4 & $0.0191(3)$ & $0.0181(3)$ & $0.0225(3)$ & $0.0043(2)$ & $-0.0027(2)$ & $-0.0004(2)$ \\
C1 & $0.0239(4)$ & $0.0169(3)$ & $0.0244(4)$ & $0.0048(3)$ & $-0.0050(3)$ & $-0.0005(3)$ \\
C2 & $0.0271(4)$ & $0.0409(5)$ & $0.0302(5)$ & $0.0134(4)$ & $0.0042(4)$ & $0.0022(4)$
\end{tabular}




\begin{tabular}{|c|c|c|c|c|c|c|}
\hline & $\mathbf{U}_{11}$ & $U_{22}$ & $\mathbf{U}_{33}$ & $\mathbf{U}_{23}$ & $U_{13}$ & $U_{12}$ \\
\hline C3 & $0.0519(7)$ & $0.0181(4)$ & $0.0515(7)$ & $0.0036(4)$ & $-0.0223(5)$ & $-0.0047(4)$ \\
\hline C4 & $0.0217(3)$ & $0.0180(3)$ & $0.0227(4)$ & $0.0019(3)$ & $-0.0009(3)$ & $0.0049(3)$ \\
\hline C5 & $0.0264(4)$ & $0.0261(4)$ & $0.0295(4)$ & $0.0028(3)$ & $-0.0073(3)$ & $0.0042(3)$ \\
\hline C6 & $0.0405(6)$ & $0.0419(6)$ & $0.0309(5)$ & $0.0005(4)$ & $0.0051(4)$ & $0.0204(5)$ \\
\hline$C 7$ & $0.0194(3)$ & $0.0176(3)$ & $0.0209(3)$ & $0.0017(3)$ & $-0.0005(3)$ & $0.0014(3)$ \\
\hline $\mathrm{CB}$ & $0.0228(4)$ & $0.0187(3)$ & $0.0291(4)$ & $0.0039(3)$ & $-0.0049(3)$ & $-0.0038(3)$ \\
\hline C9 & $0.0238(4)$ & $0.0203(3)$ & $0.0260(4)$ & $0.0046(3)$ & $-0.0082(3)$ & $-0.0009(3)$ \\
\hline $\mathrm{C} 10$ & $0.0194(3)$ & $0.0162(3)$ & $0.0187(3)$ & $0.0022(3)$ & $-0.0018(3)$ & $0.0005(2)$ \\
\hline C11 & $0.0174(3)$ & $0.0173(3)$ & $0.0178(3)$ & $0.0017(3)$ & $-0.0022(3)$ & $0.0013(2)$ \\
\hline $\mathrm{C} 12$ & $0.0189(3)$ & $0.0172(3)$ & $0.0205(3)$ & $0.0016(3)$ & $0.0023(3)$ & $0.0013(3)$ \\
\hline $\mathrm{C} 13$ & $0.0226(4)$ & $0.0223(4)$ & $0.0386(5)$ & $0.0105(4)$ & $-0.0053(3)$ & $0.0023(3)$ \\
\hline C14 & $0.0325(5)$ & $0.0461(7)$ & $0.0644(8)$ & $0.0048(6)$ & $0.0035(5)$ & $0.0185(5)$ \\
\hline C15 & $0.0553(7)$ & $0.0502(7)$ & $0.0339(6)$ & $0.0112(5)$ & $-0.0139(5)$ & $0.0139(6)$ \\
\hline $\mathrm{C} 16$ & $0.0183(3)$ & $0.0262(4)$ & $0.0230(4)$ & $0.0015(3)$ & $-0.0003(3)$ & $-0.0036(3)$ \\
\hline $\mathrm{C} 17$ & $0.0248(4)$ & $0.0418(5)$ & $0.0304(5)$ & $-0.0093(4)$ & $-0.0024(3)$ & $-0.0036(4)$ \\
\hline $\mathrm{C} 18$ & $0.0190(4)$ & $0.0406(5)$ & $0.0300(4)$ & $-0.0024(4)$ & $-0.0004(3)$ & $0.0025(3)$ \\
\hline B1 & $0.0202(4)$ & $0.0179(4)$ & $0.0205(4)$ & $0.0016(3)$ & $0.0011(3)$ & $0.0020(3)$ \\
\hline
\end{tabular}

Table 9. Hydrogen atomic coordinates and isotropic atomic displacement parameters $\left(\AA^{2}\right)$ for vdthB.

\begin{tabular}{|c|c|c|c|c|}
\hline & $x / a$ & $y / b$ & $z / c$ & $\mathbf{u}(\mathrm{eq})$ \\
\hline $\mathrm{H} 2 \mathrm{~A}$ & 0.6652 & 0.6889 & 0.2429 & 0.049 \\
\hline $\mathrm{H} 2 \mathrm{~B}$ & 0.7072 & 0.7269 & 0.2752 & 0.049 \\
\hline $\mathrm{H} 2 \mathrm{C}$ & 0.6584 & 0.7452 & 0.2115 & 0.049 \\
\hline $\mathrm{H} 3 \mathrm{~A}$ & 0.6945 & 0.7874 & 0.4331 & 0.061 \\
\hline $\mathrm{H} 3 \mathrm{~B}$ & 0.6478 & 0.7849 & 0.5182 & 0.061 \\
\hline $\mathrm{H} 3 \mathrm{C}$ & 0.6428 & 0.8050 & 0.3843 & 0.061 \\
\hline $\mathrm{H} 5 \mathrm{~A}$ & 0.5818 & 0.7000 & 0.2175 & 0.041 \\
\hline $\mathrm{H} 5 \mathrm{~B}$ & 0.5679 & 0.7546 & 0.2521 & 0.041 \\
\hline $\mathrm{H} 5 \mathrm{C}$ & 0.5330 & 0.7109 & 0.2905 & 0.041 \\
\hline $\mathrm{H} 6 \mathrm{~A}$ & 0.5616 & 0.7741 & 0.4779 & 0.057 \\
\hline $\mathrm{H} 6 \mathrm{~B}$ & 0.5850 & 0.7367 & 0.5713 & 0.057 \\
\hline $\mathrm{H} 6 \mathrm{C}$ & 0.5349 & 0.7238 & 0.5062 & 0.057 \\
\hline H8 & 0.7150 & 0.6253 & 0.6524 & 0.028 \\
\hline $\mathrm{H9}$ & 0.7099 & 0.5452 & 0.7504 & 0.028 \\
\hline $\mathrm{H} 13$ & 0.6672 & 0.3481 & 0.8665 & 0.033 \\
\hline $\mathrm{H} 14 \mathrm{~A}$ & 0.7193 & 0.3584 & 0.7030 & 0.071 \\
\hline $\mathrm{H} 14 \mathrm{~B}$ & 0.7502 & 0.3529 & 0.8236 & 0.071 \\
\hline $\mathrm{H} 14 \mathrm{C}$ & 0.7427 & 0.4054 & 0.7647 & 0.071 \\
\hline $\mathrm{H} 15 \mathrm{~A}$ & 0.7078 & 0.4353 & 0.9612 & 0.07 \\
\hline $\mathrm{H} 15 \mathrm{~B}$ & 0.7140 & 0.3825 & 1.0192 & 0.07 \\
\hline $\mathrm{H} 15 \mathrm{C}$ & 0.6617 & 0.4080 & 1.0167 & 0.07 \\
\hline $\mathrm{H} 16$ & 0.5128 & 0.3814 & 0.7145 & 0.027 \\
\hline H17A & 0.4899 & 0.3820 & 0.5133 & 0.048 \\
\hline $\mathrm{H} 17 \mathrm{~B}$ & 0.5465 & 0.3692 & 0.5222 & 0.048 \\
\hline $\mathrm{H} 17 \mathrm{C}$ & 0.5297 & 0.4226 & 0.4834 & 0.048 \\
\hline $\mathrm{H} 18 \mathrm{~A}$ & 0.4929 & 0.4781 & 0.6366 & 0.045 \\
\hline $\mathrm{H} 18 \mathrm{~B}$ & 0.4887 & 0.4607 & 0.7730 & 0.045 \\
\hline $\mathrm{H} 18 \mathrm{C}$ & 0.4530 & 0.4386 & 0.6738 & 0.045 \\
\hline
\end{tabular}

